\title{
Probing correlated quantum many-body systems at the single-particle level
}

\author{
Manuel Endres
}

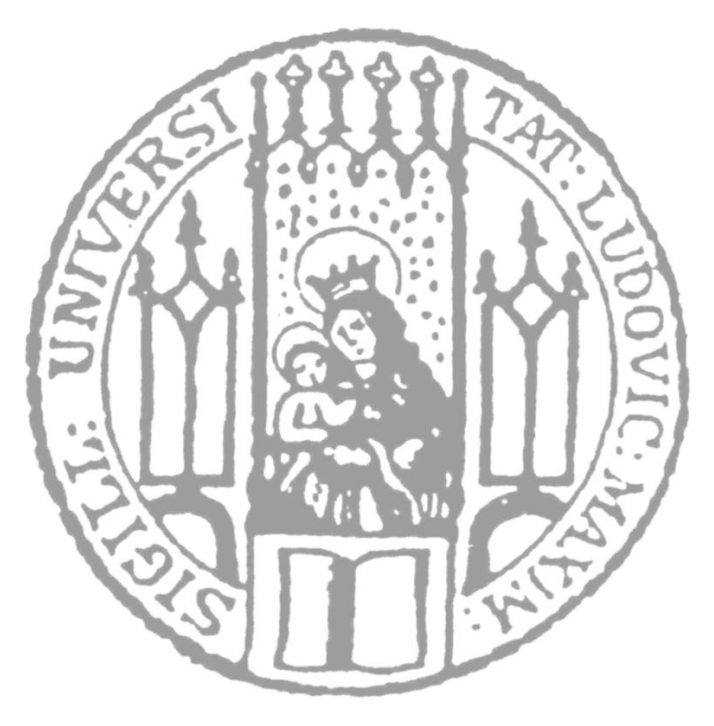

Dissertation der Fakultät für Physik der Ludwig-Maximilians-Universität München 
Erstgutachter: Prof. Dr. Immanuel Bloch Zweitgutachter: Prof. Dr. Stefan Kuhr

Tag der mündlichen Prüfung: 27.2.2013 


\title{
Probing correlated quantum many-body systems at the single-particle level
}

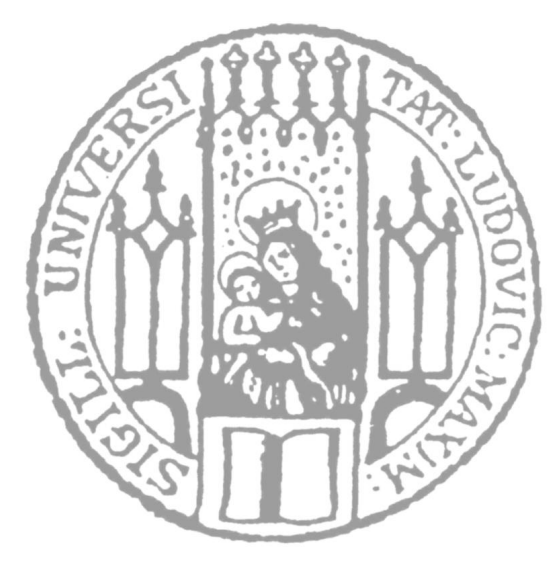

Dissertation der Fakultät für Physik

der Ludwig-Maximilians-Universität München

\author{
vorgelegt von \\ Manuel Endres \\ geboren in Würzburg
}

München, 2013 


\section{Summary}

The detection of correlation and response functions plays a crucial role in the experimental characterization of quantum many-body systems. In this thesis, we present novel techniques for the measurement of such functions at the single-particle level. Specifically, we show the single-atom- and single-site-resolved detection of an ultracold quantum gas in an optical lattice. The quantum gas is described by the Bose-Hubbard model, which features a zero temperature phase transition from a superfluid to a Mott-insulating state, a paradigm example of a quantum phase transition. We used the aforementioned detection techniques to study correlation and response properties across the superfluid-Mott-insulator transition.

The single-atom sensitivity of our method is achieved by fluorescence detection of individual atoms with a high signal-to-noise ratio. A high-resolution objective collects the fluorescence light and yields in situ 'snapshots' of the quantum gas that allow for a single-site-resolved reconstruction of the atomic distribution.

This allowed us to measure two-site and non-local correlation-functions across the superfluid-Mott-insulator transition. Non-local correlation functions are based on the information of an extended region of the system and play an important role for the characterization of low-dimensional quantum phases. While non-local correlation functions were so far only theoretical tools, our results show that they are actually experimentally accessible.

Furthermore, we used a new thermometry scheme, based on the counting of individual thermal excitations, to measure the response of the system to lattice modulation. Using this method, we studied the excitation spectrum of the system across the two-dimensional superfluid-Mott-insulator transition. In particular, we detected a 'Higgs' amplitude mode in the strongly-interacting superfluid close to the transition point where the system is described by an effectively Lorentz-invariant low-energy theory. Our experimental results helped to resolve a debate about the observability of Higgs modes in two-dimensional systems. 


\section{Zusammenfassung}

Korrelations- und Antwortfunktionen sind wichtige Hilfsmittel um QuantenVielteilchensysteme zu charakterisieren. Das Thema dieser Arbeit ist die Messung solcher Funktionen mit Hilfe einer neuartigen Methode, die es erlaubt einzelne Atome in einem Quanten-Vielteilchensystemen nachzuweisen. Diese Technik ermöglicht die Abbildung eines stark korrelierten Quantengases in einem optischen Gitter, wobei einzelne Atome am jeweiligen Gitterplatz detektiert werden. Das Quantengas wird durch das Bose-Hubbard Modell beschrieben, das einen QuantenPhasenübergang von einer suprafluiden zu einer Mott-isolierenden Phase aufzeigt. Der Fokus dieser Arbeit ist die Messung von Korrelations- und Antwortfunktionen in der Nähe des Quanten-Phasenübergangs.

Die neue Technik basiert auf Fluoreszenz-Abbildung einzelner Atome mit hohem Signal-zu-Rauch-Verhältnis. Ein hochauflösendes Objektiv liefert "Schnappschüsse“ des Systems, die es erlauben die Verteilung der Atome im Gitter zu rekonstruieren.

Mit Hilfe der rekonstruierten Verteilung konnten Korrelationen zwischen verschiedenen Gitterplätzen detektiert werden, was auch sogenannte nichtlokale Korrelationensfunktionen einschließt. Letztere basieren auf dem Signal einer ausgedehnten Region und spielen eine wichtige Rolle für die Charakterisierung niedrigdimensionaler Systeme. Während nichtlokale Korrelationen bisher nur ein rein theoretisches Werkzeug darstellten, erlaubt unsere Methode erstmals die experimentelle Detektion.

Des Weiteren wurde ein neuartiges Verfahren entwickelt um die Temperatur des Quantengases zu bestimmen. Es beruht auf der Detektion einzelner thermischer Anregungen des Vielteilchensystems und ist deshalb extrem sensitiv. Durch die hohe Empfindlichkeit ist die Methode besonders für die Messung von Anwortfunktionen geeignet. Hierfür wurde die Tiefe des optischen Gitters mit variabler Frequenz moduliert und die Temperaturerhöhung des Systems aufgezeichnet. Das Verfahren gibt Aufschluss über das Anregungsspektrum des Quantengases. Insbesondere konnte eine „Higgs“-Amplituden-Anregung in der Nähe des Quanten-Phasenübergangs nachgewiesen werden. Die experimentellen Resultate konnten dabei helfen die Diskussion über die Observierbarkeit von Higgs-Anregungen in zweidimensionalen Systemen aufzulösen. 


\section{Contents}

1. Introduction 1

I. Single-site- and single-atom-resolved detection of atomic $\begin{array}{ll}\text { limit Mott insulators } & 7\end{array}$

2. Superfluid-Mott-insulator transition 9

2.1. Bose-Hubbard model . . . . . . . . . . . . . . . . . . . . . 9 9

2.2. Mott-insulating and superfluid states . . . . . . . . . . . . . 11

2.2.1. Mott-insulating state . . . . . . . . . . . . . . . . . . . 11

2.2.2. Superfluid state . . . . . . . . . . . . . . . . . . . 13

2.3. Phase transitions in the Bose-Hubbard model . . . . . . . . . . . . . 15

2.4. Realization of the Bose-Hubbard model in optical lattices . . . . . . . . 17

2.4.1. Optical lattice potential . . . . . . . . . . . . . . . . 17

2.4.2. Derivation of the tunneling and interaction term . . . . . . . . 18

2.4.3. Three-dimensional optical lattice and harmonic confinement . . 21

2.4.4. Discussion . . . . . . . . . . . . . . . . . 24

3. Overview of the experimental procedure 25

3.1. Two-dimensional degenerate gas and vertical lattice . . . . . . . . . . 25

3.2. Horizontal square lattice and adiabatic preparation . . . . . . . . . 26

3.3. Fluorescence imaging of single atoms . . . . . . . . . . . . . . 27

3.4. Parity projection . . . . . . . . . . . . . . . . . . . 28

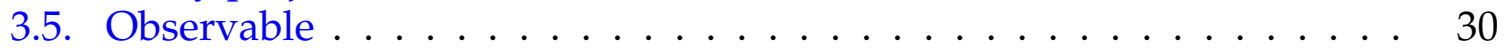

4. Single-site-resolved imaging and thermometry of atomic limit Mott insulators

4.1. Theory for the ground state and the influence of a trapping potential . 33

4.1.1. Eigenspectrum and eigenstates .................. 33

4.1.2. Influence of the trapping potential . . . . . . . . . . . 35

4.2. Single-site-resolved fluorescence images . . . . . . . . . . . . . . . . 36

4.3. Influence of finite temperature and in situ thermometry . . . . . . . . 39

4.4. Summary and conclusion . . . . . . . . . . . . . . . . 43 


\section{Single-site- and single-atom-resolved detection of correla-} tion functions

5. Detection of particle-hole pairs using two-site correlation functions $\quad 47$

5.1. Introduction to particle-hole pairs and two-site correlation functions . $\quad 47$

5.2. Results in one dimension . . . . . . . . . . . . . . . . . . . . . 49

5.3. Results in two dimensions . . . . . . . . . . . . . . . . . . 53

5.4. On-site fluctuations and next-nearest neighbor correlations . . . . . . 54

5.5. Summary and conclusion ..................... 56

6. Non-local correlations in one dimension 57

6.1. Non-local order parameters . . . . . . . . . . . . . . . . . . 57

6.2. Non-local order in the Bose-Hubbard model . . . . . . . . . . . . . 60

6.2.1. Intuitive picture and strong coupling limit . . . . . . . . . . . 60

6.2.2. Analytical results based on Bosonization . . . . . . . . . . . 62

6.2.3. Numerical analysis . . . . . . . . . . . . . . . . . . . 64

6.3. Experimental results for the string order parameter . . . . . . . . . 66

6.4. Multi-site correlations . . . . . . . . . . . . . . . . . 69

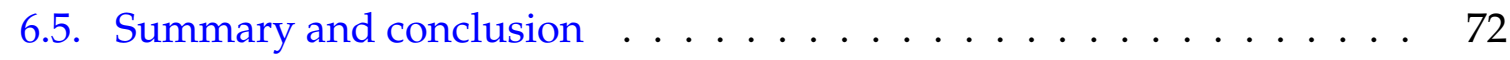

7. Non-local correlations in two dimensions, duality and distribution functions

7.1. Intuitive picture for non-local correlations in the two-dimensional Bose-Hubbard model . . . . . . . . . . . . . . . . . 74

7.2. Non-local correlations and duality . . . . . . . . . . . . . . 76

7.2.1. Quantum Ising model in a transverse field . . . . . . . . . . . 76

7.2.2. Bose-Hubbard model . . . . . . . . . . . . . . . 81

7.3. Distribution functions . . . . . . . . . . . . . . . 86

7.3.1. Atom number distribution . . . . . . . . . . . 86

7.3.2. Parity distribution .................. 89

7.4. Summary and conclusion . . . . . . . . . . . . . 90 
$\begin{array}{ll}\text { III. 'Higgs' amplitude mode } & 91\end{array}$

8. Introduction to amplitude and phase modes 93

8.1. Overview . . . . . . . . . . . . . . . . . 93

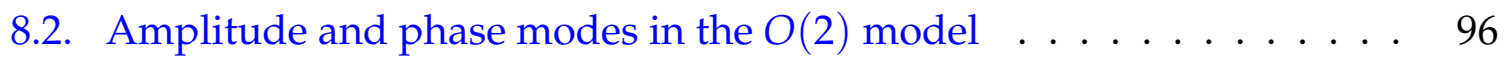

8.2.1. Relation of $\Psi$ to observables . . . . . . . . . . . . . . . . . 97

8.2.2. Relativistic form and free Lagrangian . . . . . . . . . . . . . 98

8.2.3. Amplitude and phase modes . . . . . . . . . . . . . . . 99

8.2.4. Mode softening . . . . . . . . . . . . . . . . . . . . 100

8.3. Anderson-Higgs mechanism . . . . . . . . . . . . . . . . 102

8.4. Comparison with Gross-Pitaevskii dynamics . . . . . . . . . . . . . . 103

8.5. Influence of deviation from integer filling . . . . . . . . . . . . . . 106

8.6. Response functions and interactions between modes . . . . . . . . . . 108

8.6.1. Linear response theory for the Bose-Hubbard model . . . . . . 108

8.6.2. Visibility of the Higgs mode in the two-dimensional $O(2)$ model 111

9. Detection of the Higgs amplitude mode at the $2 \mathrm{~d}$ SF-Mott-insulator transition

9.1. Experimental procedure . . . . . . . . . . . . . . . . 115

9.2. Softening of the mode gap . . . . . . . . . . . . . . . 116

9.3. Raw data and linear response . . . . . . . . . . . . . . . . . . . . 119

9.4. Analysis of the response . . . . . . . . . . . . . . . . 120

9.4.1. Gutzwiller analysis . . . . . . . . . . . . . . . . . . . . 120

9.4.2. Scaling of the low-frequency response . . . . . . . . . . . . . 122

9.4.3. Intermediate frequencies and heuristic model . . . . . . . . . 123

9.4.4. High frequencies and $2 \times 2$ cluster mean field simulation . . . . 124

9.5. Vanishing of the response in the weakly interacting limit . . . . . . . 126

9.6. Softening with respect to J . . . . . . . . . . . . . . . 128

9.7. Discussion of finite-temperature effects . . . . . . . . . . . . . 128

9.8. Gutzwiller calculation and $2 \times 2$ cluster wave functions . . . . . . . . . 129

9.9. Distinguishing phase and amplitude modes . . . . . . . . . . . . . . 130

9.10. Summary and conclusion . . . . . . . . . . . . 135

$\begin{array}{ll}\text { 10. Outlook } & 137\end{array}$ 
A. Experimental details 143

A.1. Preparation of the two-dimensional quantum gas . . . . . . . . 143

A.1.1. Overview . . . . . . . . . . . . . . . . . . . 143

A.1.2. MOT and magnetic evaporation . . . . . . . . . . . . . 144

A.1.3. Transport and loading to vertical lattice . . . . . . . . . . . . 146

A.1.4. Preparation of a single $2 \mathrm{~d}$ system and final evaporation . . . . . 147

A.2. Optical lattices and high-resolution imaging . . . . . . . . . . . 151

B. Numerical methods for correlation functions 155

C. Calculation for non-local correlations in one dimension 159

D. Calculation for non-local correlations in two dimensions 165

$\begin{array}{ll}\text { Bibliography } & 169\end{array}$ 


\section{Introduction}

The use of ultracold atoms for the study of strongly interacting many-body systems has undergone remarkable development in recent years [1]. Prominent examples include reaching the strongly interacting regime of bosonic and fermionic gases in optical lattices [2-6] and studies of the BEC-BCS crossover by means of Feshbach resonances [7-9].

The success of this approach is based on the high degree of control that has been achieved over experimental parameters. In particular, the underlying Hamiltonian is usually known and its parameters, such as the interaction strength or the effective mass, can be accurately determined and tuned over a large range.

Furthermore, cold gases can be probed with versatile detection techniques, such as in situ absorption, in situ phase-contrast, and time-of-flight imaging [3, 10-12]. The latter has been combined with spectroscopic techniques, such as momentum-resolved radio-frequency spectroscopy and momentum-resolved Bragg spectroscopy [13, 14], to gain access to the excitation spectrum of the interacting many-body system.

Recent advances in high-resolution in situ fluorescence-imaging of atoms in optical lattices, demonstrated in Refs. [15, 16] and by our group [17], have pushed these detection capabilities to the fundamental level of individual atoms. Specifically, this technique allows for the single-site-resolved detection of a lattice gas in the Bose-Hubbard regime [18, 19]. The Bose-Hubbard model describes hopping between nearest-neighbor sites and pairwise interactions of particles on the same site. The competition of these two processes gives rise to a quantum phase transition between a superfluid and a Mott-insulating phase [18-20]. The new high-resolution fluorescence detection allows to take 'snapshots' of such an interacting many-body system that directly show the fluctuations with single-particle sensitivity. An introduction to this topic forms the first part of this thesis.

In recent years, other high-resolution imaging techniques have also been successfully used to study ultracold gases in optical lattices, such as scanning electron microscopy [21, 22] and high-resolution in situ absorption imaging [11, 23, 24]. In contrast to high-resolution in situ fluorescence-imaging, these techniques do not have single-atom sensitivity in a single experimental image.

High-resolution fluorescence experiments are able to detect the on-site parity of the number of particles on a lattice site. The average on-site parity has been used to study the superfluid-Mott insulator transition [16] and to develop an in situ temperature measurement in the deep Mott insulating limit [17]. Furthermore, an antiferromagnetic quantum Ising spin chain has been simulated in a tilted optical 
lattice, where nearest-neighbor spin correlations map onto the average on-site parity [25], and an orbital excitation blockade could be directly observed [26].

The high-resolution technique can also be used in reverse to control individual atoms and to modify Hamiltonian parameters on the level of individual lattice sites. In particular, we experimentally demonstrated that the spin of individual atoms in a Mott insulator can be addressed [27]. We subsequently used this technique to study a single, mobile spin-impurity in a strongly interacting one-dimensional system [28].

One of the most advantageous features of high-resolution fluorescence detection is its ability to resolve single particles in each experimental run. This allows for the direct measurement of correlations between fluctuations in different parts of the system, in addition to the average on-site parity. Notably, this is not limited only to correlations between two lattice sites. Rather, the detection of high-order correlation functions between an arbitrary set of sites became feasible. Such non-local correlation functions play an important role for the characterization of quantum phases in low-dimensional many-body systems. We used these possibilities to detect two-site and non-local correlations across the superfluid-Mott-insulator transition [29], which is the focus of Part II of this thesis.

In a subsequent experiment, we extended our measurement to an out of equilibrium system. Specifically, we imaged the spreading of correlations after a sudden change of the Hamiltonian parameters in the Mott insulating regime [30]. Furthermore, in a recent experiment, we used the same technique to detect correlations between Rydberg atoms forming mesoscopic ordered structures [31] demonstrating that the general approach is not restricted to Hubbard-type physics.

As described above, existing detection techniques have been successfully combined with spectroscopic methods to study the excitation spectrum of strongly-interacting cold gases systems. In a similar way, we combined the aforementioned in situ temperature measurement [17], based on single-atom detection, with lattice modulation spectroscopy. In contrast to earlier measurements with lattice modulation [32-34], we could probe our system in the linear response regime as a result of the high sensitivity of the temperature measurement.

We used this tool to detect a 'Higgs' amplitude mode close to the two-dimensional superfluid-Mott insulator transition (Part III). The appearance of such a mode can be understood from the low-energy description of the dynamics close to the superfluidMott insulator phase boundary. If the boundary is crossed at constant density, the description becomes effectively Lorentz invariant, allowing for the existence of a Higgs mode. The mathematical structure behind this excitation is the same as the one for the long-sought Higgs particle of the standard model [35], if interactions between various excitations of the system are neglected. However, the detectability of a Higgs mode in a two-dimensional system has been a matter of debate [20,36-41]. Recent theoretical results $[41,42]$ and our experiments have, to some degree, settled the 
issue and showed that a Higgs mode in two-dimensions, close to a quantum phase transition, is indeed observable.

The control and detection possibilities for strongly-interacting ultracold gases made it possible to perform quantitative studies, for which a theoretical treatment, even using modern numerical methods, is intrinsically difficult or even impossible. This includes, e.g., the study of out-of-equilibrium dynamics of bosonic systems [43] or the equation of state measurement for fermions at the BEC-BCS crossover (see Refs. [1,9] and references therein). Such experimental results can, in turn, be used to check or falsify theoretical treatments, where the quantitative validity of the latter is not a priori guaranteed. To some degree, our experimental results for the Higgs mode and the recent theoretical treatments [41,42] fall into this category.

One of the general goals for the field of ultracold atoms is to achieve results that directly help to solve outstanding problems in solid-state physics, such as the nature of high- $T_{\mathcal{C}}$ superconductors. In most cases, a direct realization of an ultracold-gases analog of an actual solid-state system might be impossible. However, the comparison of experimental and theoretical results, in the sense of the previous paragraph, is likely to improve our understanding and the theoretical treatment of quantum many-body systems in general. In turn, some of these improvements might find an application in solid-state physics or in other fields where strong correlations in a many-body system play a crucial role.

The new possibilities for the single-atom-resolved probing and manipulation of ultracold quantum gases can help to achieve this goal. In particular, as shown in this thesis, the measurement of so far unattainable correlation and response functions becomes feasible. In this spirit, one of the future directions is the probing of entanglement with the goal to foster the understanding of its role in strongly interacting many-body systems $[44,45]$. 


\section{Publications}

The following articles have been published in refereed journals in the context of this thesis. The articles most relevant for this thesis are shown in bold font.

- Single-atom-resolved fluorescence imaging of an atomic Mott insulator. J. F. Sherson, C. Weitenberg, M. Endres, M. Cheneau, I. Bloch, S. Kuhr. Nature 467, 68 (2010).

- Single-spin addressing in an atomic Mott insulator.

C. Weitenberg, M. Endres, J. F. Sherson, M. Cheneau, P. Schauß, T. Fukuhara, I. Bloch, S. Kuhr.

Nature 471, 319 (2011).

- Coherent light scattering from a two-dimensional Mott insulator.

C. Weitenberg, P. Schauß, T. Fukuhara, M. Cheneau, M. Endres, I. Bloch, S. Kuhr. Phys. Rev. Lett. 106, 215301 (2011).

- Observation of Correlated Particle-Hole Pairs and String Order in LowDimensional Mott Insulators

M. Endres, M. Cheneau, T. Fukuhara, C. Weitenberg, P. Schauß, C. Gross, L. Mazza, M.C. Banuls, L. Pollet, I. Bloch, S. Kuhr

Science 334, 200 (2011)

- Light-cone-like spreading of correlations in a quantum many-body system

M. Cheneau, P. Barmettler, D. Poletti, M. Endres, P. Schauß, T. Fukuhara, C. Gross, I. Bloch, C. Kollath, S. Kuhr

Nature 481, 484 (2012)

- The 'Higgs' amplitude mode at the two-dimensional superfluid/Mott insulator transition

M. Endres, T. Fukuhara, D. Pekker, M. Cheneau, P. Schauß, C. Gross, E. Demler, S. Kuhr, I. Bloch

Nature 487, 454 (2012)

- Observation of spatially ordered structures in a two-dimensional Rydberg gas P. Schauß, M. Cheneau, M. Endres, T. Fukuhara, S. Hild, A. Omran, T. Pohl, C. Gross, S. Kuhr, I. Bloch

Nature 87, 454 (2012)

- Quantum dynamics of a mobile spin impurity.

T. Fukuhara, A. Kantian, M. Endres, M. Cheneau, P. Schauß, S. Hild, D. Bellem, U. Schollwöck, T. Giamarchi, C. Gross, I. Bloch, S. Kuhr

Nature Phys., advanced online (2013) 
The following articles have been submitted or appeared as pre-prints.

- Non-local order in Mott insulators, Duality and Wilson Loops.

S. P. Rath, W. Simeth, M. Endres, W. Zwerger arXiv:1302.0693 (2013).

- Single-site- and single-atom-resolved measurement of correlation functions. M. Endres, M. Cheneau, T. Fukuhara, C. Weitenberg, P. Schauß, C. Gross, L. Mazza, M.C. Banuls, L. Pollet, I. Bloch, S. Kuhr

Invited article for Applied Physics B, submitted (2012). 
1. Introduction 


\section{Part I.}

\section{Single-site- and single-atom-resolved detection of atomic limit Mott insulators}


The manipulation and detection of individual excitations of quantum systems forms the basis of modern quantum optics experiments [46]. However, most of these experiments have been restricted to systems composed of only a few quantum mechanically behaving objects or to weakly interacting ensembles.

Here we report on the detection of individual excitations in a strongly interacting many-body system. We demonstrate the detection of individual bosonic alkali atoms forming a Mott-insulating state in an optical lattice [3,16, 17]. The measurement is single-site and single-atom resolving and, therefore, enables detection of individual fluctuations of the many-body system.

In Ch. 2, we start out with an introduction to the Bose-Hubbard model and the quantum phase transition from a superfluid to a Mott insulator. We will explore some basic features of the phases and phase transitions in the Bose-Hubbard model and conclude with an introduction on how the model is realized using ultracold alkali atoms in optical lattices. We continue with a description of the crucial parts and steps of the experiment (Ch.3). Here, we give an introduction to the single-site-resolved detection of atoms in dilute, weakly interacting clouds and give a precise definition of the experimental observable. Next, we extend these techniques to dense, stronglyinteracting ensembles of zero-tunneling (atomic limit) Mott insulators (Ch.4). We will give a thorough introduction to this regime as it forms the starting point for all investigations in the following parts of this thesis. The main result is that reliable single-site-resolved detection of individual atoms is possible even in dense ensembles, and we are able to perform a precise, single-shot temperature measurement. 


\section{Superfluid-Mott-insulator transition}

The Hubbard model was originally developed to describe electrons in narrow conduction bands [47]. Its bosonic version, the Bose-Hubbard model [18], yields a very good description of ultracold bosonic atoms trapped in deep optical lattices, as first noted in Ref. [19]. The model shows a phase transition at zero temperature from a superfluid to a Mott-insulating phase, which forms one of the paradigm examples of a quantum phase transition [20]. The transition was first experimentally observed in 2002 using a gas of ultracold ${ }^{87} \mathrm{Rb}$ atoms in a three-dimensional optical lattice [2]. Our presentation is limited to points that are crucial for the understanding of the results presented in this thesis. The chapter also serves as a guide to the remaining thesis. For a more general introduction, see Refs. [3, 18, 20, 48].

\subsection{Bose-Hubbard model}

The Bose-Hubbard Hamiltonian is defined as [18]

$$
\hat{H}_{\mathrm{BH}}=-J \sum_{\langle i, j\rangle} \hat{b}_{j}^{+} \hat{b}_{i}+\frac{U}{2} \sum_{i} \hat{n}_{i}\left(\hat{n}_{i}-1\right)-\mu \sum_{i} \hat{n}_{i},
$$

where $\hat{b}_{i}^{\dagger}\left(\hat{b}_{i}\right)$ is the boson creation (annihilation) operator on lattice site $i, \hat{n}_{i}$ is the boson number operator, $J$ is the hopping matrix element, $U$ is the on-site interaction energy, $\mu$ is the global chemical potential, and the first sum runs over all nearest neighbors (see also Fig. 2.1). The competition between the terms gives rise to the superfluid-Mott-insulator quantum phase-transition (Fig. 2.1).

In general, quantum phase transitions describe a change in the ground state of a system as the Hamiltonian parameters are varied. Importantly, such transitions occur at zero temperature and are of a purely quantum mechanical nature (in contrast to thermal phase transitions). This can have profound consequences, such as the emergence of quantum correlations (Part II) or the existence of a Higgs amplitude mode (Part III). 


\section{Superfluid-Mott-insulator transition}

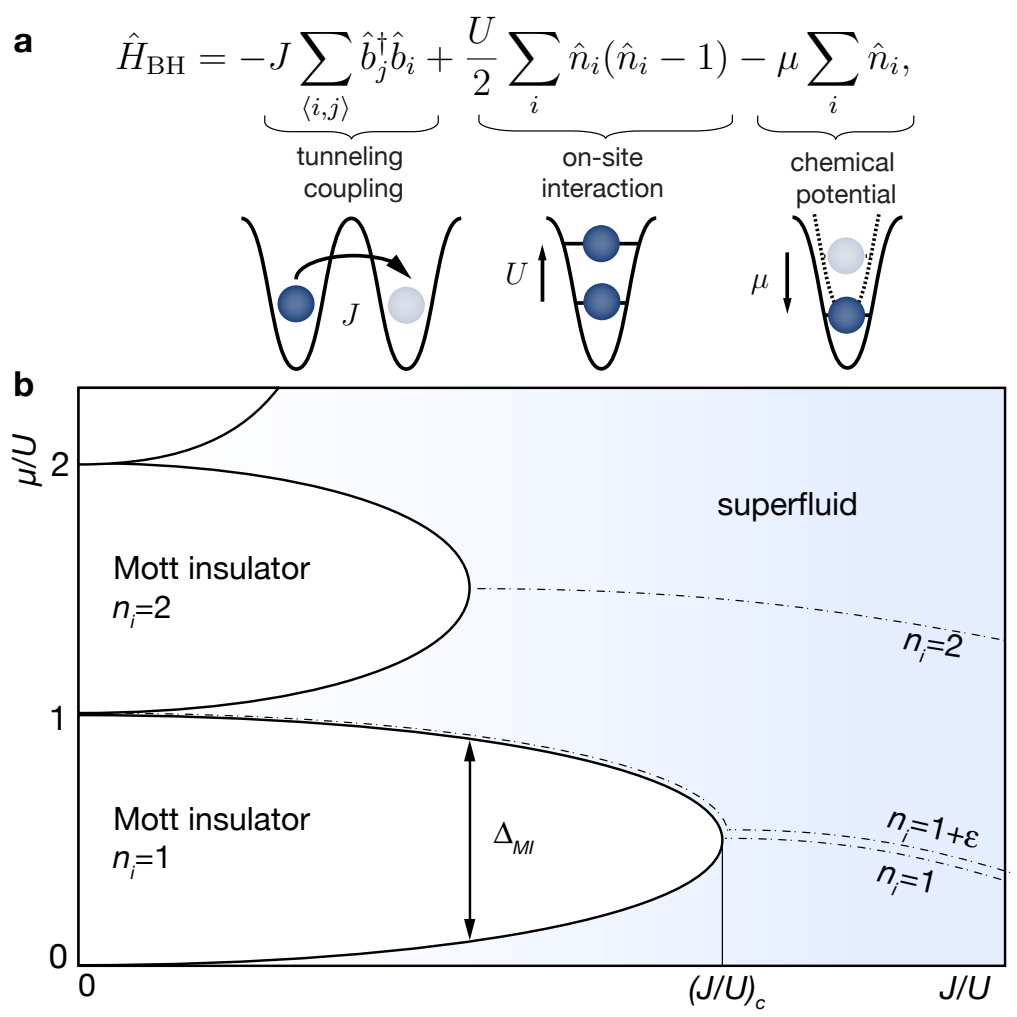

Figure 2.1.: Bose-Hubbard Hamiltonian and phase diagram. a, Illustration of the three terms in the Bose-Hubbard Hamiltonian (Eq. 2.1). The first term describes hopping between nearest neighboring sites with a hopping rate of $J / \hbar$. Due to the minus sign in front of the positive $J$, this term favors de-localization of particles. The second term describes pairwise on-site interactions and yields an energy penalty of $U$ for particles sitting on the same lattice site. The third term describes a shift of the on-site energy, which is proportional to the on-site occupation number and the chemical potential $\mu$. Due to the minus sign in front of $\mu$, this term favors higher occupation numbers. $\mathbf{b}$, Sketch of the zero temperature phase diagram of the Bose-Hubbard model [18]. For small $J / U$, the system tends to be in a Mott-insulating phase divided into lobes with constant average on-site occupation numbers $n_{i}=\left\langle\hat{n}_{i}\right\rangle$ (white areas). For $J / U$ larger than a critical value $(J / U)_{c}$, the system is always superfluid (shaded blue area) and only lines with constant $n_{i}$ exist (dashed lines). The excitation gap $\Delta_{M I}$ in the Mott-insulating phase at constant atom number is given by the width of the Mott-insulating lobes as indicated by the vertical arrow for the case $n_{i}=1$. 


\subsection{Mott-insulating and superfluid states}

\subsubsection{Mott-insulating state}

\section{Atomic limit}

The Mott-insulating state is simplest to understand in the limit of zero tunneling $J / U=0$, which we will refer to as the atomic limit. In this limit, we can neglect the tunneling term and the ground-state of the system is given by

$$
|\Psi\rangle_{J / U=0} \propto \prod_{i}\left(\hat{b}_{i}^{\dagger}\right)^{n_{i}}|0\rangle \propto \prod_{i}\left|n_{i}\right\rangle .
$$

This is a tensor product of on-site Fock states $\left|n_{i}\right\rangle$, defined as eigenstates of the on-site boson number operator $\hat{n}_{i}$, with the on-site occupation numbers $n_{i}$ as its eigenvalues (Fig.2.2). Importantly, for the state in Eq. 2.2, the variance $\sigma_{i}=\left\langle\left(\hat{n}_{i}-\left\langle\hat{n}_{i}\right\rangle\right)^{2}\right\rangle^{1 / 2}$ of the on-site occupation number vanishes.

We will discuss the physics of this limit in Ch. 4 in more detail. In particular, we will find a step-wise dependence of $n_{i}(\mu)$ on the chemical potential $\mu$, where $n_{i}(\mu)$ increases by one at integer values of $\mu / U$ and otherwise stays constant. Therefore, the state in Eq.2.2 shows incompressibility, defined as

$$
\frac{\partial\left\langle\hat{n}_{i}\right\rangle}{\partial \mu}=0,
$$

for non-integer values of $\mu / U$.

\section{Number fluctuations and incompressibility}

The ground state in the Mott-insulating regime is only given by Eq. 2.2 at $J / U=0$, and in general Mott-insulating states have a more complicated structure. Particularly, a small tunneling coupling $J$ leads to the emergence of quantum correlated particle-hole pairs, which we directly observed in the experiment (Ch.5). The effect of this is to introduce correlated number-fluctuations in the system, which lead to non-vanishing on-site fluctuations $\sigma_{i}>0$. Especially in low-dimensional systems, these number fluctuations take on significant values already in the Mott-insulating regime. Indeed, it should be stressed that the defining property of Mott insulators is incompressibility as defined in Eq. 2.3 and not $\sigma_{i}=0$ [3].

\section{Excitation structure}

In general, we find a Mott-insulating state only at integer filling, i.e., the average on-site occupation $\left\langle\hat{n}_{i}\right\rangle$ equals a non-zero integer (see dashed lines in Fig. 2.1). 


\section{Superfluid-Mott-insulator transition}

a

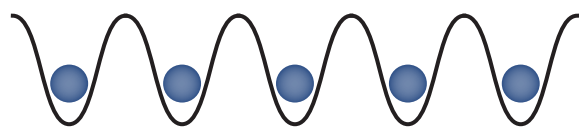

$|\Psi\rangle_{J / U=0} \propto \prod_{i}\left|n_{i}\right\rangle$

$|\Psi\rangle_{U / J=0} \propto\left(\sum_{i} \hat{b}_{i}^{\dagger}\right)^{N}|0\rangle$

b

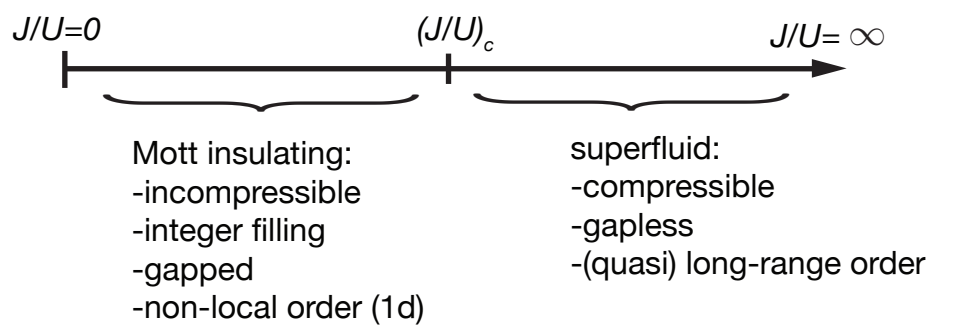

Figure 2.2.: Illustration of states in the atomic/non-interacting limit and properties of the Mott-insulating/superfluid phase. a, Sketch of the onsite-number distribution in the atomic limit $(J / U=0$, left) and the non-interacting limit $(U / J=0$, right). In the atomic limit, the atoms are strongly localized and the many-body wave function is given by a product of onsite Fock states (Eq. 2.2). In the non-interacting limit, each atom is delocalized over the entire lattice, and the many-body wave function is a product of delocalized single-particle states (Eq. 2.4). For intermediate $J / U$, the ground states have a more complicated structure (see text). $\mathbf{b}$, Summary of the main properties of Mott-insulating and superfluid states.

Additional particles that are added to the system can move (almost freely) on top of the Mott-insulating background and form a (quasi-)condensate state $[3,18]$. The same phenomenon appears when removing particles from a Mott state, leading to a condensation of holes. Thus, any deviation from integer filling immediately leads to a superfluid state.

Adding (removing) a single particle to a Mott-insulating state is associated with a finite energy cost. This energy gap is non-zero for all $J / U$ and $\mu$ values in a given Mott lobe and vanishes at the boundaries of the lobes. Therefore, the ground state within a given lobe has always the same total atom number, independent of $\mu$. Because of the translational invariance of the system, we also find that the average occupation number is independent of $\mu$, thus the state is incompressible $\frac{\partial\left\langle\hat{n}_{i}\right\rangle}{\partial \mu}=0$. We therefore find that incompressibility is a direct consequence of the finite excitation gap for adding (removing) a single particle.

The excitation gap can be directly read off from the phase diagram. At a fixed $J / U$ and $\mu / U$, the gap for adding (removing) a particle is given by the distance to the upper (lower) Mott boundary [18]. 
Additionally, we are interested in excited states at a constant total atom number. Such states are the result of a simultaneous creation of an extra particle and a hole. The corresponding excitation gap $\Delta_{M I}$ at fixed $J / U$ is given by the full width of the Mott lobe in $\mu$ direction [49] (see arrow in Fig. 2.1). The gap $\Delta_{M I}$ also sets the energy scale for thermal activation of defects in the Mott-insulating regime [18].

Increasing $J / U$, the width of the Mott lobe gradually shrinks and therefore the excitation gap $\Delta_{M I}$ is reduced until it finally closes at $J / U=(J / U)_{c}$, an effect that we could directly observe experimentally (Sec.9.2). This behavior can be intuitively understood as follows. At finite tunneling, an added particle (hole) can decrease its kinetic energy by de-localizing over the the entire system. The energy reduction becomes larger with increasing $J$. At $J / U>(J / U)_{c}$, the energy reduction of a combined particle and hole excitation, due to de-localization, overcompensates the energy cost due to the interaction term.

\section{Order parameters}

Following the previous discussion, a suitable order parameter for the Mott-insulating regime can be formulated based on the excitation gap $\Delta_{M I}$ or the compressibility $\frac{\partial\left\langle\hat{n}_{i}\right\rangle}{\partial \mu}$. With this, however, one makes use of the excited state structure or a response property. It is interesting to ask if there is a suitable order parameter which depends only on the structure of the ground state (at fixed $J / U$ and $\mu$ ). In Ch. 6, we will show that this is the case for one-dimensional systems where the order parameter can be based on non-local correlation functions that we directly observed experimentally. We will present an extension of this concept to two-dimensional systems in Ch. 7.

\subsubsection{Superfluid state}

\section{Non-interacting limit}

The superfluid phase is easiest to discuss starting from the non-interacting limit $U / J=0$. Neglecting the interaction term, the ground state is given by

$$
|\Psi\rangle_{U / J=0} \propto\left(\sum_{i} \hat{b}_{i}^{+}\right)^{N}|0\rangle,
$$

where $N$ denotes the total number of particles. The state in Eq. 2.4 shows BoseEinstein condensation in the zero-momentum Bloch state, which has a creation operator proportional to $\sum_{i} \hat{b}_{i}^{\dagger}$ (Sec.2.4). In this single particle state, an atom is de-localized over the entire lattice (Fig. 2.2). 


\section{Superfluid-Mott-insulator transition}

In the thermodynamic limit, the state in Eq. 2.4 becomes indistinguishable to a product of coherent states [3]

$$
|\Psi\rangle_{U / J=0} \propto \prod_{i}\left|\alpha_{i}\right\rangle
$$

The on-site coherent state $\left|\alpha_{i}\right\rangle$ is defined as an eigenstate of the destruction operator $\hat{b}_{i}$ with complex eigenvalue $\alpha_{i}$ [50]. The average on-site occupation number is given by $\left\langle\hat{n}_{i}\right\rangle=\left|\alpha_{i}\right|^{2}$ and we find a Poisson probability distribution to observe a certain on-site occupation number. This leads to large on-site number fluctuations $\sigma_{i}^{2}=\left\langle\hat{n}_{i}\right\rangle$. The state in Eq. 2.5 shows off-diagonal long-range order, defined as (see, e.g., [51])

$$
\lim _{|i-j| \rightarrow \infty} G^{(1)}(i, j)=\lim _{|i-j| \rightarrow \infty}\left\langle\hat{b}_{i}^{\dagger} \hat{b}_{j}\right\rangle \neq 0,
$$

where $G^{(1)}(i, j)=\left\langle\hat{b}_{i}^{\dagger} \hat{b}_{j}\right\rangle$ is the single-particle density matrix. $G^{(1)}(i, j)$ is experimentally accessible using time-of-flight imaging [3], which was used in Ref. [2] to detect the superfluid-Mott-insulator transition in a three-dimensional system.

\section{Superfluidity and long-range order}

At finite interactions $U / J>0$, the superfluid state is, in general, more complicated than the ideal Bose-Einstein condensation state in Eq. 2.4. Finite interactions lead, e.g., to a reduction of number-fluctuations, such that $\sigma_{i}^{2}<\left\langle\hat{n}_{i}\right\rangle$ already in the superfluid phase. Additionally, we would like to stress that the defining quality of the superfluid state is not long-range order (or Bose-Einstein condensation), but a nonvanishing superfluid density $n_{S F}$, when the total density of the system is written as $n=n_{S F}+n_{n}$, where $n_{n}$ is the normal component. In particular, for low-dimensional systems, long-range order in the above sense is replaced by quasi-long-range order, defined as an algebraic decay of $G^{(1)}(i, j)$. For a discussion of the relation between the behavior of $G^{(1)}(i, j)$ and superfluidity, see Refs. [3, 51].

\section{Excitation structure}

In contrast to the Mott-insulating phase, the superfluid phase, in the thermodynamic limit, does not have a finite excitation gap, i.e. it is gapless $[3,18,20,52]$. The corresponding low energy excitations are Nambu-Goldstone modes that show a linear, phonon-like dispersion relation at low momenta. In general, an excitation of these modes leads to currents of the total density $n$.

In a strongly interacting superfluid, however, additional Higgs amplitude modes, which have a non-vanishing excitation gap, can appear (Part III). In contrast to Nambu-Goldstone modes, such modes lead to variations of the superfluid density $n_{S F}$ at an almost constant overall density $n$ [49]. Similar to the excitation gap of the Mott-insulating phase, the gap to Higgs modes closes approaching the phase transition, an effect that we could directly observe experimentally (Sec. 9.2). 


\subsection{Phase transitions in the Bose-Hubbard model}

\subsection{Phase transitions in the Bose-Hubbard model}

The Bose-Hubbard model features different types of phase transitions. In particular, we find a quantum phase transition varying $J / U$ and $\mu$, but the system can also go from a superfluid to a normal phase as the temperature $T$ is increased. We will first discuss the $T=0$ transition.

At $T=0$ one can cross the superfluid-Mott-insulator boundary in different ways. First, the boundary can be crossed at constant integer density, going through the tip of the Mott lobe by varying $J / U$ (tip of lobe transition). Or, in contrast, one can vary the chemical potential at a constant $J / U$, inducing a transition that is accompanied by a change in density (generic transition). The tip of the lobe transition is special in the sense that the low-energy physics close to the transition point is described by an effectively relativistic theory [20]. The emergence of a Higgs mode in a strongly interacting superfluid close to the tip of lobe transition can be directly understood within this framework (Ch. 8). Concerning the generic transition, we already argued that an addition of particles (holes) to the Mott state with integer filling leads to a condensation of the added particles (holes). Consequently, we will see in Ch. 8 that the dynamics close to the phase boundary is essentially described by a time-dependent Gross-Pitaevskii equation.

We now turn to a description at finite $T>0$. In Fig. 2.3, we plot the phase diagram as a function of $J / U$ and $T$ at a constant integer density [20]. For low temperatures and $J / U>(J / U)_{c}$, we find a superfluid phase. Increasing $T$, the system passes through a thermal phase transition to a normal phase. The critical temperature for this transition shows a characteristic reduction close to $(J / U)_{c}$, which has been observed experimentally in Ref. [53]. Additionally, we find that the point at $T=0$ and $J / U=(J / U)_{c}$ defines a tricritical point, connected to the Mott-insulating, superfluid and normal phase.

There is no sharp phase boundary between the Mott-insulating and normal phase. Indeed, strictly speaking, the Mott-insulating state only exists at $T=0$, since for $T>0$ we always find a finite compressibility. However, for temperatures $T \ll \Delta_{M I} / k_{B}\left(k_{B}\right.$, Boltzman constant), the thermal activation of defects is strongly suppressed and characteristic properties of the Mott-insulating phase can still be observed [54]. For further details concerning the properties of the quantum phase transition, see Ref. [20].

We finish our discussion by stating the known critical values $(J / U)_{c}$ for a square lattice as a function of the dimensionality of the system in Table 2.1. In lowdimensional systems, the Mott-insulating phase reaches to higher $J / U$ values. This effect is stronger than anticipated by a mean-field approximation [18], which yields $\left(\frac{J}{U}\right)_{c, M F} \approx \frac{1}{z 5.8}$, where $z$ is the number of nearest neighbors. 


\section{Superfluid-Mott-insulator transition}

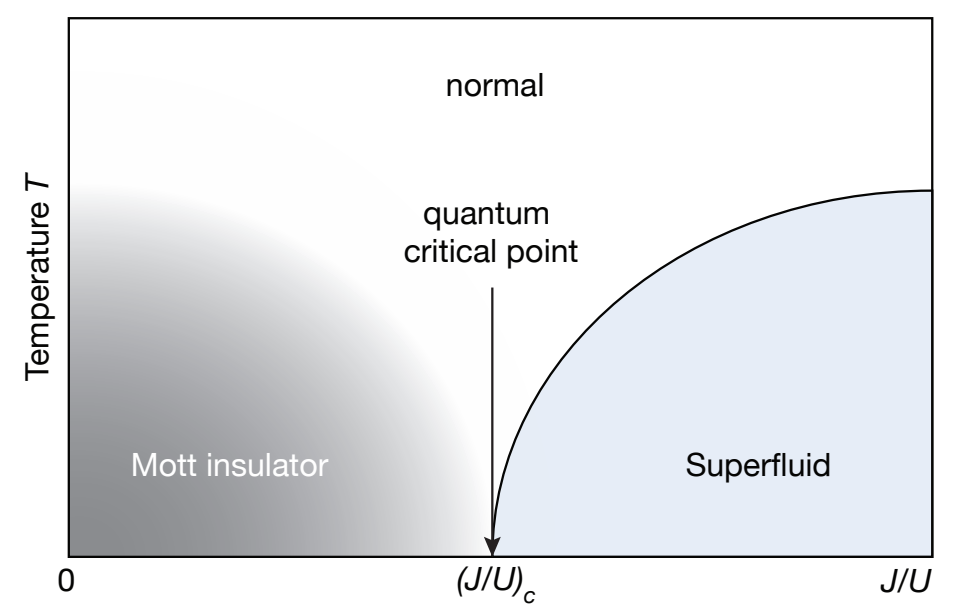

Figure 2.3.: Phase diagram at finite temperature. Figure shows a sketch of the phase diagram of the Bose-Hubbard model at constant, integer filling as a function of $J / U$ and the temperature $T$ [53]. For $J / U>(J / U)_{c}$, the system is in a superfluid phase for temperatures below a critical temperature (solid line), which shows a characteristic reduction close to $(J / U)_{c}$. For higher temperatures, we find a normal phase, which is continuously connected to the Mottinsulating phase for $J / U<(J / U)_{c}$. The point at $T=0$ and $J / U=(J / U)_{c}$ defines a tricritical point.

\begin{tabular}{|c||c|c|c|}
\hline & $d=3$ & $d=2$ & $d=1$ \\
\hline \hline$(J / U)_{c} \approx$ & $0.034[55]$ & $0.059[56]$ & $0.3[57,58]$ \\
\hline$(J / U)_{c, M F} \approx \frac{1}{z 5.8} \approx$ & 0.029 & 0.043 & 0.086 \\
\hline
\end{tabular}

Table 2.1.: Critical value $(J / U)_{c}$ for the superfluid-Mott-insulator transition. Top row: critical values $(J / U)_{c}$, based on numerical calculations for the transition in dimensions $d=$ $3,2,1$. Bottom row: Same as top row, but based on a mean-field calculation. The mean-field values underestimate $(J / U)_{c}$, particularly for $d=2,1$. 


\subsection{Realization of the Bose-Hubbard model in optical lattices}

We give a short introduction on how the Bose-Hubbard model can be realized with ultracold alkali atoms in an optical lattice. Very detailed descriptions can be found in Refs. [3, 59-62]. As the three-dimensional lattice potential, in our case, is separable, we first deal with a single lattice axis. The full solution is a simple extension of these results (Sec. 2.4.3).

The starting point of our discussion is the general many-body Hamiltonian

$$
\begin{aligned}
\hat{H} & =\int d x \hat{\Psi}^{\dagger}(x)\left(-\frac{\hbar^{2}}{2 m} \frac{\partial^{2}}{\partial x^{2}}+V_{l}(x)-\mu\right) \hat{\Psi}(x) \\
& +\frac{1}{2} \int d x d x^{\prime} \hat{\Psi}^{\dagger}(x) \hat{\Psi}^{\dagger}\left(x^{\prime}\right) V\left(x-x^{\prime}\right) \hat{\Psi}\left(x^{\prime}\right) \hat{\Psi}(x)
\end{aligned}
$$

for spinless bosons interacting via $V\left(x-x^{\prime}\right)$ in the presence of an external potential $V_{l}(x)$ (see, e.g., Ref. [63]). $\hat{\Psi}^{\dagger}(x)(\hat{\Psi}(x))$ are creation (annihilation) operators at position $x$, with commutation relations $\left[\hat{\Psi}(x), \hat{\Psi}^{\dagger}\left(x^{\prime}\right)\right]=\delta\left(x-x^{\prime}\right)$.

\subsubsection{Optical lattice potential}

An optical lattice, in its simplest form, is created by a pair of counter-propagating laser beams with the same laser wavelength $\lambda_{L}$. This leads to a standing wave along the propagation direction, which has a periodically varying intensity profile $I(x)$ proportional to $\cos \left(k_{\text {lat }} x\right)$, where $k_{\text {lat }}=\frac{2 \pi}{a_{\text {lat }}}$ and $a_{\text {lat }}=\lambda_{L} / 2$ (Fig. 2.4).

The lattice light creates a conservative potential $V_{l}(x)$ for the atoms via the dynamical Stark effect (or a.c. Stark effect). A comparison of different treatments for this effect can be found in Ref. [64]. For alkali atoms, the potential $V_{l}(x)$ resulting from linearly polarized light and detunings that do not resolve the hyperfine structure is [65]

$$
V_{\mathrm{L}}(x)=-\frac{\pi c^{2}}{2 \omega_{\mathrm{D} 2}^{3}} \frac{1}{\tau_{\mathrm{D} 2}}\left(\frac{2}{\Delta_{\mathrm{D} 2}}+\frac{1}{\Delta_{\mathrm{D} 1}}\right) I(x),
$$

where $c$ is the speed of light and $\tau_{\mathrm{D} 2}$ is the lifetime of the excited state of the D2 transition. The effective detunings $\Delta_{D 1 / D 2}$ are given by

$$
\frac{1}{\Delta_{\mathrm{D} 1 / \mathrm{D} 2}}=\frac{1}{\left(\omega_{\mathrm{D} 1 / \mathrm{D} 2}-\omega_{L}\right)}+\frac{1}{\left(\omega_{\mathrm{D} 1 / \mathrm{D} 2}+\omega_{L}\right)},
$$

with $\omega_{L}=2 \pi \frac{c}{\lambda_{L}}$ and $\omega_{\mathrm{D} 1 / \mathrm{D} 2}=2 \pi \frac{c}{\lambda_{\mathrm{D} 1 / \mathrm{D} 2}}$, where $\lambda_{\mathrm{D} 1 / \mathrm{D} 2}$ is the transition wavelength of the D1 (D2) line respectively.

The resulting potential $V_{L}(x)$ is proportional to $\pm I(x)$, for effective blue (red) detuning, i.e. the term $\frac{2}{\Delta_{\mathrm{D} 2}}+\frac{1}{\Delta_{\mathrm{D} 1}}$ is negative (positive). We thus find a periodic lattice 


\section{Superfluid-Mott-insulator transition}

potential

$$
V_{l}(x)= \pm \frac{V_{0}}{2} \cos \left(k_{\text {lat }} x\right)
$$

where we have appropriately chosen an absolute energy offset and the overall phase of the periodic potential. For blue (red) detuning, the minima of $V_{l}(x)$ coincide with the minima (maxima) of the intensity distribution $I(x) . V_{0}$ is the full lattice depth, which is typically stated in units of the lattice recoil energy $E_{r}=h^{2} /\left(8 m a_{\text {lat }}^{2}\right)$, where $m$ is the atomic mass. In the case of ${ }^{87} \mathrm{Rb}$ and $\lambda_{L}=1064 \mathrm{~nm}$, we find $E_{r} / h \approx 2 \mathrm{kHz}$.

\subsubsection{Derivation of the tunneling and interaction term}

\section{Tunneling term}

The energy eigenstates of the single-particle Hamiltonian $\hat{H}_{s}=-\frac{\hbar^{2}}{2 m} \frac{\partial^{2}}{\partial x^{2}}+V_{l}(x)$, with $V_{l}(x)$ defined in Eq. 2.10, are Bloch functions $\Phi_{q}(x)$ with eigenenergies $\epsilon(q)$, where $q$ is a quasi-momentum $|q|<\frac{\pi}{a_{\text {lat }}}$ restricted to the first Brillouin zone (Fig. 2.4). For an introduction to periodic potentials, we refer to Ref. [66]. We will restrict ourself to the lowest Bloch band and suppress a band index.

The Bloch functions $\Phi_{q}(x)$ and the eigenenergies $\epsilon(q)$ can, in general, be calculated with a numerical band-structure calculation [59]. Alternatively, one can show that the eigenvalue equation $\hat{H}_{S} \Psi(x)=E \Psi(x)$ is equivalent to the Mathieu equation, which has Mathieu cosine and Mathieu sine functions as its unique even and odd solutions [67]. Following Ref. [68], one can write the Bloch functions $\Phi_{q}(x)$ as a linear combination of the latter functions.

The key concept for the derivation of the Bose-Hubbard model is the Wannier function $w_{i}(x)$, defined by a Fourier transform of the Bloch functions [69]

$$
w_{i}(x)=\frac{1}{\sqrt{N_{l}}} \sum_{q} \Phi_{q}(x) e^{-i q x_{i}}
$$

where $x_{i}$ refers to a lattice site $i$, defined by the minima of the lattice potential $V_{l}(x)$. The sum runs over the first Brillouin zone and $N_{l}$ is the number of lattice sites.

The Wannier functions $w_{i}(x)$ of the lowest band have the following important properties. First, they are only functions of the distance $x-x_{i}$. Additionally, they can be chosen to be symmetric around $x_{i}$ and to be real [68]. Furthermore, they form a complete, orthogonal set for the lowest band. For sufficiently deep lattices, they are strongly localized around $x_{i}$ (Fig. 2.4).

The crucial step in the derivation of the Bose-Hubbard model is the expansion of the 


\subsection{Realization of the Bose-Hubbard model in optical lattices}

a

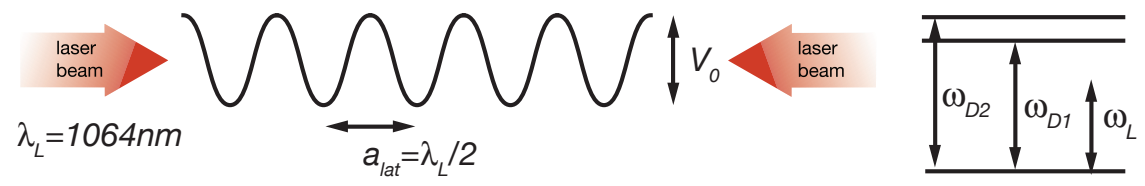

b

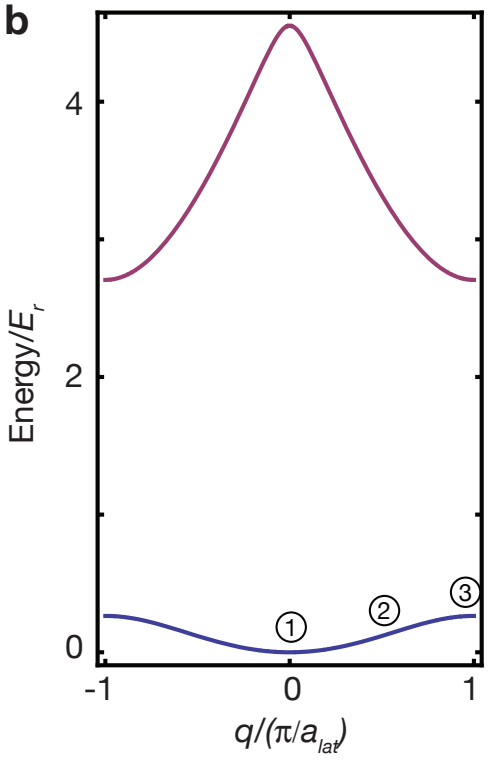

C

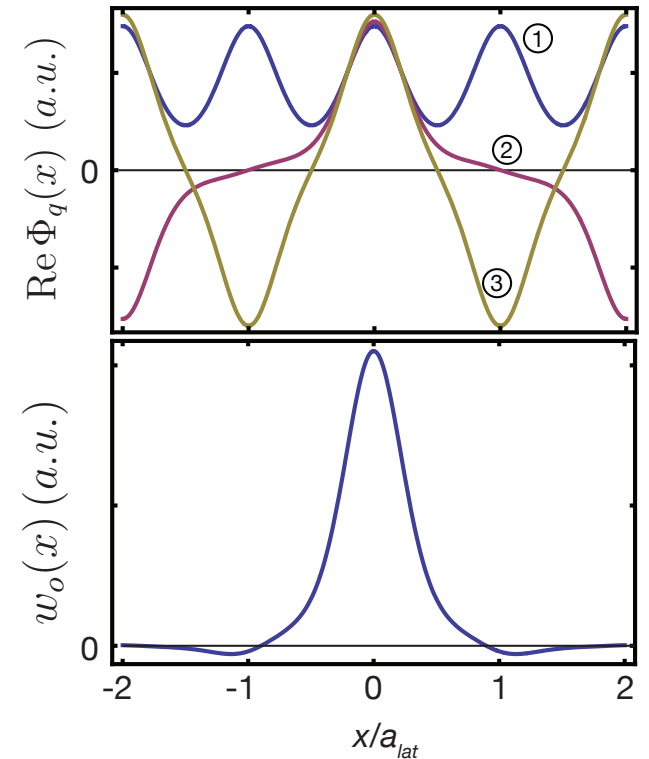

Figure 2.4.: Realizing the Bose-Hubbard model using optical lattices. a, Illustration of an optical lattice potential. Two counter-propagating laser beams with wavelength $\lambda_{L}$ interfere, and form a standing wave with period $a_{\text {lat }}=\lambda_{L} / 2$. The laser frequency $\omega_{L}=2 \pi \frac{c}{\lambda_{\mathrm{L}}}$ is chosen to be far off-resonant to the D1 and D2 lines, resulting in an conservative trapping potential due to the dynamical Stark effect. $\mathbf{b}$, Band structure for a lattice depth of $V_{0}=5 E_{r}$ including the lowest (blue) and the first excited (red) band. c, Real part of the Bloch wave functions $\Phi_{q}(x)$ for the lowest band for quasi-momenta $q=0, \pi /\left(2 a_{\text {lat }}\right), \pi / a_{\text {lat }}$ (blue, red, yellow; see corresponding numbers in $\mathbf{b})$. $\mathbf{d}$, Wannier function of the lowest band $w_{0}(x) \propto \sum_{q} \Phi_{q}(x)$ for the central lattice site at $x_{i}=0$. The summation of Bloch wave functions with different momenta leads to a constructive interference around $x=0$ and to a destructive interference at neighboring lattice sites. The Wannier function is completely real, because the imaginary parts of $\Phi_{q}(x)$ destructively interfere at all positions (not shown).

boson creation (annihilation) operators in terms of Wannier functions given by

$$
\begin{aligned}
\hat{\Psi}^{\dagger}(x) & =\sum_{i} w_{i}^{*}(x) \hat{b}_{i}^{\dagger} \\
\hat{\Psi}(x) & =\sum_{i} w_{i}(x) \hat{b}_{i}
\end{aligned}
$$




\section{Superfluid-Mott-insulator transition}

where $\hat{b}_{i}^{\dagger}\left(\hat{b}_{i}\right)$ are the creation (annihilation) operators for a state with Wannier wave function $w_{i}(x)$.

Due to the completeness of $w_{i}(x), \hat{b}_{j}$ and $\hat{b}_{i}^{\dagger}$ fulfill the commutation relations

$$
\left[\hat{b}_{i}, \hat{b}_{j}^{\dagger}\right]=\delta_{i, j} .
$$

With Eq. 2.13, we can write the non-interacting part $\hat{H}_{0}$ of $\hat{H}$ as

$$
\begin{aligned}
\hat{H}_{0} & =\int d x \hat{\Psi}^{\dagger}(x)\left(-\frac{\hbar^{2}}{2 m} \frac{\partial^{2}}{\partial x^{2}}+V_{l}(x)-\mu\right) \hat{\Psi}(x) \\
& =-\sum_{i \neq j} J_{i, j} \hat{b}_{i}^{+} \hat{b}_{j}-\sum_{i}\left(\mu+J_{i, i}\right) \hat{b}_{i}^{\dagger} \hat{b}_{i} \\
& =-\sum_{i \neq j} J_{i, j} \hat{b}_{i}^{+} \hat{b}_{j}-\mu \sum_{i} \hat{b}_{i}^{+} \hat{b}_{i} .
\end{aligned}
$$

In the last line, we absorbed $J_{i, i}$ in the chemical potential $\mu$, by replacing $\mu \rightarrow \mu-J_{i, i}$. The tunneling matrix element $J_{i, j}$ is given by

$$
J_{i, j}=-\int d x w_{i}^{*}(x)\left(-\frac{\hbar^{2}}{2 m} \frac{\partial^{2}}{\partial x^{2}}+V_{l}(x)\right) w_{j}(x) .
$$

The first term in Eq. 2.15 describes hopping between arbitrary lattice sites with hopping matrix element $J_{i, j}$. In deep lattices, $J_{i, j}$ is negligible for all, but nearest neighbors. Defining $J=J_{i, i+1}$ and neglecting all other $J_{i, j}$, we arrive at

$$
\hat{H}_{0} \approx-J \sum_{\langle i, j\rangle} \hat{b}_{i}^{\dagger} \hat{b}_{j}-\mu \sum_{i} \hat{b}_{i}^{\dagger} \hat{b}_{i}
$$

resembling the non-interacting terms in the Bose-Hubbard model.

\section{Interaction term}

At sufficiently low temperatures, interactions between bosonic alkali atoms are restricted to s-wave collisions. The scattering wavefunction, resulting from the true interaction potential, is at low collisional momenta correctly reproduced by a delta function contact potential $[3,70,71]$

$$
V\left(\vec{r}-\vec{r}^{\prime}\right)=\frac{4 \pi \hbar^{2} a_{s}}{m} \delta\left(\vec{r}-\vec{r}^{\prime}\right)=g \delta\left(\vec{r}-\vec{r}^{\prime}\right) .
$$

The s-wave scattering length $a_{S}$ takes on a value $a_{s}=100.4(1) a_{B}\left(a_{B} \approx 5.29 \cdot 10^{-11} \mathrm{~m}\right.$, the Bohr radius) for collisions between ${ }^{87} \mathrm{Rb}$ atoms in the hyperfine state 
$5 S_{1 / 2}, F=1, m_{F}=-1$ used in our experiments [72]. The interaction strength is defined as $g=\frac{4 \pi \hbar^{2} a_{s}}{m}$. We do not discuss additional regularization terms [70].

We would like to deal with a single lattice axis first, but the previous formula defines the interaction potential in three dimensions. To be formally correct and to yield the right units, we define a one-dimensional interaction potential $V_{1 \mathrm{~d}}\left(x-x^{\prime}\right)=g_{1 \mathrm{~d}} \delta\left(x-x^{\prime}\right)_{1 \mathrm{~d}}$, with a one-dimensional interaction strength $g_{1 \mathrm{~d}}=g \cdot\left(\int d x\left|w_{i}(x)\right|^{4}\right)^{2}$ and a one-dimensional delta function $\delta\left(x-x^{\prime}\right)_{1 \mathrm{~d}}$. We will deal with the full three-dimensional problem in the next subsection and the factor $\left(\int d x\left|w_{i}(x)\right|^{4}\right)^{2}$ will be motivated there.

With this at hand, we can write the interaction part $\hat{H}_{I}$ of $\hat{H}$ as

$$
\begin{aligned}
\hat{H}_{I} & =\frac{1}{2} \int d x d x^{\prime} \hat{\Psi}^{\dagger}(x) \hat{\Psi}^{\dagger}\left(x^{\prime}\right) V\left(x-x^{\prime}\right)_{1 \mathrm{~d}} \hat{\Psi}\left(x^{\prime}\right) \hat{\Psi}(x) \\
& =\frac{g 1 \mathrm{~d}}{2} \int d x \hat{\Psi}^{\dagger}(x) \hat{\Psi}^{\dagger}(x) \hat{\Psi}(x) \hat{\Psi}(x)
\end{aligned}
$$

Inserting the expansion in terms of Wannier functions (Eq. 2.13) yields

$$
\begin{aligned}
\hat{H}_{I} & \approx \frac{U}{2} \hat{b}_{i}^{+} \hat{b}_{i}^{+} \hat{b}_{i} \hat{b}_{i} \\
& =\frac{U}{2} \hat{b}_{i}^{\dagger} \hat{b}_{i}\left(\hat{b}_{i}^{+} \hat{b}_{i}-1\right),
\end{aligned}
$$

with the on-site interaction $U$ given by

$$
U=g_{1 \mathrm{~d}} \int d x\left|w_{i}(x)\right|^{4} .
$$

In the first line of Eq.2.20, we assumed that $\left.\left|\int d x\right| w_{i}(x)\right|^{4} \mid \gg$ $\left|\int d x w_{k}(x) w_{l}(x) w_{m}(x) w_{n}(x)\right|$, if at least one of the indices $k, l, m, n$ differs from the others. This is justified, when the Wannier functions are strongly localized and the overlap between neighboring sites is therefore small.

\subsubsection{Three-dimensional optical lattice and harmonic confinement}

A three-dimensional optical lattice, in our case, is created by three pairs of counterpropagating laser beams intersecting at right angles (Ch.3). All beams are chosen to have the same laser wavelength and the overall potential thus resembles a simple cubic lattice.

Additionally, all beams have an intrinsic Gaussian beam shape, which can be approximated by a harmonic potential in the beam's center. The overall lattice potential can 


\section{Superfluid-Mott-insulator transition}

be written as [59]

$$
\begin{aligned}
& V_{l}(\vec{r}) \approx V_{p}(\vec{r})+V_{h}(\vec{r}) \\
& V_{p}(\vec{r})= \pm\left(\frac{V_{x}}{2} \cos \left(k_{\text {lat }} r_{x}\right)+\frac{V_{y}}{2} \cos \left(k_{\text {lat }} r_{y}\right)+\frac{V_{z}}{2} \cos \left(k_{\text {lat }} r_{z}\right)\right) \\
& V_{h}(\vec{r})= \pm\left(\frac{1}{2} m \omega_{x}^{2} r_{x}^{2}+\frac{1}{2} m \omega_{y}^{2} r_{y}^{2}+\frac{1}{2} m \omega_{z}^{2} r_{z}^{2}\right),
\end{aligned}
$$

where $\vec{r}=\left(r_{x}, r_{y}, r_{z}\right)$ is the position, $V_{x}, V_{y}, V_{z}$ are the full lattice depths in $x, y, z-$ directions and $\omega_{x}, \omega_{y}, \omega_{z}$ are the trapping frequencies in $x, y, z$-directions. We therefore find a separable periodic potential $V_{p}(\vec{r})$ and a harmonic potential $V_{h}(\vec{r})$.

The previous one-dimensional calculation is easily extended to the three-dimensional case. The band structure calculation for each axis yields Wannier functions $w_{i}^{x}\left(r_{x}\right), w_{i}^{y}\left(r_{y}\right), w_{i}^{z}\left(r_{z}\right)$ for the $x, y, z$ axis respectively. Since the periodic potential is separable, the Wannier function $w_{i}(\vec{r})$ for the three-dimensional case is given by

$$
w_{i}(\vec{r})=w_{i}^{x}\left(r_{x}\right) w_{i}^{y}\left(r_{y}\right) w_{i}^{z}\left(r_{z}\right),
$$

which replaces $w_{i}(x)$ in the above calculation.

In total, we find

$$
\hat{H} \approx-\sum_{\langle i, j\rangle} J_{\alpha} \hat{b}_{i}^{\dagger} \hat{b}_{j}+\frac{U}{2} \sum_{i} \hat{n}_{i}\left(\hat{n}_{i}-1\right)-\sum_{i} \mu_{i} \hat{b}_{i}^{\dagger} \hat{b}_{i},
$$

where we absorbed the terms resulting from the harmonic potential $V_{h}(\vec{r})$ in a local chemical potential $\mu_{i}$ given by

$$
\mu_{i}=\mu-V_{h}\left(\vec{r}_{i}\right) .
$$

The resulting model in Eq. 2.26 is usually treated in a local density approximation, which assumes that the solution in the homogeneous case at a given constant $\tilde{\mu}$ reproduces the local physics in the spatially varying problem at $\mu_{i}=\tilde{\mu}$ (see, e.g., Ref. [73] for a discussion). This treatment becomes exact in the atomic limit (Ch. 4).

Additionally, we introduced a hopping matrix element $J_{\alpha}$ along the different axes, where $\alpha=x, y$ or $z$ depending on whether the nearest neighbors $i, j$ are aligned in $x, y$ or $z$ direction, respectively. The hopping matrix element $J_{\alpha}$ is given by

$$
\begin{aligned}
J_{\alpha} & =-\int d r_{\alpha} w_{i}^{\alpha}\left(r_{\alpha}\right)^{*}\left(-\frac{\hbar^{2}}{2 m} \frac{\partial^{2}}{\partial r_{\alpha}^{2}}+\frac{V_{\alpha}}{2} \cos \left(k_{\mathrm{lat}} r_{\alpha}\right)\right) w_{i+1}^{\alpha}\left(r_{\alpha}\right) \\
& =\frac{1}{N_{l}} \sum_{|q|<\frac{\pi}{a_{\mathrm{lat}}}} e^{i q a_{\mathrm{lat}}} \epsilon^{\alpha}(q)
\end{aligned}
$$



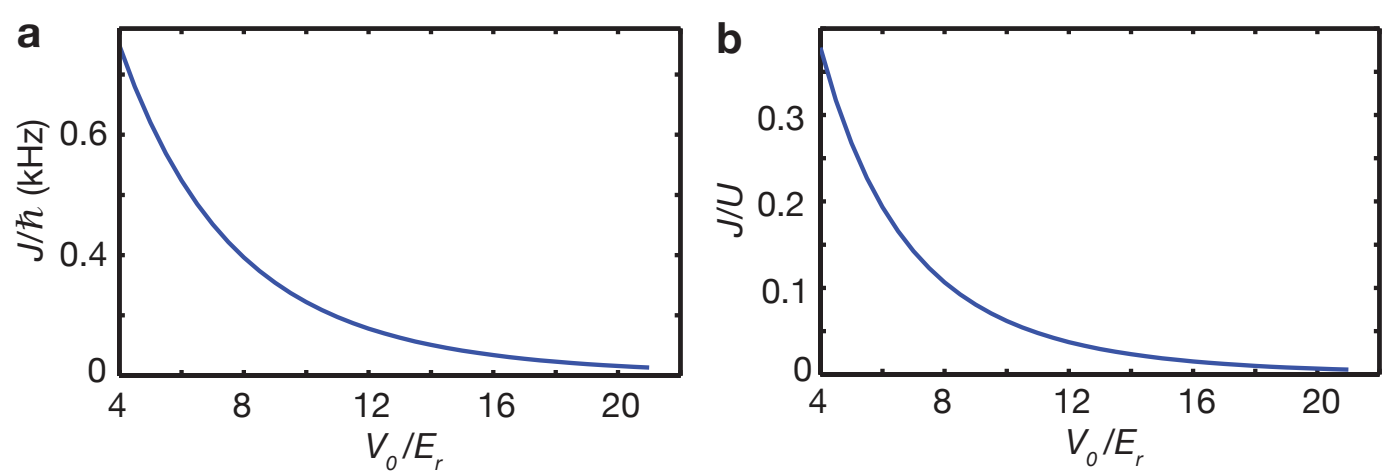

Figure 2.5.: Bose-Hubbard parameters as a function of the lattice depth. Bose-Hubbard parameters as a function of the lattice depth $V_{0}$ in units of the lattice recoil energy $E_{r}$. a, The plot shows that $J$ falls off approximately exponentially with the lattice depth $V_{0}$. $\mathbf{b}$, The behavior of $J / U$ is dominated by the strong dependence of $J$ on $V_{0}$. For the calculation of $J / U$ shown in this plot, we kept the depths of the two orthogonal axes constant at $17 E_{r}$ and $20 E_{r}$ resembling the situation described in Sec.5.2. For the calculation, we used $a_{\text {lat }}=532 \mathrm{~nm}$

where in the last line, we inserted the definition of the Wannier functions (Eq. 2.13) and used the fact that the Bloch functions are eigenstates of $-\frac{\hbar^{2}}{2 m} \frac{\partial^{2}}{\partial r_{\alpha}^{2}}+\frac{V_{\alpha}}{2} \cos \left(k_{\text {lat }} r_{\alpha}\right)$ with eigenvalues $\epsilon^{\alpha}(q)$.

The on-site interaction $U$ is given by

$$
U=g \int d r_{x}\left|w_{i}^{x}\left(r_{x}\right)\right|^{4} \int d r_{y}\left|w_{i}^{y}\left(r_{y}\right)\right|^{4} \int d r_{z}\left|w_{i}^{z}\left(r_{z}\right)\right|^{4} .
$$

In Fig. 2.5, we show $J$ and $U$, determined from the previous formulas using a bandstructure calculation for typical experimental parameters.

Finally, we discuss that the definition of the Wannier functions is to some degree ambiguous. The problem lies in the fact that the Bloch wavefunctions can be multiplied by a $q$ dependent phase factor, such that $\Phi_{q}(x) \rightarrow e^{i \phi(q)} \Phi_{q}(x)$, which does not change their properties. However, the phase factor enters the Fourier transform used for the definition of the Wannier function in Eq. 2.11 and, in general, changes the resulting Wannier function.

In practice, one is interested in Wannier functions, which are maximally localized at a single lattice site. The conditions for maximally localized Wannier functions for general one-dimensional periodic potentials have been investigated in Ref. [68]. In our case, to yield maximally localized Wannier functions for the lowest band, the phases for the Bloch functions have to be fixed such that

$$
\operatorname{Im}\left(e^{i \phi(q)} \Phi_{q}(0)\right)=0,
$$




\section{Superfluid-Mott-insulator transition}

where Im denotes the imaginary part.

From Eq. 2.29, we see that this does not affect the value of the tunneling matrix element as only the band-structure enters the formula. In contrast, the on-site interaction does depend on the choice of the phase factor (Eq. 2.30).

\subsubsection{Discussion}

Here we summarize the assumptions that led to the derivation of Eq. 2.26 and verify that they are justified for the experimental values in this thesis.

First, we restricted ourself to the lowest Bloch band, which is justified if the thermal and interaction energies are smaller than the band gap (Fig. 2.4). Typical interaction energies, in our case, are about $U / h \approx 500 \mathrm{~Hz}$ and we find temperatures $T \ll U / k_{b}$ (Ch. 4). Both energies are therefore significantly smaller than the band gap of about $4-16 \mathrm{kHz}$ for our typical lattice depths of about $4-21 E_{r}$.

Additionally, we only accounted for nearest neighbor tunneling, i.e., we made a tight-binding approximation. For our typical lattice depths, the tunneling matrix element to the next-nearest neighbor is about a factor of $10-1000$ smaller than the one for nearest neighbor tunneling and the assumption is therefore legitimate.

Furthermore, we assumed pure on-site interactions, which is justified in our case because nearest-neighbor interactions are suppressed by at least a factor of 200.

Finally, the on-site interaction $U$ might show a small dependence on the on-site occupation number that we neglected (see, e.g., Ref. [62] for a discussion).

The main control knobs in our experiment are the lattice depths $V_{x}, V_{y}, V_{z}$, which can be tuned to reach different Bose-Hubbard parameters $J_{x}, J_{y}, J_{z}$ and $U$. In this section, we showed that these parameters can be determined from knowledge of the band structure including the Bloch waves $\Phi_{q}(x)$ with eigenenergies $\epsilon(q)$. The band structure can be easily numerically calculated after the lattice depths $V_{x}, V_{y}, V_{z}$ have been determined experimentally (App. A).

In conclusion, we find an exceptional situation where the many-body Hamiltonian is known, and the Hamiltonian parameters can be controlled and determined with a high degree of accuracy. 


\section{Overview of the experimental procedure}

This chapter gives an overview of the experimental setup and sequence. The aim is to give a general introduction to the core parts of the sequence and the high-resolution imaging of single atoms. At the end of the chapter, we additionally discuss the most general observable detectable with the latter technique. The description is limited to information that is essential to understanding and interpreting the experimental results described in the following chapters. A more detailed account can be found in Ref. [74] and App. A.

\subsection{Two-dimensional degenerate gas and vertical lattice}

The starting point of the relevant part of the experimental sequence was a twodimensional degenerate quantum gas consisting of several hundred ${ }^{87} \mathrm{Rb}$ atoms in the hyperfine state $5 S_{1 / 2}, F=1, m_{F}=-1$ [75]. It was prepared in a single anti-node of an optical lattice in the vertical direction (Fig.3.1a). Details of the preparation procedure are described in App. A.1.

The vertical lattice was generated by interference between an incoming laser beam and its reflection from a vacuum window, which has a high-reflectivity coating for the laser wavelength $\lambda_{\mathrm{L}}=1064 \mathrm{~nm}$. The 90-degree incident angle on the window resulted in a lattice spacing of $a_{\text {lat }}=\lambda_{\mathrm{L}} / 2=532 \mathrm{~nm}$. The laser frequency was reddetuned to the D2 and D1 line, yielding an attractive ac Stark potential (Sec. 2.4).

The lattice depth $V_{z}$ was kept constant at approximately $21 E_{r}$, where $E_{r}$ denotes the lattice recoil energy $E_{r}=h^{2} /\left(8 m a_{\text {lat }}^{2}\right)$ with $m$ the atomic mass of ${ }^{87} \mathrm{Rb}$. Due to this tight confinement and an additional energy offset from gravity, tunnelling of the atoms in the vertical direction was negligible for the duration of the experiment. Additionally, the vertical confinement was much stronger than the harmonic confinement in horizontal direction (Sec. 2.4). In this pancake geometry, the atom cloud formed a two-dimensional degenerate Bose gas [76-82]. 


\section{Overview of the experimental procedure}
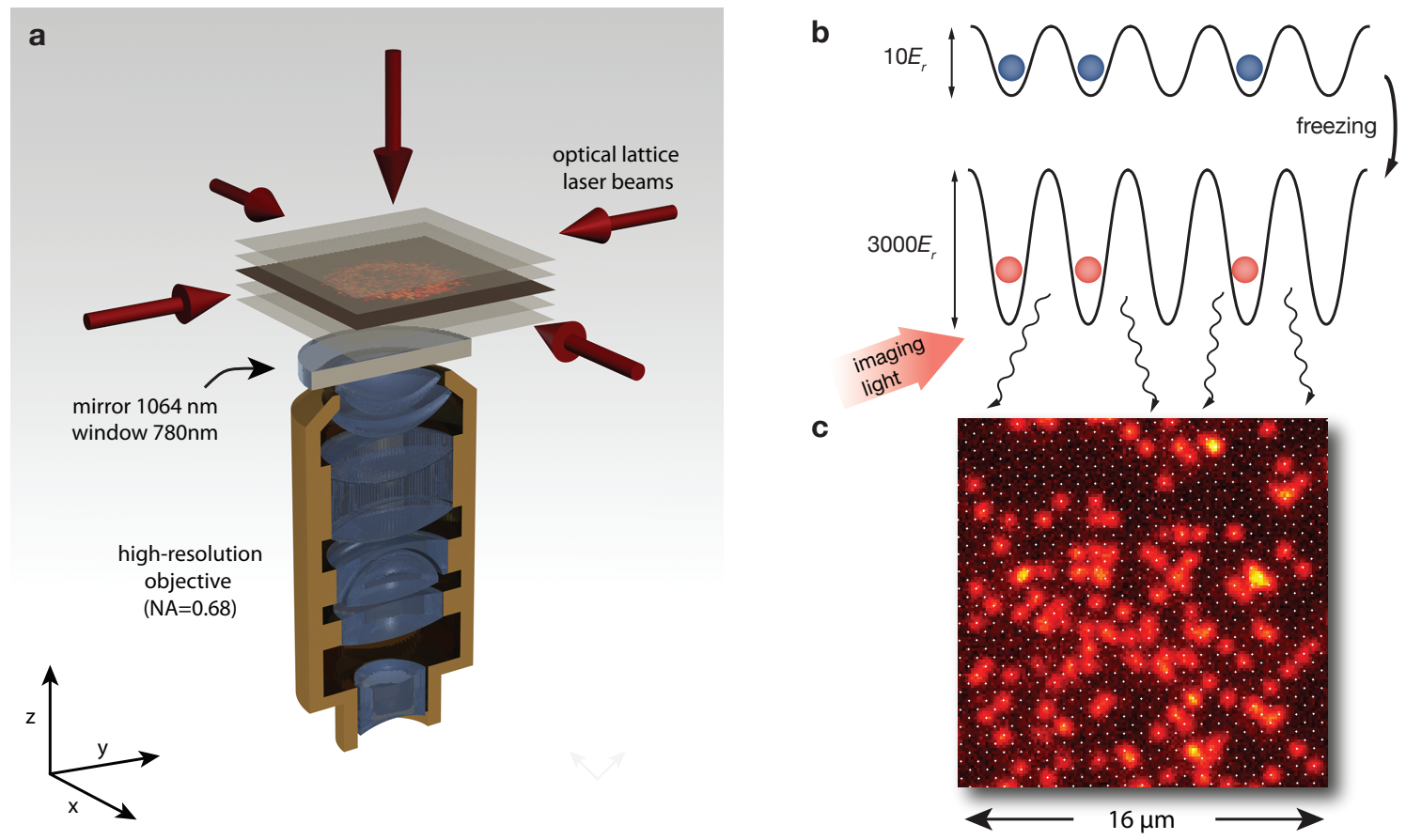

Figure 3.1.: Experimental setup and fluorescence imaging. a, Schematic image showing the optical lattice geometry, the two-dimensional quantum gas, and the high-resolution objective [17]. The optical lattice setup consisted of two horizontal lattice axes (in the $x$ and $y$-directions) and a vertical lattice axis (in the $z$-direction). The latter was created by reflection from a vacuum window with a high-reflectivity coating for the lattice wavelength $(1064 \mathrm{~nm})$ and a transmission coating for the imaging wavelength $(780 \mathrm{~nm})$. The two-dimensional quantum gas was prepared in a single anti-node of the vertical lattice and is imaged with the highresolution objective using fluorescence detection. $\mathbf{b}$, The gas is typically kept in a moderately deep horizontal optical lattice of about $10 E_{r}$ (Sec.2.4). For the imaging, we froze the density distribution by rapidly increasing all lattice depths to about $3000 E_{r}$. The near-resonant imaging light is scattered by the atoms and collected with the high-resolution objective on an emccd (electron-multiplying charge-coupled device) camera. c, Typical fluorescence image of a dilute thermal cloud as seen on the emccd camera. Single atoms (red dots) are visible with a high signal-to-noise ratio. White dots mark the sites of the two-dimensional lattice created by the horizontal lattice axes.

\subsection{Horizontal square lattice and adiabatic preparation}

Following the previously described preparation, the gas was loaded into a twodimensional optical lattice formed by two lattice axes in the horizontal direction (Fig.3.1), which were created by reflections from mirrors outside of the vacuum chamber. The lattice spacing in both directions was $a_{\text {lat }}=\lambda_{\mathrm{L}} / 2=532 \mathrm{~nm}$ and the 


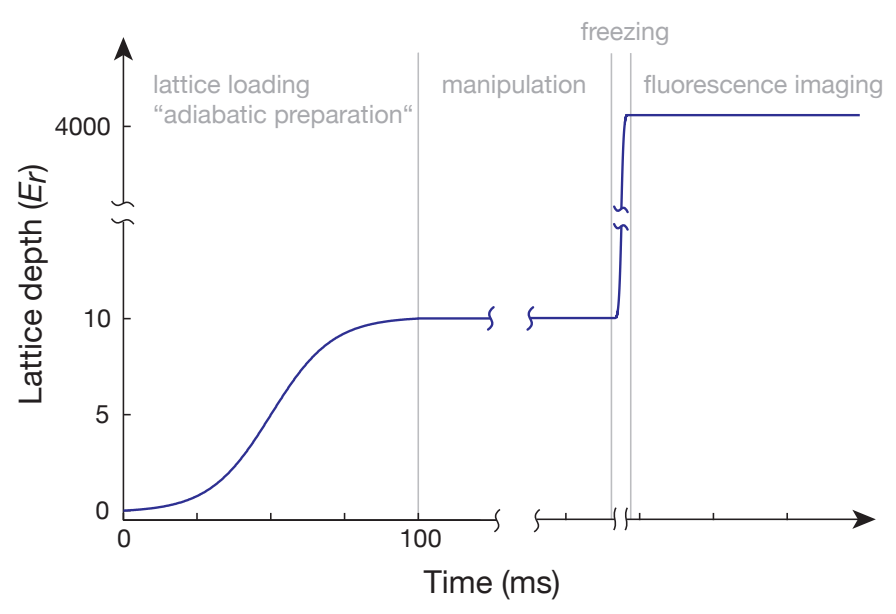

Figure 3.2.: Typical sequence. Horizontal lattice depth during a typical experimental sequence. After preparation in a single anti-node of the vertical lattice, we superimposed a horizontal square-lattice. The loading into the square-lattice consisted of a quasi-adiabatic s-shaped ramp with a duration of $75-120 \mathrm{~ms}$. This was followed by a possible manipulation phase (e.g., lattice modulation). For the detection, the lattice gas is frozen by a fast ramp of the lattice depth to approximately $3000 E_{r}$.

axes intersected at a right angle resulting in a simple two-dimensional square lattice. For the loading, both lattice depths were increased to 7-23 $E_{r}$ following s-shaped functions with durations of 75-120 ms (Fig. 3.2).

These lattice ramps were quasi-adiabatic, i.e. slow enough to keep the system close to its many-body ground state, as we will see later by the low defect density in the system (Ch.4). The exact shape of these ramps and the final lattice depths were the key experimental parameters used to change the equilibrium state of the lattice gas before the actual measurement was performed. In the following, we will use the term adiabatic preparation for the aforementioned loading process. The combined harmonic potential of all three lattices leads to trapping frequencies of typically $\omega /(2 \pi) \approx 60 \mathrm{~Hz}$.

\subsection{Fluorescence imaging of single atoms}

Following the adiabatic preparation and possible further manipulations (e.g., modulation of the lattice depth), the atoms were detected in situ using fluorescence imaging. A detailed account of the imaging technique can be found in Refs. [17, 74] and we only summarize the key points here.

The imaging was performed with a high-resolution objective, which was placed in 


\section{Overview of the experimental procedure}

front of the same vacuum window that was also used as a mirror for the vertical lattice axis (Fig. 3.1). The numerical aperture of the objective was $\mathrm{NA}=0.68$, yielding a spatial resolution of about $700 \mathrm{~nm}$ for an imaging wavelength of $780 \mathrm{~nm}$.

For the imaging, all lattice depths were increased to typically $3000 E_{r} \approx k_{B} \cdot 300 \mu \mathrm{K}$ on a time scale much faster than the many-body dynamics. As a result, the original density distribution of the gas was instantaneously frozen. The detailed sequence consisted of a two-step process, where the first step was an exponential ramp to $\approx 80 E_{r}$ with a duration of $0.2 \mathrm{~ms}$ that already froze the dynamcis (not shown in Fig.3.2). The second ramp to $\approx 3000 E_{r}$ is included to have sufficiently deep lattices for the imaging process. To image the frozen gas, we optically pumped the atoms from $5 S_{1 / 2}, F=1$ to $5 S_{1 / 2}, F=2$ and shone in imaging laser light with a red-detuning of $\approx 50 \mathrm{MHz}$ to the free-space resonance of the $5 S_{1 / 2}, F=2$ to $5 P_{3 / 2}$, $F=3$ transition. The scattered photons were observed through the high-resolution objective and an additional lens, which focused the light on an emccd camera.

Typical fluorescence images show individual atoms in the two-dimensional lattice (see Fig. 3.1c). Here we discuss a dilute, non-degenerate cloud and turn to dense, degenerate clouds in Ch.4. Approximately 5000 photons are detected per atom during an illumination time of about $1 \mathrm{~s}$, resulting in a high signal-to-noise ratio. To achieve this, we had to inhibit tunnelling (or even loss) of the atoms during the imaging phase. Such tunnelling processes can occur even in a very deep optical lattice of $3000 E_{r}$ if the atoms are thermally excited to higher bands as a result of the recoil heating by the imaging light. To suppress this thermally activated hopping, we laser-cooled the atoms by setting up the imaging beams in an optical molasses configuration, which resulted in sub-Doppler temperatures of $\approx 30 \mu \mathrm{K}$.

Note that the imaging process is destructive for the many-body state (see also Sec.3.5). To collect statistics for a given observable, the experiment had to be repeated in a loop starting with the preparation of the two-dimensional degenerate gas.

\subsection{Parity projection}

Instead of the actual density distribution, our images show the parity of the occupation number on each lattice site (Fig. 3.3). This is a consequence of a pairwise loss process due to light-assisted collisions [16, 17, 83], occurring on a time scale of typically $100 \mu s$, which is much faster than the illumination time of about $1 \mathrm{~s}$. Without additional steps, this loss would occur in the beginning of the illumination time, but some of the lost atoms would be recaptured due to the molasses cooling leading to an unwanted background signal. To avoid this, we trigger light-assisted collisions before repumping and before switching on the imaging light with a $50 \mathrm{~ms}$ pulse of light resonant for the $5 S_{1 / 2}, F=2$ to $5 P_{3 / 2}, F=3$ transition. At this stage in the 


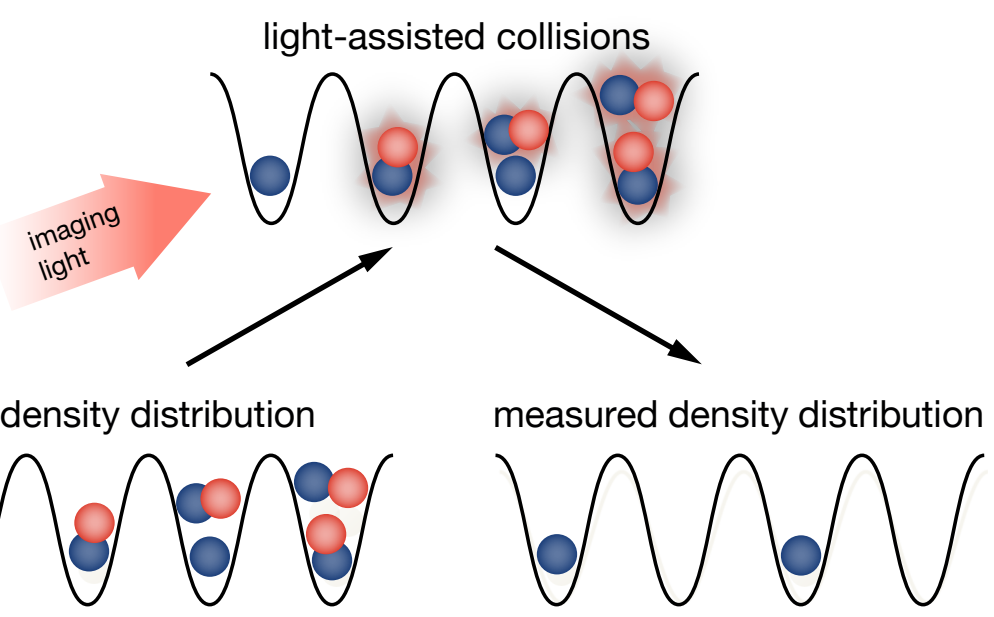

Figure 3.3.: Parity-projection mechanism. Illustration of the parity-projection mechanism. Due to light-assisted collisions, pairs of atoms are lost in the imaging process. As a consequence, the measured density distribution shows the parity of the initial on-site occupation number.

sequence, the atoms are however in the state $5 S_{1 / 2}, F=1$ and the light is $6.8 \mathrm{GHz}$ red detuned for the $5 S_{1 / 2}, F=1$ to $5 P_{3 / 2}, F=3$ transition, but efficiently excites atom pairs into molecular potentials leading to a rapid loss. As a result, doubly occupied lattice sites appear as empty sites in the images. Similarly, three atoms on a site are imaged as a single atom as two atoms undergo a pairwise collision. In general, we only observe a signal from sites with an odd occupation number and the measured occupation number can be written as the on-site parity $P_{i}=\bmod _{2} n_{i}$ in terms of the actual occupation number $n_{i}$, where $n_{i}$ and $P_{i}$ are eigenvalues of the on-site number operator $\hat{n}_{i}$ and the on-site parity operator $\hat{P}_{i}=\bmod _{2} \hat{n}_{i}$, respectively.

This might seem like a severe limitation, but the information of the parity is sufficient to study many interesting properties of the superfluid-Mott-insulator transition. This is because excitations in the Mott phase (e.g., with unity filling) typically appear as an empty or a doubly occupied site on a background with fixed mean density. In many situations, it is not necessary to distinguish both cases and the parity information is sufficient. Deep in the superfluid limit, however, the parity information is usually insufficient due to the large density fluctuations in this regime. Nevertheless, this can be circumvented in some situations by adiabatically connecting the system to the Mott-insulating phase (Part III). 


\section{Overview of the experimental procedure}

\subsection{Observable}

The described measurement technique detects more than the average on-site parity, but captures fluctuations and correlations in the system as well. An arbitrary manybody state $|\Psi\rangle$ at zero temperature can be written in terms of on-site Fock states (Ch. 2) as

$$
|\Psi\rangle=\sum_{\left\{n_{i}\right\}} C\left(n_{1}, \ldots, n_{N}\right)\left|n_{1}, \ldots, n_{N}\right\rangle
$$

where the sum runs over all possible configurations of on-site numbers $\left\{n_{i}\right\}=$ $\left(n_{1}, \ldots, n_{N}\right)$ and $i$ refers to a specific lattice site.

The freezing of the density distribution and the subsequent scattering of imaging light can be interpreted as a projective measurement. It leads to a projection onto a specific state $|\Psi\rangle_{\text {proj }}=\left|n_{1}, \ldots, n_{N}\right\rangle$ with a quantum mechanical probability $p\left(n_{1}, \ldots, n_{N}\right)=\left|C\left(n_{1}, \ldots, n_{N}\right)\right|^{2}$.

We first discuss the situation as it would be without parity projection. The crucial point is that the measurement would yield information about all occupation numbers $n_{i}$ of the projected state $|\Psi\rangle_{\text {proj }}=\left|n_{1}, \ldots, n_{N}\right\rangle$ in a single experimental run. Repeating the experiment from the start, we would observe the occupation numbers of a new $|\Psi\rangle_{\text {proj }}$ in each iteration. With this, we could gather an increasing amount of statistics and finally reconstruct the full joint probability distribution $p\left(n_{1}, \ldots, n_{N}\right)=$ $\left|C\left(n_{1}, \ldots, n_{N}\right)\right|^{2}$ to observe a specific set of occupation numbers $\left(n_{1}, \ldots, n_{N}\right)$ on all lattice sites. This includes more information than the average on-site density because from the knowledge of $p\left(n_{1}, \ldots, n_{N}\right)$, one could calculate all possible density-density correlation functions $\left\langle\prod_{i \in M} \hat{n}_{i}\right\rangle=\sum_{\left\{n_{i}\right\}} \prod_{i \in M} n_{i} p\left(n_{1}, \ldots, n_{N}\right)$, where the product runs over an arbitrarily chosen set of lattice sites $M$.

Including the parity projection mechanism, the most general observable is the joint probability distribution $p_{p}\left(P_{1}, \ldots, P_{N}\right)$ to observe a set of on-site parities $\left(P_{1}, \ldots, P_{N}\right)$ on all lattice sites. However, the full reconstruction of $p_{p}\left(P_{1}, \ldots, P_{N}\right)$ is a demanding task, since it requires a very large number of experimental repetitions. In practice, we evaluated various correlation functions using an additional spatial average in order to improve the statistical noise on our data.

The detection technique can not directly distinguish the pure state $|\Psi\rangle=$ $\sum_{\left\{n_{i}\right\}} C\left(n_{1}, \ldots, n_{N}\right)\left|n_{1}, \ldots, n_{N}\right\rangle$, from a mixed state, described by the density operator

$$
\hat{\rho}=\sum_{\left\{n_{i}\right\}}\left|C\left(n_{1}, \ldots, n_{N}\right)\right|^{2} \hat{P}_{\left|n_{1}, \ldots, n_{N}\right\rangle},
$$

with the projection operator $\hat{P}_{\left|n_{1}, \ldots, n_{N}\right\rangle}=\left|n_{1}, \ldots, n_{N}\right\rangle\left\langle n_{1}, \ldots, n_{N}\right|$. This is the case because both of these states yield the same joint probability distributions $p\left(n_{1}, \ldots, n_{N}\right)=$ 
$\left|C\left(n_{1}, \ldots, n_{N}\right)\right|^{2}$ and $p_{p}\left(P_{1}, \ldots, P_{N}\right)$. Therefore, the imaging technique, with or without parity projection, cannot detect off-diagonal elements (i.e., coherences) of density operators written in an on-site number basis. 
3. Overview of the experimental procedure 


\section{Single-site-resolved imaging and thermometry of atomic limit Mott insulators}

In this chapter, we apply the imaging technique described in Ch. 3 to the deep Mott insulating limit at $J / U \approx 0$ (Ch.2.2). This limit is an ideal testbed for imaging of dense clouds since the many-body state including finite temperature and the harmonic confinement can be analytically found.

\subsection{Theory for the ground state and the influence of a trapping potential}

We will consider the regime in which hopping can be neglected compared to interactions $(J / U \approx 0)$. The atomic limit is particularly easy to understand because the Bose-Hubbard Hamiltonian $\hat{H}_{\mathrm{BH}}$ can be written as a sum of on-site terms $\hat{H}_{i}$

$$
\hat{H}_{\mathrm{BH}} \approx \sum_{i} \hat{H}_{i} \equiv \sum_{i}\left[\frac{U}{2} \hat{n}_{i}\left(\hat{n}_{i}-1\right)-\mu \hat{n}_{i}\right] .
$$

In this approximation, the interplay between the interaction term and the chemical potential term defines the local state of the system.

Starting from Eq.4.1, we will first find the energy-eigenspectrum of the system. Based on this, we will discuss some ground-state properties, the role of the harmonic trapping potential and the influence of finite temperature.

\subsubsection{Eigenspectrum and eigenstates}

In the atomic limit, we can easily find all eigenstates of the system. They are products of on-site wave functions since the Hamiltonian is a sum of on-site terms. These onsite wave functions are Fock-states $\left|n_{i}\right\rangle$ with a well defined occupation number $n_{i}$, because the on-site terms $H_{i}$ are diagonal in the number operator $\hat{n}_{i}$. The eigenstates $|\Psi\rangle$ can therefore be written as $|\Psi\rangle=\prod_{i}\left|n_{i}\right\rangle$. We can define an on-site eigenenergy $E\left(n_{i}\right)$ by $\hat{H}_{i}\left|n_{i}\right\rangle=E\left(n_{i}\right)\left|n_{i}\right\rangle$ and find from Eq. 4.1 that

$$
E\left(n_{i}\right)=\frac{U}{2} n_{i}\left(n_{i}-1\right)-\mu n_{i}
$$

With this, we can write the (total) eigenenergy $E$ as $E=\sum_{i} E\left(n_{i}\right)$.

It is instructive to plot Eq. 4.2 for various values of $n_{i}$, which is shown in Fig. 4.1. We 


\section{Single-site-resolved imaging and thermometry of atomic limit Mott insulators}
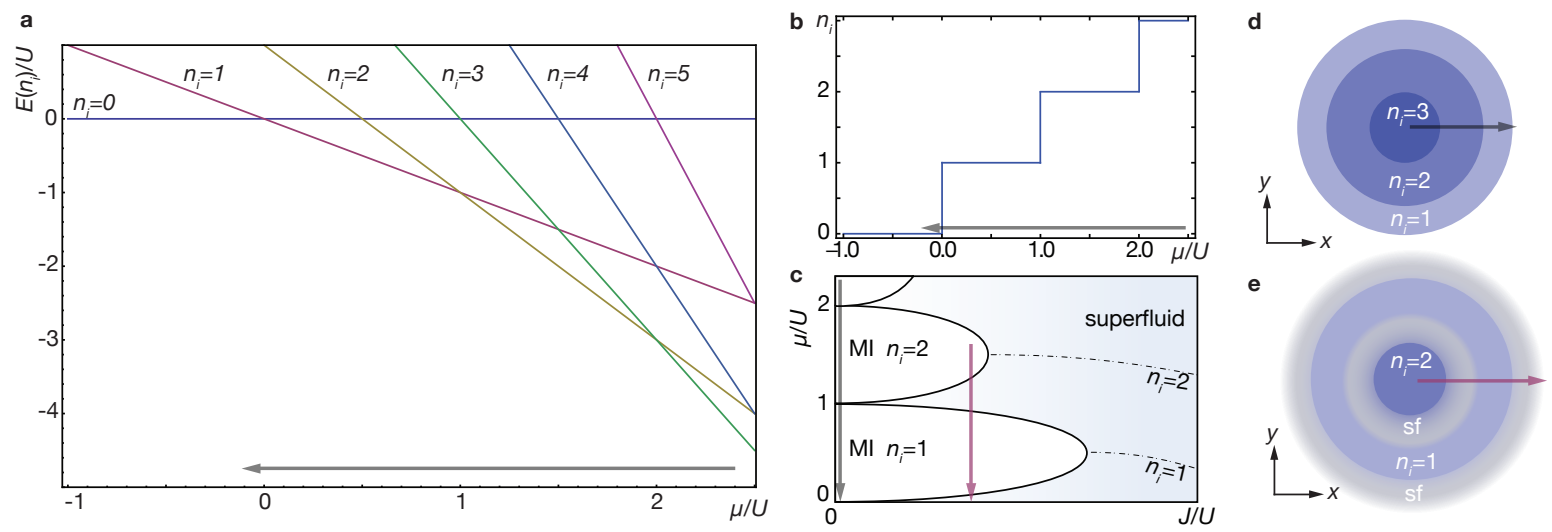

Figure 4.1.: Eigenenergies and on-site occupation in the atomic limit. a, Plot of the eigenenergies $E\left(n_{i}\right)=\frac{U}{2} n_{i}\left(n_{i}-1\right)-\mu n_{i}$ of the Bose-Hubbard model in the atomic limit (Eq. 4.2). Lines show $E\left(n_{i}\right)$ as a function of the chemical potential $\mu$ for different values of the on-site occupation number $n_{i}=0-5$. Each graph is labeled with the corresponding $n_{i}$. For $\mu / U<0$, the eigenstate with the lowest energy (i.e., the ground state) is given by $n_{i}=0$, while for $0<\mu / U<1$, the ground state is given by $n_{i}=1$ and so on. $\mathbf{b}$, Groundstate occupation number as a function of $\mu$. $\mathbf{c}$, The graphs in $\mathbf{a}$ and $\mathbf{b}$ can be considered as a cut in the Bose-Hubbard phase diagram along the $\mu$-direction at constant $J / U=0$, indicated by the grey-shaded arrow. Each step for $n_{i}$ in $\mathbf{b}$ corresponds to a different Mott lobe. $\mathbf{d}$, Sketch of the in-trap density for a two-dimensional geometry. In a trapped system with potential $V_{i}$, the local chemical potential $\mu_{i}=\mu-V_{i}$ decreases, going outwards from the center of the trap. In a two-dimensional, radially symmetric geometry, we therefore find a series of Mott-insulating rings, reminiscent of a wedding cake. e, For $J / U>0$, indicated by the red arrow in $\mathbf{c}$, the locally varying potential can lead to an alternating series of Mott-insulating and superfluid rings.

find that for a given value of $\mu$ the energy is minimized (i.e., the ground state is found) by choosing $n_{i}$ to be

$$
n_{i}=\lceil\mu / U\rceil,
$$

where $\lceil x\rceil$ is the ceiling of $x$. This corresponds to a series of steps for $n_{i}$ as a function of $\mu / U$ (Fig. 4.1b).

As a consequence, we find that $\partial\left\langle\hat{n}_{i}\right\rangle / \partial \mu=0$, i.e. the system is incompressible, except for the singular points for which $\mu / U$ equals a non-negative integer. Since the ground state is a simple product of Fock-states, we also find that density fluctuations are completely suppressed (i.e., $\left\langle\hat{n}_{i}^{2}\right\rangle-\left\langle\hat{n}_{i}\right\rangle^{2}=0$ ). 


\subsubsection{Influence of the trapping potential}

In a trapped system like ours, in addition to the three terms already present in the original Bose-Hubbard model (Sec. 2.4), the potential energy due to the external harmonic confinement must also be considered. With Eq. 2.26, we can write the BoseHubbard model in the atomic limit including harmonic confinement as

$$
\hat{H}_{\mathrm{BH}} \approx \sum_{i} \hat{H}_{i} \equiv \sum_{i}\left[\frac{U}{2} \hat{n}_{i}\left(\hat{n}_{i}-1\right)-\mu_{i} \hat{n}_{i}\right] .
$$

Therefore, the chemical potential $\mu$ in the formulas for the eigenergies Eq.4.2 and the groundstate occupation number Eq. 4.3 is simply replaced by a local chemical potential $\mu_{i}$ given by

$$
\begin{aligned}
& \mu_{i}=\mu-V_{i} \\
& V_{i}=\frac{1}{2} m\left(\omega_{x}^{2} x_{i}^{2}+\omega_{y}^{2} y_{i}^{2}\right),
\end{aligned}
$$

where $V_{i}$ is the potential energy in a harmonically approximated trap. In contrast to Eq. 2.27, we only consider harmonic confinement in the $x$ and $y$-directions, since our system is effectively two-dimensional (Sec. 3.1). To distinguish between $\mu_{i}$ and $\mu$, we will refer to $\mu$ as the global chemical potential.

For a given global chemical potential, the local chemical potential $\mu_{i}$ decreases as a function of distance from the center of the trap. We therefore find the maximal $\mu_{i}$ and consequently the highest occupation number in the trap center. Going outwards, we find steps in the occupation number, whenever $\mu_{i}$ equals a multiple of $U$. In a twodimensional, radially symmetric situation, this leads to a series of Mott-insulating rings with decreasing occupation number (Fig. 4.1.1 c). The outer edge of the cloud is given by the point for which the local chemical potential $\mu_{i}$ vanishes, yielding a cloud radius of $\sqrt{\mu /(1 / 2) m \omega_{\alpha}^{2}}$ in $\alpha=x, y$ direction. 


\subsection{Single-site-resolved fluorescence images}

Experimentally, we can reach the atomic limit by increasing the lattice depth of both horizontal lattices adiabatically (Sec. 3.2) until tunneling between neighboring sites is completely suppressed (Fig. 2.4c). For the data presented in this chapter, we used a final lattice depth of 23(1) $E_{r}$ yielding $J / U \approx 3 \cdot 10^{-3}$, which is about a factor of 20 smaller than the critical value $(J / U)_{c} \approx 0.06$ for the superfluid-Mott-insulator transition in $2 \mathrm{~d}$ (Table 2.1). The data was therefore taken in the deep atomic limit. The duration of the s-shaped ramp for the horizontal lattice axes was $75 \mathrm{~ms}$ and the final lattice depth was the same for both axes.

After this preparation, we took in situ fluorescence images of the system (Sec. 3.3) for a range of different atom numbers. The raw data of these images are shown in Fig. 4.2. As the atom number increases, we observe ring-like structures, which we attribute to the presence of Mott-insulating rings. The rings have an increasing occupation number towards the center of the trap, but due to the parity projection mechanism, these appear as rings with an occupation number that alternates between one and zero (Sec.3.4). In contrast to the prediction for the ground-state, the images show a small number of density fluctuations, which we attribute to thermal excitations in the system (Sec. 4.3 contains a thorough analysis of this effect).

In contrast to the image of a dilute thermal cloud shown in Fig. 3.1, single atoms are not visible in the dense regions of the Mott-insulating rings in Fig. 4.2. However, the signal-to-noise ratio and the spatial resolution of our imaging procedure are sufficient to reconstruct the atom distribution in the lattice using a computer algorithm (Fig. 4.3). The output of the algorithm is digital information in matrix-form containing the parity of the on-site occupation number for each lattice-site. Details of this reconstruction procedure are given in Refs. [17, 74]. 

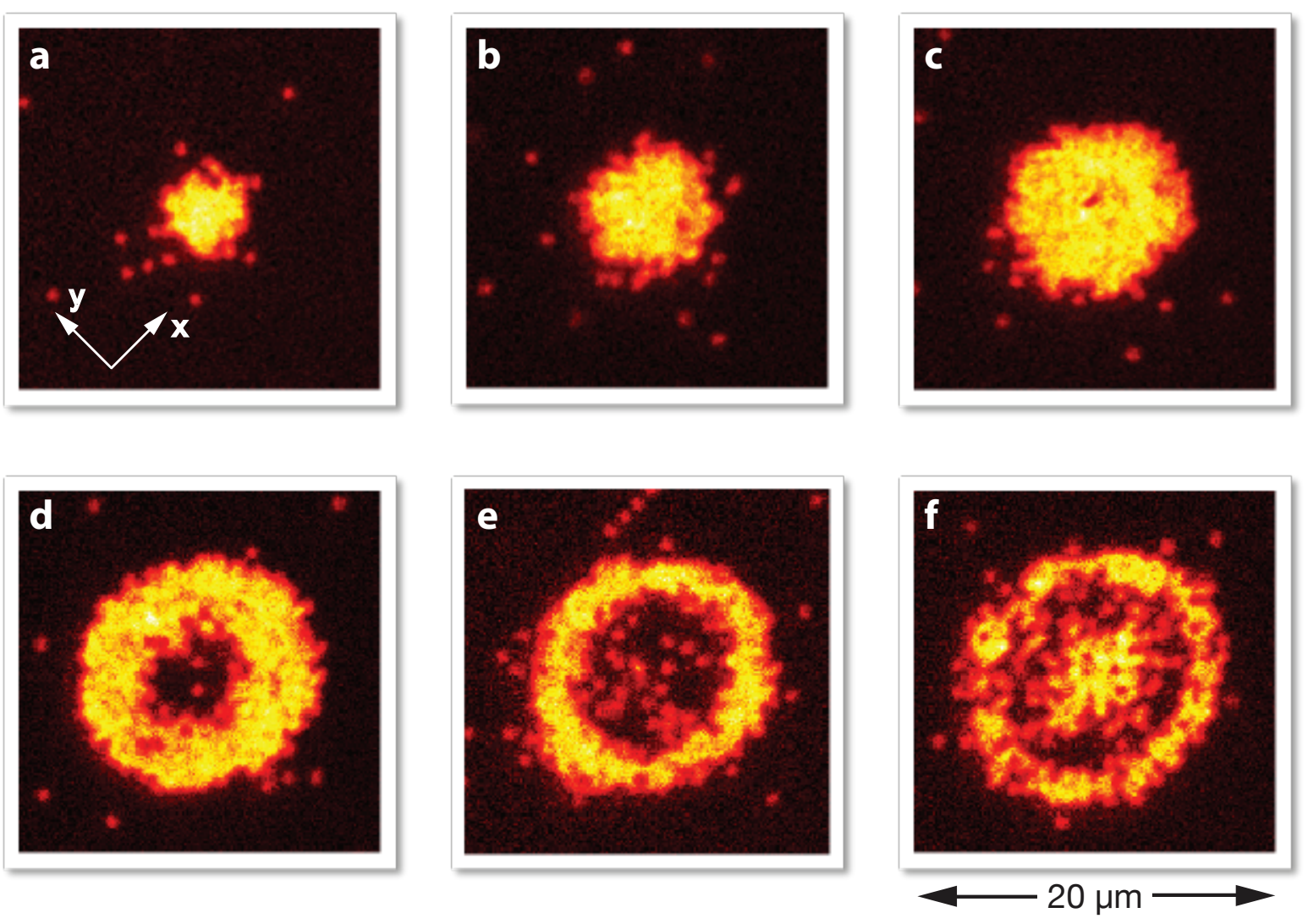

Figure 4.2.: Images of atomic limit Mott insulators for an increasing atom number. Raw data for images of Mott insulators in the atomic limit. The atom number increases from a to f. The emergence of a ring-like structure is clearly visible, which is attributed to the presence of Mott-insulating rings. The influence of the parity projection (Sec. 3.4) is seen for larger atom numbers, e.g., the hole in the center of $\mathbf{d}$ and $\mathbf{e}$ is interpreted as the image of a ring with doubly occupied sites that appear empty after parity projection. The picture in $\mathbf{f}$ shows a density distribution similar to the sketch in Fig. 4.1d, with a central region with three atoms on each site. The horizontal lattice axes are approximately along the diagonal of the images (see coordinate system in a.). 


\section{Single-site-resolved imaging and thermometry of atomic limit Mott insulators}
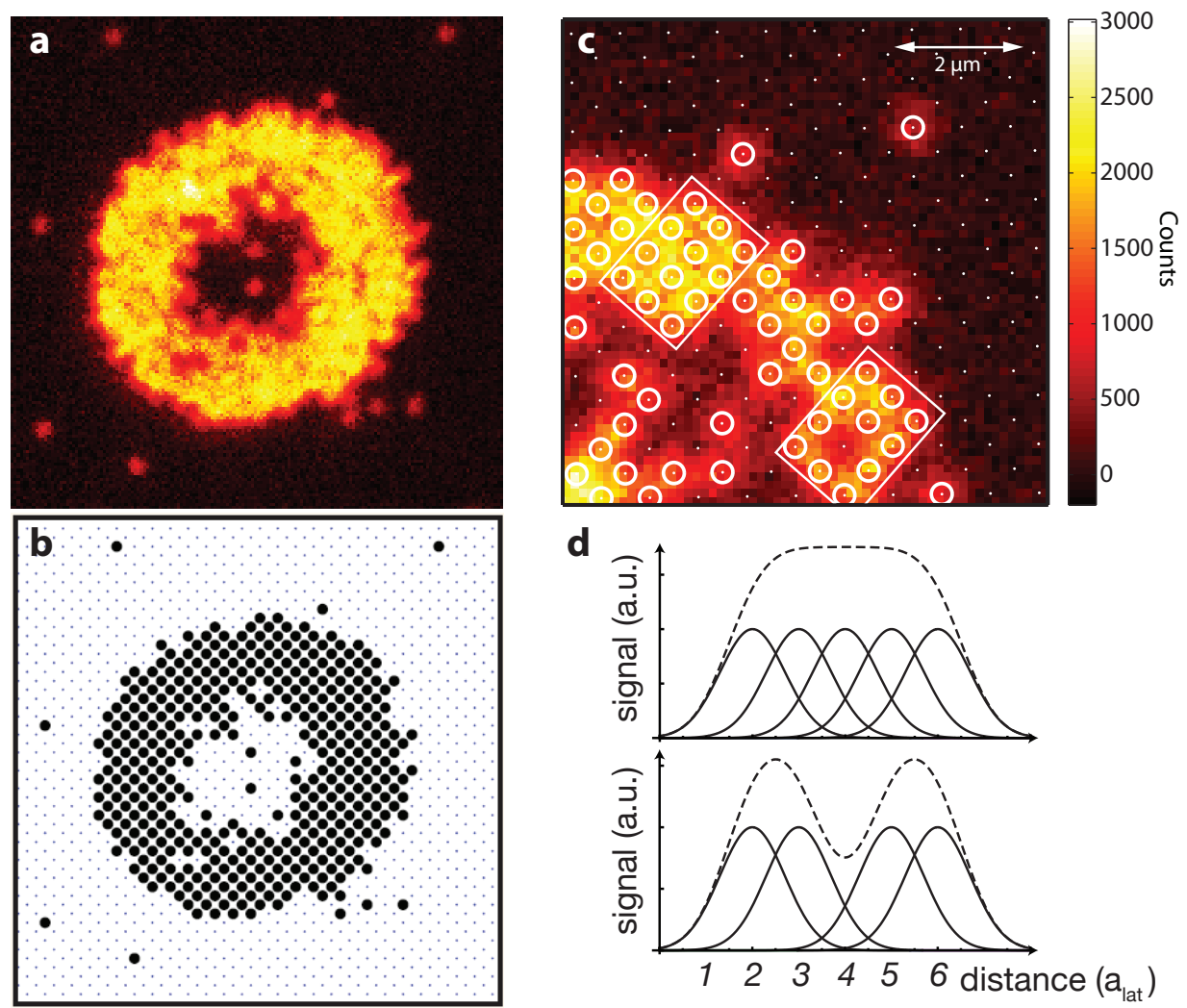

Figure 4.3.: Single-site reconstruction. a, Same data as Fig. 4.2d. b, Reconstructed single-siteresolved information of the on-site parity for the data in a showing large areas with a high probability to find a given odd or even occupation number on a lattice-site. The low-defect density in these regions is in accordance with the theoretical prediction for the atomic limit, where the on-site wave function is given by a Fock state with a well defined on-site occupation number. Remaining number fluctuations are attributed to thermal excitations (Sec. 4.3). c, Zoom into the upper right part of Fig. 4.2f. Open circles mark the positions of single atoms as found by the reconstruction algorithm. $\mathbf{d}$, The reconstruction is possible, even if the optical resolution (about 700nm) is slightly larger than the lattice spacing $\left(a_{\text {lat }}=532 \mathrm{~nm}\right)$. Five atoms sitting on neighboring lattice sites are sketched with their point spread functions (solid line, top panel). The sum of the signals (dashed line) shows only a broad feature. However, if one of the atoms is missing (lower panel) a dip in this feature appears. The reconstruction algorithm can therefore still distinguish both situations (compare, e.g., the two boxed areas in c). 


\subsection{Influence of finite temperature and in situ thermometry}

The experimentally observed images of Mott insulators (Fig. 4.2 and 4.3) show density fluctuations that cannot be described by the theory for atomic limit Mott insulators in their ground state (Sec.4.1.1). This indicates that the finite temperature of the sample resulted in thermal excitations of the system which appeared as defects in the otherwise fluctuation-free Mott rings. To treat such a situation, we will present an extension of Sec. 4.1.1 that includes the thermally activated occupation of excited states [84].

In the atomic limit, the system can be treated locally for each individual lattice-site, since the hopping term in the Bose-Hubbard Hamiltonian can be neglected (Eq. 2.1). For a given site $i$, we found the on-site energy $E\left(n_{i}\right)=\frac{U}{2} n_{i}\left(n_{i}-1\right)-\mu n_{i}$ for an occupation number $n_{i}$ (Eq. 4.2). We can now imagine that this lattice site is in thermal contact with the remainder of the system. However, the rate of energy exchange between the site and the rest of the system should be small enough for the atomic limit approximation to hold. In order to ensure thermal equilibrium in this situation, the system needs to relax for a long time.

Assuming thermal equilibrium, we can assign a temperature $T$ to the system, which must be the same at a given lattice site $i$ and in the remainder of the system. The probability $p\left(n_{i}\right)$ to find a state with occupation number $n_{i}$ (with energy $E\left(n_{i}\right)$ ) on lattice site $i$, is then given by the Boltzman factor

$$
p\left(n_{i}\right)=\frac{\exp \left(-E\left(n_{i}\right) / k_{B} T\right)}{Z}
$$

where $Z=\sum_{n_{i}} \exp \left(-E\left(n_{i}\right) / k_{B} T\right)$ is the partition function. This result can also be formally derived as the zeroth-order term in a high-temperature expansion [85].

With Eq. 4.8 at hand, we can calculate various on-site quantities. For example, the average on-site density $\left\langle\hat{n}_{i}\right\rangle$ is given by $\left\langle\hat{n}_{i}\right\rangle=\sum_{n_{i}} n_{i} p\left(n_{i}\right)$. In the experiment, we expect to measure the on-site parity $\hat{P}_{i}$, for which the average value can be easily computed as

$$
\left\langle\hat{P}_{i}\right\rangle=\sum_{n_{i}} \bmod _{2}\left(n_{i}\right) p\left(n_{i}\right)
$$

with the on-site parity operator $\hat{P}_{i}$ given by $\hat{P}_{i}\left|n_{i}\right\rangle=\bmod _{2}\left(n_{i}\right)\left|n_{i}\right\rangle$.

The influence of temperature in the atomic limit can be readily understood by examining the on-site eigenenergies $E\left(n_{i}\right)$ (Eq. 4.2 and Fig. 4.1). In Fig. 4.4a, we show the difference between the eigenenergies $E\left(n_{i}\right)$ and the groundstate energy $E_{0}$. We focus on the first Mott lobe (shaded area) with an occupation number $n_{i}=1$ in its ground state. For $\mu_{i} / U<0.5$, the first excited state has $n_{i}=0$, while for $\mu_{i} / U>0.5$ 


\section{Single-site-resolved imaging and thermometry of atomic limit Mott insulators}
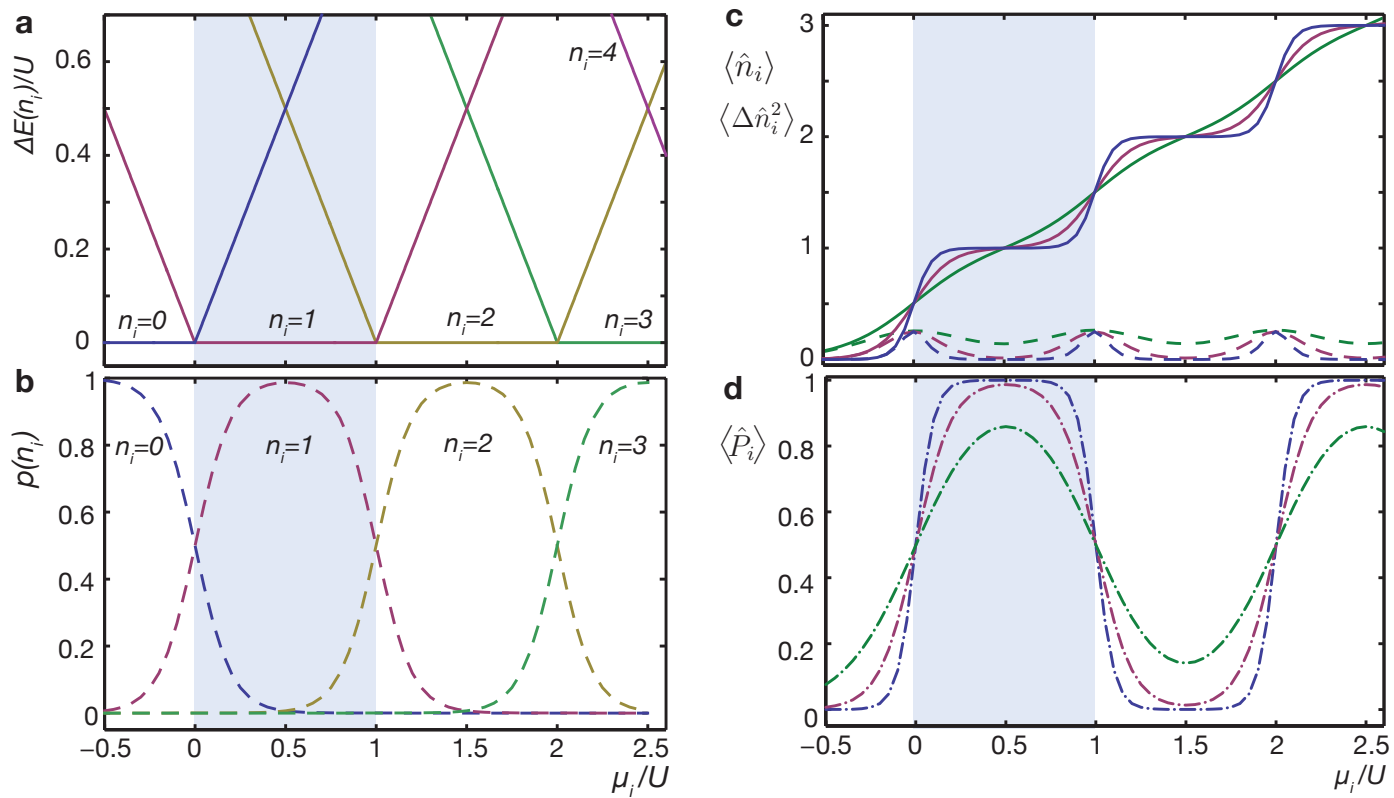

Figure 4.4.: Influence of temperature in the atomic limit. a, Difference between the eigenenergies $E\left(n_{i}\right)$ and the groundstate energy $E_{0}$, defined as $\Delta E\left(n_{i}\right)=E\left(n_{i}\right)-E_{0}$. b. Probability $p\left(n_{i}\right)$ (Eq. 4.8) to find a state with $n_{i}$ for a temperature of $k_{B} T=0.1 U$. c, Average density $\left\langle\hat{n}_{i}\right\rangle=\sum_{n_{i}} n_{i} p\left(n_{i}\right)$ (solid lines) and density fluctuations $\left\langle\Delta \hat{n}_{i}^{2}\right\rangle=\left\langle\hat{n}_{i}^{2}\right\rangle-\left\langle\hat{n}_{i}\right\rangle^{2}$ (dashed lines) for three different temperatures $k_{B} T=0.05 U$ (blue), $k_{B} T=0.1 U$ (purple), $k_{B} T=0.2 U$ (green). d, Average parity $\left\langle\hat{P}_{i}\right\rangle=\sum_{n_{i}} \bmod _{2}\left(n_{i}\right) p\left(n_{i}\right)$ for the same temperatures (dashed dotted lines).

it has $n_{i}=2$. For $\mu_{i}=0.5$, the gap to the first excited state is maximal and we therefore expect thermally induced density fluctuations to be minimal. In contrast, at the edges of the lobe (e.i., for $\mu_{i} / U=0$ and $\mu_{i} / U=1$ ), the excitation gap closes and density fluctuations are easily excited. This has direct influence on the probability to observe a certain on-site occupation number (Fig. 4.4b). In the center of the first Mott lobe (around $\mu_{i} / U=0.5$ ), we find a high probability for the groundstate $\left(n_{i}=1\right)$. At the edges of the lobe, the probability to observe excited states (with $n_{i}=0$ or 2 ) is strongly increased. As a consequence, the step-like density distribution of the $T=0$ system becomes washed out with higher temperatures (Fig. 4.4c), while density fluctuations increase, in particular at the transition regions between the Mott lobes (e.i., at $\mu_{i} / U=0,1,2$ ). Also, the alternating step-structure of the average on-site parity is smoothed with increasing temperature (Fig. $4.4 \mathrm{~d}$ ). The average parity yields direct information on the probabilities $p\left(n_{i}\right)$, if the temperature is low enough to consider only the lowest lying excited states. In the region with $\mu_{i} / U \lesssim 1$, e.g., the average parity equals the probability to find a single atom on a lattice site $p\left(n_{i}=1\right)$, as the relevant excited states (with $n_{i}=0$ or 2 ) have an occupation number with 
odd parity: $\left\langle\hat{P}_{i}\right\rangle=\sum_{n_{i}} \bmod _{2}\left(n_{i}\right) p\left(n_{i}\right) \approx p\left(n_{i}=1\right)$ (compare, dashed purple line in Fig. $4.4 \mathrm{~b}$ and dashed dotted purple line in Fig. $4.4 \mathrm{~d}$ for $\mu_{i} / U<1$ ).

Based on these findings, we can formulate a fitting function for the observed signal in the atomic limit. In Sec. 4.1.2, we found that for a harmonically trapped system, the global chemical potential $\mu$ is replaced by a local chemical potential $\mu_{i}=\mu-V_{i}$, which yields $E\left(n_{i}\right)=\frac{U}{2} n_{i}\left(n_{i}-1\right)-\left(\mu-V_{i}\right) n_{i}$ for the on-site energies. With this, we can find a fitting function by replacing the on-site energies in Eq.4.8 with the previous formula and inserting $p\left(n_{i}\right)$ in Eq. 4.10. This yields

$$
\left\langle\hat{P}_{i}\right\rangle=\sum_{n_{i}} \bmod _{2}\left(n_{i}\right) \frac{\exp \left(-\frac{1}{k_{B} T}\left(\frac{U}{2} n_{i}\left(n_{i}-1\right)-\left(\mu-V_{i}\right) n_{i}\right)\right)}{Z},
$$

with $Z=\sum_{n_{i}} \exp \left(-\frac{1}{k_{B} T}\left(\frac{U}{2} n_{i}\left(n_{i}-1\right)-\left(\mu-V_{i}\right) n_{i}\right)\right)$ and fitting parameters $T / U, \mu / U$ and $\omega_{\alpha}^{2} / U$. For details concerning the fitting procedure and the treatment of the slight ellipticity of the clouds, see Refs. [17, 74].

In Fig. 4.5, we show the results of such a fit. The main outcome of this procedure is the determination of the temperature $T$ in units of the interaction energy $U$. Importantly, we assumed a constant temperature across the whole sample. Therefore, the very good agreement of the fit with the data suggests that the gas was sufficiently thermalized.

Additionally, we stress that the described thermometry scheme is capable of extracting the temperature from a single experimental image. This can be understood as follows. As argued above, each lattice site can be viewed as its own physical system which is in thermal equilibrium with the remainder of the system. The on-site physics is, thus, described by a grand canonical ensemble with a temperature $T$ and a local chemical potential $\mu_{i}$. For each lattice site with the same distance to the center of the trap (ignoring possibly different $\omega_{\alpha}$ in $\alpha=x, y$ directions), we find the same local chemical potential. We therefore have several 'copies' of the same statistical ensemble on a ring. The azimuthal average shown in Fig. 4.5 yields a statistical average of these systems in a single shot. The data points in Fig. 4.5b-e can therefore be considered as ensemble averages at a given local chemical potential. We then find the temperature that best matches these ensembles by fitting Eq. 4.10.

This thermometry scheme describes a novel method for a precise temperature measurement in the atomic limit $(J / U \approx 0)$. An extension for a finite, but small coupling between lattice sites might be possible using a second-order high-temperature expansion [85]. However, recent Quantum Monte Carlo simulations suggest that the temperature in units of the interaction strength $U$ is approximately constant for $J / U \lesssim(J / U)_{\mathcal{C}}$ (App.B). We can therefore approximate the temperature in units of $U$, even at a non-zero $J$, using an adiabatic ramp to the atomic limit. 

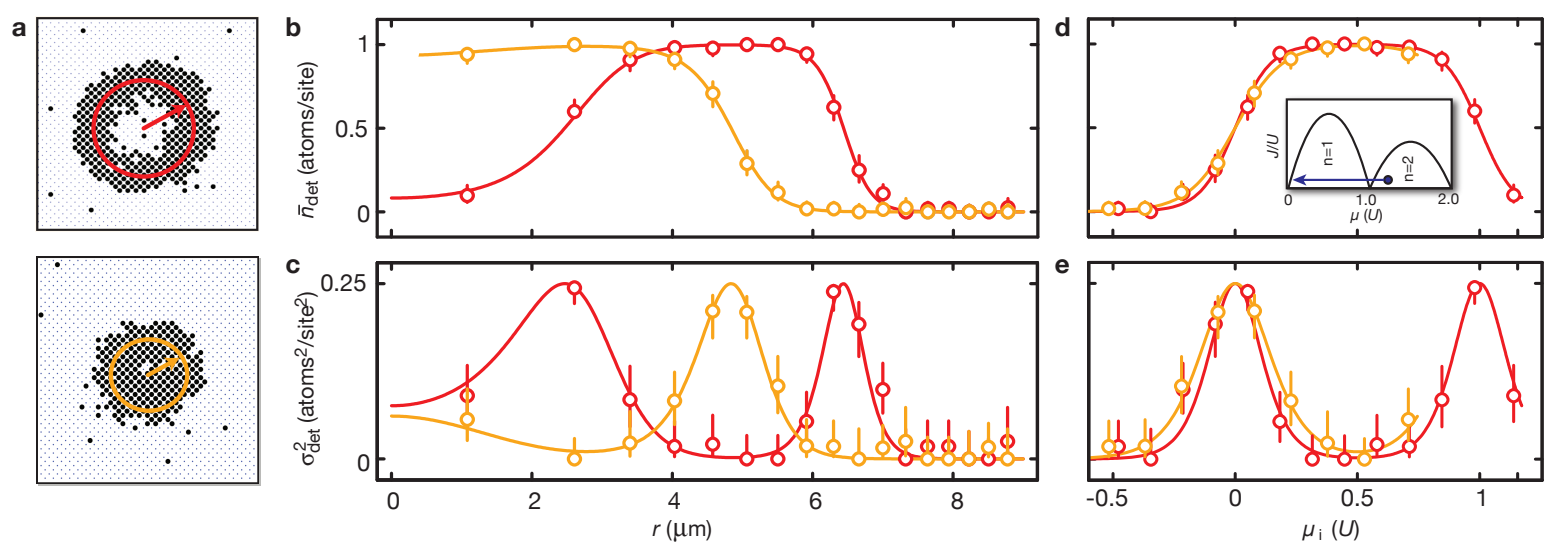

Figure 4.5.: In situ thermometry. a, Top panel: reconstruction of raw data from Fig. 4.2d (red data in b-e). Bottom panel: reconstruction of raw data from Fig. 4.2c (yellow data in $\mathbf{b}$-e). The data shown in $\mathbf{b}$-e stem from an azimuthal average of the reconstructed images, illustrated by the circles and arrows in $\mathbf{a}$. $\mathbf{b}$, Detected occupation number $\bar{n}_{\text {det }}$ as a function of the radial distance from the center $r$ (open circles). Solid lines show a fit with the expectation for the on-site parity $\left\langle\hat{P}_{i}\right\rangle=\sum_{n_{i}} \bmod _{2}\left(n_{i}\right) p\left(n_{i}\right)$, according to Eq. 4.10. The fitting parameters were $T / U, \mu / U$ and $\omega_{\alpha}^{2} / U$. We found temperatures of $k_{B} T / U=0.090(5)$ (yellow data) and $k_{B} T / U=0.074(5)$ (red data). c, Detected fluctuation of the occupation number $\sigma_{\operatorname{det}}^{2}$ (circles). Solid lines show a fit with the expectation for the variance of the on-site parity $\left\langle\Delta \hat{P}_{i}^{2}\right\rangle=\left\langle\hat{P}_{i}^{2}\right\rangle-\left\langle\hat{P}_{i}\right\rangle^{2}$. The good agreement in $\mathbf{b}$ and $\mathbf{c}$ shows that the measured atom number $\bar{n}_{\text {det }}$ indeed yields the on-site parity $\left\langle\hat{P}_{i}\right\rangle$. $\mathbf{d}$, and $\mathbf{e}$, Same as in b and c, but as a function of the local chemical potential $\mu_{i}$. Both graphs can be considered as an experimentally measured cut through the phase diagram at $J / U=0$ (inset in d). Errorbars denote Clopper-Pearson $68 \%$ confidence intervals. 


\subsection{Summary and conclusion}

We presented an experimental technique capable of single-site- and single-atomresolved detection of ultracold atoms in optical lattices using high-resolution fluorescence imaging [15-17]. More specifically, we were able to measure the on-site parity of an ultracold bosonic lattice-gas in a parameter regime, for which an accurate description in terms of the Bose-Hubbard Hamiltonian is possible (Ch. 2 and 3).

We showed that a reliable single-site-resolved detection is feasible even in dense, atomic limit Mott insulators (Sec. 4.2). In this limit, analytic expressions for the onsite occupation number are available that take into account a trapping potential and non-zero temperature. Based on this, we developed a novel thermometry scheme (Sec.4.3) that yields a precise temperature measurement in a single experimental run. The scheme enables the detection of temperature differences on the order of $k_{B} T / U \approx 0.01$, which, in combination with lattice modulation, forms a new precise tool for the spectroscopy of many-body systems (Part III).

It should be stressed that our technique is single-atom resolving in a single experimental run. As a consequence, it is possible to measure joint probability distributions that capture the correlations between fluctuations in different parts of the system (Sec.3.5). We made use of this capability to detect high-order parity-parity correlation functions in addition to the average on-site parity (Part II). 
4. Single-site-resolved imaging and thermometry of atomic limit Mott insulators 


\section{Part II.}

\section{Single-site- and single-atom-resolved detection of correlation functions}


Correlation functions play an important role for the theoretical and experimental characterization of many-body systems. In solid-state systems, they are usually determined through scattering experiments, whereas, in cold-gases systems, time-of-flight and in situ absorption imaging are the standard techniques. However, none of these methods allow for the in situ detection of spatially-resolved correlation functions at the single-particle level. Here we show the detection of correlation functions using in situ fluorescence imaging of ultracold bosonic atoms in an optical lattice (Part I). This method yields single-site- and single-atom-resolved images of the lattice gas in a single experimental run, thus gaining direct access to fluctuations in the many-body system. As a consequence, the detection of correlation functions between an arbitrary set of lattice sites is possible. This allows not only for the detection of two-point correlation functions (Ch.5) but also for the evaluation of non-local correlations (Ch. 6), which originate from of an extended region of the system and are used for the characterization of one-dimensional quantum phases that do not posses (quasi)-long-range order in the traditional sense. Furthermore, we give an interpretation of these findings in terms of dual transformations that also extends to the two-dimensional case (Ch. 7) where non-local correlation functions correspond to Wilson loop-type observables in the dual theory. 


\section{Detection of particle-hole pairs using two-site correlation functions}

Particle-hole pairs fundamentally determine the properties of the Mott insulator at finite-tunneling, such as its residual phase coherence [86], and lie at the heart of superexchange-mediated spin interactions that form the basis of quantum magnetism in multi-component quantum gas mixtures [28, 87-90].

We directly detect these pairs using single-site-resolved detection of two-site correlation functions across the superfluid-Mott-insulator transition (Fig. 5.1a). The results are compared to ab initio numerical calculations. Furthermore, our findings directly show the influence of the dimensionality of the system on the properties of the phase transition (Sec. 5.2 and 5.3).

\subsection{Introduction to particle-hole pairs and two-site correlation functions}

In contrast to Mott insulators in the atomic limit, Mott insulators at finite $J / U>0$ show number fluctuations even at zero temperature and are not described by simple product states. For small $J / U \ll(J / U)_{c}$, these fluctuations appear in the form of correlated particle-hole pairs formed by an extra particle and missing particle on two nearby sites. In the following, we deal with a situation where the region of interest lies within the $n_{i}=1$ Mott-insulating lobe and where the local zero-temperature state, in the atomic limit, is described by $|\Psi\rangle_{J / U=0}=\prod_{i}\left|n_{i}=1\right\rangle$. The emergence of quantum-correlated particle-hole pairs can then be readily understood within firstorder perturbation theory considering the tunneling term as a perturbation. To first order, one obtains for the ground state

$$
|\Psi\rangle_{J / U \ll(J / U)_{c}} \propto|\Psi\rangle_{J / U=0}+\frac{J}{U} \sum_{\langle i, j\rangle} \hat{b}_{i}^{\dagger} \hat{b}_{j}|\Psi\rangle_{J / U=0}
$$

The second term yields contributions of the schematic form $\hat{b}_{i}^{+} \hat{b}_{j}|\Psi\rangle_{J / U=0}=$ $\sqrt{2}|1,1, \ldots, 0,2, \ldots, 1,1\rangle$, with a sum over all positions and orientations of the particlehole pair formed by the neighboring empty- and doubly-occupied site. The state $|\Psi\rangle_{J / U \ll(J / U)_{c}}$ yields a probability to find a particle-hole pair on neighboring sites proportional to $(J / U)^{2}$ (Fig. 5.1b).

Closer to the transition to the superfluid phase, higher-order perturbation terms 


\section{Detection of particle-hole pairs using two-site correlation functions}

a

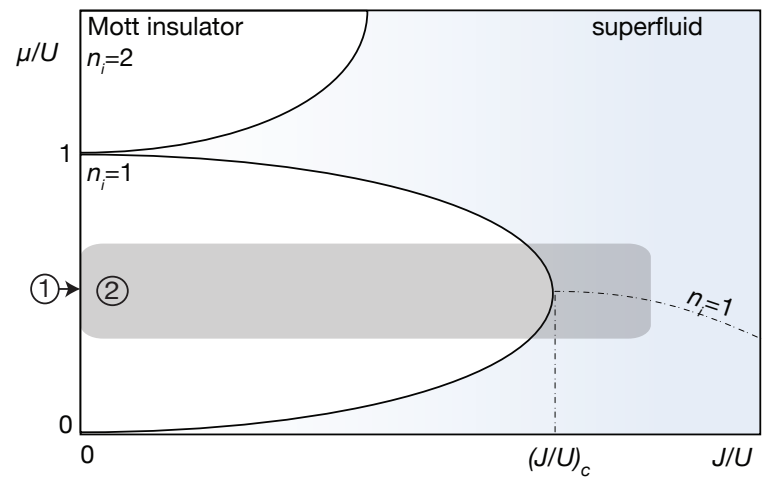

b

(1) atomic limit $(J / U=0)$

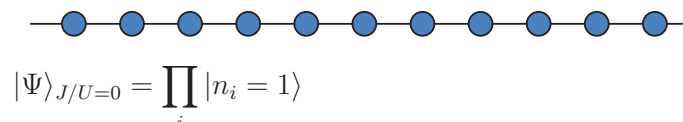

(2) small tunneling $\left(0<J / U<<(J / U)_{C}\right)$

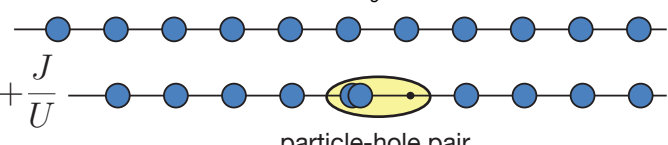

particle-hole pair

$|\Psi\rangle_{J / U \ll(J / U)_{c}} \propto|\Psi\rangle_{J / U=0}+\frac{J}{U} \sum_{\langle i, j\rangle} \hat{b}_{i}^{\dagger} \hat{b}_{j}|\Psi\rangle_{J / U=0}$

Figure 5.1.: Introduction to particle-hole pairs. a, Bose-Hubbard phase diagram. The current chapter deals with the single-site-resolved measurement of two-site correlation functions in a region of the phase diagram roughly given by the grey shaded area. $\mathbf{b}$, Upper panel: Illustration of the quantum state in the atomic limit $(J / U=0)$ labeled by corresponding number in a. We find a product wavefunction where every site is occupied by a single atom. Lower panel: Quantum state for a finite but small tunneling amplitude $\left(0<J / U \ll(J / U)_{c}\right)$ in first order perturbation in $J / U$. We find a superposition of the atomic limit ground state and states where a particle has hopped one lattice site. This leads to particle-hole pairs consisting of pairs of sites with an extra particle and a missing particle.

become more important. Intuitively, this leads to a rapid increase of bound particlehole pairs and an extension of their size, eventually resulting in deconfinement of the pairs at the transition point. One can view this process of proliferation and extension of particle-hole pairs as the driving force for the superfluid-Mott-insulator transition. However, more complicated clusters of particles might also play an important role. Additionally, the appearance of fluctuations and correlations in the Mott phase goes beyond the mean-field description of Mott insulators (Ch. 2).

We would like to stress that Eq. 5.1 specifies the ground-state of the system. Particlehole pairs can therefore be regarded as virtual excitations of the system. This should be distinguished from actual excited states of Mott insulators, which are reached by increasing or decreasing the total atom number by one. The corresponding excited eigenstates of the system are usually referred to as particle- or hole-excitations, respectively (Ch. 2 and Sec.9.2).

For the detection of particle-hole pairs, we exploited the fact that they are bound objects. As a consequence, the appearance of a number-fluctuation on a given site leads to an enhanced probability to find a fluctuation on a close-by site. This behavior 
is captured in a two-site parity correlation function [91]

$$
C(d)=\left\langle\hat{s}_{k} \hat{s}_{k+d}\right\rangle-\left\langle\hat{s}_{k}\right\rangle\left\langle\hat{s}_{k+d}\right\rangle,
$$

where $\hat{s}_{k}=e^{i \pi\left(\bar{n}-\hat{n}_{k}\right)}$ is the parity operator at site $k$ and $d$ is the distance between the lattice sites. For the case of an average occupation number $\bar{n}=1, \hat{s}_{k}$ yields $+1(-1)$ for an odd (even) occupation number $n_{k}$. In Ch. 4, we used a different definition of the parity operator, $\hat{P}_{k}=\frac{1}{2}\left(\hat{s}_{k}+1\right)$, which yielded $1(0)$ for an odd (even) occupation number in the case of an average occupation number $\bar{n}=1$. We used $\hat{s}_{k}$ here to be consistent with the notation for the string-order parameter introduced in Ch. 6. For two-site correlation functions, the change of the definition leads to a pure numerical factor. The two-site correlation function formulated with $\hat{s}_{k}$ is a factor of four higher than the one formulated with $\hat{P}_{k}$.

The correlation function defined in Eq. 5.2 has the important feature that it vanishes for a many-body state $|\Psi\rangle=\prod_{i}\left|\psi_{i}\right\rangle$ that is a simple product of on-site states $\left|\psi_{i}\right\rangle$. In such a case, we find $\left\langle\hat{s}_{k} \hat{s}_{k+d}\right\rangle=\left\langle\hat{s}_{k}\right\rangle\left\langle\hat{s}_{k+d}\right\rangle$ and therefore $C(d)=0$ for all $d>0$. As a consequence, we expect no signal for the states of the atomic $(J / U=0)$ and non-interacting $(U / J=0)$ limit, since they can both be expressed as a product of onsite states (Eq. 2.2 and 2.5). Also the finite temperature ensemble in the atomic limit (Ch. 4) is described by a product of on-site density operators. One can additionally show that for such systems, $C(d)=0$ for all $d>0$. We, therefore, expected no signal due to the thermally excited density excitations visible in Fig. 4.2 and Fig. 4.3.

In contrast, the perturbation theory result in Eq. 5.1 shows a many-body state that is a superposition of product states with and without a particle-hole pair at different positions. The nearest-neighbor parity-correlation function $C(d=1)$ for this state yields a positive signal of $C(d=1)=16(J / U)^{2}+\mathcal{O}\left[(J / U)^{4}\right]$. This is directly proportional to the probability to find a particle-hole pair on neighboring sites.

\subsection{Results in one dimension}

We first analyzed two-site parity correlations in one-dimensional systems. For this, we split the two-dimensional quantum gas (Sec.3.1) into parallel one-dimensional systems along the $\mathrm{x}$-direction (Fig.5.2). We then varied the tunnel coupling $\mathrm{J} / U$ within these systems.

This preparation was performed on time-scales that are much slower than ones for the typical many-body dynamics in our system $(U / h$ and $J / \hbar)$. Therefore, we expect the system to be close to the equilibrium many-body state for the final $J / U$ values. Typically, our samples contained $150-200$ atoms in order to avoid Mott insulators of occupation numbers $\bar{n}>1$. Hence, we probed the correlations in a region that is within the $\bar{n}=1$ Mott lobe for small $J / U$ values (see shaded area in Fig. 5.1).

We obtained single-site-resolved information of the corresponding on-site parity 


\section{Detection of particle-hole pairs using two-site correlation functions}
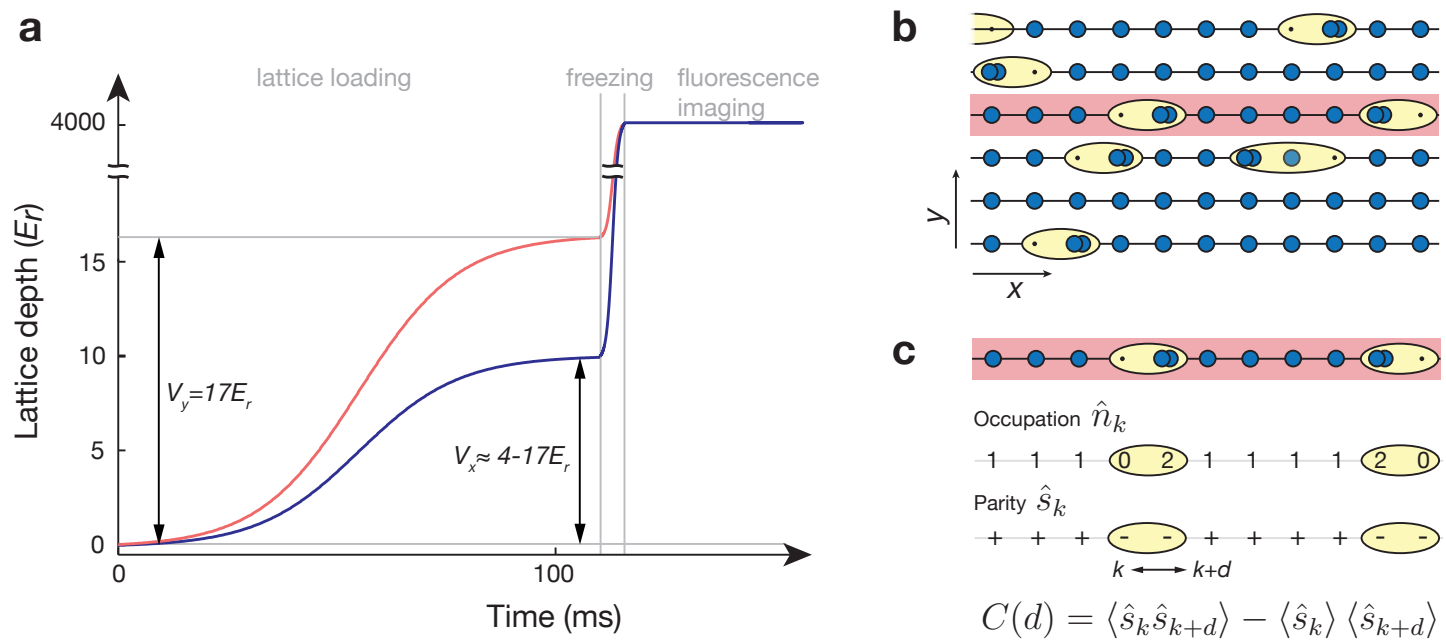

Figure 5.2.: Detection of particle-hole pairs in one-dimensional systems. a, Experimental sequence for the horizontal lattice axes. The lattice depth of the axis in the y-direction was adiabatically increased to $17 E_{r}$, which split the system into parallel one-dimensional tubes that have a negligible tunneling coupling between each other. To tune the tunneling couplings $J / U$ between the sites along the one-dimensional systems, we varied the final lattice depth of the axis in the x-direction. The ramps for both axes were s-shaped with a duration of $120 \mathrm{~ms}$. After this preparation, the density distribution was frozen and imaged with singlesite-resolved fluorescence detection (Ch.3.3 and Sec. 4.2). b, Schematic image of the density distribution for $0<J / U \ll(J / U)_{c}$. We expect parallel one-dimensional tubes with particlehole pairs aligned in the direction of the tubes. c, Analysis of a single tube highlighted in $\mathbf{b}$. The occupation number $\hat{n}_{k}$ is imaged as the parity $\hat{s}_{k}$. For the parity $\hat{s}_{k}$, we used $+1(-1)$ for odd (even) parity. To detect particle-hole pairs, we measured the two-site parity correlation functions $C(d)=\left\langle\hat{s}_{k} \hat{s}_{k+d}\right\rangle-\left\langle\hat{s}_{k}\right\rangle\left\langle\hat{s}_{k+d}\right\rangle$. In the presence of particle-hole pairs, we expect this function to show a positive signal.

distributions employing the scheme described in Sec. 3.3 and 4.2. Using this information, we evaluated $C(d)$ (Eq. 5.2) with $50-100$ experimental repetitions and by an additional average over $k$ in a central region of $9 \times 7$ lattice sites. We first evaluated $C(d=1)$ for each site in this region by an ensemble average over the experimental repetitions and then performed the spatial average. The spatially averaged signal can therefore be written as $\sum_{k \epsilon R}\left(\left\langle\hat{s}_{k} \hat{s}_{k+1}\right\rangle-\left\langle\hat{s}_{k}\right\rangle\left\langle\hat{s}_{k+1}\right\rangle\right) / N_{R}$ with $N_{R}$ the number of sites in the central region $R$.

First, we recorded the nearest-neighbor correlations $C(d=1)$ for different values of $J / U$ along the direction of the one-dimensional tubes (red circles in Fig. 5.3a). We will discuss this signal going from small $J / U$ to higher $J / U$ values. 

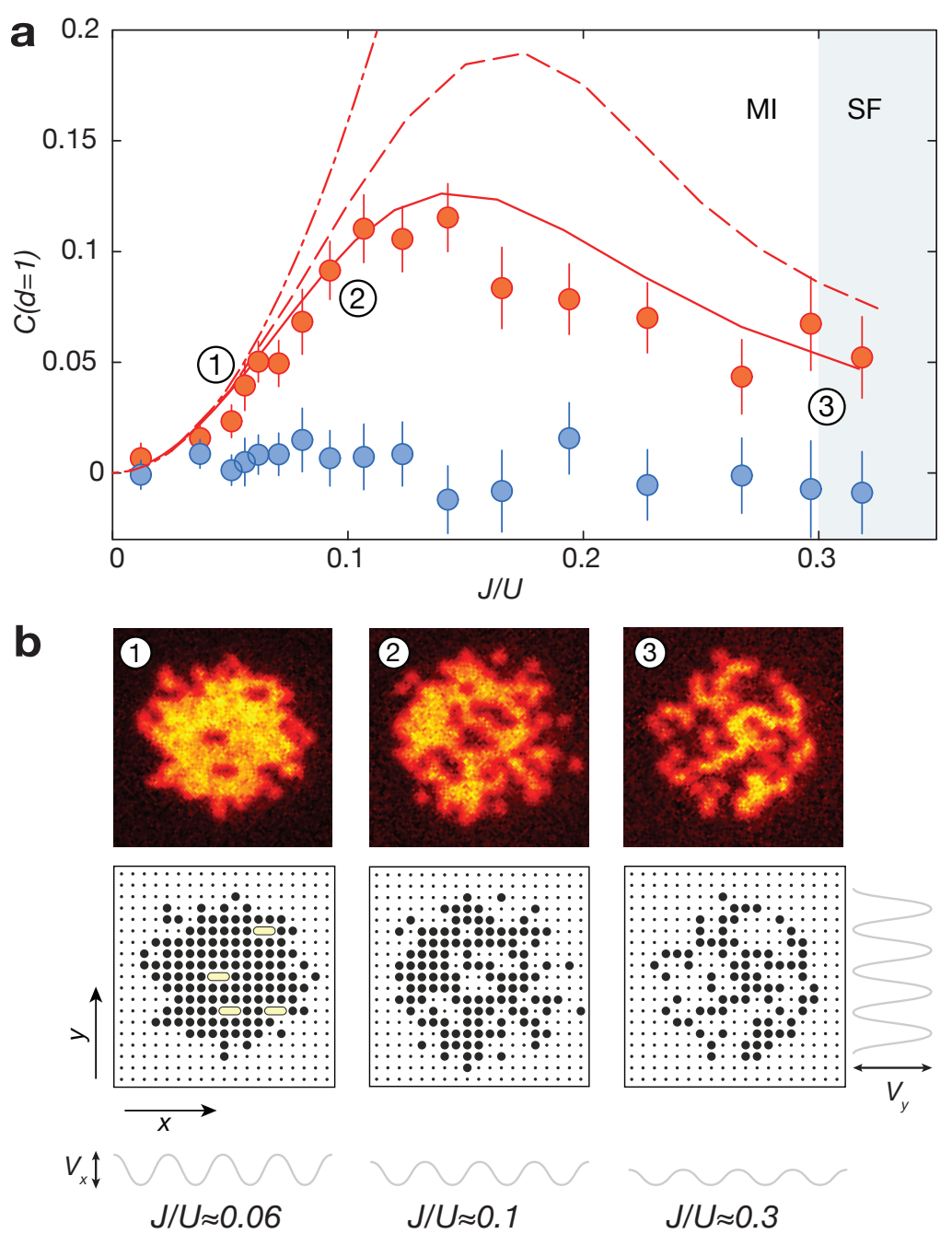

Figure 5.3.: Nearest-neighbor correlation function in one dimension. a, One-dimensional nearest-neighbor correlations $C(d=1)$ as a function of $J / U$. The data along the tube direction ( $x$-direction, red circles) shows a positive correlation signal, while the signal in orthogonal direction ( $y$-direction, blue circles) vanishes within error-bars due to the decoupling of the one-dimensional systems. For small $J / U \approx 0$, we find no correlations at all, consistent with the description of atomic limit Mott insulators as product states. For small, non-zero $(J / U) \lesssim 0.05$, the signal is consistent with first-order perturbation theory (dashed-dotted line). We find a maximum in the signal, for $J / U \approx 0.15$, which is smaller than the critical value of $(J / U)_{c}^{1 d} \approx 0.3$. The overall signal is lower than the generic signal expected in a homogeneous system at zero temperature (dashed line), calculated using Density-Matrix Renormalization Group (DMRG). Including both the harmonic confinement and a finite temperature of $T=0.09 U / k_{B}$ (solid line) yields good quantitative agreement. The latter calculation was performed using finite temperature Matrix-Product-State (MPS) simulation. The error bars denote the $1 \sigma$ statistical uncertainty. The light blue shading highlights the superfluid phase. $\mathbf{b}$, Top row: Typical experimental fluorescence images for $J / U=0.06(\mathrm{~b} 1), J / U=0.11$ (b2) and $J / U=0.3$ (b3). Bottom row: Reconstructed on-site parity. Particle-hole pairs are emphasized by a yellow shading in (b1). For increased $J / U$, the pairs start to proliferate and identification in a single experimental image becomes impossible $(b 2, b 3)$. 


\section{Detection of particle-hole pairs using two-site correlation functions}

For $J / U \approx 0$ (atomic limit), the nearest-neighbor correlations vanish within errorbars, which is compatible with the product state description of the many-body state in this limit (see discussion below Eq. 5.2). As particle-hole pairs emerge with increasing $J / U$, we observe an increase of nearest-neighbor correlations. The initial rise for small $J / U \lesssim 0.05$ values is compatible with the first-order perturbation theory from Eq. 5.2 (dashed-dotted line in Fig. 5.3a).

For larger $J / U$ values, the nearest-neighbor correlations increase further until a peak value is reached. The appearance of a maximum can be understood from the fact that $C(d)$ has to vanish also in the non-interacting limit $(U / J=0)$. Consequently, we must find a maximum of $C(d)$ for a finite $J / U$ value. The precise location and value of this maximum is, however, hard to predict. Interestingly, the maximum is reached for a $J / U$ value that is higher than the critical value in mean-field theory $(J / U)_{c, M F} \approx 0.09$ but lower than the more precise critical value $(J / U)_{c}^{1 d} \approx 0.3$ obtained with numerical methods (Ch. 2). We will see in Sec. 6.4 that a three-site correlation function shows a maximum which is closer to the critical point.

Our data show very good agreement with ab initio finite-temperature MPS calculations $[92,93]$ at temperature $T=0.09 U / k_{B}$ (Fig. 5.3a, solid line) that also take into account our harmonic trapping potential with frequency $\omega /(2 \pi) \approx 60 \mathrm{~Hz}$. We would like to stress that there is no fitting procedure involved and the simulation is completely ab initio.

Compared to a homogeneous system at $T=0$ (dashed-line), the experimental signal is reduced, especially around the maximum. This reduction can be attributed in equal parts to the finite temperature of our system and the averaging over different local chemical potentials.

Let us explain the latter point in more detail. Because of the finite region of interest and the harmonic trapping potential, we are averaging the signal from different chemical potentials $\mu$ at a fixed $J / U$. Close to the transition point, the averaged signal contains a contribution from the Mott insulator and the superfluid phase (see Fig. 5.1). Since the signal in the superfluid phase is lower, the averaged signal is reduced compared to the genuine signal in a homogeneous system at fixed density. This effect is especially severe in the one-dimensional case owing to the narrow width of the Mott lobe for $\bar{n}=1$ close to the critical point [58].

The observed signal can be considered a genuine quantum effect. Thermally induced defects in a fixed density background would also lead to an empty site (hole) and a site with an additional particle (particle). However, such thermally induced particle-hole pairs extend over arbitrary distances because both the hole and particle defect can tunnel without energy cost at $J / U>0$. Their presence leads to an increase of on-site fluctuations, but to a reduction of the parity-correlation signal as seen from the comparison with the numerical results.

This is in contrast to the measurement of phase-coherence, e.g., using time-of-flight imaging. It has been shown that the residual phase-coherence in the Mott phase 
can increase with increasing temperature, because the thermally activated defects delocalize and form extended wave packets [94].

Finally, we found no correlations when performing the same analysis perpendicular to the one-dimensional tubes (blue circles in Fig.5.3a), showing that the coupling between the tubes was indeed negligible.

\subsection{Results in two dimensions}

As the dimensionality of the system plays an important role in its correlation properties, we also measured the two-site parity correlations across the two-dimensional superfluid-Mott-insulator transition. In this case, we expect particle-hole pairs and correlations along both lattice axes and also a reduced critical value $(J / U)_{c}^{2 d} \approx 0.06$ compared to the one-dimensional case $(J / U)_{c}^{1 d} \approx 0.3$.

The experimental sequence is the same as for the measurement in the one dimensional systems (Fig. 5.2), except that both lattice axes are simultaneously tuned to the same final depth, resulting in the same $J / U$ value along both directions. The evaluation procedure for $C(d=1)$ was as described in the previous section.

In contrast to the one-dimensional case, we now observe the same nearest-neighbor correlations within error bars along both axes (blue and red circles in Fig. 5.4). The peak value is now reached around the critical value $(J / U)_{c}^{2 d} \approx 0.06$ [56] and the signal in most of the Mott phase is well described by first-order perturbation theory (dashed-dotted line in Fig. 5.4).

Additionally, we found that the peak correlation value is significantly reduced compared to the one-dimensional case. This experimentally demonstrates the fact that quantum correlations are directly influenced by the dimensionality of the system. In particular, they are more pronounced the more the system's dimensionality is reduced.

We also compared our data with Quantum Monte Carlo (QMC) simulations [41] for a homogeneous system at $T=0.1 U / k_{B}$ (solid line in Fig. 5.4) and found good quantitative agreement. Here, the broader shape of the Mott lobe leads to a weaker averaging effect over different local chemical potentials and the system is well described by the simulation for a homogeneous system.

Additionally, we find a weaker dependence of the correlation signal on the system's temperature (compare solid and dashed line in Fig. 5.4). Since the width of the Mott lobe sets the energy scale for thermal excitation (Ch. 2), this is again compatible with the broader Mott lobe in two dimensions. 


\section{Detection of particle-hole pairs using two-site correlation functions}

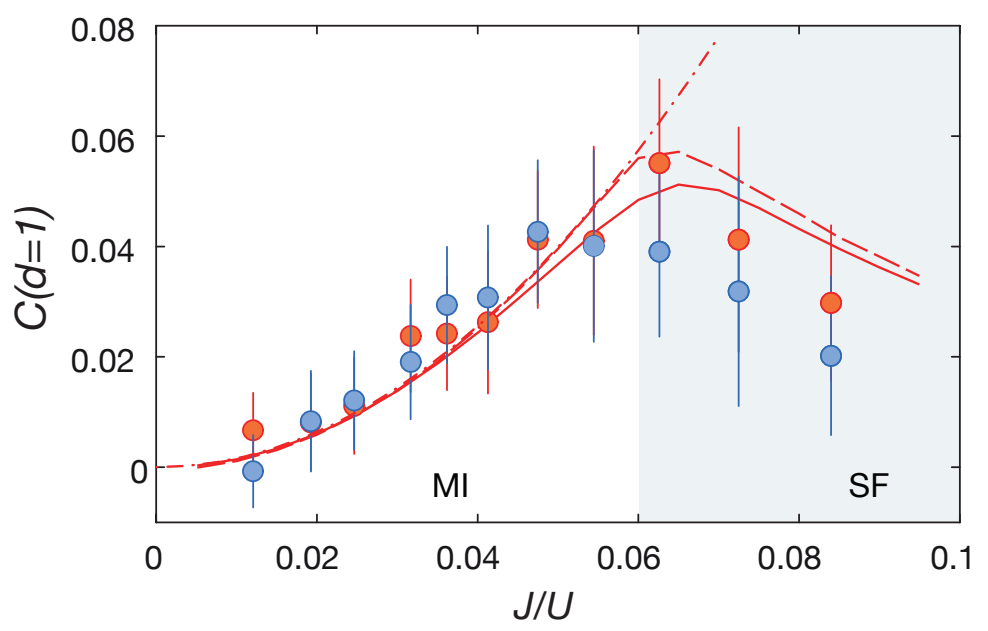

Figure 5.4.: Nearest-neighbor correlation function in two dimensions. Two-dimensional nearest-neighbor correlations $C(d=1)$ as a function of $J / U$ along the $x$ (red circles) and $y$ directions (blue circles). We now observe the same correlation signal along both axes within errorbars. The curves are first-order perturbation theory (dashed-dotted line) and a QMC calculation for a homogeneous system at $T=0.01 U / k_{B}$ (dashed line) and $T=0.1 U / k_{B}$ (solid line). The error bars denote the $1 \sigma$ statistical uncertainty. The light blue shading highlights the superfluid phase.

\subsection{On-site fluctuations and next-nearest neighbor correlations}

In addition to the nearest-neighbor parity correlation $C(d=1)$, we also evaluated the correlations $C(d)$ (Eq.5.2) for distances $d=0$ and $d=2$ in our one-dimensional systems (Fig. 5.5a). For $d=0$, this amounts to measuring the on-site variance $C(d=0)=\sigma\left(\hat{s}_{k}\right)^{2}$. Deep in the Mott-insulating regime, the on-site number distribution is strongly squeezed and the variance is close to zero, whereas in the superfluid regime, it saturates at $\sigma\left(\hat{s}_{k}\right)^{2}=1 . C(d)$ drops rapidly as a function of the distance $d$, and the numerical calculations predict only a small maximum of 0.01 in the nextnearest-neighbor correlation $C(d=2)$ at $J / U \sim 0.17$, which is however indiscernible from the statistical noise in our measurements (Fig. 5.5b).

We also show the parity correlation $C(d)$ in the two-dimensional system for different distances (Fig. 5.5c). We found that, similar to the one-dimensional systems, nextnearest-neighbor correlations are not visible above the statistical noise, whereas the QMC simulations show a small maximum around $J / U \sim 0.065$ (Fig. 5.5d). However, a striking difference is that the on-site fluctuations around the critical point are significantly smaller than in one dimension. 

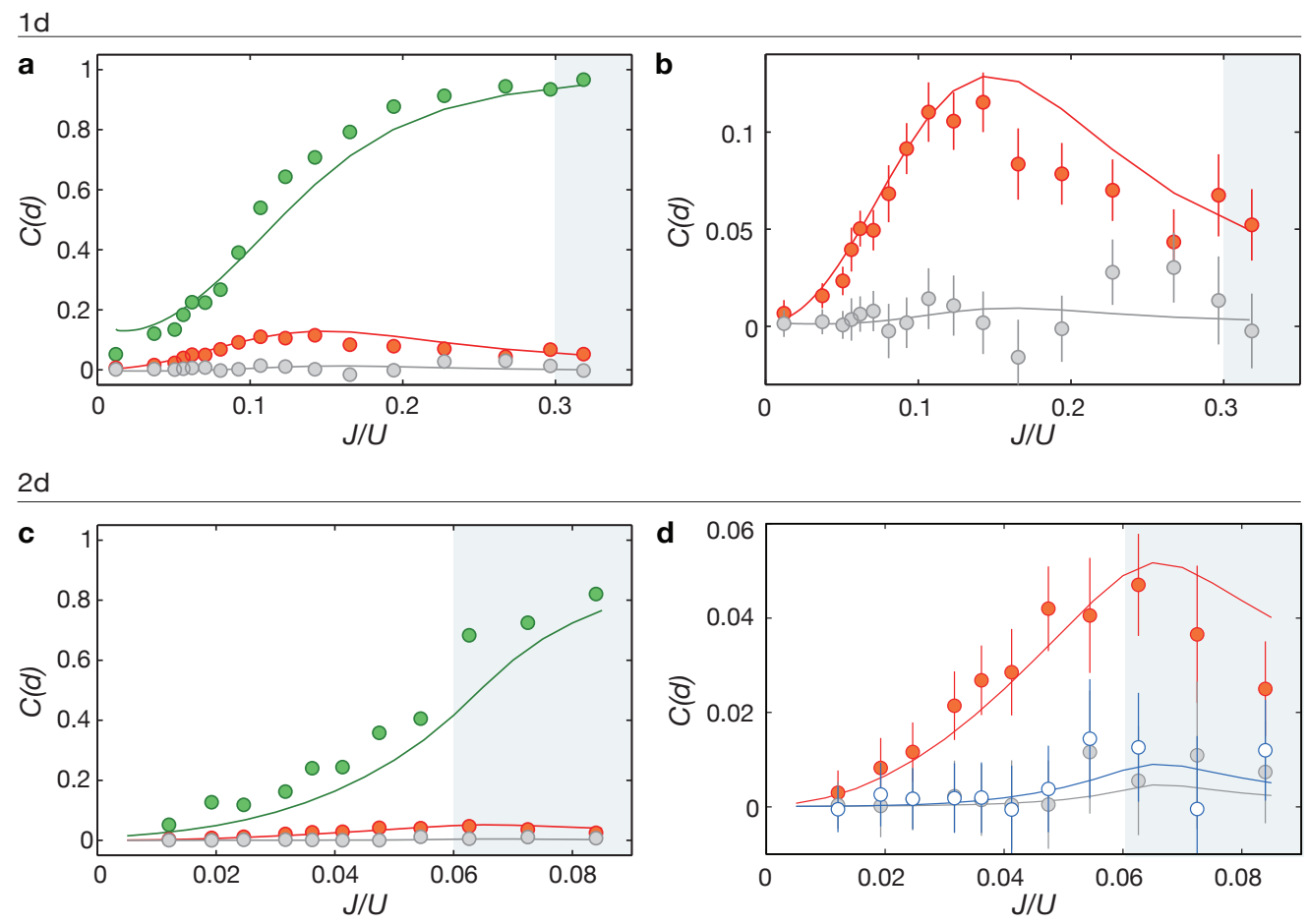

Figure 5.5.: On-site fluctuations and next-nearest neighbor correlations a, Parity correlations $C(d)$ for different distances $d=0,1,2$ (green, red and gray circles, respectively) for the one-dimensional systems. The solid lines are finite-temperature MPS calculations including harmonic confinement at $T=0.09 U / k_{B}$. $\mathbf{b}$, Enlarged view of $C(d)$ for $d=1,2$ for the same datasets as in a. c, Same quantities as in a, but for the two-dimensional case, where the data is the mean of $C(d)$ for the two directions. $\mathbf{d}$, Enlarged view of $C(d)$ for $d=1,2$ (red, gray) and additionally the two-site correlations for the next-nearest-neighbor along the diagonal (blue). The solid lines are a QMC calculation for a homogeneous system at $T=0.1 U / k_{B}$. The statistical errorbars in $\mathbf{a}$ and $\mathbf{c}$ are smaller than the dot size. We attribute the systematic shift of $C(d=0)$ in $\mathbf{c}$ to the inhomogeneous trapping potential. 


\subsection{Summary and conclusion}

We have shown single-atom- and single-site-resolved measurements of two-site parity correlation functions across the one-dimensional and two-dimensional superfluid-Mott-insulator transition. To the best of the author's knowledge, this has been the first time that a correlation function in an interacting lattice system has been directly measured with single-atom and single-site resolution. The measurement, therefore, constitutes a significant advance in the study of correlated many-body systems.

In particular, the rise of the correlation signal with increasing nearest-neighbor coupling strength directly shows the presence of correlated particle-hole pairs in the Mott-insulating phase. Additionally, our results agree quantitatively with fully $\mathrm{ab}$ initio numerical calculations for the whole range of nearest-neighbor coupling strengths. Furthermore, the correlation signal in one and two dimensions clearly differ, with the one-dimensional system showing significantly stronger on-site fluctuations and nearest-neighbor correlations within the Mott phase.

However, we found that the correlation signal quickly drops with distance. It is, therefore, a natural question to ask whether the specific type of correlation function studied in this chapter is a good choice to investigate the long distance behavior in the system. Indeed, it is generally known that two-site correlations vanish exponentially fast with distance in a gapped phase, such as the Mott insulator [95]. We will see in the following chapter that non-local correlation functions, which include information of an extended region instead of only two points, can show long-range order even in a gapped phase. 


\section{Non-local correlations in one dimension}

Non-local correlation functions characterize certain quantum phases in onedimensional systems that do not possess long-range order based on a two-point correlation function. Here we show that non-local correlation functions can be directly detected using single-atom-resolved imaging (Ch.3 and Sec.4.2). We will first give an introduction to the concept of non-local correlations (Ch. 6.1) and then investigate non-local correlations in bosonic Mott insulators in more detail. Specifically, we will present several theoretical arguments that bosonic Mott insulators in one dimension show a non-local string order (Ch. 6.2) and present our experimental results (Ch. 6.3). This is followed by a thorough analysis of the connection between string order and fully-connected multi-site correlation functions (Ch. 6.4).

\subsection{Non-local order parameters}

The following introduction to non-local order parameters follows in parts the discussion in Ref. [95].

\section{Local order parameters and two-site correlation functions}

Before dealing with non-local order parameters, let us define what we mean by a local order parameter. Local order parameters are based on two-site correlation functions

$$
C(x, y)=\left\langle\hat{A}_{x} \hat{B}_{y}\right\rangle
$$

where $\hat{A}_{x}$ and $\hat{B}_{y}$ are unspecified local operators acting at position $x$ and $y$. We say a system possesses long-range order in a two-point correlation function if

$$
\lim _{|x-y| \rightarrow \infty} C(x, y)=|C|^{2} \neq 0 .
$$

If this is the case, $C$ is called a local order parameter. This concept is widely used in physics. Typically, one studies the transition from an ordered phase with long-range order to a disordered phase with $C=0$. Examples include off-diagonal long-range order in a Bose-Einstein condensate defined as

$$
\lim _{|x-y| \rightarrow \infty}\left\langle\hat{\Psi}^{\dagger}(x) \hat{\Psi}(y)\right\rangle=|\Psi|^{2} \neq 0
$$




\section{Non-local correlations in one dimension}

where $\Psi$ is the macroscopic wave function and $\hat{\Psi}(x)\left(\hat{\Psi}^{\dagger}(x)\right)$ are annihilation (creation) operators (compare also Eq. 2.6).

The simplest example of magnetic order is Ising ferromagnetic long-range order, defined as

$$
\lim _{|i-j| \rightarrow \infty}\left\langle\hat{S}_{i}^{z} \hat{S}_{j}^{z}\right\rangle \neq 0,
$$

where $\hat{S}_{i}^{z}$ is the z-component of the spin operator (Sec. 7.2.1).

In a quasi-ordered phase in low-dimensional systems, we often find only an algebraic decay of $C(x, y)$ to zero and no finite value of $C$ (quasi-long-range order). A disordered phase is then characterized by a faster exponential decay of $C(x, y)$.

\section{Non-local order parameters}

Many phases that do not show order in the above sense still show non-vanishing long-range correlations in multi-site correlation functions of the type

$$
O(x, y)=\left\langle\hat{A}_{x}\left(\prod_{x<z<y} \hat{u}_{z}\right) \hat{B}_{y}\right\rangle .
$$

This can be thought of as an extension of the two-point correlation function with a string of operators $\prod_{x<z<y} \hat{u}_{z}$ inserted between the endpoints. We call $O(x, y)$ a nonlocal correlation function because it gathers information about an extended region of the system. A system possesses non-local order if

$$
\lim _{|x-y| \rightarrow \infty} O(x, y)=O^{2} \neq 0 .
$$

This definition is a generalization of so-called string order

$$
\lim _{\left|i^{\prime}-j\right| \rightarrow \infty}\left\langle\hat{S}_{i}^{z}\left(\prod_{i<k<j} e^{i \pi \hat{S}_{k}^{z}}\right) \hat{S}_{j}^{z}\right\rangle \neq 0,
$$

which was introduced in the context of Spin-1 chains [96, 97]. It detects an antiferromagnetic order that is hidden for a two-point correlation function (Fig. 6.1a). Following Refs. [95, 98], we will use the term string order also for the more general definition in Eq. 6.5 and Eq. 6.6 .

One of the simplest examples for non-local order is the disordered, paramagnetic phase in the one-dimensional transverse field Ising model that is characterized by

$$
\lim _{|i-j| \rightarrow \infty}\left\langle\prod_{i \leq k \leq j} \hat{S}_{k}^{x}\right\rangle \neq 0,
$$


a Spin 1 chain: hidden anti-ferromagnetic order

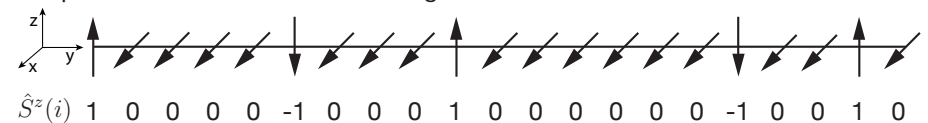

b Mott insulator: parity order

(1) no particle-hole pair cut
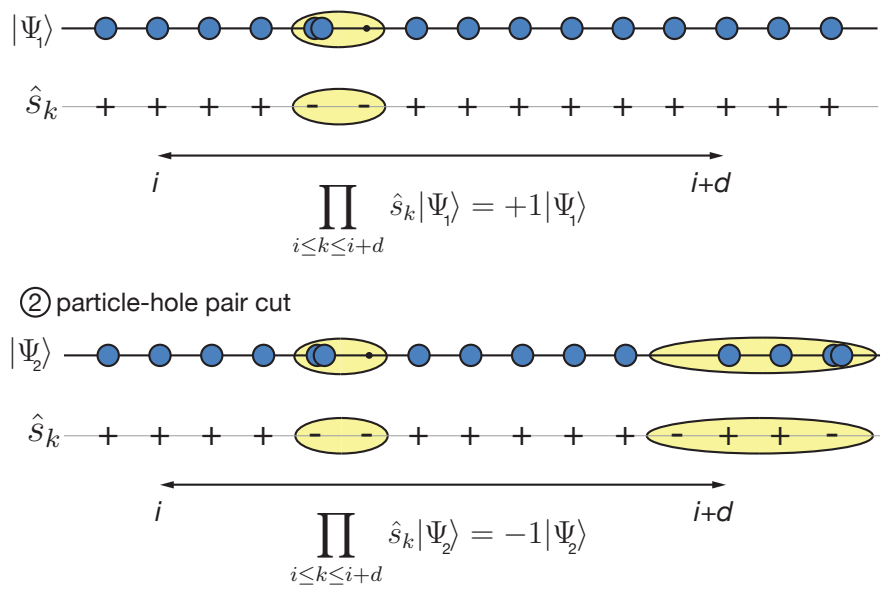

Figure 6.1.: Illustration of string order parameters. a, Hidden anti-ferromagnetic order in a Spin- 1 chain. Each spin with z-component +1 is followed by a spin with -1 , with an arbitrary number of spins with 0 in between. This order is hidden for a two-point correlation function but can be detected using a non-local correlation function (Eq. 6.7). b, Illustration of parity order in one-dimensional Mott insulators. For states where all particle-hole pairs lie within the evaluation region $[i, i+d]$, the string operator $\prod_{i \leq k \leq i+d} \hat{s}_{k}$ has an eigenvalue of +1 because the introduced pair-wise minus signs cancel (upper panel). Only states which have a particlehole pair extending out of the evaluation region yield -1 (lower panel).

where $\hat{S}_{k}^{x}$ is the x-component of the spin operator at site $k$. This is in contrast to the ordered, ferromagnetic phase of the same model, which shows long-range order in a two-point correlation function based on $\hat{S}_{k}^{z}$ (Eq. 6.4). We will explore this in more detail in Sec. 7.2.1 using the concept of dual transformations.

Our main topic is non-local order in the Bose-Hubbard model [99, 100]. We will argue in the next section that Mott insulators in one dimension exhibit a non-local order based on a correlation function

$$
\lim _{|i-j| \rightarrow \infty}\left\langle\prod_{i \leq k \leq j} e^{i \pi \delta \hat{n}_{k}}\right\rangle=\left\langle\prod_{i \leq k \leq j} \hat{s}_{k}\right\rangle \neq 0,
$$

where $\hat{s}_{k}=e^{i \pi \delta \hat{n}_{k}}$ is the on-site parity operator with $\delta n_{k}=\hat{n}_{k}-\bar{n}$ and $\bar{n}$ the average integer density. For the case of $\bar{n}=1, \hat{s}_{k}$ yields $+1(-1)$ for an odd(even) occupation number $n_{k}$. The correlation function above detects the order in the on-site parity introduced by the presence of particle-hole pairs (Sec. 6.2.1). 


\section{Non-local correlations in one dimension}

Further examples for systems with non-local order include spin-1/2 ladders [101] anisotropic two- and four-leg spin ladders [102], several phases in the fermionic Hubbard model [103, 104], and Haldane insulators in one-dimensional Bose gases [99, 100].

Recent theoretical studies explored the connection between string-order and local symmetries [98] and the connection to localizable entanglement [92, 105-107]. An alternative type of measurement different to the one presented here has been proposed in Ref. [108].

\subsection{Non-local order in the Bose-Hubbard model}

This section is devoted to a theoretical analysis of string order in the Bose-Hubbard model. We already claimed that the Mott-insulating phase in one dimensions possesses string order as defined in Eq. 6.9. For the following discussion, let us define a string operator

$$
\hat{O}(x, y)=\prod_{x \leq k \leq y} e^{i \pi \delta \hat{n}_{k}}=\prod_{x \leq k \leq y} \hat{s}_{k} .
$$

We will also use the following notation for the string correlator

$$
\mathcal{O}_{P}^{2}(l)=\langle\hat{O}(x, x+l)\rangle
$$

since we assume translation invariance. With this, we have string order if

$$
\mathcal{O}_{P}^{2}=\lim _{l \rightarrow \infty} \mathcal{O}_{P}^{2}(l) \neq 0
$$

We will support our claim in three steps. First, we present an intuitive picture for the Mott phase based on a strong-coupling expansion, which clarifies the connection between string order and particle-hole pairs. Following this, we present an explicit analytical treatment based on Bosonization and finally, present numerical data.

\subsubsection{Intuitive picture and strong coupling limit}

In the atomic limit $(J / U=0)$ and at zero temperature, we found that the Mottinsulating ground state has the form $|\Psi\rangle_{J / U=0}=\prod_{i}\left|n_{i}=1\right\rangle$. In this limit, one trivially finds $\langle\hat{O}(x, y)\rangle=1$ and therefore string order. We will now demonstrate that string order is stable with respect to finite tunneling $J / U>0$. For clarity of presentation, let us repeat the first-order strong coupling expansion

$$
|\Psi\rangle_{J / U \ll(J / U)_{c}} \propto|\Psi\rangle_{J / U=0}+\frac{J}{U} \sum_{\langle i, j\rangle} \hat{b}_{i}^{\dagger} \hat{b}_{j}|\Psi\rangle_{J / U=0}
$$




\subsection{Non-local order in the Bose-Hubbard model}

already presented in Eq. 5.1. It describes the Mott state locally for small $J / U$.

All states that are summed up in the expansion are eigenstates of the parity operator $\hat{s}_{k}$. If no defect is encountered at site $k, \hat{s}_{k}$ has an eigenvalue $s_{k}=+1$ and if a defect is encountered we find $s_{k}=-1$. Therefore, the states in the expansion on the right hand site of Eq. 6.13 are eigenstates of $\hat{O}(x, y)$ with eigenvalues $\prod_{x \leq k \leq y} s_{k}= \pm 1$. The sign of the eigenvalue depends on whether or not a particle-hole pair is cut at the ends of the string operator $\hat{O}(x, y)$. With this we mean the following. Consider a state $\hat{b}_{i}^{+} \hat{b}_{j}|\Psi\rangle_{J / U=0}$, which has a particle-hole pair at positions $i$, $j$, where $i$ is the position of the particle and $j$ the position of the hole. This pair is cut if either the particle $i$ or the hole position $j$ is within the interval $[x, y]$ but not both. If a single pair is cut, the eigenvalue of $\hat{O}(x, y)$ is -1 , because we find an unpaired minus sign. In all other cases, the eigenvalue is +1 because particle-hole pairs inside $[x, y]$ lead to pairwise minus signs that cancel (Fig. 6.1).

In the expansion of Eq. 6.13, we find four different states for which such a cut is possible, because there are two endpoints of the string operator and two permutations of particle and hole. Each of these states appears with a quantum mechanical amplitude of $\sqrt{2} J / U$. (The factor $\sqrt{2}$ stems from $\hat{b}_{i}^{\dagger}|1>=\sqrt{2}| 2>$.) The total probability to cut a particle-hole pair is therefore $p_{c}=4(\sqrt{2} J / U)^{2}=8(J / U)^{2}$ and the total probability to not cut a particle-hole pair is $p_{n c}=1-8(J / U)^{2}$. Based on the argument of the previous paragraph, we can write

$$
\mathcal{O}_{P}^{2}(l)=\langle\hat{O}(x, y)\rangle=p_{n c}-p_{c}=1-16(J / U)^{2} .
$$

This result is valid if $|x-y| \geq 2$ and for small $J / U \ll(J / U)_{c}$. Consequently, we find $\lim _{l \rightarrow \infty}\left\langle\mathcal{O}_{P}^{2}(l)\right\rangle=1-16(J / U)^{2}>0$. Therefore, one-dimensional bosonic Mott insulators at small $\mathrm{J} / U$ possess string order.

The previous analysis is expected to hold in a similar way in the whole Mott phase if higher order terms in the perturbation series are included. The crucial point is that for a given order of $(J / U)^{n}$, in the Mott-insulating phase, defects appear in finite-size clusters with a typical extension $l_{c}$. For low orders of $n$, we expect $l_{c} \approx n$. Within such clusters, the number of lattice sites with eigenvalue $s_{k}=-1$ is always even because the application of the perturbation operator $\hat{b}_{i}^{\dagger} b_{j}$, with $i, j$ being neighboring sites, can only change this number by \pm 2 . For example, the application of $\hat{b}_{i}^{\dagger} b_{j}$ on a state $|1,1, \ldots, 1,0,2,1 . ., 1,1\rangle$ can create another particle-hole pair, destroy the particle-hole pair, or lead to states $|1,1, \ldots, 0,1,2, \ldots, 1,1\rangle$ or $|1,1, \ldots, 0,3,0, \ldots, 1,1\rangle$. In all cases, we find an even number of sites with $s_{k}=-1$. If such a finite-size cluster lies completely within the evaluation region $[x, y]$ it contributes only with an overall plus sign, since the contribution of all sites with $s_{k}=-1$ cancels pairwise. The string correlation function $\mathcal{O}_{P}^{2}(l)$ then takes on a constant value if $l \gg l_{c}$, because in this case, clusters can only be cut at the ends of the evaluation region and the probability 


\section{Non-local correlations in one dimension}

for such a cut stays constant as a function of the length $l$.

In contrast, the state for the non-interacting limit can be written as a product of on-site coherent states (Eq. 2.5). Therefore, we have $\langle\hat{O}(x, y)\rangle=\prod_{x \leq k \leq y}\left\langle\hat{s}_{k}\right\rangle$. Since $\left\langle\hat{s}_{k}\right\rangle<1$, we find $\lim _{|x-y| \rightarrow \infty}\langle\hat{O}(x, y)\rangle=0$. We will see in the following section that this result is not completely correct in an interacting one-dimensional superfluid. We will still find $\lim _{|x-y| \rightarrow \infty}\langle\hat{O}(x, y)\rangle=0$, although with an algebraic instead of an exponential decay with $|x-y|$.

\subsubsection{Analytical results based on Bosonization}

\section{Superfluid phase}

A convenient description for the low-energy physics of the superfluid phase in one dimension is given by the Luttinger-liquid Hamiltonian

$$
\hat{H}_{L L}=\frac{1}{2 \pi} \int_{-\infty}^{+\infty} d x\left[v K(\pi \hat{\Pi}(x))^{2}+\frac{v}{K}\left(\frac{\partial \hat{\phi}(x)}{\partial x}\right)^{2}\right],
$$

where $K$ is the Luttinger-liquid parameter and $v$ the sound velocity. Both coefficients $K$ and $v$ must, in general, be determined using numerical simulations.

One can derive $\hat{H}_{L L}$ from the original Hamiltonian using a Bosonization-technique $[18,109-113]$. Loosely speaking, $\hat{\Pi}$ and $\hat{\phi}$ are related to phase and density fluctuations, respectively. More precisely, the creation (annihilation) operator can be written as $\hat{\Psi}^{\dagger}(x)=\hat{n}(x)^{1 / 2} e^{-i \hat{\Theta}(x)}\left(\hat{\Psi}=\hat{n}(x)^{1 / 2} e^{i \hat{\Theta}(x)}\right)$ in terms of a density $\hat{n}(x)$ and phase operator $\hat{\Theta}(x)$. We then have

$$
\delta \hat{n}(x)=\hat{n}(x)-\bar{n} \approx \frac{1}{\pi} \frac{\partial \hat{\phi}}{\partial x}
$$

considering only long-wavelength fluctuations and $\hat{\Pi}(x)=\frac{1}{\pi} \frac{\partial \hat{\Theta}(x)}{\partial x}$.

The Hamiltonian $\hat{H}_{L L}$ can be diagonalized yielding a linear, gapless dispersion relation (App.C).

The string order parameter (Eq. 6.9) has a particularly simple form in Bosonization language. Naively, one would get

$$
\left\langle e^{i \pi \sum_{x \leq k \leq x+l} \delta \hat{n}_{k}}\right\rangle \approx\left\langle e^{i \pi \int_{x}^{x+l} d z \frac{1}{\pi} \frac{\partial \hat{\phi}}{\partial z}}\right\rangle=\left\langle e^{i(\hat{\phi}(x)-\hat{\phi}(x+l))}\right\rangle=\left\langle e^{i \hat{\phi}(x)} e^{-i \hat{\phi}(x+l))}\right\rangle .
$$

However, the authors of Ref. [100] argue that the resulting operator has to be symmetrized, which yields

$$
\mathcal{O}_{P}^{2}(l)=\langle\cos (\hat{\phi}(x)) \cos (\hat{\phi}(x+l))\rangle .
$$




\subsection{Non-local order in the Bose-Hubbard model}

Both formulas show that non-local string correlations turn into local correlations in the Bosonization language, since only $\hat{\phi}(x)$ and $\hat{\phi}(x+l)$ appear.

Based on these formulas, a calculation of $\mathcal{O}_{P}^{2}(l)$ in the ground state of the Luttingerliquid Hamiltonian is possible (App.C), which yields

$$
\mathcal{O}_{P}^{2}(l) \propto l^{-\frac{K}{2}}
$$

for large $l$. This shows an algebraic decay of $\mathcal{O}_{P}^{2}(l)$ with string-length $l$. Thus, the superfluid state shows no string order in this description.

\section{Mott-insulating state}

The Mott-insulating state can also be described using the Bosonization technique [18, $100,111,112,114]$. The resulting Hamiltonian is the Sine-Gordon Hamiltonian

$$
\hat{H}_{S G}=\hat{H}_{L L}-\frac{g}{2 \pi} \int_{-\infty}^{+\infty} d x \cos (2 \hat{\phi}(x))
$$

where $\hat{H}_{L L}$ is the Luttinger-liquid Hamiltonian from Eq. 6.15. The additional cosine term describes the influence of the lattice potential and leads to a gapped spectrum (App.C). It can be shown that this term is irrelevant for the long-distance behaviour of correlation functions in the superfluid phase (see, e.g., Ref. [111]). For small $g$, we find a Mott phase for $K<2$ and a superfluid phase $K>2$.

The string correlator can be evaluated within a harmonic approximation of the cosine term (App.C) yielding

$$
\mathcal{O}_{P}^{2}(l) \approx A \cosh \left(\frac{K}{2} K_{o}(\tilde{m} l)\right)
$$

where $K_{0}(x)$ is the zeroth modified Bessel function of second kind, $\tilde{m}=(2 g K / v)^{1 / 2}$ is the energy gap in normalized units, such that $1 / \tilde{m}=\xi$ is the correlation length, and $A$ is a constant that depends on the gap value $\tilde{m}$.

Importantly, we find that $A>0$ as long as $\tilde{m} \neq 0$ and that $\lim _{l \rightarrow \infty} \cosh \left(\frac{K}{2} K_{o}(\tilde{m} l)\right)=$ 1 (App.C). Therefore, we have in the Mott-insulating phase $\lim _{l \rightarrow \infty} \mathcal{O}_{P}^{2}(l)>0$, which means that one-dimensional bosonic Mott insulators show string order in the Bosonization description, within harmonic approximation of the cosine term of the Hamiltonian.

The term $\cosh \left(\frac{K}{2} K_{o}(\tilde{m} l)\right)$ yields the functional dependence of $\mathcal{O}_{P}^{2}(l)$ on the length $l$. One can show that for short lengths $l \ll \xi$

$$
\mathcal{O}_{P}^{2}(l) \propto l^{-K / 2}
$$




\section{Non-local correlations in one dimension}

We thus recover the behavior of the superfluid phase on length scales that are short compared to the correlation length $\xi$.

For $l \gg \xi$, we have

$$
\mathcal{O}_{P}^{2}(l) \propto\left(1+\frac{\pi K^{2}}{16} e^{-2 \tilde{m} l} \frac{1}{\tilde{m} l}\right),
$$

which shows an essentially exponential decay.

The full form of $\cosh \left(\frac{K}{2} K_{o}(\tilde{m} l)\right)$ interpolates between the algebraic decay for short lengths and the exponential decay to a finite value for long lengths. The transition between both forms occurs on a length-scale proportional to $1 / \tilde{m}=\xi$. At the phase transition point, when the gap $\tilde{m}$ closes, $\tilde{\xi}$ diverges and the formula shows a purely algebraic decay in the superfluid phase. In the following section, we will compare this result with numerical simulations.

\subsubsection{Numerical analysis}

We present a numerical analysis using DMRG in Fig. 6.2, which shows the result for $\mathcal{O}_{P}^{2}(l)$ for selected lengths $l$ (solid lines). We compare this data with the result from the strong coupling expansion $\mathcal{O}_{P}^{2} \approx 1-16(J / U)^{2}$ (dashed line) and find good agreement for small $J / U$ values. The inset shows the extrapolated values $\mathcal{O}_{P}^{2}=\lim _{l \rightarrow \infty} \mathcal{O}_{P}^{2}(l)$, which we calculated using finite size scaling (App. B). We observe that $\mathcal{O}_{P}^{2}>0$ in the Mott phase and that $\mathcal{O}_{P}^{2}$ vanishes at $J / U \approx 0.3$, which is compatible with the known numerical value of $(J / U)_{c} \approx 0.3$ for the critical coupling strength for the one-dimensional superfluid-Mott-insulator transition (Ch. 2). This analysis shows that one-dimensional bosonic Mott insulators possess string order for all coupling strengths $J / U<(J / U)_{c}$.

Without going into details, we note that a mapping to a two-dimensional interface model showed that $\mathcal{O}_{P}^{2}$ grows as $\mathcal{O}_{P}^{2} \propto \exp \left(-A\left[(J / U)_{c}^{1 d}-(J / U)\right]^{-1 / 2}\right)$ at the transition point [115] and our numerical data is compatible with this behavior (Inset of Fig. 6.2). The one-dimensional superfluid-Mott-insulator transition is of the Berezinskii-Kosterlitz-Thouless type, and the latter scaling is the same scaling as for the opening of the Mott-insulating gap at the transition point [116].

We analyze the behavior of $\mathcal{O}_{P}^{2}(l)$ as a function of $l$ in Fig. 6.3a, where we show $\mathcal{O}_{P}^{2}(l)$ for three different couplings $J / U$ in a log-log plot. An algebraic decay, which appears linear in the log-log plot, is visible for short lengths $l$. As we approach the transition at $(J / U)_{c} \approx 0.3$, we observe that $\mathcal{O}_{P}^{2}(l)$ stays algebraic for longer lengths $l$ as a result of the increasing correlation length.

A fit of $\mathcal{O}_{P}^{2}(l)$ with the Bosonization result from Eq.6.21 (solid line in Fig. 6.3a) yields a very good result. The fitting parameters as a function of $J / U$ are shown in 


\subsection{Non-local order in the Bose-Hubbard model}

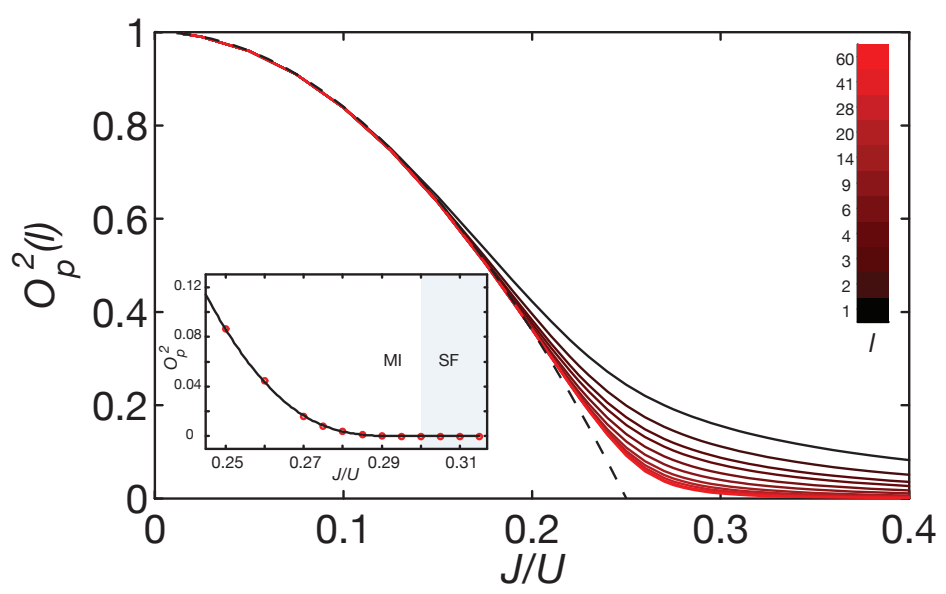

Figure 6.2.: Numerical calculation of the string-order parameter. $\mathcal{O}_{P}^{2}(l)$ as a function of $J / U$ calculated with DMRG for a homogeneous chain $(\bar{n}=1, T=0)$ of total length 216. Lines show $\mathcal{O}_{P}^{2}(l)$ for selected lengths $l$ (black to red colors). Dashed line shows first-order strong coupling result $\mathcal{O}_{P}^{2}(l)=1-16(\mathrm{~J} / U)^{2}$. Inset: Extrapolated value $\mathcal{O}_{P}^{2}=\lim _{l \rightarrow \infty} \mathcal{O}_{P}^{2}(l)$ together with a fit (black line) of the form $\mathcal{O}_{P}^{2} \propto \exp \left(-A\left[(J / U)_{c}^{1 d}-(J / U)\right]^{-1 / 2}\right)$. The fitted $(J / U)_{c} \approx 0.3$ is compatible with the known numerical value (Ch. 2$)$.

Fig. 6.3b-d. As expected, the fitted correlation length $\xi=1 / \tilde{m}(6.3 b)$ increases with $J / U$, although we find no divergence at $J / U \approx 0.3$, which we attribute to the finite system size.

The fit also yields a value for the Luttinger-liquid parameter $K$ (6.3c). We find an increasing value of $K$ with $J / U$ and $K \approx 2$ at $J / U \approx 0.3$, which is compatible with the known critical values. We interpret a small systematic shift to be the result of the finite system size. A thorough finite-size scaling for this analysis is, however, beyond the scope of this thesis. We conclude that the result from the Bosonization analysis in Eq. 6.21 gives a good description of the functional dependence of $\mathcal{O}_{P}^{2}(l)$ on $l$ even close to the critical point, which might be surprising considering the fact that the result is based on a harmonic approximation of the cosine term in Eq. 6.20. 


\section{Non-local correlations in one dimension}
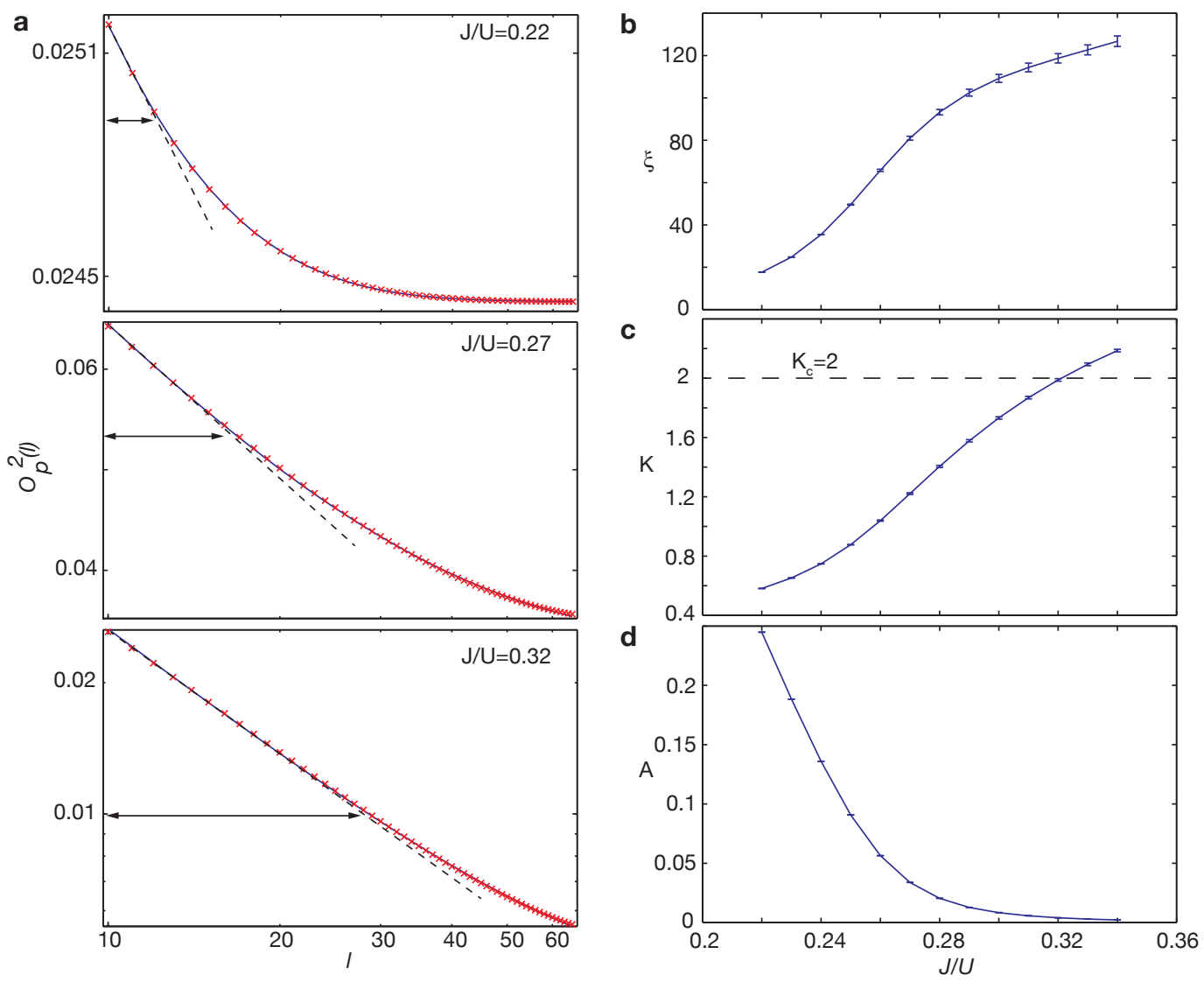

Figure 6.3.: Comparison of numerical and analytical calculation. a, $\mathcal{O}_{P}^{2}(l)$ as a function of $l$ for $J / U=0.22,0.27,0.32$ in double-logarithmic plot calculated with DMRG (crosses) for a homogeneous chain $(\bar{n}=1, T=0)$ of total length 216 . Black arrows indicate the region in which $\mathcal{O}_{P}^{2}(l)$ decays approximately algebraically. This region grows when approaching the phase transition. The straight, dashed lines serve as guides to compare the data with a strict algebraic scaling. Solid lines show a fit with the Bosonization expression from Eq. 6.21. b-d, Fitted parameters $\xi=1 / \tilde{m}, K$ and $A$ as a function of $J / U$. Errorbars denote the fit error.

\subsection{Experimental results for the string order parameter}

Our experimentally obtained values of $\mathcal{O}_{P}^{2}(l)$ for string lengths $l \leq 8$ are shown in Fig. 6.4a. They agree qualitatively well with in-trap MPS calculations at $T=$ $0.09 U / k_{B}$ (Fig. 6.4b). We observe a stronger decay of $\mathcal{O}_{P}^{2}(l)$ with $l$ compared to the $T=0$ case because at finite temperature, thermal fluctuations lead to minus signs at random positions of the chain and reduce the average value of $\mathcal{O}_{P}^{2}(l)$. Despite this, we still see a strong growth of $\mathcal{O}_{P}^{2}(l)$ once the transition from the superfluid to the Mott insulator is crossed, with a similar behavior as in Fig. 6.2.

For a completely uncorrelated state, $\mathcal{O}_{P}^{2}(l)$ factorizes to $\prod_{k \leq j \leq k+l}\left\langle\hat{s}_{j}\right\rangle$, and, in a homogeneous system, we would expect a decay with string length of the form $\left\langle\hat{s}_{j}\right\rangle^{l+1}$, 


\subsection{Experimental results for the string order parameter}
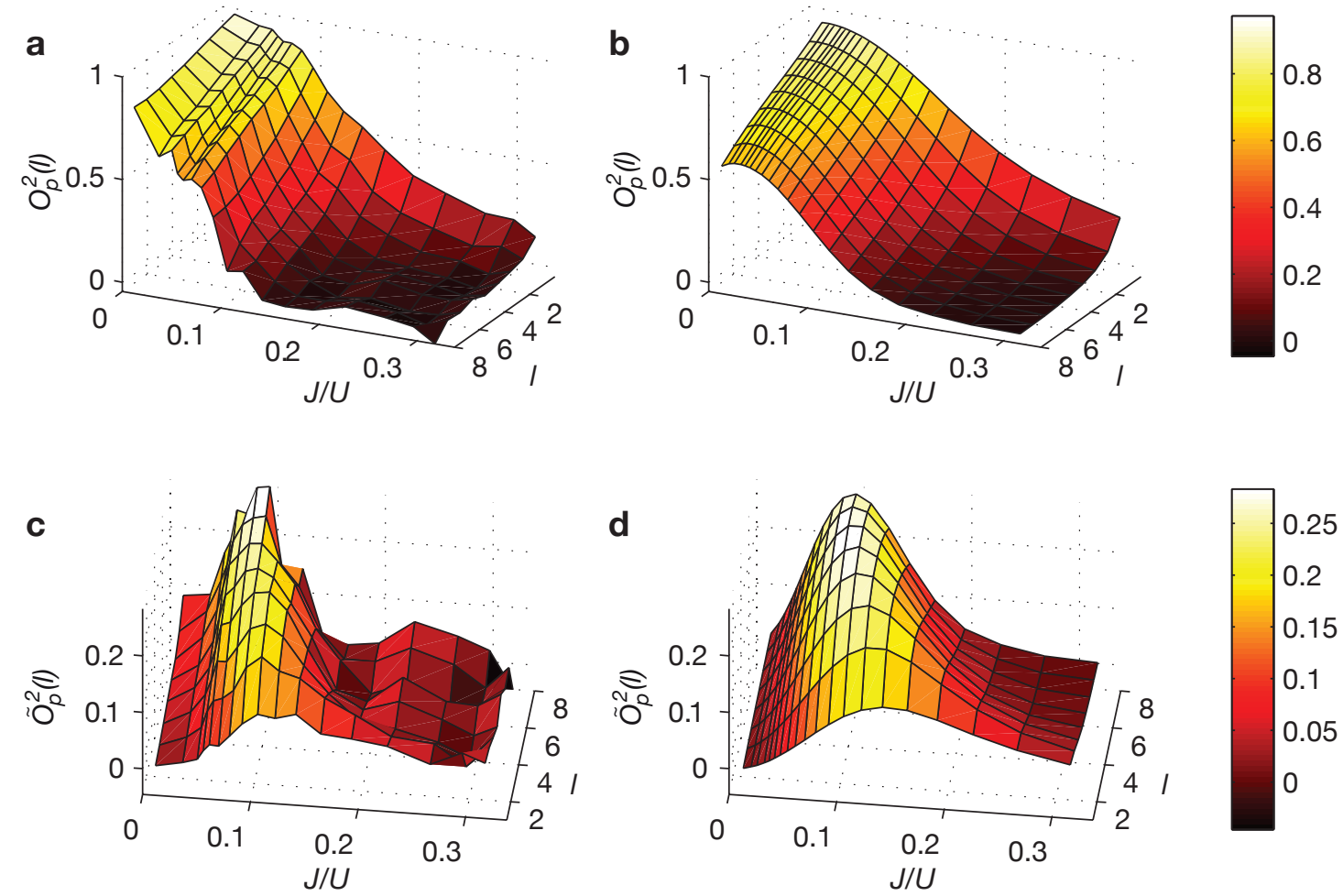

Figure 6.4.: String order results. $\mathbf{a}$, Experimental values of $\mathcal{O}_{P}^{2}(l)$ for lengths $0 \leq l \leq 8 \mathbf{b}$, Intrap MPS calculations at $T=0.09 U / k_{B}$. c, Experimentally determined string correlator $\tilde{\mathcal{O}}_{P}^{2}(l)$ as defined in Eq. 6.24 for lengths $1 \leq l \leq 8 \mathrm{~d}$, In-trap MPS calculations at $T=0.09 U / k_{B}$. The $l$ axes in $\mathbf{c}$ and $\mathbf{d}$ have been inverted.

which can be slow provided the mean on-site parity $\left\langle\hat{s}_{j}\right\rangle$ is close to one. To rule out that our experimental data shows only such a trivial behavior, we define a new quantity $\tilde{\mathcal{O}}_{P}^{2}(l)$ that more naturally reflects the underlying correlations:

$$
\tilde{\mathcal{O}}_{P}^{2}(l)=\mathcal{O}_{P}^{2}(l)-\prod_{k \leq j \leq k+l}\left\langle\hat{s}_{j}\right\rangle .
$$

First, we notice that $\tilde{\mathcal{O}}_{P}^{2}(l)$ for length $l=1$ is equal to the two-site correlation function $C(d=1)$ (Eq. 5.2). Second, $\tilde{\mathcal{O}}_{P}^{2}(l) \approx \mathcal{O}_{P}^{2}(l)$ for long distances $l$ since $\prod_{k \leq j \leq k+l}\left\langle\hat{s}_{j}\right\rangle$ eventually decays to zero (except for the singular case $J / U=0$ and $T=0$ ). The correlation function $\tilde{\mathcal{O}}_{P}^{2}(l)$ can therefore be understood as an extension of the two-site correlation function, which essentially captures the physics behind string order in one-dimensional Mott insulators.

Experimental and theoretical values for $\tilde{\mathcal{O}}_{P}^{2}(l)$ are shown in Figs. $6.4 \mathrm{c}$ and $\mathrm{d}$. For small $J / U, \widetilde{\mathcal{O}}_{P}^{2}(l)$ is significantly reduced compared to $\mathcal{O}_{P}^{2}(l)$ since few particlehole pairs exist and $\mathcal{O}_{P}^{2}(l)$ is close to its factorized form for short lengths $l$. In the case of vanishing $J / U$, we even expect $\tilde{\mathcal{O}}_{P}^{2}(l)=0$ since all sites are completely decoupled. 


\section{Non-local correlations in one dimension}

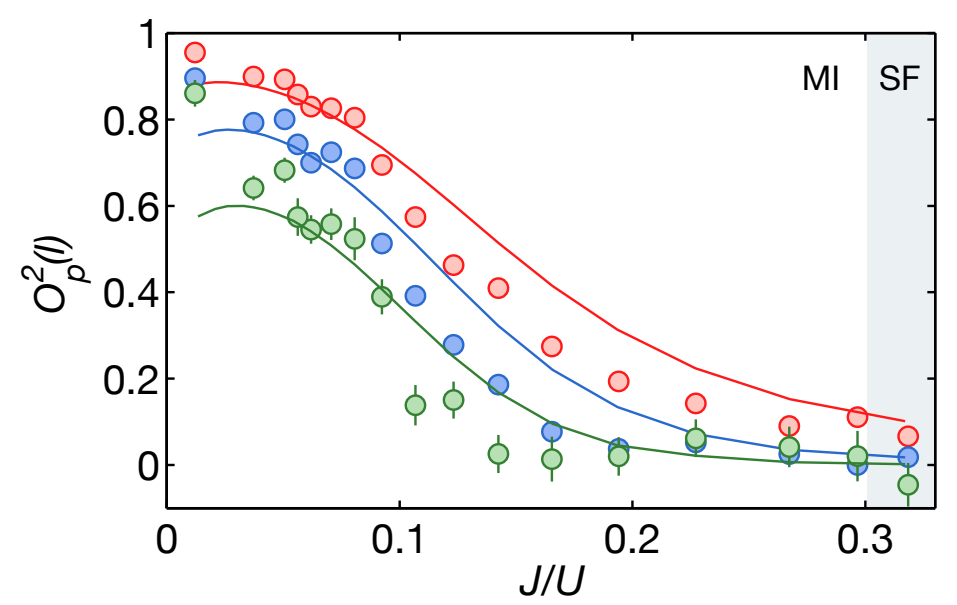

Figure 6.5.: String order quantitative comparison. String correlator $\mathcal{O}_{P}^{2}(l)$ for different lengths $l=1$ (red circles), $l=4$ (blue circles) and $l=8$ (green circles). These curves are cuts of the three-dimensional representation of the data, as shown in Fig. 6.4. Solid lines correspond to finite-temperature MPS calculations including harmonic confinement at $T=0.09 U / k_{B}$ with the same color coding as the experimental data.

For intermediate $J / U \approx 0.1, \tilde{\mathcal{O}}_{P}^{2}(l)$ grows rapidly with length $l$ showing a strong deviation from the factorized form. Finally, in the superfluid regime, $\tilde{\mathcal{O}}_{P}^{2}(l)$ becomes indiscernible from zero for large lengths, in contrast to the nearest-neighbor two-site correlation function.

For a more detailed comparison of the experimentally obtained string correlations with theory, we show $\mathcal{O}_{P}^{2}(l)$ for different lengths $l=1$ (Fig. 6.5, red circles), $l=4$ (blue circles) and $l=8$ (green circles) together with MPS calculations at $T=0.09 U / k_{B}$ including the harmonic confinement. We observe a good qualitative agreement of the data with the numerical simulations. Systematic errors of the experimental data can arise from different mean atom numbers for different $J / U$, leading to different local chemical potentials. A systematic discrepancy between theory and experiment can also result from a small mismatch of the trapping frequencies or the calibration of $J$ and $U$. Additionally, the theoretical prediction is calculated at fixed temperature (in units of $U$ ), but the experimental data is approximately taken at a constant entropy. In the latter case, we expect the temperature to scale with $U$ for small $J / U$ values, but it is not clear that this scaling remains valid for higher $J / U$ values. The temperature for the numerical simulation was chosen to yield the best agreement over the full range of $J / U$ values. 


\subsection{Multi-site correlations}

In the following paragraph, we show that our results for the string correlators cannot be explained with pure two-site correlations. Additionally, we address the connection between string order and multi-site correlations.

To illustrate this, let us consider the simplest case of a string-type correlator including three sites $\left\langle\hat{s}_{1} \hat{s}_{2} \hat{s}_{3}\right\rangle$, where $\hat{s}_{1}, \hat{s}_{2}$ and $\hat{s}_{3}$ refer to the parity of three neighboring sites on a one-dimensional chain. If one of the sites is not correlated with the others, we can calculate $\left\langle\hat{s}_{1} \hat{s}_{2} \hat{s}_{3}\right\rangle$ from two-site and on-site terms. For instance, if site 3 is not correlated with sites 1 and 2 , we have $\left\langle\hat{s}_{1} \hat{s}_{2} \hat{s}_{3}\right\rangle=\left\langle\hat{s}_{1} \hat{s}_{2}\right\rangle\left\langle\hat{s}_{3}\right\rangle$. This fact can be generally expressed using a three-site cumulant $\left\langle\hat{s}_{1} \hat{s}_{2} \hat{s}_{3}\right\rangle_{c}$, defined as [117]

$$
\left\langle\hat{s}_{1} \hat{s}_{2} \hat{s}_{3}\right\rangle_{c}=\left\langle\hat{s}_{1} \hat{s}_{2} \hat{s}_{3}\right\rangle-\left\langle\hat{s}_{1}\right\rangle\left\langle\hat{s}_{2}\right\rangle\left\langle\hat{s}_{3}\right\rangle-C_{1,2}\left\langle\hat{s}_{3}\right\rangle-C_{2,3}\left\langle\hat{s}_{1}\right\rangle-C_{1,3}\left\langle\hat{s}_{2}\right\rangle,
$$

with two-site correlation functions $C_{i, j}=\left\langle\hat{s}_{i} \hat{s}_{j}\right\rangle-\left\langle\hat{s}_{i}\right\rangle\left\langle\hat{s}_{j}\right\rangle$. The cumulant is a measure of the correlations between all three sites as it vanishes if one of the sites is not correlated with the others [117]. Additionally, if the cumulant vanishes, Eq. 6.25 can be written as $\left\langle\hat{s}_{1} \hat{s}_{2} \hat{s}_{3}\right\rangle=\left\langle\hat{s}_{1}\right\rangle\left\langle\hat{s}_{2}\right\rangle\left\langle\hat{s}_{3}\right\rangle+C_{1,2}\left\langle\hat{s}_{3}\right\rangle+C_{2,3}\left\langle\hat{s}_{1}\right\rangle+C_{1,3}\left\langle\hat{s}_{2}\right\rangle$ and we therefore have a situation where $\left\langle\hat{s}_{1} \hat{s}_{2} \hat{s}_{3}\right\rangle$ does not contain more information than two-site and on-site terms.

Our experimental values for the three-site cumulant $\left\langle\hat{s}_{1} \hat{s}_{2} \hat{s}_{3}\right\rangle_{c}$ (Fig.6.6) show a significant signal for $J / U<0.20$, in quantitative agreement with MPS calculations at $T=0.09 \mathrm{U} / \mathrm{k}_{\mathrm{B}}$ including the harmonic confinement. This is a substantial effect, as the peak value constitutes about $40 \%$ of the peak value of $\tilde{\mathcal{O}}_{P}^{2}(l=2)$, where only on-site terms have been subtracted. We draw two conclusions from this. First, our data shows a three-site correlation beyond simple two-site correlations. Second, our results for string-type expectation values cannot be expressed in terms of two-site correlations alone.

Let us explain the latter statement in more detail. It turns out that if a three-site expectation value cannot be expressed via two-site terms, then expectation values including more than three sites cannot be expressed in this way either. The four-site term, e.g., can be written as

$$
\begin{aligned}
\left\langle\hat{s}_{1} \hat{s}_{2} \hat{s}_{3} \hat{s}_{4}\right\rangle= & \left\langle\hat{s}_{1} \hat{s}_{2} \hat{s}_{3} \hat{s}_{4}\right\rangle_{c}+\left\langle\hat{s}_{1} \hat{s}_{2} \hat{s}_{3}\right\rangle_{c}\left\langle\hat{s}_{4}\right\rangle+\left\langle\hat{s}_{2} \hat{s}_{3} \hat{s}_{4}\right\rangle_{c}\left\langle\hat{s}_{1}\right\rangle \\
& +\left\langle\hat{s}_{1} \hat{s}_{2} \hat{s}_{4}\right\rangle_{c}\left\langle\hat{s}_{3}\right\rangle+\left\langle\hat{s}_{1} \hat{s}_{3} \hat{s}_{4}\right\rangle_{c}\left\langle\hat{s}_{2}\right\rangle+C^{(2)}
\end{aligned}
$$

where $C^{(2)}$ contains only terms including two-site and on-site terms. Even if there were no correlations between four sites $\left\langle\hat{s}_{1} \hat{s}_{2} \hat{s}_{3} \hat{s}_{4}\right\rangle_{c}=0$, we still need to include the non-vanishing three-site cumulants. Therefore $\left\langle\hat{s}_{1} \hat{s}_{2} \hat{s}_{3} \hat{s}_{4}\right\rangle$ cannot be expressed via two-site and on-site terms. The argument can easily be extended to longer strings and we come to the previously mentioned conclusion.

In general, every string-type correlator can be expressed as a sum of products of 


\section{Non-local correlations in one dimension}

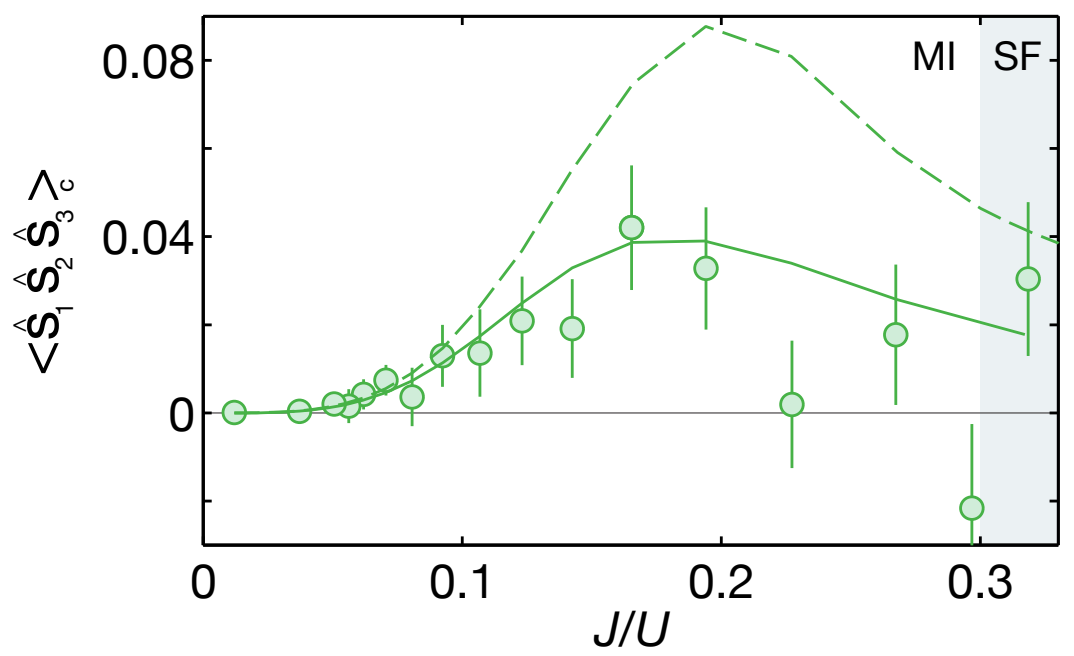

Figure 6.6.: Three-site cumulant. Our experimental values for three site cumulant $\left\langle\hat{s}_{1} \hat{s}_{2} \hat{s}_{3}\right\rangle_{c}$ (green circles) show the existence of three-site correlations in our system. The curves are DMRG calculations for a homogeneous system at $T=0$ (dashed line) and finite-temperature MPS calculations including harmonic confinement at $T=0.09 U / k_{B}$ (solid line).

cumulants similar to Eq. 6.26. Since cumulants are a measure of multi-site correlations, such an expansion gives us information about the contribution of multi-site correlations to string order. In the atomic limit where $J / U \approx 0$, even two-site correlations are absent, but string order is present. In this case, the string signal is trivially dominated by on-site terms. For small but finite $J / U$ values, three-site correlations almost vanish and the string signal is dominated by two-site correlations and on-site terms. In the range of $J / U \approx 0.1-0.2$, three-site correlations build up and also contribute to the string-order signal.

Generally, it would be interesting to know how this relation extends when approaching the critical point. One particularly interesting question is whether multi-site correlations between an infinite number of sites exist in the thermodynamic limit close to the critical point. Such an analysis is difficult both theoretically and experimentally, which can already be seen from the expression for the four-site cumulant. This is however beyond the scope of this thesis and a matter of further investigation.

Instead, we continue with an interpretation of the present three-site correlations. One explanation for such a signal would be the existence of particle-hole pairs extending over three sites. However, another possibility can explain such correlations. Assume we restrict ourselves to a system of only three sites, where the parity on each site can take the values $s_{i}= \pm 1, i=1,2,3$. The system is fully characterized by the probability $p\left(s_{1}, s_{2}, s_{3}\right)$ of finding the parities $s_{1}, s_{2}$ and $s_{3}$ on sites 1,2 and 3 . In a situation, where only nearest-neighbor pairs exist with probability $p_{n n}$, we have $p(+,+,+)=1-2 p_{n n}, p(-,-,+)=p(+,-,-)=p_{n n}$ and 
$p\left(s_{1}, s_{2}, s_{3}\right)=0$ for all other cases. Interestingly, we find a non-vanishing three-site cumulant $\left\langle\hat{s}_{1} \hat{s}_{2} \hat{s}_{3}\right\rangle_{c}=16 p_{n n}^{2}-32 p_{n n}^{3}$ in this situation. The three-site correlation remains present even if we consider the three sites as a subsystem of a longer chain. We conclude that a three-site correlation can arise from next-neighbor pairs alone, simply because site 1 is correlated with site 2 and site 2 is correlated with site 3 . However, our signal extends far beyond the region where first order perturbation theory is valid and we can therefore assume that particle-hole pairs with an extension of more than two sites as well as more complicated clusters play a significant role in our situation.

Finally, we would like to point out an important difference between two-site correlators and string correlators. Averaging over many experimental realizations of the system, a two-site correlator at distance $d$ sums up correlations from pairs with exactly an extension of $d$ but also anti-correlations from all pairs with a different size. In contrast, a string-type correlator of length $l$ sums up positive contributions from all pairs which have a size less than $l$ and lie within the string length. This feature makes the string-type correlator more suitable to study the Mott-insulating phase. In particular, the phase transition in one dimension is marked by the vanishing of the string correlators for long lengths. It is not possible to extract the same information using only two-site correlators. 


\section{Non-local correlations in one dimension}

\subsection{Summary and conclusion}

We presented a theoretical analysis which showed that bosonic Mott Insulators in one dimension possess non-local string order and demonstrated that such non-local correlations can be experimentally measured using single-site-resolved imaging in optical lattices. To the best of the author's knowledge, this has been the first time that correlation functions of this type have been determined experimentally.

Furthermore, our data showed genuine three-site correlations in the form of a three-site cumulant. Further studies could focus on the general role of such multi-site correlations and their behavior close to the critical point. Another open issue is a quantitative theoretical study on the influence of finite temperature.

A future experiment could attempt to measure non-local order in more homogeneous systems at a lower temperatures in order to observe the genuine scaling predicted by zero-temperature theory.

Further, one of the fundamental questions is whether the concepts presented in this chapter have an extension to systems in more than one dimension. For the two-dimensional case, we will present an analysis in the following chapter. 


\section{Non-local correlations in two dimensions, duality and distribution functions}

Non-local order parameters are an important concept for the classification of phases in one dimension (Sec. 6). Here we investigate if a generalization of this concept exists for two dimensions. In general, we are interested in correlation functions of the type

$$
\mathcal{O}^{2}(A)=\left\langle\prod_{i \in A} \hat{u}_{i}\right\rangle
$$

where the product runs over all sites in an area $A$ and $\hat{u}_{i}$ is a local operator. For one-dimensional systems, $A$ is a string of sites, whereas in two-dimensional systems, $A$ is a connected area. The definition of $\mathcal{O}^{2}(A)$ is a direct generalization of the non-local correlation functions for the Bose-Hubbard (Eq. 6.9) and the Ising model (Eq. 6.8) in one dimension.

As in the one-dimensional case, we will start our investigation with a strong-coupling expansion in the Mott-insulating phase (Sec.7.1). The important outcome is that $\mathcal{O}^{2}(A)$ always decays to zero with increasing $A$, in contrast to the one-dimensional case. Therefore, we are forced to analyze the scaling of $\mathcal{O}^{2}(A)$ with $A$, and to investigate possibly different scaling laws in the superfluid and Mott-insulating phases.

We will see that duality transformations provide a framework to understand and calculate such scalings (Sec. 7.2). Our investigation of duality transformations starts with the quantum transverse-field Ising model, where the analysis is more straight forward, but most concepts and results are similar to the Bose-Hubbard model. For both models, we will find that non-local correlations (Eq. 7.1) in two dimension are given by Wilson loop-type correlation functions in dual models. The different scaling of these loop correlation functions distinguishes strong and weak-coupling phases. In the last part of this chapter (Sec. 7.3), we will draw a connection between non-local order parameters and probability distribution functions. One of the important outcomes is that the different scaling of non-local correlations in strong and weakcoupling phases is related to the different scaling of number fluctuations in a certain area. 


\subsection{Intuitive picture for non-local correlations in the two-dimensional Bose-Hubbard model}

For the following discussion, let us define an operator

$$
\hat{C}(A)=\prod_{j \in A} \hat{s}_{j}=\prod_{j \in A} e^{i \pi\left(\hat{n}_{j}-\bar{n}\right)},
$$

which yields a non-local correlation function

$$
\mathcal{O}^{2}(A)=\langle\hat{C}(A)\rangle \text {. }
$$

Our discussion is again based on the strong-coupling expansion from Eq.6.13. As in the one-dimensional case (Sec. 6.2.1), particle-hole pairs reduce $\mathcal{O}^{2}(A)$ only if they are cut at the border of the evaluation area $A$. However, the consequences are different in two dimensions because the border $\partial A$ of the evaluation area grows with $A$. In two dimensions, a large number of particle-hole pairs can be cut if $A$ is large even for small $J / U$. Consequently, we will find that $\mathcal{O}^{2}(A)$ falls off to zero with $A$ for any non-zero $J / U$ (Fig. 7.1).

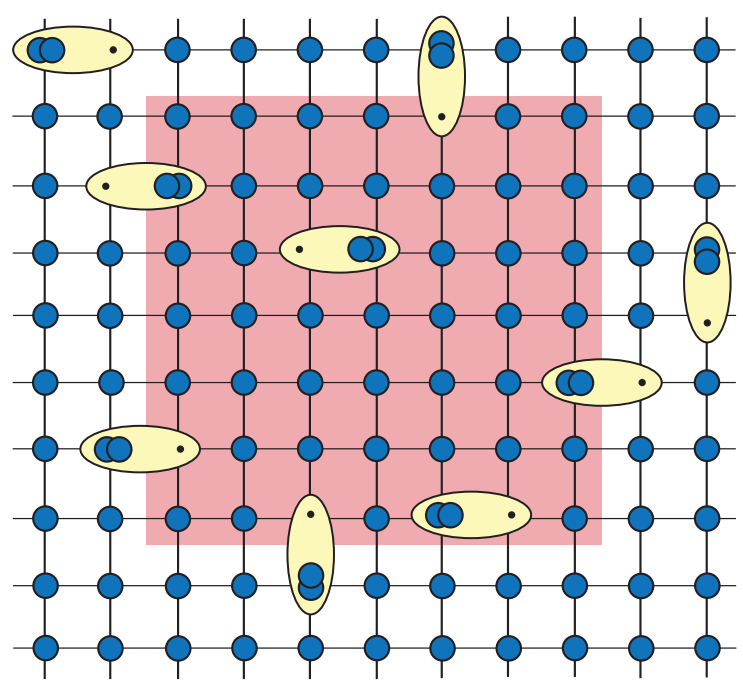

Figure 7.1.: Illustration of non-local correlations in two dimensions. Illustration of a snapshot of the density distribution of a two-dimensional Mott insulator at small, non-zero $J / U$. The evaluation area $A$ for a non-local correlation function $\mathcal{O}^{2}(A)=\left\langle\prod_{i \in A} \hat{s}_{i}\right\rangle$ is sketched with a red shading. Particle-hole pairs reduce $\mathcal{O}^{2}(A)$ if they are cut at the boundary $\partial A$ of $A$. In contrast to one dimension, the boundary $\partial A$ grows with $A$. Therefore, even for a small probability to locally observe a particle-hole pair, a large number of pairs are cut if $\mathcal{O}^{2}(A)$ is evaluated on a large area. As a result, one expects $\mathcal{O}^{2}(A)$ to be a decreasing function of the perimeter size and to eventually fall off to zero. 
We will now give a derivation of this result. First, two cut pairs contribute with two minus signs and cancel each other. In general, for a state where an even number of pairs is cut, $\hat{C}(A)$ has an eigenvalue +1 , while an odd number of pairs yields -1 . We define the quantum mechanical probabilities to cut an even (odd) number of pairs at the boundary of $A$ as $p_{e}(A)\left(p_{o}(A)\right)$. With this, we can write

$$
\mathcal{O}^{2}(A)=p_{e}(A)-p_{o}(A) \text {. }
$$

On each lattice site along the boundary $\partial A$ of $A$, we have a certain local probability $p_{p h}$ to find a particle-hole pair. One can imagine going around the boundary of $A$ and flipping a coin for each site that yields head with probability $p_{p h}$, and in this case we find a particle-hole pair. Repeating this for each site on $\partial A$ resembles a Bernoulli trial of length $\partial A$. The probability to have a total number of successes in this trial is given by a binomial distribution. Therefore, the probability of finding a total number $N_{c}$ of cut particle-hole pairs on all sites of $\partial A$ together is given by a binomial distribution $p_{b}\left(N_{c}, p_{p h}, \partial A\right)$.

The total probabilities $p_{e}(A)$ and $p_{o}(A)$ that $N_{c}$ is even or odd are now simply given by $p_{e}(A)=\sum_{N_{c}, \text { even }} p_{b}\left(N_{c}, p_{p h}, \partial A\right)$ or $p_{o}(A)=\sum_{N_{c}, \text { odd }} p_{b}\left(N_{c}, p_{p h}, \partial A\right)$, where the sums run over all $N_{c}$ that are even or odd, respectively. For a binomial distribution, one finds the exact identity

$$
\sum_{N_{c}, \text { even }} p_{b}\left(N_{c}, p_{p h}, \partial A\right)-\sum_{N_{c}, \text { odd }} p_{b}\left(N_{c}, p_{p h}, \partial A\right)=\left(1-2 p_{p h}\right)^{\partial A},
$$

and therefore

$$
\mathcal{O}^{2}(A) \approx\left(1-2 p_{p h}\right)^{\partial A} .
$$

From the strong coupling expansion to first order in $J / U$ (discussion below Eq.6.13), we know that $p_{p h}=4(J / U)^{2}$. The result in Eq.7.6 coincides with the onedimensional case if we set $\partial A=2$. This yields $\mathcal{O}^{2}(A) \approx 1-16(J / U)^{2}$, up to quadratic order in $J / U$.

In two dimensions, one has to be more careful since high orders of $J / U$ are introduced in Eq.7.6 if $\partial A$ becomes large. Therefore, it is more appropriate to think of Eq. 6.13 as an expansion of the logarithm of the correlation function up to quadratic order in $J / U$, given by

$$
\begin{aligned}
\log \left(\mathcal{O}^{2}(A)\right) & \approx \partial A \cdot \log \left(1-8(J / U)^{2}\right) \\
& \approx-8 \partial A(J / U)^{2} .
\end{aligned}
$$

Importantly, both results yield $\lim _{A \rightarrow \infty} \mathcal{O}^{2}(A)=0$ with a decay that is exponential in the perimeter $\partial A$.

The situation is different in a noninteracting system, where we expect a factorization 


\section{Non-local correlations in two dimensions, duality and distribution functions}

$\mathcal{O}^{2}(A)=\prod_{i \epsilon A}\left\langle\hat{s}_{i}\right\rangle$ (see discussion below Eq. 6.13). As a consequence, we find an exponential decay with the full area $A$. However, as in the one-dimensional case, interactions alter this result (Ch. 7.2.2).

\subsection{Non-local correlations and duality}

In this section, we draw a connection between non-local order and duality transformations. Duality transformations have the property that for quantum mechanical models, a strong-coupling phase is mapped onto a weak-coupling phase and vice versa, and that for classical models, a high-temperature phase is mapped onto a low-temperature phase and vice versa [118-122].

For the models studied here, nearest-neighbor couplings in the original Hamiltonian are reduced to on-site terms in the dual model. At the same time, non-local correlation functions take on a simpler form in the dual representation. Our main result will be that a non-local correlation function, which is defined by a product over an area $A$, reduces to a correlation function, where the product runs only over the border $\partial A$ in the dual description. In one-dimensional systems, this yields simple two-point correlation functions. In two dimensions, we will find correlation functions involving a loop over the perimeter of $A$.

These results are also found for the quantum Ising model in a transverse field. In this case, all relations are exact, whereas in the Bose-Hubbard model approximations are necessary. The Ising model serves two purposes here. First, it is more easily accessible and second, it yields an exact statement that a phase transition can be detected in the scaling of a non-local correlation function with the form of Eq.7.1.

Our presentation of the dual transformations itself, in some parts, follows Refs. [122124]. Similar transformations for the case of the Bose-Hubbard model are possible using a path integral approach [125-127] yielding the same results for non-local correlations in the Bose-Hubbard model [115] as obtained in the following sections.

\subsubsection{Quantum Ising model in a transverse field}

The Hamiltonian $\hat{H}_{I}$ of the quantum Ising model in a transverse field model is defined as [128]

$$
\hat{H}_{I}=-J \sum_{\langle i, j\rangle} \hat{S}_{i}^{z} \hat{S}_{j}^{z}-B \sum_{i} \hat{S}_{i}^{x},
$$

where $J$ the magnetic coupling between neighboring spins and $B$ is the transverse magnetic field. The operators $\hat{S}_{i}^{z}$ and $\hat{S}_{i}^{x}$ are projections of spin-1/2 operators at site $i$ onto the $\mathrm{z}$ and $\mathrm{x}$-axis, respectively. Here we study the ferromagnetic case $(J>0)$. The 
model, in this case, shows a quantum phase transition from a disordered, paramagnetic phase for $B / J>(B / J)_{c}$ to an ordered, ferromagnetic phase for $(B / J)<(B / J)_{c}$.

\section{One-dimensional case}

In a one-dimensional system, one finds $(B / J)_{c}=1$ which can be directly seen using a dual transformation. In the ferromagnetic phase, we find long-range or$\operatorname{der} \lim _{|i-j| \rightarrow \infty}=\left\langle\hat{S}_{i}^{z} \hat{S}_{j}^{z}\right\rangle>0$ in a two-point correlation function. In contrast, the paramagnetic phase has long-range order based on a multi-site correlation function $\lim _{|i-j| \rightarrow \infty}\left\langle\prod_{i<m<j} \hat{S}_{m}^{x}\right\rangle>0$ as we will now show.

The duality transformation is given by $[122,123]$

$$
\begin{aligned}
\hat{S}_{i}^{z} \hat{S}_{i+1}^{z} & =\hat{\sigma}_{i}^{x} \\
\hat{S}_{i}^{x} & =\hat{\sigma}_{i}^{z} \hat{\sigma}_{i+1}^{z} .
\end{aligned}
$$

One can check that the new dual operators $\hat{\sigma}_{i}^{x}$ and $\hat{\sigma}_{i}^{z}$ show the standard commutation relations of spin- $1 / 2$ operators. Furthermore, we can think of the operators $\hat{\sigma}_{i}^{x}$ and $\hat{\sigma}_{i}^{z}$ as sitting on the bonds between the lattice sites $i$ and $i+1$ (Fig. 7.2). The operator $\hat{\sigma}_{i}^{x}$ then captures the coupling between $\hat{S}_{i}^{z}$ and $\hat{S}_{i+1}^{z}$, which results from the first term in the Hamiltonian.

Alternatively, one can imagine the original spin operators sitting on the bonds between the dual spins. In this sense, $\hat{S}_{i}^{x}$ captures the coupling between the dual operators $\hat{\sigma}_{i}^{z}$ and $\hat{\sigma}_{i+1}^{z}$.

The above definitions can be inverted by multiplying strings of operators on both sites. For example, one finds $\hat{\sigma}_{i}^{z}=\prod_{i \leq m} \hat{S}_{m}^{x}$. With this, we have the important relation

$$
\prod_{i \leq m \leq j} \hat{S}_{m}^{x}=\hat{\sigma}_{i}^{z} \hat{\sigma}_{j}^{z}
$$

As a consequence, the non-local correlation function $\left\langle\prod_{i<m<j} \hat{S}_{m}^{x}\right\rangle$ maps onto a twopoint correlation function $\left\langle\hat{\sigma}_{i}^{z} \hat{\sigma}_{j}^{z}\right\rangle$ in the dual language.

We can express the Hamiltonian $\hat{H}_{I}$ in the new variables as

$$
\hat{H}_{I}=-B \sum_{i} \hat{\sigma}_{i}^{z} \hat{\sigma}_{i+1}^{z}-J \sum_{i} \hat{\sigma}_{i}^{x} .
$$

This has the same form as before, but $J$ and $B$ are exchanged, i.e. the quantum Ising model in one dimension is self-dual $[118,119,122]$. Because of the self-duality, the critical value must be $(J / B)_{c}=1$.

In the parameter regime where the original model showed paramagnetism 


\section{Non-local correlations in two dimensions, duality and distribution functions}

$(J<B)$, the dual model shows ferromagnetism and thus long-range order $\lim _{|i-j| \rightarrow \infty}\left\langle\hat{\sigma}_{i}^{z} \hat{\sigma}_{j}^{z}\right\rangle>0$. We can now express $\hat{\sigma}_{i}^{z} \hat{\sigma}_{j}^{z}$ with the original operators using Eq.7.12 and find

$$
\lim _{|i-j| \rightarrow \infty}\left\langle\hat{\sigma}_{i}^{z} \hat{\sigma}_{j}^{z}\right\rangle=\lim _{|i-j| \rightarrow \infty}\left\langle\prod_{i<m<j} \hat{S}_{m}^{x}\right\rangle>0,
$$

in the paramagnetic phase of the original model. This is the crucial result of this subsection. The paramagnetic (disordered) phase is characterized by long-range order in a string correlation function $\left\langle\prod_{i<m<j} \hat{S}_{m}^{x}\right\rangle$. In the dual description, this corresponds to long-range order in a simple two-point correlation function $\left\langle\hat{\sigma}_{i}^{z} \hat{\sigma}_{j}^{z}\right\rangle$.

\section{Two-dimensional case}

Generalizing the previous result, we would like to know if the paramagnetic phase in the two-dimensional model is characterized by a correlation function

$$
\mathcal{O}_{S}^{2}(A)=\left\langle\prod_{i \in A} \hat{S}_{i}^{x}\right\rangle,
$$

where $A$ is a connected area. The answer can, again, be given using a dual transformation. The dual operators are placed on the bonds of a dual lattice, which is obtained by shifting the original lattice by half a lattice constant horizontally and vertically (Fig. 7.2). We will label the sites on the orginal lattice with letters $i, j, \ldots$ and the sites on the dual lattice with $a, b, \ldots$.

To each site on the dual lattice, one assigns spin operators $\hat{\sigma}_{a, 1}^{x}, \hat{\sigma}_{a, 1}^{z}\left(\hat{\sigma}_{a, 2}^{x}, \hat{\sigma}_{a, 2}^{z}\right)$ on the bonds to the nearest-neighbors in horizontal (vertical) direction, respectively (Fig. 7.2). By doing so one doubles the number of operators compared to the original model.

Alternatively, one can view the dual variables to sit on the bonds of the original lattice. Similar to the one-dimensional case, we would like to capture the nearest neighbor coupling of $\hat{S}_{i}^{z}$ in a dual spin operator $\sigma_{a, \alpha}^{x}$ sitting on the bond between neighboring sites. This is accomplished by the dual transformation [122, 123]

$$
\begin{aligned}
& \hat{\sigma}_{a, 1}^{x}=\hat{S}_{i}^{z} \hat{S}_{i+e_{2}}^{z} \\
& \hat{\sigma}_{a, 2}^{x}=\hat{S}_{i}^{z} \hat{S}_{i+e_{1}}^{z}
\end{aligned}
$$

where $e_{1}$ and $e_{2}$ are the unit vectors in $\mathrm{x}$ and $\mathrm{y}$-directions, respectively. The spins on the right hand side correspond to the original spins connected to the bond, where $\hat{\sigma}_{a, 1}^{x}$ $\left(\hat{\sigma}_{a, 2}^{x}\right)$ is defined (Fig. 7.2b). We will turn to the dual transformation for $\hat{S}_{i}^{x}$ in Eq. 7.19. The same original spin $S_{i}^{z}$ is involved in the definition of four different spins in the 
$1 d$

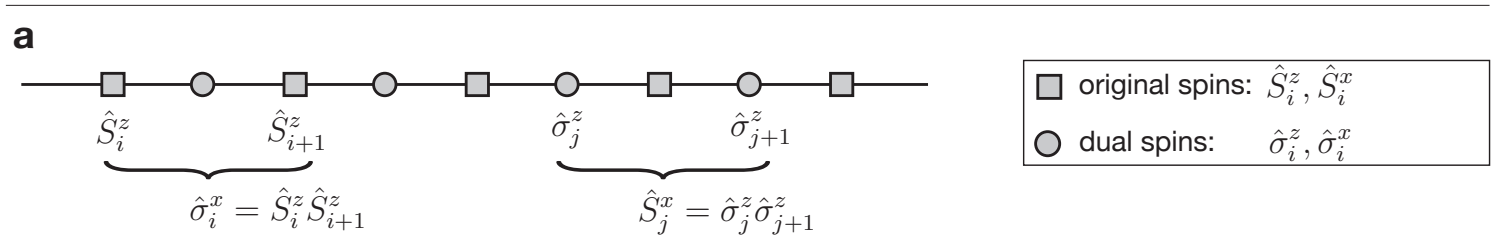

$2 d$
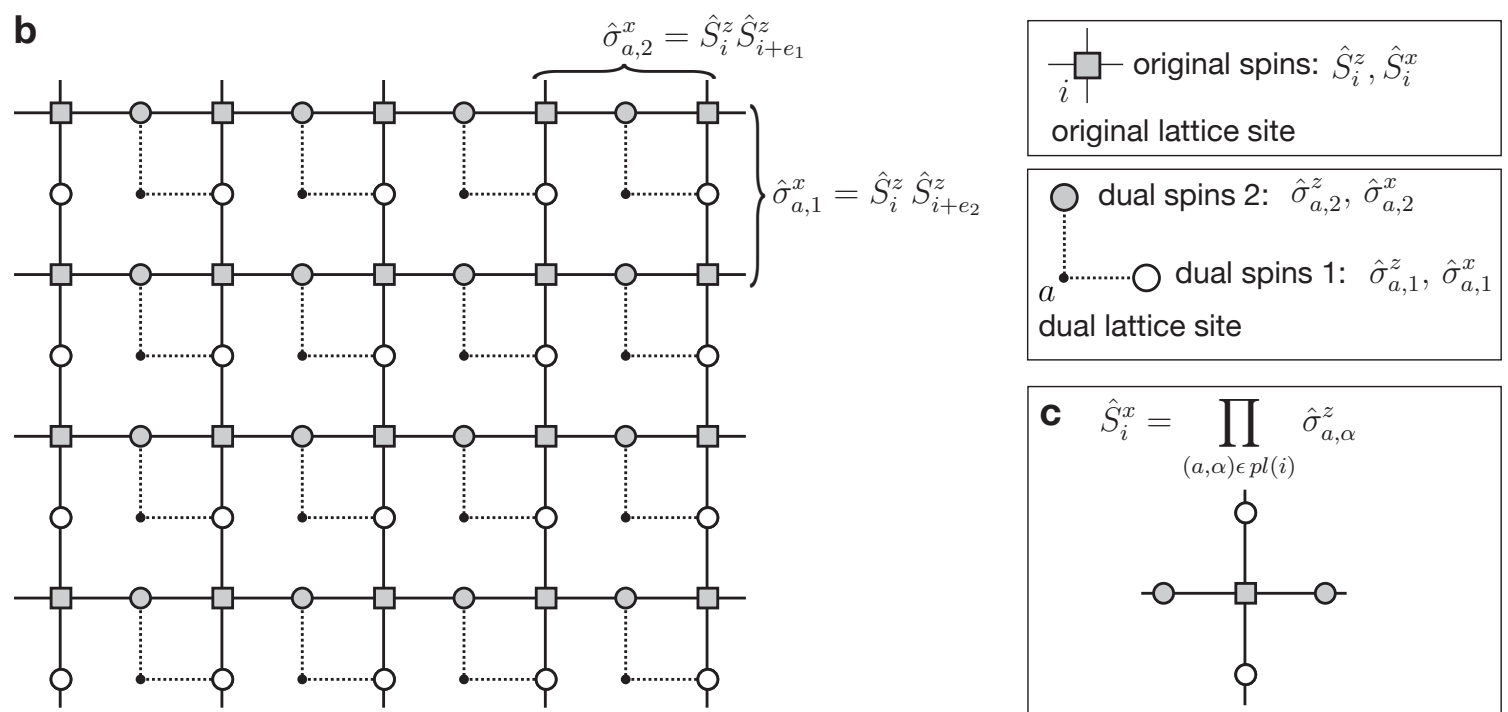

Figure 7.2.: Dual transformation of the Ising model . a, Illustration of the dual transformation for the one-dimensional Ising model. The dual spin operators $\sigma_{i}^{x}, \sigma_{i}^{z}$ sit on the bonds of the original lattice. $\mathbf{b}$, Illustration of the dual transformation for the two-dimensional Ising model. The dual lattice is obtained by shifting the original lattice by half a lattice constant horizontally and vertically (black dots). At each dual lattice site, dual operators are placed on the vertical and horizontal bond to the nearest neighbor (dashed lines and connected circles). c, Illustration of the definition of $\hat{S}_{i}^{x}$ that involves a product of all dual spins on a plaquette surrounding the original lattice site $i$.

dual model, sitting on all bonds emanating from $i$. For this reason, not all dual operators $\hat{\sigma}_{a, \alpha}^{x}$ can be independent. One can check that this leads to a restriction of the form [123]

$$
1=\prod_{(b, \beta) \epsilon i} \hat{\sigma}_{b, \beta^{\prime}}^{x}
$$

where $(b, \beta) \epsilon i$ refers to all spins on the dual lattice that sit on the bonds emanating from site $i$ of the original lattice. The condition restricts the allowed values for the eigenvalues of $\hat{\sigma}_{b, \beta}^{x}$ and the Hilbert space of the dual theory has to be limited in this way. 


\section{Non-local correlations in two dimensions, duality and distribution functions}

The dual transformation involving $\hat{S}_{i}^{x}$ in the one-dimensional case was such that $\hat{S}_{i}^{x}$ captured the coupling of dual spin operators $\hat{\sigma}_{j}^{z}$ on neighboring sites. In the twodimensional geometry this can not be trivially achieved, since $\hat{S}_{i}^{x}$ is surrounded by four dual spins and not only by two. To solve the problem, one choses a dual transformation for $\hat{S}_{i}^{x}$ given by $[122,123]$

$$
\hat{S}_{i}^{x}=\prod_{(a, \alpha) \in p l(i)} \hat{\sigma}_{a, \alpha}^{z}
$$

where the rule $(a, \alpha) \in \mathrm{pl}(i)$ refers to all dual spins surrounding site $i$ of the original lattice (see Fig. 7.2c).

With these transformations the Hamiltonian can be written as

$$
\hat{H}_{I}=-B \sum_{i} \prod_{(a, \alpha) \epsilon p l(i)} \hat{\sigma}_{a, \alpha}^{z}-J \sum_{a, \alpha} \hat{\sigma}_{a, \alpha}^{x} .
$$

Note that now the coupling term involves four instead of two spins and the model is, therefore, of different form than the original Hamiltonian.

The new form of the Hamiltonian, as well as the rules for the dual transformation, are invariant under a local transformation

$$
\hat{\sigma}_{a, \alpha}^{z} \rightarrow-\hat{\sigma}_{a, \alpha}^{z} \forall(a, \alpha) \in p l(b) .
$$

The transformation describes flipping all spins on a plaquette surrounding a site of the dual lattice. Therefore, we are dealing with a theory that has a $Z_{2}$ gauge invariance. The model is usually referred to as Ising lattice gauge theory [122].

Our general interest is in the transformation of the observable $\mathcal{O}^{2}(A)$. Before dealing with this, it is instructive to discuss some general facts about correlation functions in the dual theory. The dual model Hamiltonian must show a phase transition, since we know that the original model does. This phase transition should be detectable in the behavior of a correlation function. In particular, for large $B$ values, one would expect an ordering in a two point correlation function based on $\sigma_{a, \alpha}^{z}$. However, any such two-point correlator involving spins that are far apart is not gauge invariant and consequently equal to zero [122].

To solve the problem, Wegner introduced so call loop correlation functions that are gauge invariant $[122,129]$. The concept was later generalized by Wilson in the general context of lattice gauge theories, and the corresponding correlation functions are commonly known as Wilson loops [130].

For the model above, we define a loop correlation function

$$
W(A)=\left\langle\prod_{(i, a) \epsilon \partial A} \hat{\sigma}_{i, a}^{z}\right\rangle,
$$


where the product runs over the closed loop given by the perimeter $\partial A$ of the Area $A$ (Fig. 7.3). We will see shortly that this quantity is indeed gauge invariant.

The two phases are now characterized by a different scaling of the Wilson loop with the size of the area $A$. One finds an exponential decay with the area $W(A) \propto e^{-A}$ in a parameter regime for which the original model is in the ferromagnetic phase, and, in contrast, a decay with the perimeter $W(A) \propto e^{-\partial A}$ in the original paramagnetic phase (see, e.g, Ref. [121] and references therein). In the previous expressions, we omitted numerical constants and only showed the scaling behavior.

A natural question is if the Wilson-loop correlation function can be expressed using the spin operators of the original theory. The simplest loop is formed by four sites of the dual lattice that form a plaquette which encloses a site $i$ of the orignal lattice. The loop is then simply given by the expectation value $\left\langle\hat{S}_{i}^{x}\right\rangle$, because of the definition of the dual transformation in Eq.7.19. Extending the loop by two more sites of the dual lattice, one finds a loop around two neighboring spins $i, j$ of the original lattice. The loop correlation function is then simply expressed as the product $\left\langle\hat{S}_{i}^{x} \hat{S}_{j}^{x}\right\rangle$ because the single dual spin $\hat{\sigma}_{a, \alpha}^{z}$, shared by these two original spins, is canceled. Generalizing this, one finds via induction that

$$
W(A)=\mathcal{O}_{S}^{2}(A)=\left\langle\prod_{i \in A} \hat{S}_{i}^{x}\right\rangle,
$$

which forms the central result of this subsection. The correlation function formed by a product of all $S_{i}^{x}$ in an area $A$ is given by a Wilson-loop over the border $\partial A$ in the dual description (Fig. 7.3). This result also implicitly proves that the Wilson loop correlation function is gauge invariant, because $\hat{S}_{i}^{x}$ is gauge invariant.

From the relation above, we know the scaling of $\mathcal{O}_{S}^{2}(A)$ in both phases. We find that $\mathcal{O}_{S}^{2}(A)$ scales exponentially with the perimeter of $A$ in the paramagnetic phase and with the full area in the ferromagnetic case. The paramagnetic phase is, therefore, characterized by a slower decay of $\mathcal{O}_{s}^{2}(A)$. However, in both phases $\mathcal{O}_{S}^{2}(A)$ decays to zero. Our argumentation is a proof of principle that a phase transition in a twodimensional system can be detected using a non-local correlation function with the general form of $\mathcal{O}^{2}(A)$ defined in Eq. 7.1.

\subsubsection{Bose-Hubbard model}

We now turn to the connection between non-local order and dual transformations in the Bose-Hubbard model. The relation is, so far, only known for the quantum rotor model [18], defined as

$$
\hat{H}_{\text {rotor }}=-E_{J} \sum_{\langle i, k\rangle} \cos \left(\hat{\phi}_{i}-\hat{\phi}_{j}\right)+U \sum_{i}\left(\hat{n}_{i}-\bar{n}\right)^{2},
$$




\section{Non-local correlations in two dimensions, duality and distribution functions}

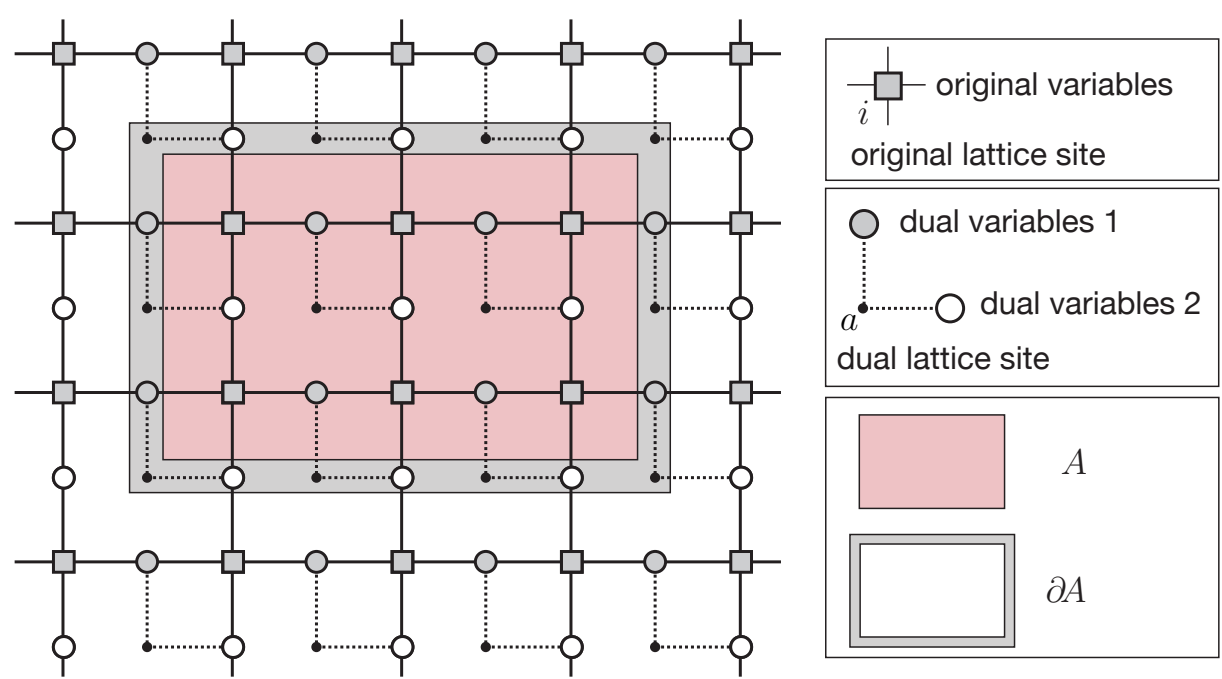

Figure 7.3.: Loop and area correlations. Illustration of loop and area correlation functions. A correlation function that involves an area $A$ (red shading) in the original description is transformed into a correlation function involving only the boundary $\partial A$ (grey shading) in the dual description.

where $E_{J}=2 \bar{n} J$. The quantum rotor model describes the physics of the BoseHubbard model quantitatively for large average occupation numbers $\bar{n}$. The relation to operators in the Bose-Hubbard model is $\hat{b}_{i}=\sqrt{\hat{n}} e^{-i \hat{\phi}}\left(\hat{b}_{i}^{\dagger}=\sqrt{\hat{n}} e^{i \hat{\phi}}\right)$. The density $\hat{n}_{i}$ and phase $\hat{\phi}_{i}$ operators obey canonical commutation relations. For a discussion of possible methods for low average occupation numbers, see the conclusion section 7.4.

\section{One-dimensional case}

The dual transformation in the one-dimensional case is defined by $[123,124]$

$$
\begin{aligned}
& \hat{E}_{i}=\frac{1}{2 \pi}\left(\hat{\phi}_{i+1}-\hat{\phi}_{i}\right) \\
& \hat{n}_{i}=\frac{1}{2 \pi}\left(\hat{\theta}_{i+1}-\hat{\theta}_{i}\right)+\bar{n} .
\end{aligned}
$$

As for the Ising model, the new dual variable $\hat{E}_{i}$ captures the nearest-neighbor coupling of the original model. The original variable $\hat{n}_{i}$ captures the coupling of the dual variable $\hat{\theta}_{i}$, where we additionally added the average density $\bar{n}$. The operator $\hat{\theta}_{i}$ has $2 \pi$-spaced discrete eigenvalues and the new set of operators $\hat{\theta}_{i}$ and $\hat{E}_{i}$ have canonical commutation relations.

The Hamiltonian reads

$$
\hat{H}_{\text {rotor }}=-E_{J} \sum_{i} \cos \left(2 \pi \hat{E}_{i}\right)+\frac{U}{(2 \pi)^{2}} \sum_{i}\left(\hat{\theta}_{i+1}-\hat{\theta}_{i}\right)^{2}
$$


in terms of the dual operators and is complicated to handle because of the integer constraint for $\hat{\theta}_{i}$. One can show that this constraint can be relaxed by replacing

$$
-E_{J} \cos \left(2 \pi \hat{E}_{i}\right) \rightarrow \frac{1}{2 \kappa} \hat{E}_{i}^{2}-\tilde{t} \cos \left(\hat{\theta}_{i}\right)
$$

where the cosine-term on the right-hand side favors integer eigenvalues energetically. We refer to Ref. [124] for a detailed discussion of this replacement. The new variables $\kappa$ and $\tilde{t}$ have to be fixed by a comparison with numerical calculations, similar to the pre-factors in the sine-Gordon Hamiltonian (Eq. 6.20).

With this, we find for the dual Hamiltonian

$$
\hat{H}_{\text {rotor }} \approx \frac{1}{2 \kappa} \sum_{i} \hat{E}_{i}^{2}+\frac{U}{(2 \pi)^{2}} \sum_{i}\left(\hat{\theta}_{i+1}-\hat{\theta}_{i}\right)^{2}-\tilde{t} \cos \left(\hat{\theta}_{i}\right) .
$$

which is a discrete version of the sine-Gordon Hamiltonian previously studied (Eq. 6.20). Indeed, the Bosonization transformation (Sec. 6.16) in the long-wavelength limit takes on the same structure as the dual transformation based on the quantum rotor model presented here. In this sense, one can view Bosonization of the BoseHubbard model as a dual transformation.

In our previous analysis, we have already found that the string order correlation function transforms into a two point correlation function in the dual description (Eq. 6.18) and presented a calculation showing that the Mott phase is characterized by string order (Sec. 6.16 and App.C).

\section{Two-dimensional case}

The extension to the two-dimensional case is similar to the Ising model. We place dual variables on bonds of a dual lattice, whereby we define $\hat{E}_{a, 1}, \hat{A}_{a, 1}$ on horizontal bonds and $\hat{E}_{a, 2}, \hat{A}_{a, 2}$ on vertical bonds (Fig. 7.4).

The dual transformation involving $\hat{\phi}_{i}$ is given by $[123,124]$

$$
\begin{aligned}
& \hat{E}_{a, 1}=\frac{1}{2 \pi}\left(\hat{\phi}_{i+e_{2}}-\hat{\phi}_{i}\right) \\
& \hat{E}_{a, 2}=-\frac{1}{2 \pi}\left(\hat{\phi}_{i+e_{1}}-\hat{\phi}_{i}\right) .
\end{aligned}
$$

The right hand sites include the phase operators connected to the bond on which $\hat{E}_{a, 1}$ $\left(\hat{E}_{a, 2}\right)$ is defined.

As for the two-dimensional Ising model, we need a restriction of the allowed Hilbert space for the dual variables $\hat{E}_{a, \alpha}$. To see this, one can sum up the phase differences around a plaquette on the original lattice. This sum has to be equal to an integer number times $2 \pi$, where the integer value corresponds to the local vorticity of the 


\section{Non-local correlations in two dimensions, duality and distribution functions}

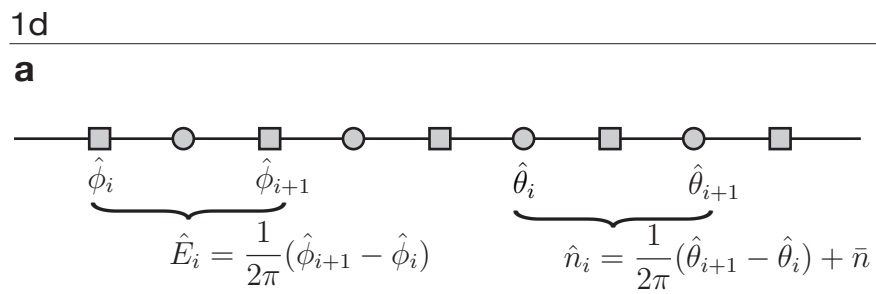

original variables: $\hat{n}_{i}, \hat{\phi}_{i}$

dual variables: $\hat{\theta}_{i}, \hat{E}_{i}$

$2 d$

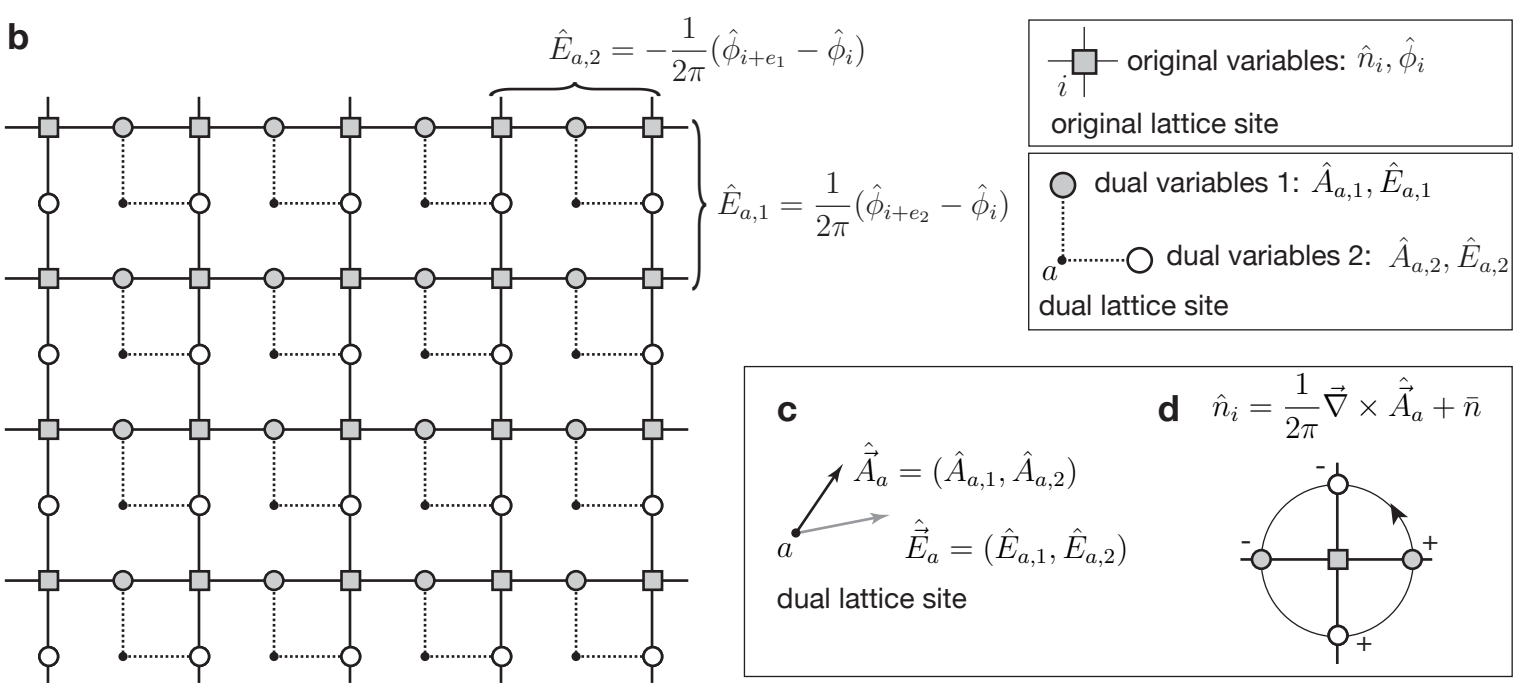

Figure 7.4.: Dual transformation of the quantum rotor model. a, Illustration of the dual transformation of the one-dimensional quantum rotor model. The dual operators $\hat{E}_{i}, \hat{\Theta}_{i}$ sit on the bonds of the original lattice. $\mathbf{b}$, Illustration of the dual transformation of the twodimensional quantum rotor model. The dual lattice is obtained by shifting the original lattice by half a lattice constant horizontally and vertically (black dots). At each dual lattice site, dual operators are placed on the vertical and horizontal bond to the nearest neighbor (dashed lines and connected circles). c, The dual variables can be combined to vectors $\hat{\vec{A}}_{a}$ and $\hat{\vec{E}}_{a}$ that form a two-dimensional vector field. d, Illustration of the dual transformation involving $\hat{\vec{A}}_{a}$, which is defined such that its lattice curl is proportional to the deviation from mean density $\hat{n}_{i}-\bar{n}$. The lattice curl is defined as a summation of the elements of $\hat{\vec{A}}_{a}$ shown in the figure with associated pre-factors (Eq. 7.34).

phase field. Expressed in the dual variables, we find [124]

$$
\vec{\nabla} \cdot \vec{E}_{a} \equiv\left(\hat{E}_{a, 1}-\hat{E}_{a-e_{1}, 1}\right)+\left(\hat{E}_{a, 2}-\hat{E}_{a-e_{2}, 2}\right)=\hat{N}_{a}
$$

where $\hat{N}_{a}$ is an operator for the local vorticity of the phase field at the plaquette of the original lattice which surrounds the site $a$ of the dual lattice. The Hilbert space of the dual theory has to be restricted such that the eigenvalues of $\hat{N}_{a}$ are integers. The equation can be interpreted as a Gauss law for integer charges. To simplify notation, 
we defined a discrete version of the divergence operation on a vector $\hat{\vec{E}}_{a}=\left(\hat{E}_{a, 1}, \hat{E}_{a, 2}\right)$. The dual transformation involving $\hat{n}_{i}$ is given by $[123,124]$

$$
\begin{aligned}
\hat{n}_{i} & =\frac{1}{2 \pi}\left[\left(\hat{A}_{a+e_{1}, 2}-\hat{A}_{a, 2}\right)-\left(\hat{A}_{a+e_{2}, 1}-\hat{A}_{a, 1}\right)\right]+\bar{n} \\
& \equiv \frac{1}{2 \pi} \nabla \times \hat{\vec{A}}_{a}+\bar{n}
\end{aligned}
$$

where $a$ refers to the site on the dual lattice which is closest to the original site $i$ in the left, lower direction of $i$. In the second line, we defined a discrete version of a two-dimensional curl of the vector $\hat{\vec{A}}_{a}=\left(\hat{A}_{a, 1}, \hat{A}_{a, 2}\right)$.

To complete the transformation, we state that one can chose the commutation relations between $\hat{E}_{a, \alpha}$ and $\hat{A}_{a, \alpha}$ to have a canonical form, that is $\hat{E}_{a, \alpha}$ and $\hat{A}_{a, \alpha}$ can be considered as canonical position and momentum operators [124].

For the dual Hamiltonian, we find

$$
\hat{H}_{\text {rotor }}=-E_{J} \sum_{a} \cos \left(2 \pi \hat{\vec{E}}_{a}\right)+\frac{U}{(2 \pi)^{2}} \sum_{a}\left(\nabla \times \hat{\vec{A}}_{a}\right)^{2} .
$$

This Hamiltonian and also the transformation in Eq.7.34 are invariant under an addition of a rotation-free term to $\hat{\vec{A}}_{a}$. We are therefore dealing with a gauge theory. With the same replacement as in the one-dimensional case, to soften the integer constraint, we find

$$
\hat{H}_{\text {rotor }} \approx \frac{1}{2 \kappa} \sum_{a} \hat{\vec{E}}_{a}^{2}+\frac{U}{(2 \pi)^{2}} \sum_{a}\left(\nabla \times \hat{\vec{A}}_{a}\right)^{2}-\tilde{t} \sum_{a, \alpha} \cos \left(\hat{A}_{a, \alpha}\right) .
$$

The complete dual theory is given by this Hamiltonian and the restriction in Eq. 7.32. The first two terms of the dual Hamiltonian have the same form as the conventional Hamiltonian for electric and magnetic fields $\left(\propto \vec{E}^{2}+\vec{B}^{2}\right)$, with the magnetic field expressed as the curl of the vector-potential $\vec{A}$.

In this analogy, the eigenspectrum for $\tilde{t}=0$ is composed of massless photons which are given by the Bogoliubov excitations of the superfluid (App.D). The presence of the last term $(\tilde{t}>0)$ leads to a finite mass of the photons or, equivalently, to a gapped spectrum corresponding to particle and hole-excitations in the Mott-insulating phase. We now turn to the transformation of the non-local correlation function $\mathcal{O}^{2}(A)$ defined in Eq.7.3. With Eq. 7.34, we find

$$
\begin{aligned}
\mathcal{O}^{2}(A) & =\left\langle\prod_{j \in A} e^{i \pi\left(\hat{n}_{j}-\bar{n}\right)}\right\rangle=\left\langle e^{i \pi \sum_{j \epsilon A}\left(\hat{n}_{j}-\bar{n}\right)}\right\rangle \\
& =\left\langle e^{\left.i \frac{1}{2} \sum_{a \epsilon A} \nabla \times \hat{A}_{a}\right)}\right\rangle \\
& =\left\langle e^{i \frac{1}{2} \sum_{(a, \alpha) \epsilon \partial A} \hat{A}_{a, \alpha}}\right\rangle,
\end{aligned}
$$




\section{Non-local correlations in two dimensions, duality and distribution functions}

where in the last line we used a discrete version of Stokes theorem. The sum $(a, \alpha) \epsilon \partial A$ runs over all dual variables crossed by the boundary of $A$ (Fig. 7.3). This forms the central result of this section. The non-local correlation function over an area $A$ is reduced to an expectation value involving a loop around the border of $A$ in the dual theory. The latter can be considered as a Wilson-loop in purely spatial directions.

The calculation using the dual theory is possible in a harmonic approximation of the cosine-terms in the Hamiltonian (App.D). The result is a scaling of the form

$$
\mathcal{O}^{2}(A) \propto e^{-\partial A}
$$

in the Mott phase and

$$
\mathcal{O}^{2}(A) \propto e^{-\partial A \log (\partial A)}
$$

in the superfluid phase, where we omitted constants in the exponential functions. As already anticipated by the strong-coupling argument in Sec. 7.1, $\mathcal{O}^{2}(A)$ falls off to zero also in the Mott-insulating phase. There is no non-local order in a strict sense. However, we find as a main conclusion that the superfluid and the Mott-insulating phases can be distinguished by a different scaling of $\mathcal{O}^{2}(A)$. The distinction is not as sharp as in the two-dimensional Ising case where we found an area vs. a perimeter law.

\subsection{Distribution functions}

In this section, we draw a connection between the non-local order parameter defined in Eq.7.3 and the form of certain probability distribution functions. We will investigate two different cases. First, the probability distribution to observe a certain atom number in a given area (Sec. 7.3.1). Second, the probability to observe a certain number of lattice sites, which have an on-site occupation that shows an odd deviation $\delta n$ from the mean atom number (Sec. 7.3.2).

\subsubsection{Atom number distribution}

The probability distribution to observe a certain atom number in a given area has recently been studied in Refs. [131-135] for several different many-body systems. Let us first explain this concept in some detail. Imagine that experimentally we could count the atom number $N$ in a given area $A$. In a fluctuating many-body system, this quantity will fluctuate in each experimental repetition. Gathering statistics by repeating the experiment, we could measure the probability $p(N, A)$ to find a certain number of atoms $N$ in the area $A$. We can interpret $p(N, A)$ as the quantum mechanical probability to observe an eigenvalue $N$ of the atom number operator $\hat{N}_{A}=\sum_{i \epsilon A} \hat{n}_{i}$ for the 
area $A$.

One important question is if we can distinguish different quantum phases by analyzing $p(N, A)$ as a function of $A$. It turns out that, in many cases, the information about the variance $\sigma^{2}(A)$ of the distribution, defined as

$$
\begin{aligned}
\sigma^{2}(A) & =\left\langle\left(\hat{N}_{A}-\bar{N}_{A}\right)^{2}\right\rangle \\
& =\sum_{N} p(N, A)\left(N-\bar{N}_{A}\right)^{2}
\end{aligned}
$$

is sufficient for this [134]. Here we defined $\bar{N}_{A}=\left\langle\hat{N}_{A}\right\rangle=\sum_{N} p(N, A) N$ and we will only consider commensurate filling, i.e., $\bar{N}_{A}$ is an integer.

The authors of Ref. [134] claim that, in general, $\sigma^{2}(A)$ scales as

$$
\sigma^{2}(A) \propto L^{d-1}
$$

in a gapped phase, and as

$$
\sigma^{2}(A) \propto L^{d-1} \log (L)
$$

in a gapless phase, which is also found by a direct calculation for the Bose-Hubbard model [115]. In both cases, zero temperature is assumed. With $L$ we refer to the linear extension of the area $A$. In one dimension, $L$ is proportional to the string length while in two-dimensions, $L$ is proportion to the circumference $\partial A$ of $A$.

We now draw a connection between this scaling and the previously found scaling for the non-local correlation function (Eq.7.40 and 7.41). First, we can write the non-local order parameter as

$$
\mathcal{O}^{2}(A)=\left\langle e^{i \pi \sum_{j \epsilon A}\left(\hat{n}_{j}-\bar{n}\right)}\right\rangle=\left\langle e^{i \pi\left(\hat{N}_{A}-\bar{N}_{A}\right)}\right\rangle
$$

Within a quadratic approximation of the Hamiltonian, we can simplify this expression to (see, e.g., Refs. [111, 136])

$$
\mathcal{O}^{2}(A) \approx e^{-\frac{\pi^{2}}{2}\left\langle\left(\hat{N}_{A}-\bar{N}_{A}\right)^{2}\right\rangle}=e^{-\frac{\pi^{2}}{2} \sigma(A)^{2}}
$$

Therefore, the scaling of the non-local correlation function is, in this approximation, dominated by the scaling of the the variance of the atom number distribution.

With the results of Eq.7.44 and Eq.7.45, we find $\log \left(\mathcal{O}^{2}(A)\right) \propto-L^{d-1}$ in Mottinsulating phase and $\log \left(\mathcal{O}^{2}(A)\right) \propto-L^{d-1} \log (L)$ in the superfluid phase. This is the same result as in Eq. 7.40 and 7.41 derived for the two-dimensional case via different methods. We also recover $\log \left(\mathcal{O}^{2}(A)\right) \propto$ const. for Mott insulators in one dimension (Ch. 6). 


\section{Non-local correlations in two dimensions, duality and distribution functions}

Without approximation, one can write Eq. 7.46 as

$$
\begin{aligned}
\mathcal{O}^{2}(A)= & \left\langle(-1)^{\left(\hat{N}_{A}-\bar{N}_{A}\right)}\right\rangle=\sum_{N} p(N, A)(-1)^{N-\bar{N}_{A}} \\
= & \sum_{\substack{\left(N-\bar{N}_{A}\right)=\text { even }\\
}} p(N, A)-\sum_{\left(N-\bar{N}_{A}\right)=\text { odd }} p(N, A) \\
= & \underset{\left(N-2 \cdot \sum_{A}\right)=\text { odd }}{1-2} p(N, A)
\end{aligned}
$$

The non-local order parameter $\mathcal{O}^{2}(A)$, therefore, probes an unconventional property of $p(N, A)$; namely, the total probability to observe an atom number $N$ that has an odd difference from the mean atom number $N-\bar{N}_{A}$. We illustrate this in Fig. 7.5.

With this, we can get a better understanding of Eq.7.47. Intuitively, we expect that a narrower distribution $p(N, A)$ leads to a lower total weight for all $N$, for which $N-\bar{N}_{A}$ is odd and, thus, to a finite value of $\mathcal{O}^{2}(A)$ (Fig. 7.5). For an infinitely broad distribution (for an infinite $\bar{N}_{A}$ ), we expect that the probability to observe an odd $N-\bar{N}_{A}$ is exactly $1 / 2$, and, therefore, $\mathcal{O}^{2}(A)$ vanishes. The crucial point for onedimensional Mott insulators is that the width of the distribution $p(N, A)$ stays finite in the limit $A \rightarrow \infty$ (and also $\bar{N}_{A} \rightarrow \infty$ ).

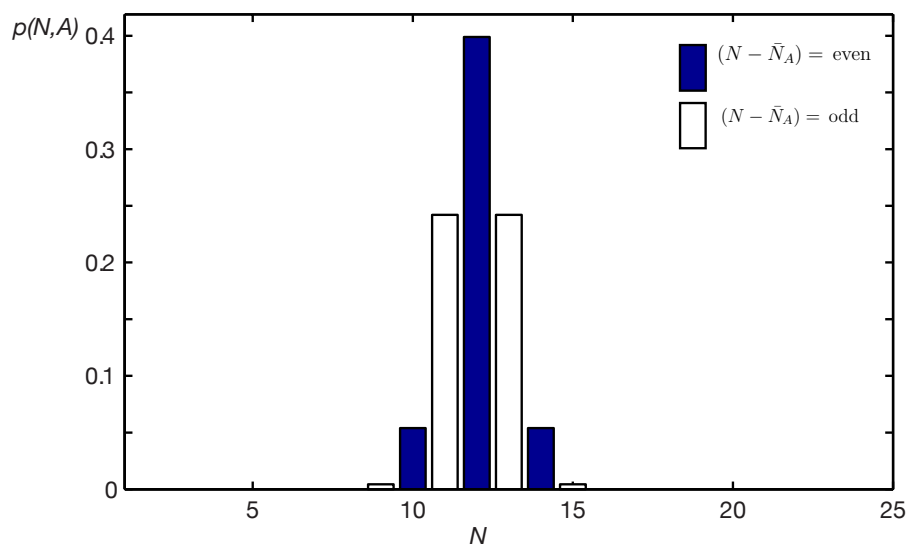

Figure 7.5.: Distribution function $p(N, A)$. Illustration of an atom number distribution function $p(N, A)$ with $\bar{N}_{A}=12$ using a bar chart. The values for which $N-\bar{N}_{A}$ is even (odd) are colored in blue (white). The total probability to find a value of $N$, for which $N-\bar{N}_{A}$ is odd (even) is given by the sum of all blue (white) bars. For a narrow distribution, as the one shown in this figure, the total probability to find a value of $N$ for which $N-\bar{N}_{A}$ is odd is reduced compared to the total probability to observe a value of $N$ for which $N-\bar{N}_{A}$ is even. 


\subsubsection{Parity distribution}

Assume we experimentally determine the on-site parity (Sec.3.4) in a region of interest $A$. Let us define $N_{o}$ as the number of lattice sites in this region for which the local deviation from the mean occupation $n_{i}-\bar{n}$ is odd. For the case of $\bar{n}=1$, we can write $N_{o}$ as $N_{o}=\sum_{i \epsilon A}\left(1-P_{i}\right)$, where $P_{i}=\bmod _{2} n_{i}$ is the observed on-site parity. One can think of $N_{o}$ as the number of defects in the region $A$. Via repeated measurements, we can determine the probability distribution $p_{o}\left(N_{o}, A\right)$ to observe a certain $N_{0}$.

The important observation is that $e^{i \pi \sum_{j e A}\left(\hat{n}_{j}-\bar{n}\right)}$ yields $1(-1)$ if $N_{o}$ is even (odd). With this, we can write

$$
\mathcal{O}^{2}(A)=\left\langle\prod_{j \in A} \hat{s}_{j}\right\rangle=\sum_{N_{o}=\text { even }} p_{o}\left(N_{o}, A\right)-\sum_{N_{o}=\text { odd }} p_{o}\left(N_{o}, A\right) .
$$

The formula states that to evaluate non-local order, one simply has to count the number of sites with an occupation number for which $n_{i}-\bar{n}$ is odd. The physical meaning is that deep in the Mott limit, we have $\hat{n}_{i}-\bar{n}=0$ for almost all sites, and therefore $p\left(N_{o}, A\right) \approx \delta_{N_{0}, 0}$, where $\delta_{i, j}$ is the Kronecker delta. A particle-hole pair within $A$ introduces two sites for which $\hat{n}_{i}-\bar{n}$ is odd, yielding an increased probability to observe an even number $N_{0}$.

Despite the fact that $\mathcal{O}^{2}(A)$ can be calculated from $p(N, A)$ via Eq.7.50 and from $p_{o}\left(N_{o}, A\right)$ via Eq.7.51, one can, unfortunately, not construct $p(N, A)$ from the knowledge of $p_{o}\left(N_{o}, A\right)$.

Finally, the knowledge of $p_{0}\left(N_{0}, A\right)$ for all areas $A$ (including disconnected ones) allows for the calculation of arbitrary correlators $\left\langle\prod_{j \in A} \hat{s}_{j}\right\rangle$ via Eq.7.51. 


\subsection{Summary and conclusion}

In summary, we presented theoretical arguments that different quantum phases in two dimensions can be distinguished by analyzing the scaling of non-local correlation functions. In particular, we found different scaling laws in the strong and weak-coupling phases of the quantum transverse field Ising model (Sec.7.2.1) and of the quantum rotor model (Sec. 7.2.2). Both models form paradigm examples that show a quantum phase transition [20].

One of the open questions is whether the obtained results also apply to different models. This is indicated by the connection between non-local correlation functions and the scaling of number fluctuations (Sec.7.3.1). The latter was recently investigated for various different models and different scalings were found in ordered and disordered phases [134], which also suggests different scalings of non-local correlation functions (Eq.7.47 and 7.50).

Specifically, for the quantum rotor model, we found a scaling for non-local correlations with $\log \left(\mathcal{O}^{2}(A)\right) \propto-L^{d-1}$ in the Mott-insulating phase and $\log \left(\mathcal{O}^{2}(A)\right) \propto-L^{d-1} \log (L)$ in the superfluid phase. With $L$ we refer to the string-length in one dimension $(d=1)$ and to the circumference of the evaluation area in two dimensions $(d=2)$. In one dimension, this leads to string order in the Mott-insulating phase $\left(\mathcal{O}^{2}(A) \propto\right.$ const.) and to an algebraic decay in the superfluid phase. Importantly, the distinction between the scalings for the two phases in two dimensions is as strong as for the one-dimensional case. However, there is no string order, in the strict sense, in the Mott-insulating phase in two dimensions. It is an open question whether the same scalings hold in three dimensions.

We expect these scalings to be correct for the Bose-Hubbard model with large filling. For low fillings, further investigations, e.g., using numerical methods such as QMC, are desirable. At unity filling, it might be possible to find a dual transformation in two dimensions based on a spin-1 model, which has been used as an approximate description of the Bose-Hubbard model (see, e.g., Ref. [99]).

Finally, a future experimental study could aim at the observation of the different scalings in a two-dimensional system. 


\section{Part III.}

'Higgs' amplitude mode 
Spontaneous symmetry breaking plays a key role in our understanding of nature. In a relativistic field theory, a broken continuous symmetry leads to the emergence of two types of fundamental excitations: massless Nambu-Goldstone modes and a massive 'Higgs' amplitude mode. An excitation of Higgs type is of crucial importance in the standard model of elementary particles [137] and also appears as a fundamental collective mode in quantum many-body systems [20]. Whether such a mode exists in low-dimensional systems as a resonance-like feature or becomes over-damped through coupling to Nambu-Goldstone modes has been a subject of theoretical debate [20,36-38, 40-42, 138]. Here we experimentally reveal and study a Higgs mode in a two-dimensional neutral superfluid close to the transition to a Mott-insulating phase. We identify the mode by observing the expected softening of the onset of spectral response when approaching the quantum critical point. In this regime, our system is described by an effective relativistic field theory with a two-component quantum-field [20,138] which constitutes a minimal model for spontaneous breaking of a continuous symmetry. Additionally, all microscopic parameters of our system are known from first principles and the resolution of our measurement allows us to detect excited states of the many-body system at the level of individual quasiparticles. This allows for an in-depth study of Higgs excitations, which also addresses the consequences of reduced dimensionality and confinement of the system. Our work constitutes a first step in exploring emergent relativistic models with ultracold atomic gases. 


\section{Introduction to amplitude and phase modes}

\subsection{Overview}

Higgs modes are amplitude oscillations of a quantum field and appear as collective excitations in quantum many-body systems as a consequence of spontaneous breaking of a continuous symmetry. Close to a quantum critical point, the low-energy physics of such systems is in many cases captured by an effective Lorentz invariant critical theory [20]. The minimal version of such a theory describes the dynamics of a complex order parameter $\Psi=|\Psi| e^{i \phi}$ near a quantum phase transition between an ordered $(|\Psi|>0)$ and a disordered phase $(|\Psi|=0)$. Within the ordered phase, the classical energy density has the shape of a Mexican hat (Fig. 8.1) and the order parameter takes on a non-zero value in the minimum of this potential. Hereby, its phase $\phi$ acquires a definite value through spontaneous breaking of the rotation symmetry (i.e., $U(1)$ symmetry). Expanding the field around the symmetry broken ground state leads to two types of modes: a Nambu-Goldstone mode and a Higgs mode related to phase and amplitude variations of $\Psi$, respectively (Fig. 8.1). In contrast to the phase mode, the amplitude mode has a finite excitation gap (i.e., a finite mass), which is expected to show a characteristic softening when approaching the disordered phase (Fig. 8.1). The sketched minimal model of an order parameter with $N=2$ components belongs to a class of $O(N)$ relativistic field theories, which are essential for the study of quantum phase transitions [20].

Despite the fundamental nature of the amplitude mode, a full theoretical understanding of it has not yet been achieved. In particular, the decay of the amplitude mode into lower lying phase modes, especially in two dimensions, has led to a considerable theoretical interest concerning the observability of the mode: does a resonance-like feature of the amplitude mode persist, or does the decay result in a low-frequency divergence [20, 36-41]?

The earliest experimental evidence for a Higgs mode stems from Raman scattering in a superconducting charge-density wave compound showing an unexpected peak [139], which was later interpreted as a signal of an amplitude mode [140]. Further examples of experiments in solid-state systems can be found in Ref. [40]. Importantly, none of these experiments have studied the mode spectrum across a quantum phase transition, except for neutron scattering experiments on quantum antiferromagnets [141]. In contrast to the work presented here, a resonance-like response of an amplitude mode is undoubtedly expected in these systems, because the phase transition occurs in three dimensions. 


\section{Introduction to amplitude and phase modes}

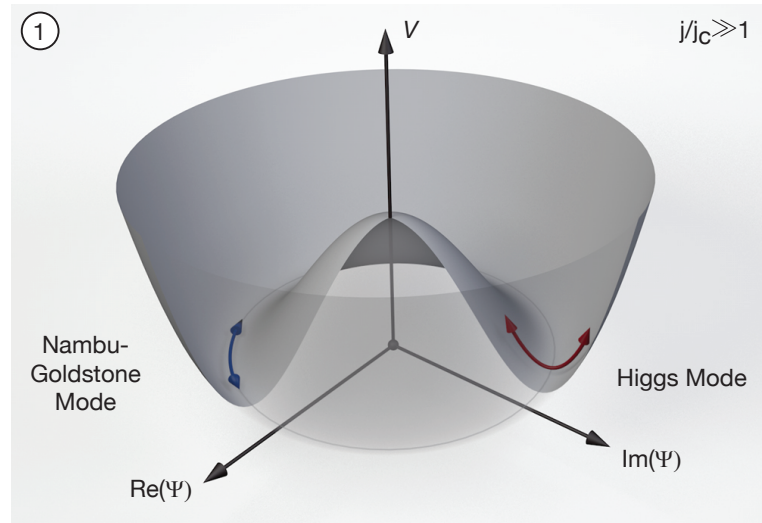

(2)

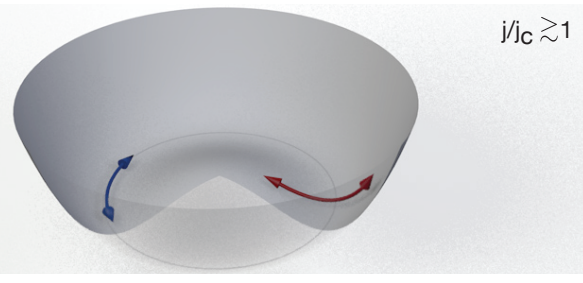

(3)

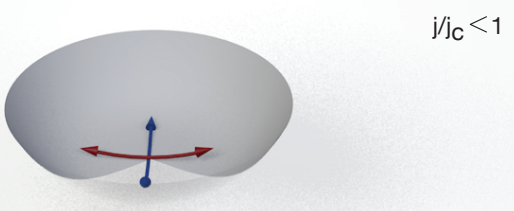

Figure 8.1.: Illustration of the Higgs mode a, Classical energy density $V$ as a function of the order parameter $\Psi$. Within the ordered (superfluid) phase, Nambu-Goldstone and Higgs modes arise from phase and amplitude modulations (blue and red arrows in panel 1). With the coupling $j=J / U$ (see main text) approaching the critical value $j_{c}$, the energy density transforms into a function with a minimum at $\Psi=0$ (panels 2-3). Simultaneously, the curvature in the radial direction softens, leading to a characteristic reduction of the excitation frequency for the Higgs mode. In the disordered (Mott-insulating) phase, two gapped modes exist, corresponding to particle and hole excitations in our case (red and blue arrow in panel 3). 
Ultracold bosonic atoms in optical lattices offer unique possibilities to study quantum phase transitions in a reduced dimensionality [3]. As shown in Sec. 2.4, these systems are nearly ideal realizations of the Bose-Hubbard model, which is parametrized by a tunnelling amplitude $J$ and an on-site interaction energy $U$. The coupling parameter $j=J / U$ is easily tunable via the lattice depth and the dimensionality of the system can be reduced by suppressing hopping in a certain direction (Sec.5). At a critical coupling $j_{c}$ and commensurate filling, the system undergoes a quantum phase transition from a superfluid (ordered) to a Mott-insulating (disordered) phase [3], which is described by an $O(2)$ relativistic field theory [20,138, 142].

A number of theoretical works have studied the Higgs mode in this system [41, 49, 52, 138, 143-146]. In particular, it has been argued that a modulation of the lattice depth can reveal a Higgs mode even in a two-dimensional system [40, 41]. Previous experiments using a lattice modulation amplitude of $20 \%$ were unable to identify the gapped amplitude mode [32, 33], most likely owing to the strong nonlinear drive [34]. A recent theoretical analysis of experiments using Bragg scattering in three-dimensional superfluids interpreted parts of the measured spectrum to be the result of non-linear coupling to a short-wavelength amplitude mode [147]. Here we experimentally study the long-wavelength and low-energy response, which is described by a relativistic field theory at the quantum critical point. 


\subsection{Amplitude and phase modes in the $O(2)$ model}

The low-energy dynamics of the Bose-Hubbard model close to the superfluid-Mottinsulator transition is described by an effective field theory with Lagrangian density $[18,20,138,142]$

$$
\begin{aligned}
\mathcal{L} & \propto\left|\left(\partial_{t}+i \delta \mu\right) \Psi\right|^{2}-c_{s}^{2}|\nabla \Psi|^{2}-V(\Psi), \\
V(\Psi) & =r|\Psi|^{2}+u|\Psi|^{4},
\end{aligned}
$$

where $c_{S}$ is the sound velocity and $\Psi$ is a space and time-dependent complex function. We defined $\delta \mu=\mu-\bar{\mu}$ as the difference of the actual chemical potential $\mu$ to the chemical potential $\bar{\mu}$ that would yield an integer average occupation number $\bar{n}$ (Fig. 8.2).

For $\bar{n} \gg 1$, the sound velocity $c_{S}$ and the pre-factors $r$ and $u$ in the 'potential' energy density $V$ can be approximately parametrized in terms of the original Bose-Hubbard parameters as $[138,142]$

$$
\begin{aligned}
c_{s} & \approx 2 J \bar{n} \sqrt{z} \\
r & \approx(2 J \bar{n} z)^{2}\left(\frac{U}{4 J \bar{n} z}-1\right) \\
u & \approx \frac{J U z}{4},
\end{aligned}
$$

where $z$ is the number of nearest neighbors. We expect the dependence for low filling $\bar{n}$ to be qualitatively similar to this result. Here we measure lengths in units of the lattice spacing $a_{\text {lat }}$ and we set $\hbar=1$.

The effective field-theory description is considered to be valid only close to the superfluid-Mott-insulator phase-transition boundary. Despite this limitation it is an important guide for the qualitative and, to some degree, quantitative understanding of the mode spectrum. In the following, we first discuss the $\delta \mu=0$ case, i.e. the tip of the lobe transition (Fig. 8.2). We will come back to the influence of a finite $\delta \mu$ in Sec. 8.5.

Depending on the sign of $r$, the potential $V(\Psi)$ takes on the shape of a Mexican hat $(r<0)$ or a shape with only a single minimum in the center $(r>0$, see Fig. 8.1). Within a mean-field approximation, the transition point is at $r=0$, and the equilibrium value $\Psi_{0}$ is found by minimizing $V\left(\Psi_{0}\right)$. This yields $\left|\Psi_{0}\right|=0$ for $r>0$ in the Mott-insulating phase and

$$
\left|\Psi_{0}\right|=\sqrt{-\frac{r}{2 u}}>0
$$

for $r<0$ in the superfluid phase.

Due to the rotational symmetry of $V(\Psi)$, only the absolute value of $\left|\Psi_{0}\right|$ takes on a 


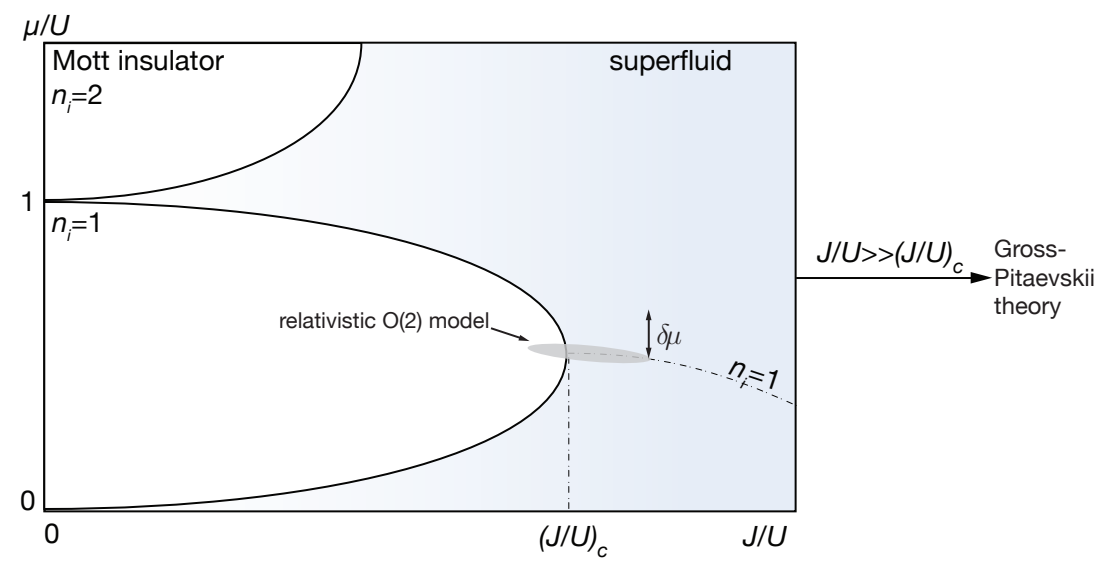

Figure 8.2.: Effective low-energy descriptions. At integer filling, close to the tip of the Mottinsulating lobe, we expect a description in terms of an effectively relativistic field theory. In the weakly interacting limit, the dynamics is described by Gross-Pitaevskii theory. Both descriptions lead to a different excitation spectrum (Sec. 8.4). The relativistic description allows for an independent gapped amplitude Higgs mode, while in Gross-Pitaevskii theory, only a gapless Bogoliubov mode exists.

specific value. The phase of $\Psi_{0}$ takes on a definite value by spontaneous breaking of this global $U(1)$ symmetry.

\subsubsection{Relation of $\Psi$ to observables}

Before dealing with the dynamics of $\Psi$, we would like to connect $\Psi$ to actual observables of the system. For this, we follow the notation and derivation of Ref. [138, 142], which is based on a mean-field description with mean-field states

$$
\begin{aligned}
|\Omega\rangle_{M F} & =\prod_{j}\left|\psi_{j}\right\rangle_{M F} \\
\left|\psi_{j}\right\rangle_{M F} & \approx \cos \left(\theta_{j} / 2\right)|\bar{n}\rangle_{j}+\frac{1}{\sqrt{2}} \sin \left(\theta_{j} / 2\right)\left(e^{i \phi_{j}}|\bar{n}+1\rangle_{j}+e^{\left.-i \phi_{j}|\bar{n}-1\rangle_{j}\right),}\right.
\end{aligned}
$$

where $\theta_{j}$ and $\phi_{j}$ are variational parameters. Apart from the mean-field assumption that the state of the system is described by a product of on-site states, an additional assumption is made that only states with $\bar{n}_{j}$ and $\bar{n}_{j} \pm 1$ contribute significantly. This is reasonable close to the two-dimensional transition as can be seen by the experimentally observed suppression of number fluctuations (see Sec. 5.4).

The approximate ground state of the system is found by minimizing the energy functional $\left\langle\left.\Omega\right|_{M F} \hat{H}_{B H} \mid \Omega\right\rangle_{M F}$. In Eq. 8.8, we made the additional approximation that the states with $|\bar{n}+1\rangle_{j}$ and $|\bar{n}-1\rangle_{j}$ appear with the same quantum-mechanical probability. This assumption yields the minimal variational energy in the limit $\bar{n} \gg 1$, but is 


\section{Introduction to amplitude and phase modes}

still a good approximation for $\bar{n}=1$ [138] (see Sec. 9.9). Within this scheme, the Mottinsulating phase is reached for $\theta_{j}=0$ and, consequently, number fluctuations are completely suppressed. Number fluctuations only appear in the superfluid phase, for which we find $\theta_{j}>0$. In all cases, the energy functional is independent of the phase $\phi_{j}$.

The relation to the order parameter $\Psi_{j}$ at site $j$ is given by

$$
\Psi_{j}=\frac{1}{2} \sin \left(\theta_{j}\right) e^{i \phi_{j}} \propto \frac{1}{\sqrt{n}}\left\langle\left.\Omega\right|_{M F} \hat{b}_{j} \mid \Omega\right\rangle_{M F}
$$

The order parameter is therefore proportional to the expectation value of the destruction operator $\hat{b}_{j}$ in the mean-field state. The amplitude of $\Psi_{j}$, which is proportional to $\sin \left(\theta_{j}\right)$, is related to the variance of the on-site occupation number $\sigma_{j}^{2}$, which is given by $\sigma_{j}^{2}=\left\langle\left(\hat{n}_{j}-\bar{n}\right)^{2}\right\rangle=\sin \left(\theta_{j} / 2\right)^{2}$.

However, the amplitude of the order parameter is maximal for $\theta_{j}=\frac{\pi}{2}$, which yields $\sin \left(\theta_{j} / 2\right)=\cos \left(\theta_{j} / 2\right)=1 / \sqrt{2}$. In this case, we have a probability of $1 / 2$ to find $\bar{n}_{j}$ and a probability of $1 / 2$ to find one of the 'excited' states with $\bar{n}_{j} \pm 1$. In contrast, maximum number fluctuations are found for $\theta_{j}=\pi$. In this case, the probability to find $\bar{n}_{j}$ is zero. A finite amplitude of $\Psi_{j}$, therefore, indicates a coherent superposition of all three states with $\bar{n}_{j}$ and $\bar{n}_{j} \pm 1$. The amplitude of $\Psi_{j}$ vanishes if the system is either with certainty in a state with $\bar{n}_{j}$ or with certainty in one of the states with $\bar{n}_{j} \pm 1$.

The phase of the order parameter $\phi_{j}$ is the relative phase between the states with an extra particle and a missing particle. A finite phase difference $\phi_{k}-\phi_{k+1}$ between neighboring sites leads to a probability current flowing between these sites. The lattice probability current is given by the current operator $\hat{j}_{k}=-i J\left(\hat{b}_{k+1}^{+} \hat{b}_{k}-\hat{b}_{k}^{+} \hat{b}_{k+1}\right)$, which appears in the continuity equation $\frac{d \hat{n}_{k}}{d t}=\hat{j}_{k}-\hat{j}_{k-1}$ (see, e.g., Ref. [148]). The resulting current $\left\langle\left.\Omega\right|_{M F} \hat{j}_{k} \mid \Omega\right\rangle_{M F}$ is then proportional to $\sin \left(\phi_{k}-\phi_{k+1}\right)$.

\subsubsection{Relativistic form and free Lagrangian}

After getting a better intuition for the meaning of $\Psi$, we deal with the dynamics described by the Lagrangian in Eq. 8.1 for $\delta \mu=0$. One of the key features is that the Lagrangian can be written in an effectively relativistic form in this case. We define the four-derivatives $\partial_{v}=\left(\frac{1}{c_{s}} \partial_{t}, \nabla\right)$ and $\partial^{v}=\left(\frac{1}{c_{s}} \partial_{t},-\nabla\right)$ and rewrite $\mathcal{L}$ as

$$
\mathcal{L} \propto c_{S}^{2} \partial_{\nu} \Psi^{*} \partial^{\nu} \Psi-r|\Psi|^{2}-u|\Psi|^{4},
$$

using the Einstein summation convention.

In this form, the Lagrangian is invariant under a Lorentz transformation, where the 
speed of light $c$ is substituted by the sound velocity $c_{S}$.

Up to an additive constant, we can write $\mathcal{L}$ as

$$
\mathcal{L} \propto c_{S}^{2} \partial_{\nu} \Psi^{*} \partial^{\nu} \Psi-u\left(|\Psi|^{2}-\left|\Psi_{0}\right|^{2}\right)^{2},
$$

where $\left|\Psi_{0}\right|$ is defined in Eq. 8.6. The model in this form is usually referred to as the relativistic $O(2)$ model, i.e., a model with rotation invariance of a two-component order parameter.

Our interest is in small deviations $\phi$ from the equilibrium value $\Psi_{0}$, defined as

$$
\begin{aligned}
& \phi=\Psi-\Psi_{0} \\
& \phi=\phi_{1}+i \phi_{2} .
\end{aligned}
$$

We now 'break the global $U(1)$ symmetry' and pick an equilibrium solution $\Psi_{0}$ that is real. Then the real part of the deviation, $\phi_{1}$, corresponds to variations in the amplitude direction of $\Psi_{0}$, whereas a small $\phi_{2}$ represents a variation in phase direction. We can now expand this Lagrangian in $\phi_{1}$ and $\phi_{2}$, which yields

$$
\begin{aligned}
\mathcal{L} & \propto \mathcal{L}_{\text {free }}+\mathcal{L}_{\text {int }} \\
\mathcal{L}_{\text {free }} & =c_{s}^{2} \partial_{v} \phi_{1} \partial^{v} \phi_{1}-4 u \Psi_{0}^{2} \phi_{1}^{2}+c_{s}^{2} \partial_{v} \phi_{2} \partial^{v} \phi_{2},
\end{aligned}
$$

where $\mathcal{L}_{\text {int }}$ contains higher order terms in $\phi_{1}, \phi_{2}$. For now, we stick to the free evolution described by $\mathcal{L}_{\text {free }}$ and treat the interaction part $\mathcal{L}_{\text {int }}$ later (Sec. 8.6).

\subsubsection{Amplitude and phase modes}

We can now interpret the deviations $\phi_{1}$ and $\phi_{2}$ as new fundamental variables. The corresponding equations of motion are the Euler-Lagrange equations $\frac{\partial \mathcal{L}}{\partial \phi_{i}}-\partial_{\nu} \frac{\partial \mathcal{L}}{\partial \partial_{\nu} \phi_{i}}=$ 0 for $i=1,2$. For the moment, we are only interested in the free evolution that is independent of $\mathcal{L}_{\text {int }}$. Setting up the Euler-Lagrange equations with $\mathcal{L}_{\text {free }}$ instead of $\mathcal{L}$ yields

$$
\begin{aligned}
& \ddot{\phi}_{1}=c_{s}^{2} \nabla^{2} \phi_{1}-\Delta_{0}^{2} \phi_{1} \\
& \ddot{\phi}_{2}=c_{s}^{2} \nabla^{2} \phi_{2},
\end{aligned}
$$

where we additionally defined the energy-gap $\Delta_{0}$ as

$$
\Delta_{0}^{2}=4 u \Psi_{0}^{2}=-2 r .
$$

Eq. 8.16 for $\phi_{1}$ is formally equivalent to a Klein-Gordon equation for a particle with mass $m$ if we substituted $\Delta_{0}=m c_{s}^{2}$. Both equations of motion are solved by real superpositions of plane waves

$$
\begin{aligned}
& \phi_{1} \propto e^{i\left(\omega_{1} t-k_{1} x\right)} \\
& \phi_{2} \propto e^{i\left(\omega_{2} t-k x\right)},
\end{aligned}
$$




\section{Introduction to amplitude and phase modes}

where the dispersion relations (Fig. 8.3) are given by

$$
\begin{aligned}
& \omega_{1}^{2}=\Delta_{0}^{2}+c_{s}^{2} k_{1}^{2} \\
& \omega_{2}^{2}=c_{s}^{2} k_{2}^{2} .
\end{aligned}
$$

The dispersion for $\omega_{1}$ is formally equivalent to a relativistic energy-momentum relation. The gap $\Delta_{0}$ plays the role of the rest-energy $m c_{s}^{2}$. A gapped mode of this kind is therefore sometimes called massive.

The interpretation of this results is as follows. On top of a homogeneous equilibrium state $\Psi_{0}$, which we chose to be real, the system may be excited by small fluctuations in the amplitude direction with $\Psi_{0}+\phi(x, t)_{1}$ or in the phase direction with $\Psi_{0}+i \phi(x, t)_{2}$, where $\phi(x, t)_{1}, \phi(x, t)_{2}$ are chosen to be real (Fig. 8.3). The excitations in an infinite homogeneous system take the form of plane waves that propagate in a fully decoupled fashion. The lowest energy fluctuations are given by waves with small momenta $k_{1}$ and $k_{2}$ that correspond to long wavelengths and, therefore, to changes in the system on the longest length scale. Such smooth variations in phase direction $\phi_{2}$ can be created with an arbitrarily small energy because $\omega_{2} \rightarrow 0$ for $k_{2} \rightarrow 0$, i.e. the modes are gapless Nambu-Goldstone modes [149, 150]. The dispersion relation for modes in amplitude direction $\phi_{1}$ shows a finite energy gap $\Delta_{0}$ for $k_{1} \rightarrow 0$, i.e. they are gapped modes. We refer to these modes as Higgs amplitude modes (see also Sec. 8.3).

From the discussion below Eq. 8.9, we conclude that a phase mode leads to a probability current in $k$-direction. In contrast, Higgs amplitude modes are traveling waves that describe a variation of the degree of local number-fluctuations. The $k=0$ Higgs amplitude mode leads to a global oscillation of number-fluctuations with frequency $\Delta_{0}$ at almost constant mean density (see also Ref. [49]).

\subsubsection{Mode softening}

The square of the energy gap $\Delta_{0}^{2}=-2 r$ is proportional to the curvature in amplitude direction of the potential energy $V(\Psi)$. Therefore, oscillations of $\phi_{1}$ can indeed by visualized by oscillations in the Mexican hat-shaped potential in amplitude direction (Fig. 8.1). As we approach the Mott-insulating phase the potential gradually turns into a potential with a single minimum. At the same time, the curvature is reduced and vanishes at the critical point. As a consequence, the mode gap $\Delta_{0}$ shows a characteristic reduction approaching the critical point. Since $\Delta_{0}$ sets the minimal oscillation frequency for a Higgs amplitude mode, we speak of mode softening. 

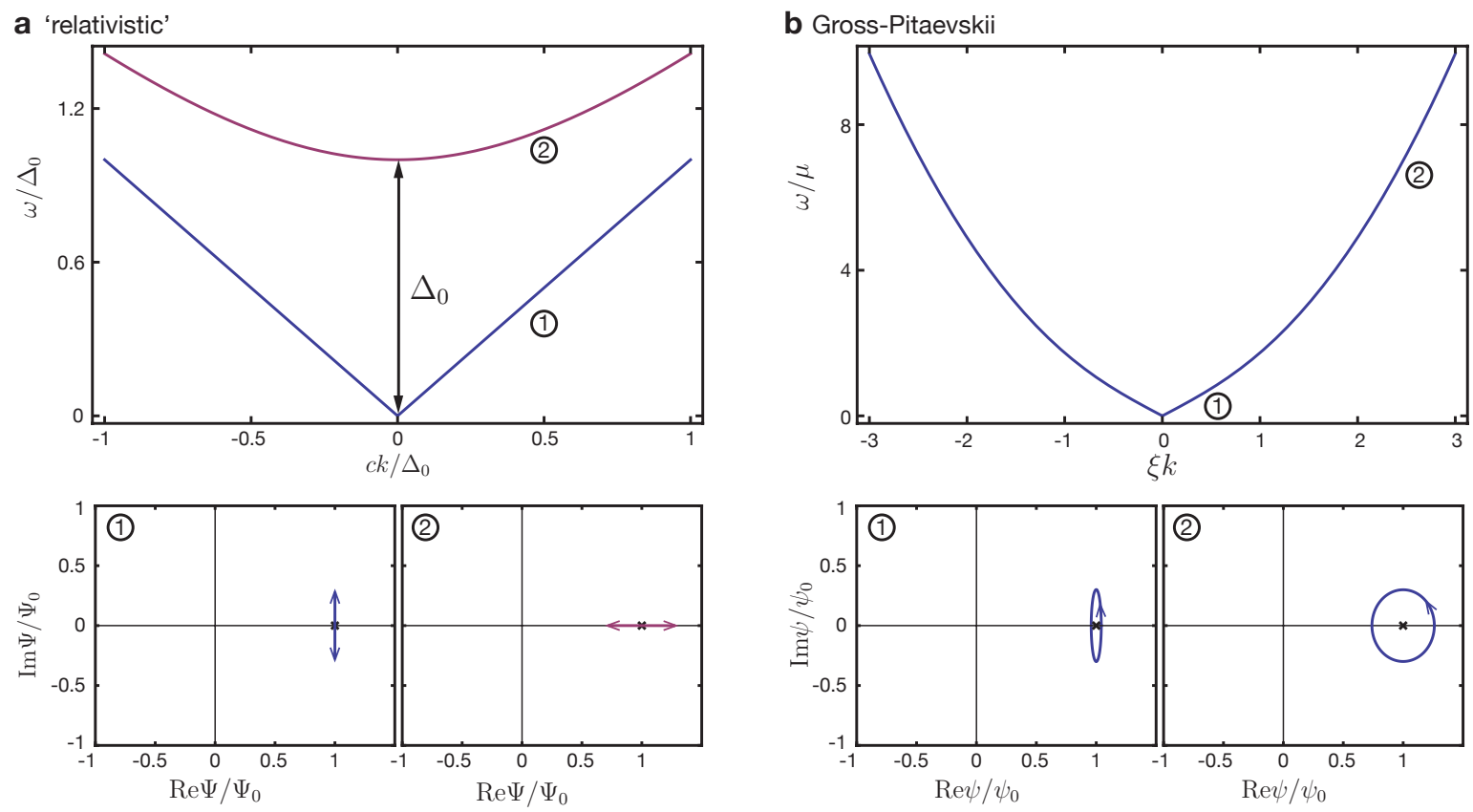

Figure 8.3.: Comparison of 'relativistic' and Gross-Pitaevskii dynamics. a, Top panel: 'relativistic' excitation spectrum based on Eq. 8.21 with the amplitude branch in purple and the phase mode branch in blue. Bottom panels: Trajectory of $\Psi(t)$ in the complex plane for an amplitude (left) and a phase mode (right). b, Top panel: Bogoliubov excitation spectrum, based on Eq. 8.38. Bottom panels: Trajectory of $\Psi(t)$ in the complex plane for a mode with small momentum $\xi k=0.5$ (left) and with large momentum $\xi k=2.5$ (right) based on Eq. 8.39. The 'relativistic' spectrum in a shows an additional gapped mode with gap $\Delta_{0}$ (arrow in top panel of a). This mode corresponds to amplitude oscillations of the order parameter (right bottom panel of a). Within Gross-Pitaevskii theory, amplitude and phase oscillations are coupled and only a single mode exists. In general, these modes describe ellipses in the complex plane (bottom panels of $\mathbf{b}$ ). For low momenta, the ellipses are strongly asymmetric with their major axis along the phase direction. For large momenta, they become increasingly circular. The trajectories for the 'relativistic' theory are completely along the amplitude or phase direction, independent of the momentum $k$. 


\section{Introduction to amplitude and phase modes}

\subsection{Anderson-Higgs mechanism}

We would like to stress that the previously described model does not show the Anderson-Higgs mechanism, but exhibits an amplitude Higgs mode. To explain the difference in more detail, we will investigate an extension of the model defined in Eq. 8.10 that incorporates breaking of a gauge symmetry. The model we will use is indeed the one studied by Higgs in Ref. [151]. Our explanation follows Ref. [35].

We consider the the Lagrangian density

$$
\mathcal{L}_{A H} \propto\left[\left(\partial_{\nu}-i q A_{v}\right) \Psi^{*}\right]\left[\left(\partial^{v}+i q A v\right) \Psi\right]-\frac{1}{4} F_{\mu v} F^{\mu v}-u\left(|\Psi|^{2}-\left|\Psi_{0}\right|^{2}\right)^{2},
$$

where we set $c=1$ and $F_{\mu \nu}=\partial_{\mu} A_{\nu}-\partial_{\nu} A_{\mu}$ is the field-strength tensor in terms of the electromagnetic vector potential $A_{v}$. The coupling constant $q$ quantifies the coupling between the 'Higgs' field $\Psi$ and the gauge field $A_{v}$.

Assume for a moment that there was no coupling between $\Psi$ and $A_{v}(q=0)$. In this case the Lagrangian $\frac{1}{4} F_{\mu v} F^{\mu v}$ would lead to (massless) electromagnetic waves in two polarization states. The part of $\mathcal{L}_{A H}$ involving $\Psi$ would lead to a massive Higgs amplitude mode and to a massless Nambu-Goldstone mode, as shown in the previous section.

Including a finite coupling $q \neq 0$, the Lagrangian $\mathcal{L}_{A H}$ is invariant under a (local) $U(1)$ gauge transformation

$$
\begin{aligned}
\Psi(x) & \rightarrow \Psi(x) e^{-i q \Phi(x)} \\
A_{v}(x) & \rightarrow A_{v}(x)+\partial_{\nu} \Phi(x) .
\end{aligned}
$$

Note that this involves a varying phase $\Phi(x)$, whereas the model in Eq. 8.11 is only invariant under a global transformation $\Psi \rightarrow \Psi e^{i \Phi}$.

One possibility to fix a gauge, and hence to break the local $U(1)$ gauge symmetry, is to pick a real $\Psi$ at any point in space and time. For a given complex $\Psi(x)=|\Psi(x)| e^{i \Phi(x)}$, this can always be obtained by a transformation $\Psi(x) \rightarrow \Psi(x) e^{-i q \tilde{\Phi}(x) / q}$.

One can now expand $\mathcal{L}_{A H}$ using $\Psi(x)=\Psi_{0}+h(x)$, where $\Psi_{0}$ and $h(x)$ are real, yielding

$$
\begin{aligned}
\mathcal{L}_{A H} & \propto \mathcal{L}_{A H, \text { free }}+\mathcal{L}_{A H, \text { int }} \\
\mathcal{L}_{A H, \text { free }} & \propto \partial_{\nu} h \partial^{v} h-4 u \Psi_{0}^{2} h^{2}-\frac{1}{4} F_{\mu \nu} F^{\mu v}+q^{2} \Psi_{0}^{2} A_{v} A^{v},
\end{aligned}
$$

where $\mathcal{L}_{A H \text {,int }}$ includes higher order terms. The free Lagrangian $\mathcal{L}_{A H \text {,free }}$ splits into two parts that describe the independent dynamics of $h$ and $A_{v}$.

The first part, involving $h$, has the same form as the Lagrangian for $\phi_{1}$ in the previous section (Eq. 8.15) and is independent of $q$. It yields a massive mode related to the 
amplitude of $\Psi$ with a mass proportional to $\left(u \Psi_{0}^{2}\right)^{1 / 2}$. The coupling to the electromagnetic field has not changed the free part of the Lagrangian for the amplitude degree of freedom. In this sense, there is no fundamental difference between an amplitude Higgs mode in an $O(2)$ system with global symmetry and in a system with (local) gauge symmetry. Only the interaction of the amplitude mode with the remaining degrees of freedom, described by $\mathcal{L}_{A H \text {,int }}$, is modified.

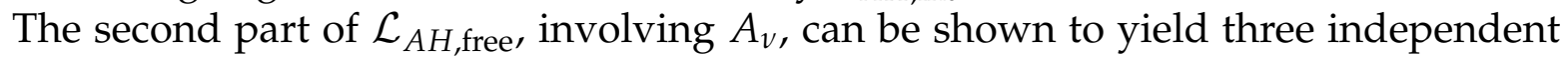
modes [35]. The crucial point is that due to the gauge fixing procedure, a mass term $q^{2} \Psi_{0}^{2} A_{v} A^{v}$ appears, i.e. the modes of the vector gauge field acquired a mass proportional to $q \Psi_{0}$. In general, the process of mass generation for a gauge field due to breaking of a gauge symmetry is known as the Anderson-Higgs mechanism.

Electro-weak symmetry breaking in the Standard model is based on a generalization of this concept incorporating a $U(1) \times \mathrm{SU}(2)$ gauge symmetry and associated vector gauge fields. The Higgs field $\Psi$, in this case, has two complex components instead of only one. After breaking of the $\mathrm{SU}(2)$ symmetry, the resulting Lagrangian shows massive vector gauge fields, corresponding to the $W^{+}, W^{-}$and $Z$ particles that mediate the weak interaction, and a massless vector gauge field, corresponding to photons that mediate the electromagnetic interaction. Similar to the previous examples, there is also a massive mode related to amplitude oscillations of the Higgs field $\Psi$, which is commonly known as the Higgs particle.

We stress that the free part of the Lagrangian describing the Higgs particle in this theory has exactly the same form as in the previously described theories that had only a global or local $U(1)$ invariance. There is no mathematical difference on the level of free Lagrangians concerning the Higgs mode. However, the coupling to other modes and therefore the decay of the Higgs particle is different. Also there is no simple gapless phase mode in the case of local symmetry breaking.

\subsection{Comparison with Gross-Pitaevskii dynamics}

It is instructive to compare the dynamics described by Eq. 8.10 with the low-energy dynamics of a weakly-interacting Bose gas, described by a Gross-Pitaevskii equation (see, e.g., Ref. [71]).

\section{'Relativistic' dynamics}

For the comparison, it is useful to derive the equations of motion, including interaction terms, directly from Eq. 8.10 without splitting into real and imaginary fluctuations. The equation of motion for the field $\Psi$ is given by the Euler-Lagrange equation 


\section{Introduction to amplitude and phase modes}

$\frac{\partial \mathcal{L}}{\partial \Psi^{*}}-\partial_{\nu} \frac{\partial \mathcal{L}}{\partial \partial_{\nu} \Psi^{*}}=0$ for the complex conjugate field $\Psi^{*}$, which yields [138]

$$
\ddot{\Psi}=c^{2} \nabla^{2} \Psi-\frac{1}{2} \Delta_{0}^{2} \Psi\left(\frac{|\Psi|^{2}}{\left|\Psi_{0}\right|^{2}}-1\right),
$$

where $\left|\Psi_{0}\right|^{2}=-r / 2 u$ is the equilibrium value of $\Psi$. This is a non-linear equation where, in particular, the real and complex part of $\Psi$ are coupled. One can again pick a real $\Psi_{0}$ and expand in small deviations from the mean value (Eq.8.12 and 8.13). The resulting equations for the deviations $\phi_{1}$ in amplitude and the deviations $\phi_{2}$ in phase direction are the same as Eq.8.16 and 8.17. As solutions, we found two independent modes: one gapped mode in amplitude direction and a gappless mode in phase direction.

\section{Gross-Pitaevskii dynamics}

The time-dependent Gross-Pitaevksii equation for the condensate wavefunction $\psi$ in a homogeneous system is

$$
-i \hbar \dot{\psi}=\frac{\hbar^{2}}{2 m} \nabla^{2} \psi-g|\psi|^{2} \psi,
$$

where $g=4 \pi \hbar^{2} a_{s} / m$ is the interaction strength, $a_{s}$ is the s-wave scattering length (see also Eq. 2.18) and $m$ is the atomic mass. In the following discussion, we set $\hbar=1$. We employ a transformation to a rotating frame $\psi \rightarrow \psi e^{-i \mu t}$, which yields

$$
-i \dot{\psi}=\frac{1}{2 m} \nabla^{2} \psi-g \psi\left(|\psi|^{2}-\frac{\mu}{g}\right) .
$$

The equilibrium solution is

$$
\left|\psi_{0}\right|^{2}=\frac{\mu}{g}=n
$$

where $n$ is the condensate-density. With this, we can write the Gross-Pitaevskii equation as

$$
-i \dot{\psi}=\frac{1}{2 m} \nabla^{2} \psi-\mu \psi\left(\frac{|\psi|^{2}}{\left|\psi_{0}\right|^{2}}-1\right) .
$$

This has the same structural form as the 'relativistic' Eq. 8.27, except that there is a first order time-derivative on the left-hand side instead of a second-order one. We will show now that, as a consequence, there is only a single excitation branch. Again, 
our interest is in small fluctuations around the equilibrium value $\psi_{0}$. Let us pick a real $\psi_{0}$ and write the fluctuations as

$$
\begin{gathered}
\delta=\psi-\psi_{0} \\
\delta=\delta_{1}+i \delta_{2}
\end{gathered}
$$

Linearizing Eq. 8.31 in $\delta$ yields

$$
-i \dot{\delta}=\frac{1}{2 m} \nabla^{2} \delta-\mu\left(\delta+\delta^{*}\right),
$$

which is equivalent to

$$
\begin{aligned}
-\dot{\delta}_{1} & =\frac{\hbar^{2}}{2 m} \nabla^{2} \delta_{2} \\
\dot{\delta}_{2} & =\frac{\hbar^{2}}{2 m} \nabla^{2} \delta_{1}-2 \mu \delta_{1} .
\end{aligned}
$$

Already in linear order, amplitude and phase fluctuations are coupled, which is a direct consequence of the term $-i \dot{\delta}$ on the left-hand side of Eq. 8.31. These equations can be solved with a Bogoliubov transformation

$$
\delta=u e^{i(\omega t-k x)}+v^{*} e^{-(i \omega t-k x)} .
$$

The dispersion relation is given by

$$
(\hbar \omega)^{2}=\mu^{2} \tilde{k}^{2}\left(\tilde{k}^{2}+2\right)
$$

where we introduced the normalized momentum $\tilde{k}=\xi k$, with the healing length $\xi$ given by $\tilde{\xi}^{2}=\frac{\hbar^{2}}{2 m \mu}$. There is only a single excitation branch with $\omega \rightarrow 0$ for $k \rightarrow 0$, i.e. we find a gapless spectrum (Fig. 8.3b).

These Bogoliubov modes are coupled phase and amplitude modes as can be seen by investigating the trajectory of $\delta(t)$ as a function of time $t$ in the complex plane. For simplicity, we choose $x=0$ and $\delta(0)$ along the real axis (i.e., $u$ and $v$ are real). With this, one finds

$$
\begin{aligned}
& \delta_{1}=u\left(1+\frac{v}{u}\right) \cos (\omega t)=u\left(\tilde{k} \sqrt{\tilde{k}^{2}+2}-\tilde{k}^{2}\right) \cos (\omega t) \\
& \delta_{2}=u\left(1-\frac{v}{u}\right) \sin (\omega t)=u\left(2-\tilde{k} \sqrt{\tilde{k}^{2}+2}+\tilde{k}^{2}\right) \sin (\omega t) .
\end{aligned}
$$

In general, these equations describe elliptic trajectories. For small momenta $k$, the ellipse has its major axis along the imaginary axis and the modes are predominantly in the phase direction (Fig. 8.3b). In the limit $k \rightarrow 0$, we find a pure phase mode. In contrast, for large momenta we find almost circular trajectories. This general 


\section{Introduction to amplitude and phase modes}

behavior is independent of the position $x$ and the initial condition for $\delta(0)$.

In summary, we found only a single gapless excitation branch, which is phase-like for small momenta. There is no independent amplitude mode in the Gross-Pitaevskii description. This is the case despite the fact that one can derive the Gross-Pitaevskii Equation in Eq. 8.31 from a Lagrangian that has a Mexican hat-shaped potential and that the theory posses a global $U(1)$ symmetry. These properties are not sufficient for the existence of a Higgs amplitude mode. The crucial difference is, indeed, the appearance of a second order time derivative in the equations of motion.

\subsection{Influence of deviation from integer filling}

For a density that deviates from integer filling, we have to include $\delta \mu \neq 0$. The Lagrangian density in Eq. 8.1 can be written as

$$
\begin{aligned}
\mathcal{L} & \propto|\dot{\Psi}|^{2}+i \delta \mu \Psi \dot{\Psi}^{*}-i \delta \mu \Psi^{*} \dot{\Psi}-c^{2}|\nabla \Psi|^{2}-V(\Psi) \\
V(\Psi) & =\left(r-\delta \mu^{2}\right)|\Psi|^{2}+u|\Psi|^{4} .
\end{aligned}
$$

Notice that the mean-field transition point acquired a shift and is now given by $r=\delta \mu^{2}$. This corresponds to a shift to lower $J / U$ values (see Eq. 8.4) and defines the shape of the Mott lobe within the present description. The mean field equilibrium order parameter reads

$$
\left|\tilde{\Psi}_{0}\right|^{2}=\frac{-r+\delta \mu^{2}}{2 u},
$$

and the Euler-Lagrange equation is given by

$$
\ddot{\Psi}+2 i \delta \mu \dot{\Psi}=c^{2} \nabla^{2} \Psi-\left(-r+\delta \mu^{2}\right) \Psi\left(\frac{|\Psi|^{2}}{\left|\tilde{\Psi}_{0}\right|^{2}}-1\right) .
$$

We observe a mixed relativistic and Gross-Pitaevskii dynamics as can be seen from the appearance of first and second order time-derivatives. Eq. 8.43 can be linearized and afterwards solved using a Bogoliubov transformation (Eq. 8.38). This calculation results in two independent excitation branches $\omega_{ \pm}$with dispersions

$$
\begin{aligned}
\omega_{ \pm}^{2} & =\frac{1}{2}\left(2 c^{2} k^{2}+\tilde{\Delta}_{0}^{2} \pm \sqrt{16 \delta \mu^{2} c^{2} k^{2}+\tilde{\Delta}_{0}^{4}}\right) \\
\tilde{\Delta}_{0}^{2} & =-2 r+6 \delta \mu^{2}=\Delta_{0}^{2}+6 \delta \mu^{2} .
\end{aligned}
$$

Importantly, we find $\omega_{+} \rightarrow \tilde{\Delta}_{0}$ and $\omega_{-} \rightarrow 0$ for $k \rightarrow 0$. We therefore identify $\omega_{+}$ as the modified amplitude mode dispersion and $\omega_{-}$as the modified phase mode 
dispersion. Additionally, one can show that the trajectories of the modes related to $\omega_{+}\left(\omega_{-}\right)$are ellipses with their major axis along the amplitude (phase) direction. Furthermore, up to quadratic order in $\delta \mu$, the dispersion relations take on the same structural form as the relativistic dispersion in Eq. 8.21, substituting $\Delta_{0} \rightarrow \tilde{\Delta}_{0}$ and $c^{2} \rightarrow c^{2}\left(1-4 \delta \mu^{2} / \Delta_{0}^{2}\right)$. In conclusion, we find a similar result as for the relativistic dynamics for small $|\delta \mu|$.

For large deviation from integer filling (large $|\delta \mu|$ ), the amplitude branch $\omega_{+}$is pushed to high frequencies as the gap value increases with $\delta \mu^{2}$. Expanding $\omega_{-}$in $k$ yields a spectrum with the same structure as the Bogoliubov spectrum in Eq. 8.38. The low-energy spectrum, in the case of large $|\delta \mu|$, is therefore dominated by Bogoliubov excitations.

One of the important features is that the gap $\tilde{\Delta}_{o}$ does not close at the transition point if $\delta \mu \neq 0$. Instead we find $\tilde{\Delta}_{o}=2|\delta \mu|$ right at the transition. The amplitude mode, therefore, always stays at a finite frequency. This means that for large $|\delta \mu|$ the low frequency spectrum is dominated by Bogoliubov excitations even in the close vicinity of the transition. This is another way of seeing that the low-energy dynamics of the superfluid-Mott-insulator transition away from integer filling is similar to the one of a weakly interacting Bose gas, consistent with the picture of condensation of particle or hole excitations (Sec. 2.2).

The gap $\tilde{\Delta}_{o}=2|\delta \mu|$ at the transition point is the width of the Mott-insulating lobe. This amounts to the energy gap for a particle- or hole-excitation of the Mott phase depending on the transition occurring at $\delta \mu<0$ or $\delta \mu>0$, respectively. This is consistent with the picture that the amplitude mode at non-integer filling evolves into one of the excitations of the Mott insulator at the transition point $[52,146]$.

The fact that the gap $\tilde{\Delta}_{o}$ increases with $\delta \mu$ also has important consequences in the detection of the amplitude mode in a trapped system. To get a simple picture let us for now assume a local density approximation and that the central chemical potential $\mu_{0}$ is slightly larger than $\bar{\mu}$. For a given distance from the center of the trap, the local chemical potential will match $\bar{\mu}$ and we find integer filling locally. At this distance, the gap for the amplitude mode is minimal. As a consequence, any response from amplitude modes of the trapped system will occur at frequencies that are larger or equal to the gap at unity filling $\Delta_{0}$. It is, therefore, possible to detect the generic gap $\Delta_{0}$ even in a trapped system (see also Ref. [49] for a discussion). We will see in Sec. 9.4 that this is still true going beyond the local density approximation. 


\subsection{Response functions and interactions between modes}

In this section, we present basic linear response theory within the Bose-Hubbard model and within the $O(2)$ model. Finally, we discuss the influence of the interactions between Higgs and phase modes on the shape of response functions. In particular, we give an introduction to the discussion about the visibility of the Higgs mode in a two-dimensional system.

\subsubsection{Linear response theory for the Bose-Hubbard model}

Experimentally, we used a modulation of the lattice depth to detect the response of Higgs amplitude modes. To leading order, a small variation of the lattice depth $V_{0}$ leads to a variation of the tunneling amplitude $J$, while the on-site interaction $U$ stays almost constant (see Sec. 2.4). The time-dependent Hamiltonian for a periodically modulated optical lattice depth can therefore be written as

$$
\begin{gathered}
\hat{H}(t) \approx \hat{H}_{B H}-\delta(t) \hat{K} \\
\hat{K}=-J \sum_{\langle i, j\rangle} \hat{b}_{j}^{+} \hat{b}_{i} \\
\delta(t)=\delta_{0} \cos (\omega t),
\end{gathered}
$$

where $\delta_{0}$ quantifies the modulation strength and $\omega=2 \pi v$ with $v$ the modulation frequency. Thus, we find a modulation of the kinetic energy $\hat{K}$. We are interested in the absorbed total energy. The energy absorption rate is given by

$$
\frac{d E}{d t}=\frac{d\langle\hat{H}(t)\rangle_{t}}{d t}=\left\langle\frac{d \hat{H}(t)}{d t}\right\rangle_{t}=-\dot{\delta}(t)\langle\hat{K}\rangle_{t}=\delta_{0} \omega \sin (\omega t)\langle\hat{K}\rangle_{t} .
$$

Here we show an explicit time-dependence of the quantum mechanical average $\langle\ldots\rangle_{t}$ to indicate that the quantum state itself is time-dependent. The second equality can be shown using the Schrödinger equation.

Within linear response theory, the time-dependence of $\langle\hat{K}\rangle_{t}$ is (see, e.g., Refs. [20, 152])

$$
\begin{aligned}
\langle\hat{K}\rangle_{t}-\langle\hat{K}\rangle_{0} & =\int_{-\infty}^{t} d t^{\prime} \chi\left(t-t^{\prime}\right) \delta\left(t^{\prime}\right) \\
& =\delta_{0} \operatorname{Re}[\chi(\omega)] \cos (\omega t)+\delta_{0} \operatorname{Im}[\chi(\omega)] \sin (\omega t)
\end{aligned}
$$

with $\chi\left(t-t^{\prime}\right)$ the dynamical susceptibility, $\langle\hat{K}\rangle_{0}$ the equilibrium average previous to the modulation, and $\operatorname{Re}[. .$.$] and \operatorname{Im}[. .$.$] denote the real and imaginary parts respec-$ tively. We will come back to the precise form of $\chi\left(t-t^{\prime}\right)$ later and first investigate the energy absorption rate. Eq. 8.51 holds for a periodic cosine modulation, where $\chi(\omega)$ is the Fourier transform of $\chi\left(t-t^{\prime}\right)$ defined as

$$
\chi(\omega)=\int_{-\infty}^{t} d t^{\prime} \chi\left(t-t^{\prime}\right) e^{i(\omega+i \epsilon)\left(t-t^{\prime}\right)}=\int_{0}^{\infty} d \tilde{t} \chi(\tilde{t}) e^{i(\omega+i \epsilon) \tilde{t}} .
$$




\subsection{Response functions and interactions between modes}

The convergence factor $i \epsilon$ has to be send to zero after the imaginary or the real part of $\chi(\omega)$ is taken. From Eq. 8.51, we see that $\operatorname{Im}[\chi(\omega)]$ yields the out-of-phase response.

We are interested in the time average of the energy absorption rate. Plugging Eq. 8.51 into Eq. 8.49 and averaging over one period $\tau=1 / \nu$ yields

$$
\frac{1}{\tau} \int_{0}^{\tau} \frac{d E}{d t}=\frac{\delta_{0}^{2} \omega}{2} \operatorname{Im}[\chi(\omega)] .
$$

The mean energy absorption rate is therefore proportional to the imaginary part of the Fourier transformation of the dynamical susceptibility and proportional to the modulation frequency.

Thus, the total energy increase $\Delta E(\omega)$ after a modulation time $T_{\text {mod }}$ is

$$
\Delta E(\omega)=\frac{\delta_{0}^{2} \omega T_{\bmod }}{2} \operatorname{Im}[\chi(\omega)]
$$

Experimentally, we set $T_{\bmod }=n_{\text {mod }} \tau$, i.e., we set the modulation time to an integer multiply $n_{\text {mod }}$ of the oscillation period. The fixed number of oscillation-cycles has the advantage that the explicit $\omega$-factor in the previous formula cancels:

$$
\Delta E(\omega)=\pi \delta_{0}^{2} n_{\text {mod }} \operatorname{Im}[\chi(\omega)] .
$$

The absorbed energy is therefore directly proportional to the imaginary part of the fourier transform of the dynamical susceptibility $\operatorname{Im}[\chi(\omega)]$. In the experiment, we actually observed the temperature increase of the system. For small $\Delta E$, we expect the temperature increase to be directly proportional to the energy increase.

We now turn to the form of $\chi(\tilde{t})$. The Kubo formula states that

$$
\chi(\tilde{t})=\frac{i}{\hbar}\langle[\hat{K}(\tilde{t}), \hat{K}(0)]\rangle \Theta(\tilde{t})
$$

where $\hat{K}(\tilde{t})=e^{\frac{i}{\hbar} \hat{H}_{B H} \tilde{t}} \hat{H} e^{-\frac{i}{\hbar} \hat{H}_{B H} \tilde{t}}$ describes the time dependence in the Heisenberg picture, $[\hat{K}(\tilde{t}), \hat{K}(0)]=\hat{K}(\tilde{t}) \hat{K}(0)-\hat{K}(0) \hat{K}(\tilde{t})$, and $\langle\ldots\rangle$ denotes the quantum mechanical avarage in the equilibrium system without perturbation (see, e.g., Refs. [20, 152]). The key property of this formula is that it relates a response function to a correlation function of the thermal equilibrium system.

With the Kubo formula, one can derive the so-called fluctuation-dissipation theorem ${ }^{1}$

$$
\operatorname{Im}[\chi(\omega)]=\frac{1}{2 \hbar}\left(1-e^{-\beta \hbar \omega}\right) S(\omega),
$$

\footnotetext{
${ }^{1}$ depening on the author, also Eq. 8.54 is sometimes called fluctuation-dissipation theorem
} 
where $S(\omega)$ can be written in a spectral decomposition as

$$
S(\omega)=2 \pi \sum_{i, f} \frac{e^{-\beta E_{i}}}{Z}|\langle f|\hat{K}| i\rangle|^{2} \delta\left(\hbar \omega-\left(E_{f}-E_{i}\right)\right) .
$$

Here $|i\rangle$ are eigenstates of $\hat{H}_{B H}$ with eigenenergy $E_{i}, \delta(x)$ denotes the Delta-function, and $\beta=1 /\left(k_{\mathrm{B}} T\right)$ with $T$ the temperature of the system. The interpretation of these formulas is that $S(\omega)$ and thus $\operatorname{Im}[\chi(\omega)]$ yield information on the excited-state spectrum of the equilibrium system. Only if there is a pair of eigenstates $i, j$ with an energy difference that matches the modulation frequency an excitation is possible. Furthermore, there are additional weight-factors; namely, the transition-matrix elements $|\langle f|\hat{K}| i\rangle|$ and the thermal occupation $\frac{e^{-\beta E_{i}}}{Z}$ of the initial state $i$.

Lattice modulation spectroscopy therefore accesses the excited state spectrum of the system. Note that lattice modulation is spatially uniform and therefore couples to modes with zero momentum. Within a mean-field approximation, lattice modulation for the Bose-Hubbard has been studied in Ref. [49] yielding a delta-function peak at the frequency of the Higgs amplitude mode. The mean-field approximation, however, misses any broadening of the peak due to decay of the Higgs mode into lower-frequency phase modes. An approximate description in terms of the quantum rotor model (Eq.7.24) yielded a low-energy $\omega^{3}$ increase of the response additionally to a delta-function peak [145]. A recent Quantum Monte Carlo (QMC) study investigated the experimentally relevant situation of lattice modulation for the two-dimensional Bose-Hubbard model [41]. It showed a peaked response close to the quantum phase transition. Additional, the onset of the response also survived at finite temperatures and trapping parameters similar to our experiment. 


\subsubsection{Visibility of the Higgs mode in the two-dimensional $O(2)$ model}

\section{Scalar and longitudinal response functions}

Within $O(2)$ theory, lattice depth modulation, to lowest order, leads to a perturbation

$$
\begin{aligned}
\mathcal{L}(t) & =\mathcal{L}+\delta(t)|\Psi|^{2} \\
\delta(t) & =\delta_{0} \cos (\omega t)
\end{aligned}
$$

Thus, lattice modulation couples to the order parameter in a rotationally symmetric fashion. Linear response theory can be formulated in the same way as described in the previous section for the Bose-Hubbard model. The response function $\chi_{s}(\omega)$ for coupling to $|\Psi|^{2}$ is called the scalar response function [40]. The energy absorption is again proportional to the imaginary part of $\chi_{s}(\omega)$.

This should be contrasted to the longitudinal response function $\chi_{l}(\omega)$ for coupling to the longitudinal component of $\Psi$. Choosing $\Psi_{0}$ along the real axis, the longitudinal component would be the real part of $\Psi$. This coupling is not rotationally symmetric. The longitudinal response function is, e.g., measured using neutron scattering on quantum magnets [20, 141]. The low-energy description for these systems is based on a three-dimensional $O(3)$ model, an extension of the $O(2)$ model to a three-component order parameter. Additionally, neutron scattering allows for the detection of the transverse response functions that describes coupling to one of the components perpendicular to $\Psi_{0}$.

\section{Visibility of the Higgs mode in two dimensions}

Until recently, longitudinal and transverse response functions have been in the focus of theoretical investigations [20,36-38]. The longitudinal response function of an $O(N)$ theory in dimensions $d>2$ is expected to show a resonance that is attributed to a Higgs amplitude mode. Indeed, a peak that shows softening at a quantum phase transition was observed in the previously mentioned experiment on quantum magnets [141] described by a three-dimensional theory [20].

For $d=2$, the results for the longitudinal response function indicated that the Higgs mode is over-damped and therefore no resonance in the response function is observable. However, recent theoretical investigations of $O(N)$ theories suggested that the visibility of the Higgs mode in two dimensions depends on whether the longitudinal or the scalar response is probed [39, 40,42]. In particular, it is was argued that a resonance should be observable in a scalar response function for $d=2[40,42]$, which is also indicated by the previously mentioned QMC study of lattice modulation for the Bose-Hubbard model [41].

The damping of the Higgs mode results from the interacting part of the Lagrangian. 


\section{Introduction to amplitude and phase modes}

The complete decomposition into free and interacting Lagrangian reads

$$
\begin{aligned}
\mathcal{L} & \propto \mathcal{L}_{\text {free }}+\mathcal{L}_{\text {int }} \\
\mathcal{L}_{\text {free }} & =c^{2} \partial_{\nu} \phi_{1} \partial^{v} \phi_{1}-2|r| \phi_{1}^{2}+c^{2} \partial_{\nu} \phi_{2} \partial^{v} \phi_{2} \\
\mathcal{L}_{\text {int }} & =-u\left(4 \sqrt{\frac{|r|}{2 u}} \phi_{1} \phi_{2}^{2}+2 \phi_{1}^{2} \phi_{2}^{2}+\phi_{2}^{4}+4 \sqrt{\frac{|r|}{2 u}} \phi_{1}^{3}+\phi_{1}^{4}\right) .
\end{aligned}
$$

The non-interacting part is independent of $u$, which allows for a perturbative calculation in orders of $u$. The leading contribution to the interacting part $\mathcal{L}_{\text {int }}$ is the term proportional to $\phi_{1} \phi_{2}^{2}$. It describes the decay of a Higgs mode into two Nambu-Goldstone modes.

In the following, we will briefly describe how the $\phi_{1} \phi_{2}^{2}$ term influences the longitudinal response function $\chi_{l}(\omega, k)$ for a frequency $\omega$ and momentum $k$. We introduce the momentum dependence because the calculation at zero-momentum is inconvenient to formulate. The linear response formalism introduced in the previous subsection can be extended to finite momentum by considering a modulation of the form $\cos (\omega t-k x)$, where $k$ and $x$ are vectors. For the derivation, we follow Refs. [20, 40].

The response function for the free Lagrangian is

$$
\begin{aligned}
\chi_{l, 0}(\omega, k) & =\frac{1}{c^{2} k^{2}-(\omega+i \epsilon)^{2}+\Delta_{0}^{2}} \\
& =\frac{1}{\tilde{k}^{2}+\Delta_{0}^{2}} .
\end{aligned}
$$

In the second line, we introduced $\tilde{k}^{2}=c^{2} k^{2}-(\omega+i \epsilon)^{2}$ to simplify the notation. The energy absorption is proportional to the imaginary part of $\chi_{l, 0}(\omega, k)$ in the limit of $\epsilon$ going to zero. This yields a delta-function peak centered at frequency $\omega^{2}=\Delta_{0}^{2}+c^{2} k^{2}$. Thus, we find a sharply peaked response for the energy absorption at the frequency for the Higgs mode if we ignore interactions between modes.

Including contributions from interactions, the longitudinal response can be written as

$$
\chi_{l}(\tilde{k})=\frac{1}{\tilde{k}^{2}+\Delta_{0}^{2}-\Sigma_{l}(\tilde{k})},
$$

where $\Sigma_{l}(\omega)$ is the self energy. To lowest order in $u$, one has

$$
\begin{aligned}
& \Sigma_{l}(\tilde{k})=4|r| u \Pi(\tilde{k}), \\
& \Pi(\tilde{k})=\int \frac{d^{d+1} p}{(2 \pi)^{d+1}} \frac{1}{p^{2}(p+\tilde{k})^{2}},
\end{aligned}
$$


where $d$ is the number of dimensions. Expanding $\chi_{l}(\tilde{k})$ in $u$ yields

$$
\chi_{l}(\tilde{k})=\frac{1}{\tilde{k}^{2}-\Delta_{0}^{2}}+\frac{4|r| u}{\left(\tilde{k}^{2}-\Delta_{0}^{2}\right)^{2}} \Pi(\tilde{k}) .
$$

Thus, to lowest order in $u$, the correction depends on the integral $\Pi(\tilde{k})$ and therefore on the dimensionality of the system.

One can solve the integral and calculate the imaginary part of Eq.8.69. This yields

$$
\operatorname{Im}\left[\chi_{l}(\omega, 0)\right] \propto \frac{1}{\omega}
$$

for low frequencies and zero-momentum in a two-dimensional system. In contrast, the calculation for a three-dimensional system yields a constant response at low frequencies. In both cases the delta function peak at $\omega=\Delta_{0}$ survives at higher frequencies. These results give a hint that the calculation of response functions for the two-dimensional case is difficult because already to lowest order in $u$ the free result is strongly modified by low-frequency divergence.

A more elaborate calculation can be performed using a so-called $\frac{1}{N}$-expansion, where $N$ is the number of components of the order parameter. The $N \rightarrow \infty$ limit yields that the Higgs peak in $\operatorname{Im}\left[\chi_{l}(\omega, 0)\right]$ completely disappears in favor of the low-frequency $\frac{1}{\omega}$ divergence in a two-dimensional system. A more recent calculation [42] extended these results to first order in $\frac{1}{N}$. The low frequency divergence is still present; however, $\chi_{l}(\omega, 0)$ acquires a small oscillatory component. As a consequence, a broad peak structure is visible if one plots $\omega \operatorname{Im}\left[\chi_{l}(\omega, 0)\right]$, where the multiplication with $\omega$ suppresses the low-frequency divergence.

The previously stated results concern the longitudinal response function. In our experiment, we expect to measure the scalar response. The scalar response function has the feature that it suppresses the low-energy divergence by a factor of $\omega^{4}$, thus leading to a characteristic $\omega^{3}$ behavior at low-frequencies. This result is observed in a weak coupling-expansion in $u$, and in a $\frac{1}{N}$-expansion (for $N \rightarrow \infty$ and up to first order in $\left.\frac{1}{N}\right)[40,42]$. It can be understood when writing the Lagrangian in a polar decomposition $\Psi=\left(\Psi_{0}+h\right) e^{i \phi}$. Now, a Higgs mode is associated with oscillations of $h$ and Nambu-Goldstone modes are associated with oscillations of $\phi$. In this decomposition, lattice modulation of $|\Psi|^{2}$ couples only to $h$. In contrast, in the previously used decomposition, a modulation of $|\Psi|^{2}$ also couples to $\phi_{2}$. The polar decomposition is therefore better suited for a calculation of the scalar response. We do not give the full polar decomposition of the Lagrangian here. The crucial point is that the leading order term in the interacting part is proportional to $h \partial_{\nu} \phi \partial^{v} \phi$, in contrast to $\phi_{1} \phi_{2}^{2}$. The additional derivatives lead to the mentioned $\omega^{4}$ suppression [42].

The question remains whether or not a Higgs resonance is visible in the scalar re- 


\section{Introduction to amplitude and phase modes}

sponse function. The $\frac{1}{N}$-expansion in the $N \rightarrow \infty$ limit yielded a peaked scalar response function [42]

$$
F(\omega)=\operatorname{Im}\left[\chi_{l}(\omega, 0)\right] \propto \frac{\omega^{3}}{\left(\omega^{2}-\Delta_{0}^{2}\right)^{2}+4 \gamma^{2} \omega^{2}}
$$

that we also used in the analysis of the experimental data (Sec. 9.4.3). The function shows a broadened peak with a characteristic width $\gamma$. However, $\gamma$ stays finite when approaching the critical point, which is inconsistent with a universal scaling law for $\operatorname{Im}\left[\chi_{l}(\omega, 0)\right]$ (see Sec. 9.4.2 and 9.4.3). A more refined calculation including first order in $\frac{1}{N}$ yielded a response function that does fulfill the universal scaling law and shows a resonance that is attributed to a Higgs amplitude mode [42]. In conclusion, these recent results, in combination with the mentioned QMC study [41], indicate that a resonance in the scalar response function close to the phase transition of the twodimensional $O(2)$ model exists and that the Higgs mode is therefore not overdamped. 


\section{Detection of the Higgs amplitude mode at the 2d SF-Mott-insulator transition}

We now turn to the experimental detection of the Higgs amplitude mode in a strongly interacting superfluid close to the two-dimensional superfluid-Mott-insulator transition. The general approach is to use the sensitive in situ temperature measurement described in Sec. 4.3 to probe the response of the system to lattice modulation with varying frequency.

\subsection{Experimental procedure}

Our experiment began with the preparation of a two-dimensional degenerate gas of ${ }^{87} \mathrm{Rb}$ atoms in a single anti-node of an optical standing wave (Sec. 3). To realize different couplings $j=J / U$, we loaded the two-dimensional gas into a square optical lattice with variable depth $V_{0}$ using an s-shaped ramp with a total duration of $120 \mathrm{~ms}$ (Fig. 9.1). Our systems contained an atom number of 190(36) resulting in a central density close to one atom per lattice site for typical radial trapping frequencies of $\omega /(2 \pi) \approx 60 \mathrm{~Hz}$.

We then modulated the lattice depth with an amplitude of $3 \%$ at variable frequencies $v_{\text {mod }}$. The modulation time was set to 20 oscillation-cycles, thus avoiding an unwanted enhanced response at higher frequencies present in experiments with fixed modulation time [32, 33] (Sec. 8.6). We allowed for an additional hold time, keeping the sum of modulation and hold time constant at $200 \mathrm{~ms}$. The additional hold time had two purposes. First, it allowed the system to relax after excitations were created by the modulation. Second, we observed a small heating of the system when holding it at a constant $j$ without modulation. With the additional hold time, the total time that the system spent at a given $j$ was the same for all modulation frequencies, thus resulting in the same absolute increase of temperature due to the intrinsic heating. To quantify the response, we adiabatically increased the lattice depth to reach the atomic limit $(j \approx 0)$ and measured the temperature of the system with the scheme described in Sec.4.3. It is the high sensitivity of this method which allowed us to reduce the modulation amplitude by almost an order of magnitude compared to earlier experiments $[32,33]$ and to stay well within the linear response regime (Sec. 9.3).

We then took traces of the temperature response as a function of the modulation frequency $v_{\text {mod }}$ for different lattice depths $V_{0}$, corresponding to different $j$ values. 


\section{Detection of the Higgs amplitude mode at the 2d SF-Mott-insulator transition}

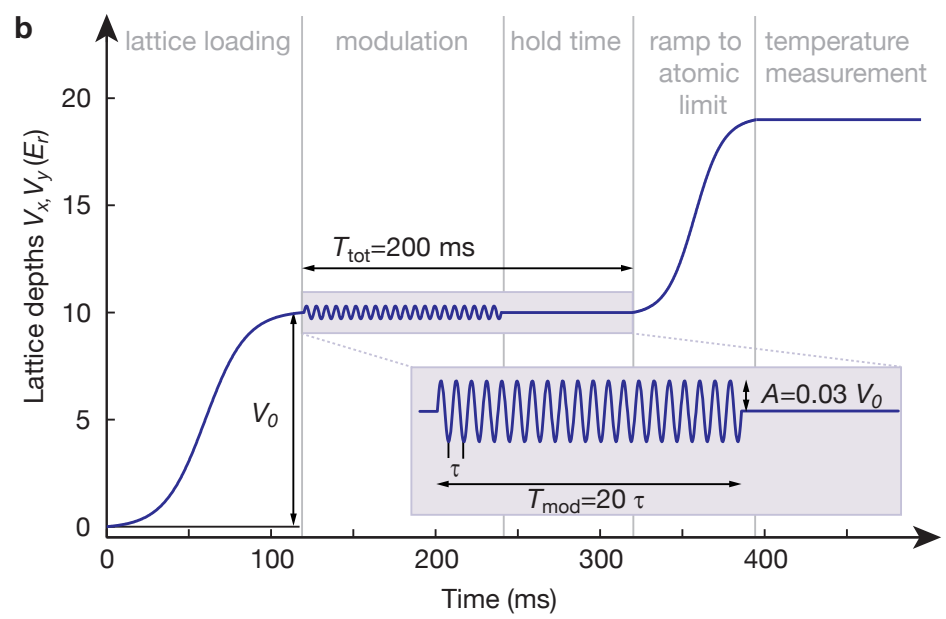

Figure 9.1.: Experimental sequence for the detection of the amplitude Higgs mode. Figure shows the depth of the horizontal lattices $V_{x}, V_{y}$ (see Sec.3) during the sequence. Both lattice depths follow the same sequence. For details, see text.

\subsection{Softening of the mode gap}

The results for selected lattice depths $V_{0}$ are shown in Fig. 9.2b. The corresponding $j$ values are such that the system is close to the superfluid-Mott-insulator transition, but still on the superfluid side. We observe a gapped response with an asymmetric overall shape that will be analysed in Sec.9.4. Notably, the maximum observed temperature after modulation is well below the 'melting' temperature for a Mott insulator in the atomic limit $T_{\text {melt }} \approx 0.2 U / k_{B}$ [54].

To obtain numerical values for the onset of spectral response, we fitted each spectrum with an error function centered at a frequency $v_{0}$ (solid black lines in Fig. 9.2b). With $j$ approaching $j_{c}$, we observe a pronounced shift of the gap to lower frequencies that is already visible in the raw data (Fig.9.2b and Fig.9.9a) and becomes even more apparent for the fitted gap $v_{0}$ as a function of $j / j_{c}$ (Fig.9.2a, filled circles). The $v_{0}$ values are in quantitative agreement with a prediction for the Higgs gap at commensurate filling $h v_{\mathrm{SF}} / U=\left[(3 \sqrt{2}-4)\left(1+j / j_{c}\right)\right]^{1 / 2}\left(j / j_{c}-1\right)^{1 / 2}$ (solid line) based on an analysis of variations around a mean field state $[49,138]$. Throughout this thesis, we rescaled $j_{c}$ in the theoretical calculations to match the value $j_{c} \simeq 0.06$ obtained from QMC simulations [56]. We interpret the observed shift to lower frequencies as the softening of the Higgs amplitude mode described in Sec. 8.2.4.

The complete fitting function was $T=T_{0}+\Delta T / 2\left\{\operatorname{erf}\left[\frac{1}{\sigma_{e}}\left(\nu_{\bmod }-v_{0}\right)\right]+1\right\}$, where $\operatorname{erf}(x)$ denotes the error function. The fitting parameters were the temperature offset $T_{0}$, the temperature increase $\Delta T$, the width $\sigma_{e}$ and the center frequency $v_{0}$. The 

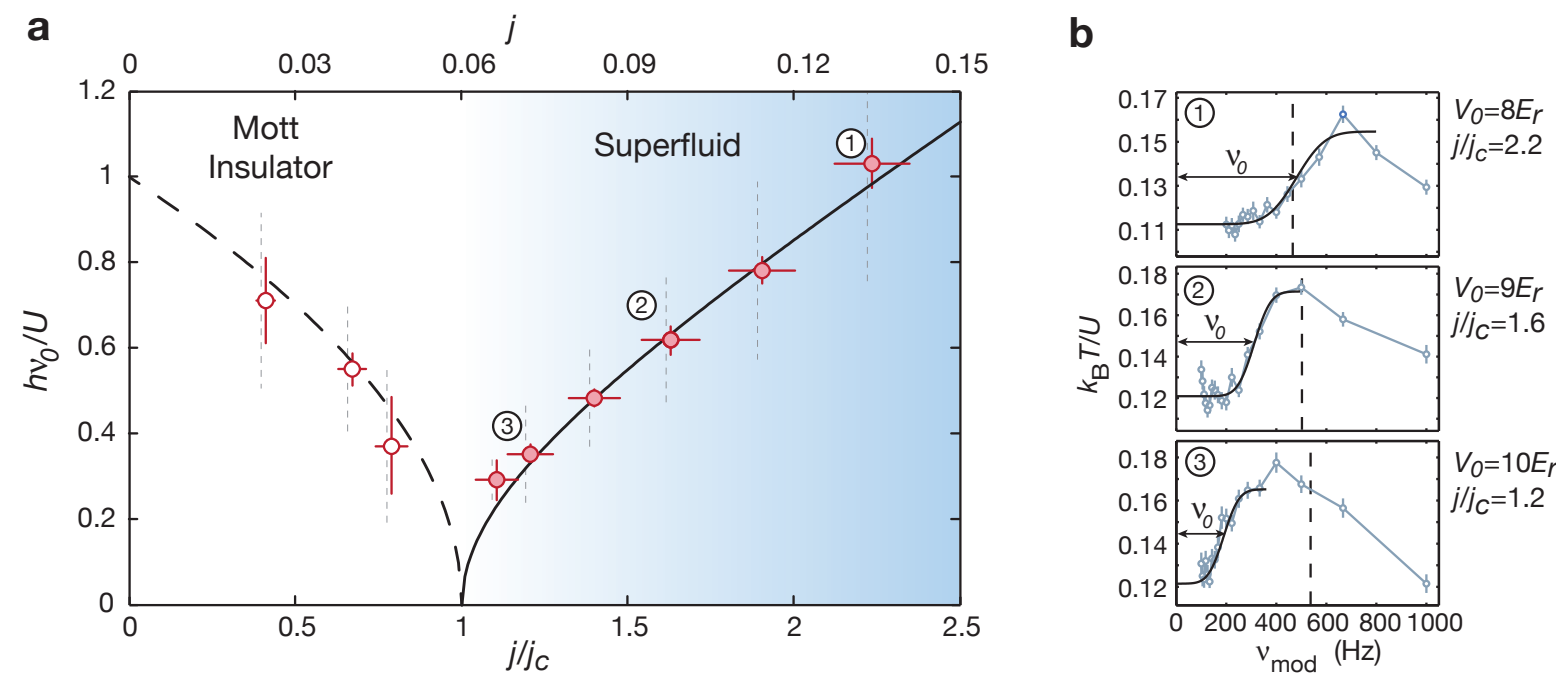

Figure 9.2.: Softening of the Higgs mode. a, The fitted gap values $h v_{0} / U$ (circles) show a characteristic softening close to the critical point in quantitative agreement with analytic predictions for the Higgs and the Mott gap (solid line and dashed line, see text). Horizontal and vertical errorbars denote the experimental uncertainty of the lattice depths and the fit error for the center frequency of the error function, respectively (see text). Vertical dashed lines denote the width of the fitted error function and characterize the sharpness of the spectral onset. The blue shading highlights the superfluid region. $\mathbf{b}$, Temperature response to lattice modulation (circles and connecting blue line) and fit with an error function (solid black line) for three different points in a labeled by corresponding numbers. With the coupling $j$ approaching the critical value $j_{c}$, the change of the gap values to lower frequencies is clearly visible (from panel 1 to 3 ). Vertical dashed lines mark the frequency $U / h$ corresponding to the on-site interaction. Each data point results from an average of the temperatures over $\approx 50$ experimental runs. Error bars denote the standard error of the mean (s.e.m.) resulting from this average.

fit function is a model free approach to extract numerical values for the onset of spectral response. The center frequency $v_{0}$ (circles in Fig. 9.2a) is a measure for the position of the spectral onset, while the width $\sigma_{e}$ is a measure for its sharpness. The width $\sigma_{e}$ can also be seen as an estimation for the maximum error on the extraction of the position of the onset (vertical dashed lines in Fig. 9.2a). During the least-square optimization, data points at frequencies larger than $v_{0}+2.5 \sigma_{e}$ were excluded. The vertical errorbar in Fig. 9.2a is given by the $1 \sigma$ fitting error for $v_{o}$ and the vertical dashed lines denote $\pm \sigma_{e}$.

As discussed, the sharpness of the spectral onset can be quantified by the width of the fitted error function, which is separately shown in Fig. 9.3a. Approaching the 


\section{Detection of the Higgs amplitude mode at the 2d SF-Mott-insulator transition}
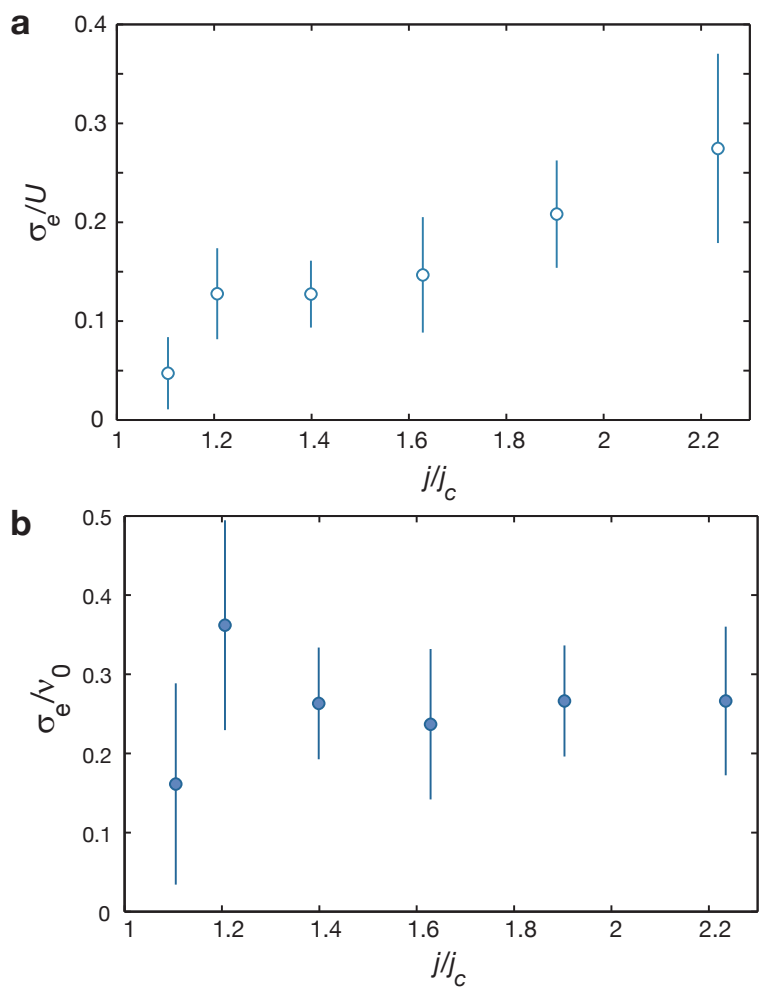

Figure 9.3.: Width of the model function. a, Width $\sigma_{e}$ of the fitted error function for $j>j_{c}$. This is the same data as shown in Fig. 9.2a as vertical dashed lines. b. Width $\sigma_{e}$ divided by the center frequency $v_{0}$ of the fitted error function for $j>j_{c}$. The data show that the onset of the spectral response on an absolute scale becomes sharper approaching the critical point, while the width normalized with the center frequency of the onset stays approximately constant. Error bars denote the fit error.

critical point, the spectral onset becomes sharper, while the width $\sigma_{e}$ normalized to the center frequency $v_{0}$ remains constant (Fig. 9.3b). This shows that the width of the spectral onset scales in the same way with the distance to the critical point as the gap frequency.

We observe similar gapped responses in the Mott-insulating regime (Figs.9.4 and 9.9), with the gap closing continuously when approaching the critical point (Fig. 9.2a, open circles). We interpret this as a result of combined particle and hole excitations with a frequency given by the Mott excitation gap that closes at the transition point (see discussion in Sec. 2.2). The fitted gaps are consistent with the Mott gap $h v_{\mathrm{MI}} / U=\left[1+(12 \sqrt{2}-17) j / j_{c}\right]^{1 / 2}\left(1-j / j_{c}\right)^{1 / 2}$ predicted by mean field theory [49] (dashed line in Fig. 9.2a). Note that $v_{\mathrm{MI}}$ is a mean-field approximation of the full width of the Mott-insulating lobe $\Delta_{M I}$ as defined in Sec. 2. 


\subsection{Raw data and linear response}

In Fig. 9.4 we show the raw data for all data points plotted in Fig. 9.2a. The closing of the gap coming from the superfluid side of the transition $\left(j / j_{c}>1\right)$ is clearly visible and is followed by a reopening in the Mott-insulating regime $\left(j / j_{c}<1\right)$.

We probed the time dependence of the response for two different combinations of

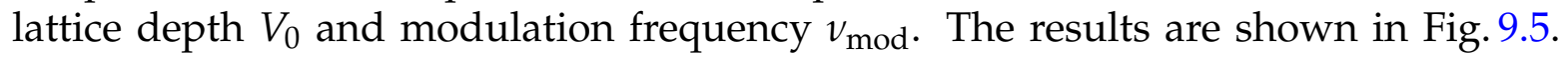
A linear response as a function of the number of oscillation cycles is visible up to 40 modulation cycles. Notably, our experiments were performed at 20 cycles, staying well within the regime of linear temperature response. A linear fit yielded a slope of $1.8(2) \cdot 10^{-3} \frac{k_{B} T}{U} \frac{1}{\tau}$ in both cases, where $\tau$ is the time for a single cycle of the modulation.
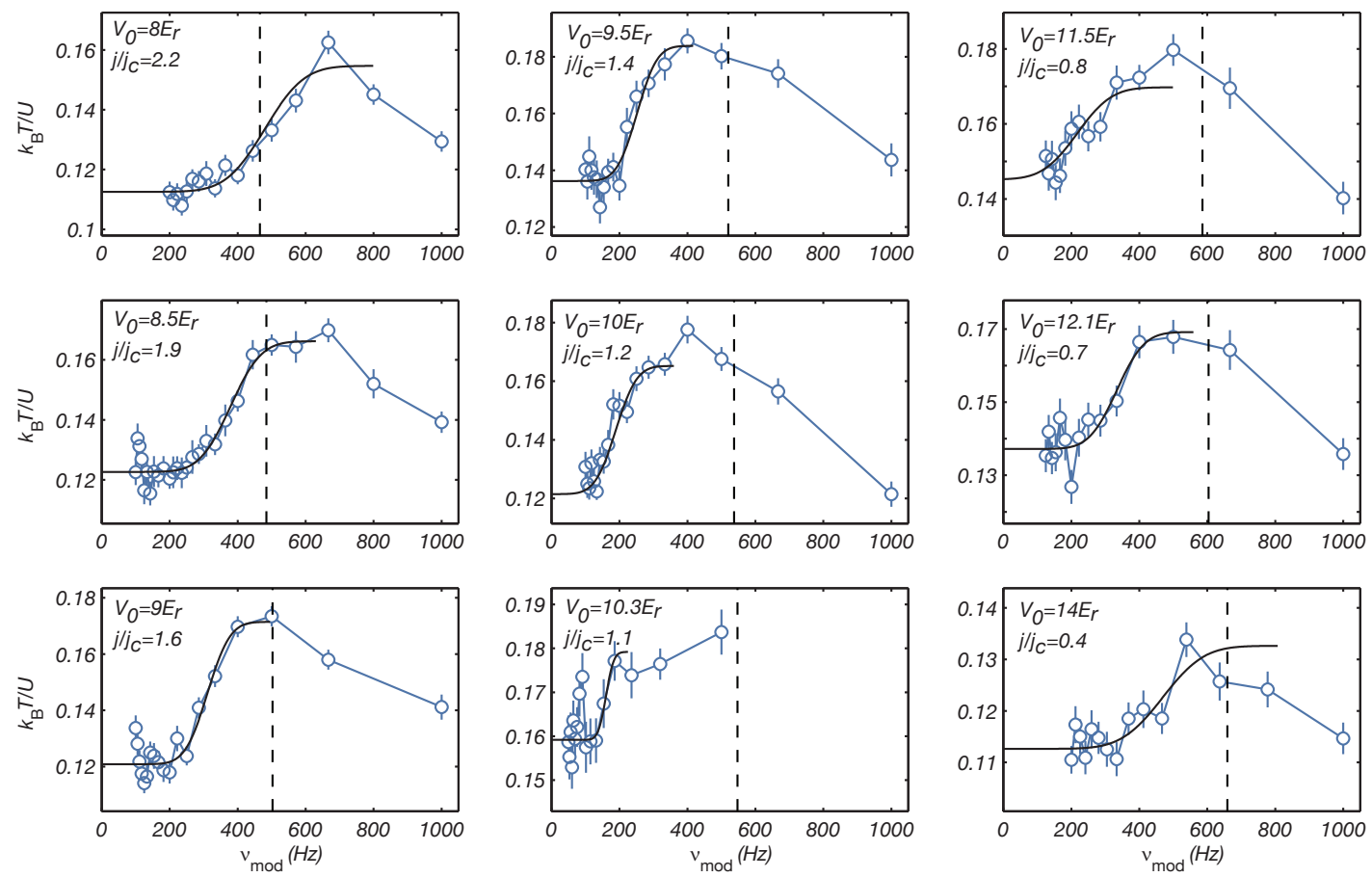

Figure 9.4.: Raw data and fits for all data points shown in Fig. 9.2. Each data point results from an average of the temperatures from about 50 experimental runs. Dashed line indicates the value of the on-site interaction $U$. Error bars, s.e.m. 

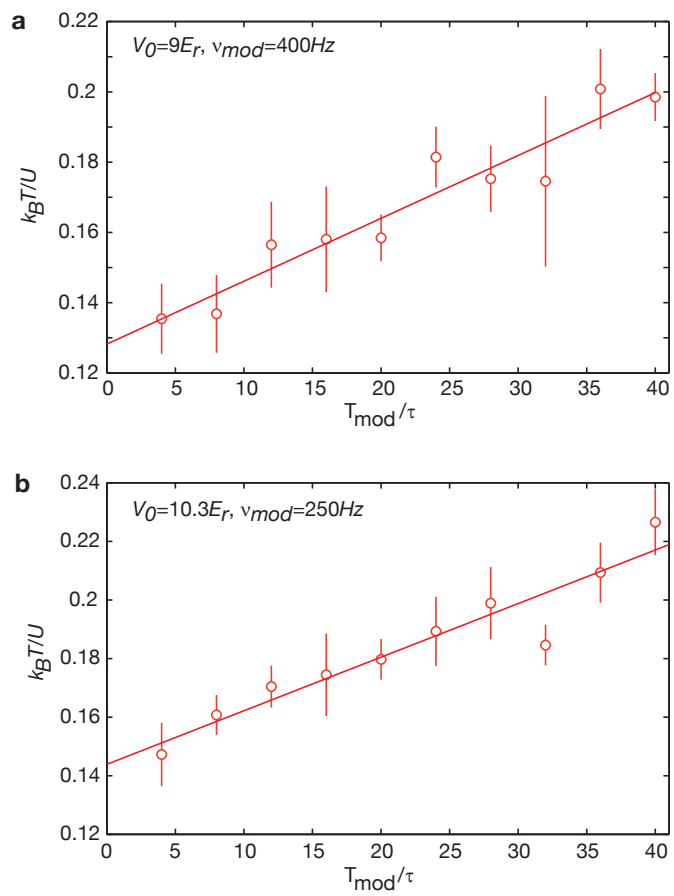

Figure 9.5.: Linear Response. Temperature as a function of the number of modulation cycles for $\mathbf{a}, V_{0}=9 E_{r}$ and $v_{\text {mod }}=400 \mathrm{~Hz}$ and $\mathbf{b}, V_{0}=10.3 E_{r}$ and $v_{\text {mod }}=250 \mathrm{~Hz}$. Error bars, s.e.m.

\subsection{Analysis of the response}

\subsubsection{Gutzwiller analysis}

The observed softening of the onset of spectral response in the superfluid regime has led to an identification with collective excitations of Higgs type. To gain further insight into the full in-trap response, we calculated the eigenspectrum of the system in a Gutzwiller approach $[49,147]$ (see Sec. 9.8). The result is a series of discrete eigenfrequencies (Fig. 9.6a) and the corresponding eigenmodes show in-trap superfluid density distributions, which are reminiscent of the vibrational modes of a drum (Fig. 9.6b). The frequency of the lowest-lying amplitude-like eigenmode closely follows the long-wavelength prediction for homogeneous commensurate filling $v_{S F}$ over a wide range of couplings $j / j_{c}$ until the response rounds off in the vicinity of the critical point due to the finite size of the system (Fig. 9.6b). Fitting the low-frequency edge of the experimental data can be interpreted as extracting the frequency of this mode, which explains the good quantitative agreement with the prediction for the homogeneous commensurate filling in Fig. 9.2a. Amplitude-like modes at different frequencies from the lowest-lying amplitude-like mode broaden the spectrum only above the onset of spectral response.

Lattice modulation might also couple to phase-like modes that are, however, at lower 

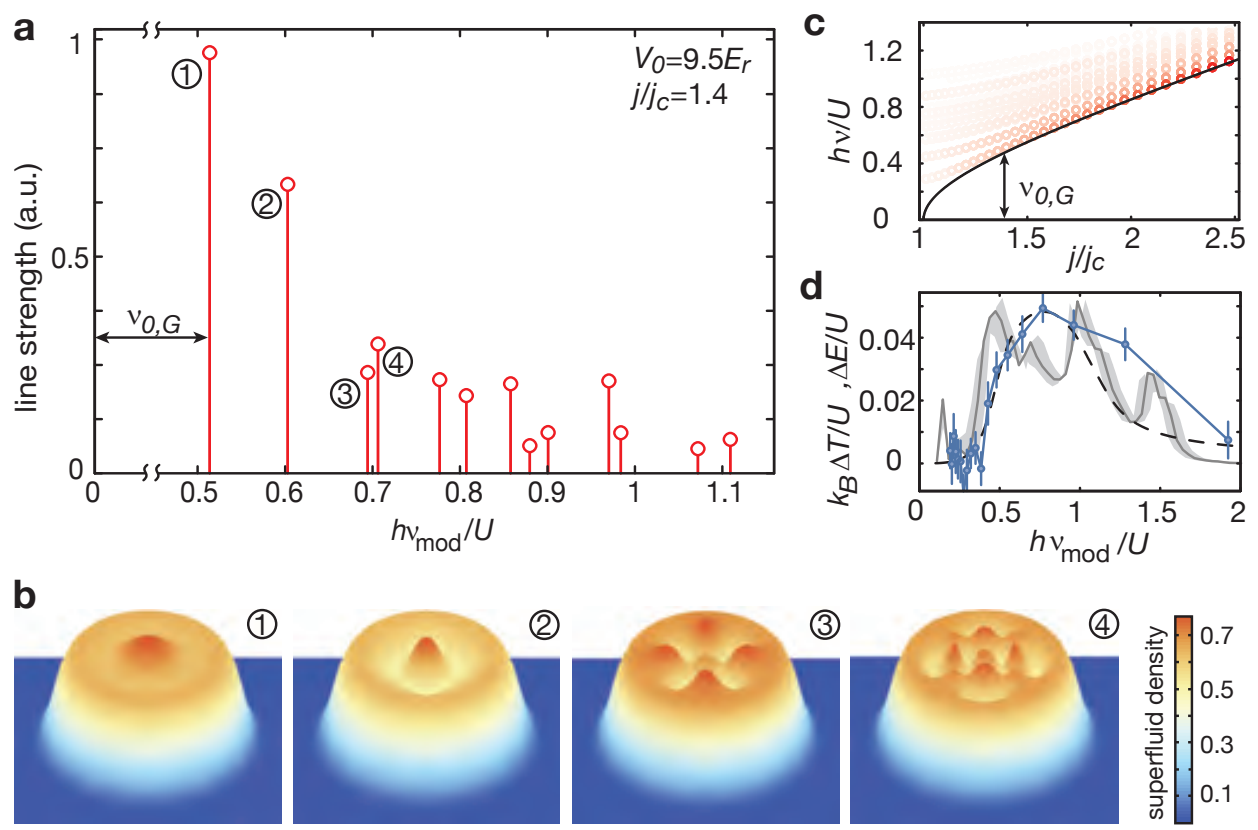

Figure 9.6.: Theory for in-trap response. a, A diagonalization of the trapped system in a Gutzwiller approximation shows a discrete spectrum of amplitude-like eigenmodes. Shown on the vertical axis is the strength of the response to a modulation of $j$. Eigenmodes of phasetype are not shown (see Sec.9.9) and $v_{0, G}$ denotes the gap as calculated in the Gutzwiller approximation. $\mathbf{b}$, In-trap superfluid density distribution for the four amplitude modes with lowest frequency marked by corresponding numbers in a. In contrast to the superfluid density, the total density of the system stays almost constant (not shown). c, Discrete amplitude mode spectrum for various couplings $j / j_{c}$. Each red circle corresponds to a single eigenmode with the intensity of the color being proportional to the line strength. The gap frequency of the lowest-lying mode follows the prediction for commensurate filling (solid line, same as in Fig. 9.2a) until a rounding off takes place close to the critical point due to the finite size of the system. d, Comparison of the experimental response at $V_{0}=9.5 E_{r}$ (blue circles and connecting blue line; error bars, s.e.m.) with a $2 \times 2$ cluster mean field simulation (gray line and shaded area) and a heuristic model (dashed line, for details see text). The simulation was done for $V_{0}=9.5 E_{r}$ (gray line) and for $V_{0}=(1 \pm 2 \%) \cdot 9.5 E_{r}$ (shaded gray area) in order to account for the experimental uncertainty of the lattice depth and predicts the energy absorption per particle $\Delta E$.

frequencies and separated from the amplitude mode response by a gap. A careful analysis of phase-like modes is presented in Sec. 9.9. Additionally, we speak of amplitude-like and phase-like modes, because the Gutzwiller analysis shows that the corresponding trajectories in the complex plane are ellipses with their main axis in amplitude or phase direction, respectively (Sec. 9.9). 


\section{Detection of the Higgs amplitude mode at the $2 \mathrm{~d}$ SF-Mott-insulator transition}

\subsubsection{Scaling of the low-frequency response}

The previous eigenmode analysis does not yield any information about the finite spectral width of the modes, which stems from the interaction between amplitude and phase excitations (see also Sec. 8.6). We will consider the question of the spectral width by analysing the low-, intermediate- and high-frequency part of the response separately.

We begin by examining the low-frequency part of the response. As discussed in Sec. 8.6, the response of a strongly interacting two-dimensional superfluid is expected to diverge at low frequencies, if the probe in use couples longitudinally to the order parameter $[20,37,38,42]$ (e.g., to the real part of $\Psi$, if $\Psi$ was chosen along the real axis), as it is the case for neutron scattering. If, instead, the coupling occurs in a rotationally invariant fashion (i.e., to $|\Psi|^{2}$ ), as expected for lattice modulation, such a divergence could be avoided [36, 40, 42, 145].

In this case, the amplitude mode response at low frequencies is expected to be proportional to $v^{3}$, which is observed in a weak-coupling expansion [40], in a large $N$ expansion [36, 40,42] and in the quantum phase model [145]. Additionally, dimensional analysis shows that the amplitude mode response for an $O(N)$ field theory should follow a scaling of the form [20,40,42] $F\left(v, \frac{j}{j_{c}}\right)_{u}=A \Delta^{3-2 / v_{c}} \Phi\left(\frac{v}{\Delta}\right)$, where $\Delta \propto\left(1-\frac{j}{j_{c}}\right) v_{c}$ is a typical energy scale, $v_{c}$ is the critical exponent associated with $\Delta$, $A$ is a constant and $\Phi$ a universal function. Combining this scaling with the $v^{3}$ prediction yields $F\left(v, \frac{j}{j_{c}}\right)_{u}=A\left(1-\frac{j}{j_{c}}\right)^{-2} v^{3}$ at low frequencies [42]. The experimentally observed signal is consistent with this scaling at the 'base' of the absorption feature (Fig. 9.7). This indicates that the low-frequency part is dominated by only a few intrap eigenmodes that approximately show the generic scaling of the homogeneous system for a response function describing coupling to $|\Psi|^{2}$.

The full scaling $F\left(v, \frac{j}{j_{c}}\right)_{u}=A \Delta^{3-2 / v_{c}} \Phi\left(\frac{v}{\Delta}\right)$ predicts that the full shape of the response function in $v$ direction is rescaled when approaching the critical point. This excludes a different scaling of width and position of the response. Our data is consistent with this because the fitted width of the onset of the response $\sigma_{e}$ scales approximately in the same way as the fitted gap value $v_{0}$ (Fig. 9.3). 


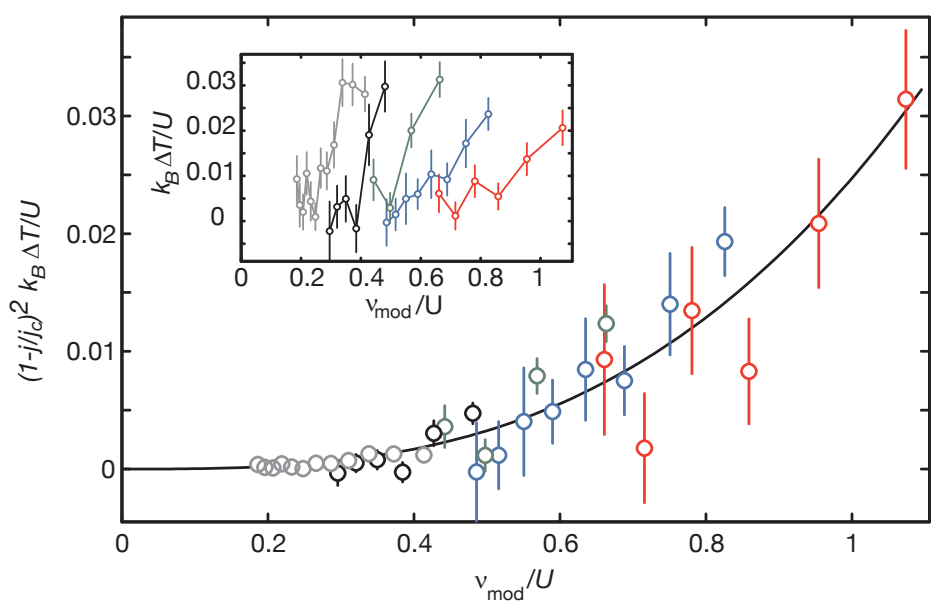

Figure 9.7.: Scaling of the low-frequency response. The low-frequency response in the superfluid regime shows a scaling compatible with the prediction $\left(1-j / j_{c}\right)^{-2} v^{3}$ (see text). Shown is the temperature response rescaled with $\left(1-j / j_{c}\right)^{2}$ for $V_{0}=10 E_{r}$ (grey), 9.5 $E_{r}$ (black), $9 E_{r}$ (green), $8.5 E_{r}$ (blue), $8 E_{r}$ (red) as a function of the modulation frequency. The black line is a fit of the form $a v^{b}$, with a fitted exponent $b=2.9(5)$. The inset shows the same data points without rescaling for comparison. For this figure, we chose frequencies in a span from $v_{0}-1.5 \sigma_{e}$ to $v_{0}+0.5 \sigma_{e}$, with $v_{0}$ and $\sigma_{e}$ taken from the error function fit of the individual responses.

\subsubsection{Intermediate frequencies and heuristic model}

In the intermediate-frequency regime, it remains a challenge to construct a firstprinciple analytical treatment of the in-trap system including all relevant decay and coupling processes. Lacking such a theory, we constructed a heuristic model combining the discrete spectrum from the Gutzwiller approach (Fig. 9.6a) with the lineshape for a homogeneous system based on an $O(N)$ field theory in two dimensions, calculated in the large $N$ limit $[36,40]$.

For a given $j / j_{c}$ value, the Gutzwiller approach yields a series of amplitude-like normal modes with frequencies $v_{i}$ and corresponding line strengths $S_{i}$. The heuristic model consists of summing up a response function $F\left(v_{i}, v_{\text {mod }}\right)$ for each of these frequencies weighted with the corresponding line strengths. A calculation based on a large $N$ expansion of a two-dimensional $O(N)$ field theory $[36,40]$ yielded a scalar response function for the homogeneous and commensurate system of the form

$$
F\left(v, v_{\text {mod }}\right) \propto \frac{v_{\text {mod }}^{3}}{\left(v_{\text {mod }}^{2}-v^{2}\right)^{2}+4 \gamma^{2} v_{\text {mod }}^{2}} .
$$

A parametrization of the $N=2$ case of the model can be found in Refs. [138] (see also Sec. 8.2) and yields $h \gamma / U=\frac{1}{8}$. Assuming this response function at each individ- 


\section{Detection of the Higgs amplitude mode at the $2 \mathrm{~d}$ SF-Mott-insulator transition}

ual normal mode (and measuring all frequencies in units of $U / h$ ) results in the final model function

$$
F_{h}\left(v_{\text {mod }}\right)=A_{1}+A_{2} \sum_{i} S_{i} \frac{v_{\text {mod }}^{3}}{\left(v_{\text {mod }}^{2}-v_{i}^{2}\right)^{2}+4 \gamma^{2} v_{\text {mod }}^{2}},
$$

with $\gamma=\frac{1}{8}$ and fit parameters $A_{1}$ and $A_{2}$. An implicit assumption of this approach is a continuum of phase modes, which is approximately valid in our case because the frequency spacing between different phase modes is much smaller than the typical gap to the lowest amplitude mode (Sec. 9.9).

The model yields quantitative agreement with the low- to intermediate-frequency experimental data for a range of couplings (dashed black line in Fig.9.6d and Fig. 9.8), where a relativistic field theoretical treatment of this might be applicable. The good agreement suggests the intuitive interpretation that the signal consists of a sum of spectral lines, whereas each line is broadened due to the interaction of amplitude- and phase-modes.

The model, however, yields a slightly too smooth spectral onset for values of the coupling $j$ close to the critical point (see Fig.9.8). We attribute this partly to the fact that the width of the response function $\gamma$, within the large $N$ expansion, stays constant approaching the critical point. In contrast, the experiment signal, shows a reduction of the absolute width approaching the critical point (see Fig. 9.3). A further mismatch occurs when going to large $J / U$ values, where Eq. 9.1 predicts a constant width, while the experimental signal broadens (Sec. 9.5). We also mention that the response function does not fulfill the universal scaling of $F\left(v, \frac{j}{j_{c}}\right)_{u}$ described in the previous section (see Ref. [41] for a discussion).

\subsubsection{High frequencies and $2 \times 2$ cluster mean field simulation}

The heuristic model slightly underestimates the response at frequencies higher than twice the absorption edge. Part of this high-frequency response might stem from the excitation of several amplitude modes or combinations of amplitude and phase modes, which cannot be described with the Gutzwiller approximation used so far and is only partly captured in the field theoretical treatment. Therefore, we performed a dynamical simulation based on a $2 \times 2$ cluster variational wave function (Sec.9.8), which captures the excitation of multiple modes as well as inter-mode coupling, at least at high momenta. The result is compared with experimental data in Fig. 9.6d and shows good overall agreement (also compare Fig.9.9a and 9.9b near the critical point). Notably, the simulation predicts the low-frequency edge, the overall width and the absolute strength of the experimental signal without any fitting parameters. The simulation, which also accounts for fluctuations of the experimental 

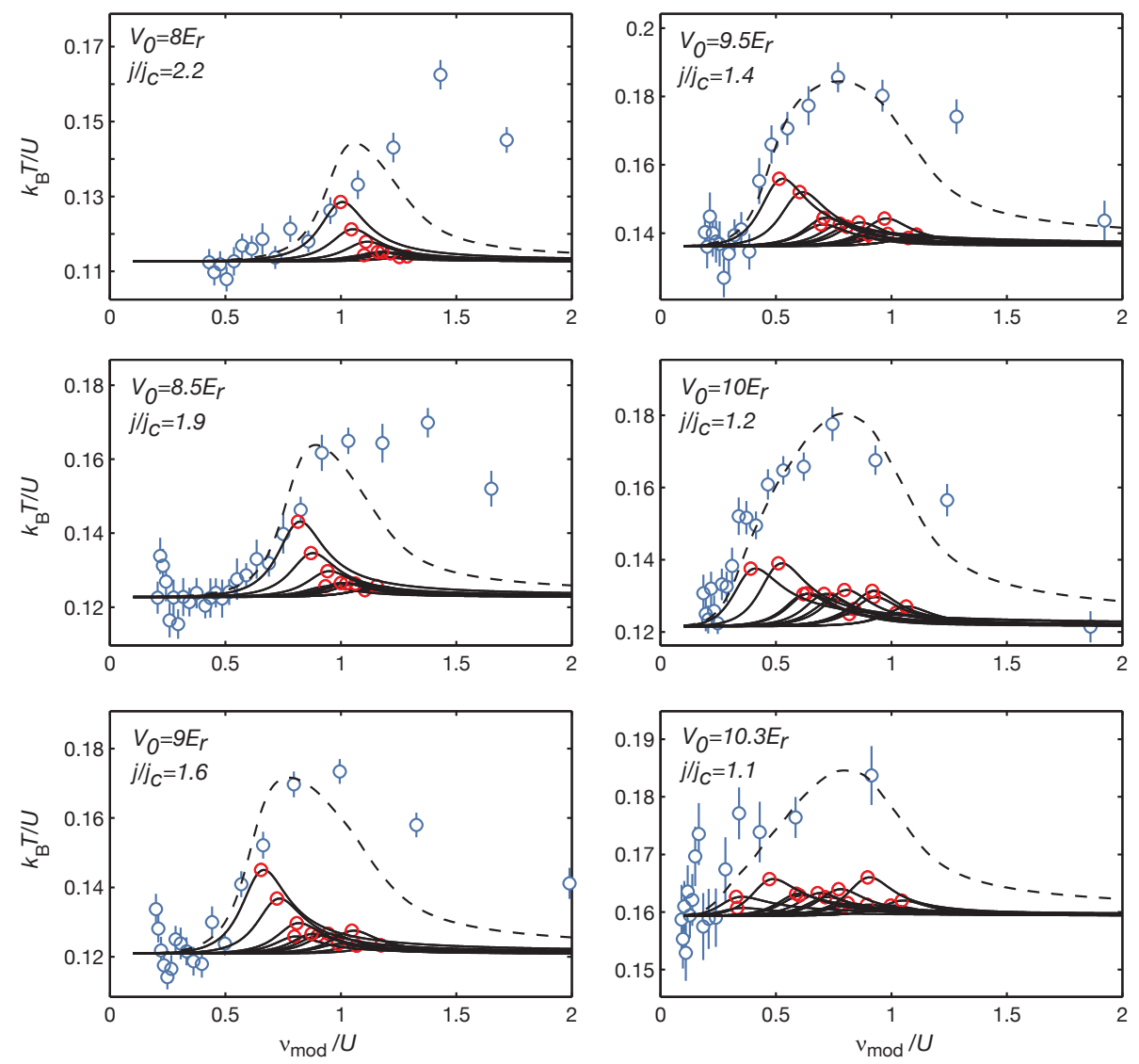

Figure 9.8.: Heuristic model. Fit of the data in the superfluid regime (blue circles) with a heuristic model (dashed line). For details concerning the model see text. The individual contributions $A_{2} S_{i} F\left(v_{i}, v_{\text {mod }}\right)$ are shown as solid lines. Red circles mark the frequency position $v_{i}$ of the corresponding normal mode.

parameters, shows a fine structure which is not observed in the experiment. This indicates that the $2 \times 2$ cluster treatment still cannot fully capture the broadening of the modes due to coupling with low-energy phase modes. 


\subsection{Vanishing of the response in the weakly interacting limit}

Our analysis, so far, has shown the existence of an amplitude mode in the BoseHubbard model close to the critical point $\left(j / j_{c} \approx 1\right)$, where the low-energy description of the system is approximately Lorentz invariant. In the weakly interacting limit $\left(j / j_{c} \gg 1\right)$, however, the low-energy description (Gross-Pitaevski theory) forbids the existence of such a mode [153] (see also Sec. 8.4). To probe the evolution of the amplitude-mode response when approaching the weakly interacting limit, we extended our measurements to higher values of the coupling $j$. The results are shown in Fig. 9.9a as a density plot, where a pronounced signal for $j / j_{c} \lesssim 3$ directly shows the softening of the mode close to the critical point. Approaching the weakly interacting limit with higher $j / j_{c}$ values, the response gradually broadens and finally disappears. Despite earlier theoretical treatments of the system in this regime [145, 154], a prediction of the disappearance of the response is still lacking. Also, results from the $2 \times 2$ cluster variational wave function approximation could only partially capture this effect (Fig. 9.9b). 


\subsection{Vanishing of the response in the weakly interacting limit}

a
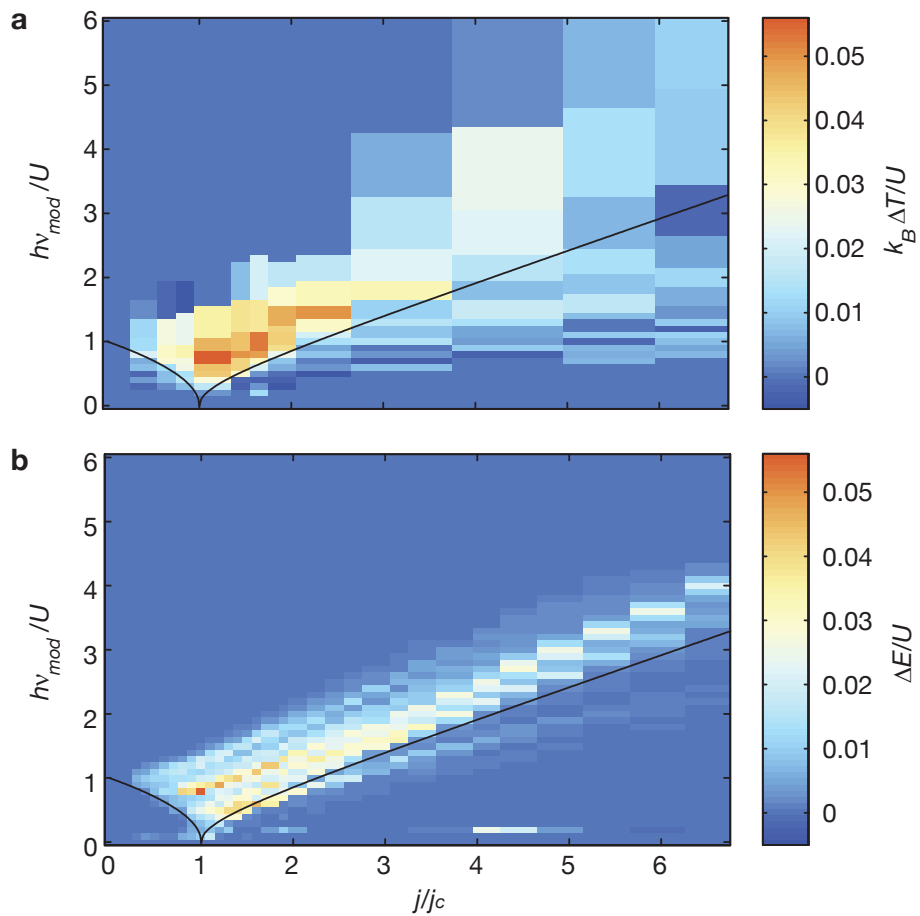

Figure 9.9.: Response from the strongly to the weakly interacting limit. a, Change in Temperature $\Delta T$ as a function of $j / j_{c}$ and the modulation frequency $v_{\text {mod }}$ in units of $U$. A pronounced feature close to $j / j_{c}=1$ directly shows the existence of the gap and its softening, which is also observed in units of $J$ (see Fig. 9.10). Approaching the weakly interacting limit (higher $j / j_{c}$ ), the response broadens and vanishes. b, Simulation using a variational $2 \times 2$ cluster wave function predicting the energy absorption per particle $\Delta E$ for the same parameter range. The simulation shows agreement with the experimental data near the critical point in both the softening of the response and the overall width of the absorption band. However, the simulation does not fully reproduce the vanishing of the response at higher $j / j_{c}$ values. A splitting in the excitation structure at $j / j_{c} \approx 3$ is visible, which might also be present in the experimental data. A low-frequency feature associated with density oscillations at the edges of the trap due to the excitation of phase-like modes is clearly seen in the simulations. This feature occurs below the lowest measured frequency in the experiment and thus is not visible in a, (except in the vicinity of the critical point, where the lowest modulation frequencies are close to this feature). Black solid lines show the mean field predictions as plotted in Fig. 9.2a. 


\subsection{Softening with respect to $J$}

Up to now we scaled all frequency axes with $U$. One might wonder if the observed softening also holds with respect to J. In Fig. 9.10, we show all data sets as a density plot with the frequency axis scaled with $J$. The theory prediction for the superfluid (black line for $j / j_{c}>1$ ) becomes approximately linear in $J$ for large $j / j_{c}$. This indicates that a softening with respect to $J$ is not expected for $j / j_{c} \gtrsim 3$. However, the strongest experimental response occurs for $j / j_{c} \lesssim 3$ and the softening of the spectral onset is clearly visible in this range. This demonstrates that we observe softening of the response close to the critical point with respect to $U$ and to $J$.

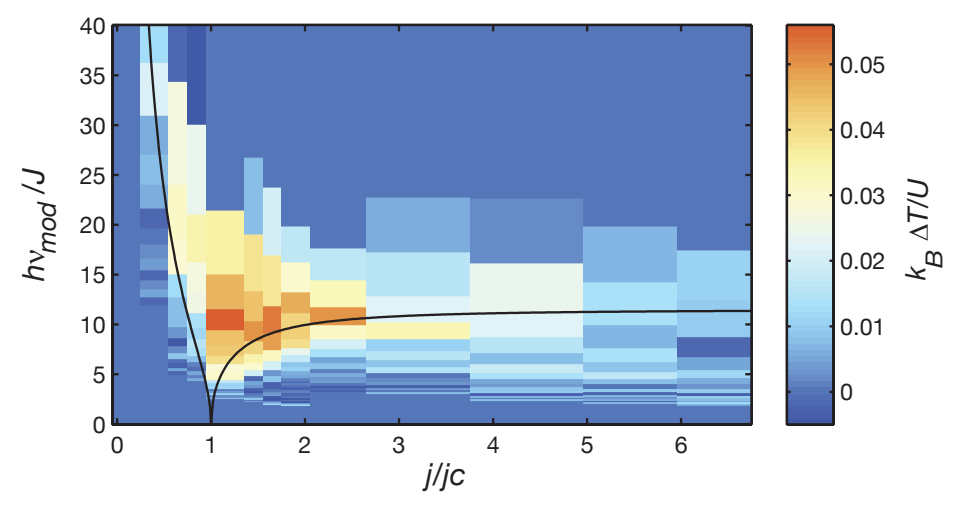

Figure 9.10.: Response in units of $J$. Figure shows the same data and theory curves as Fig. 9.9a, except for the $y$-axis being scaled with the tunnel coupling $J$ instead of the on-site interaction $U$.

\subsection{Discussion of finite-temperature effects}

Based on the fact that the critical temperature for superfluidity tends to be reduced close to the critical point (see Fig. 2.3), one might wonder whether our system still shows properties of a superfluid for values of $j$ close to the critical point.

In general, we would like to stress that the initial temperature of the sample ( $T / U \approx 0.10$ ) has to be viewed as a conservative upper bound. A number of processes (e.g., the final lattice ramp, the hold time during modulation and losses during the imaging) tend to increase the measured temperature compared to the actual initial temperature of the sample at the beginning of the modulation time. We estimate the actual initial temperature to be in a range from $T / U=0.07$ to $T / U=0.1$ based on the experiments described in Part I and II.

For these temperatures and for a system of $32 \times 32$ sites QMC simulations have been performed [155], which show that the system contains a finite superfluid fraction even very close to the critical point (for couplings $J / U$ down to $\approx 0.06$ ). Also, recent 
QMC simulations for a system of $14 \times 14$ sites at $J / U=0.07\left(j / j_{c} \approx 1.2\right)$ show that for typical experimental temperatures the superfluid fraction remains higher than $60 \%$ of the maximum superfluid fraction achievable at zero temperature [156]. For all data points further away from the critical point $\left(j / j_{c}>1.2\right)$, one expects this value to be even significantly higher. In summary, both studies indicate that our system shows properties of a strongly interacting superfluid even for the data points closest to the critical point.

We also note recent predictions for the response to lattice modulation based on QMC and an analytic continuation scheme [41]. This study confirms our approach to use the onset of spectral response (of a trapped system) to extract information on the Higgs mode properties of the homogeneous system. Furthermore, the simulations suggest that the onset of spectral density is a robust feature which persists at finite realistic temperatures.

\subsection{Gutzwiller calculation and $2 \times 2$ cluster wave functions}

In the following, we describe the Gutzwiller calculation and the $2 \times 2$ cluster mean field simulation. The author of this thesis would like to stress that these calculations have been performed by David Pekker (see Ref. [157] for author details). The methods are described here for the sake of completeness and for the discussion of the differences between amplitude and phase modes in the following section.

\section{Gutzwiller calculation of the Eigenmodes in a trap}

To perform the eigenmode analysis, we used the Gutzwiller trial wave function $[49,147]$

$$
\left|\Psi_{1 \times 1}\right\rangle=e^{i \phi} \prod_{i}\left(\alpha_{i}(t)|0\rangle_{i}+\sqrt{1-\left|\alpha_{i}(t)\right|^{2}-\left|\gamma_{i}(t)\right|^{2}}|1\rangle_{i}+\gamma_{i}(t)|2\rangle_{i}\right),
$$

where $\alpha_{i}(t)$ and $\gamma_{i}(t)$ are variational parameters, $|n\rangle_{i}$ corresponds to a state with $n$ bosons on site $i$, and $\phi$ is an overall phase. First, we obtained the stationary solution $\left|\Psi_{1 \times 1}^{0}\right\rangle$ (corresponding to $\left\{\alpha_{i}^{0}, \gamma_{i}^{0}\right\}$ ) by minimizing $\left\langle\Psi_{1 \times 1}^{0}\left|H_{\mathrm{BH}}\right| \Psi_{1 \times 1}^{0}\right\rangle$ in the entire trap. Next, we linearized the equations of motion, which were obtained by minimizing the effective action $\left\langle\Psi_{1 \times 1}\left|i \partial_{t}-H_{\mathrm{BH}}\right| \Psi_{1 \times 1}\right\rangle$ around the stationary solution. The resulting eigenvalue problem was solved by a Bogoliubov transformation $M_{k, i r}$ that relates Bogoliubov creation $f_{k}^{\dagger}$ (and annihilation $f_{k}$ ) operators to the small fluctuations $\delta \alpha_{i}$ and $\delta \gamma_{i}$ around the stationary solution

$$
f_{k}^{\dagger}=\sum_{i}\left(M_{k, i 1} \delta \alpha_{i}+M_{k, i 2} \delta \alpha_{i}^{*}+M_{k, i 3} \delta \gamma_{i}+M_{k, i 4} \delta \gamma_{i}^{*}\right),
$$




\section{Detection of the Higgs amplitude mode at the $2 \mathrm{~d}$ SF-Mott-insulator transition}

where $k$ is the eigenmode index. We can identify the modes as amplitude-like or phase-like using a measure of 'amplitudeness'

$$
A=\sum_{i} M_{k, i 1} M_{k, i 3}
$$

which is positive for amplitude-like modes and negative for phase-like modes (see following section).

To describe lattice modulation spectroscopy, we separated the Bose-Hubbard Hamiltonian into a time independent part that describes the system with no modulation and a time dependent part that describes the lattice modulation: $H_{\mathrm{BH}}=H_{0}+\sin (\omega t) H^{\prime}$. The rate of excitation of the $k$-th amplitude or phase mode is given by Fermi's golden rule: $\Gamma(\omega)=\delta\left(\omega-\omega_{k}\right)\left|\left\langle\Psi_{1 \times 1}^{0} f_{k}\left|H^{\prime}\right| \Psi_{1 \times 1}^{0}\right\rangle\right|^{2}$. The line strengths plotted in Fig. 9.6a are proportional to $S_{i}=\left|\left\langle\Psi_{1 \times 1}^{0} f_{k}\left|H^{\prime}\right| \Psi_{1 \times 1}^{0}\right\rangle\right|^{2}$. The line strengths decrease with increasing frequency, because higher energy modes show short wavelength spatial variations and do not efficiently couple to lattice modulation.

\section{Dynamical evolution: $2 \times 2$ cluster wave functions.}

We performed a study of the dynamical evolution of the system using $2 \times 2$ cluster variational wave functions

$$
\left|\Psi_{2 \times 2}\right\rangle=\prod_{i}\left[a_{i}(t)\left|\begin{array}{ll}
0 & 0 \\
0 & 0
\end{array}\right\rangle+b_{i}(t)\left|\begin{array}{ll}
1 & 0 \\
0 & 0
\end{array}\right\rangle+c_{i}(t)\left|\begin{array}{ll}
0 & 1 \\
0 & 0
\end{array}\right\rangle+\ldots\right],
$$

where $a_{i}(t), b_{i}(t), c_{i}(t), \ldots$ are the variational parameters. We restrict the maximum occupation number per site to two. To initialize the dynamics, we obtained the initial trial wave function, corresponding to the state of the system before modulation spectroscopy begins, by minimizing $\left\langle\Psi_{2 \times 2}\left|H_{\mathrm{BH}}\right| \Psi_{2 \times 2}\right\rangle$. Next, we dynamically evolved the trial wave function during the modulation drive, the hold time and the ramp to the atomic limit. Finally, we measured the total energy absorption per particle $\Delta E$ of the resulting state (in units of the on-site interaction $U$ in the atomic limit).

\subsection{Distinguishing phase and amplitude modes}

We verify that eigenmodes show amplitude-like or phase-like character by applying the amplitude measure (Eq.9.5). For each eigenmode, we compute the amplitude measure and plot it as a function of the eigenfrequency (Fig. 9.11a). The result shows two branches: a branch at lower frequencies with negative measure that corresponds to phase-like modes, and a gapped branch at higher frequencies with positive measure that corresponds to amplitude-like modes.

Importantly, lattice modulation couples only to a few modes of the full spectrum (see Fig. 9.11b). Most modes have strictly vanishing coupling strengths, because 


\subsection{Distinguishing phase and amplitude modes}
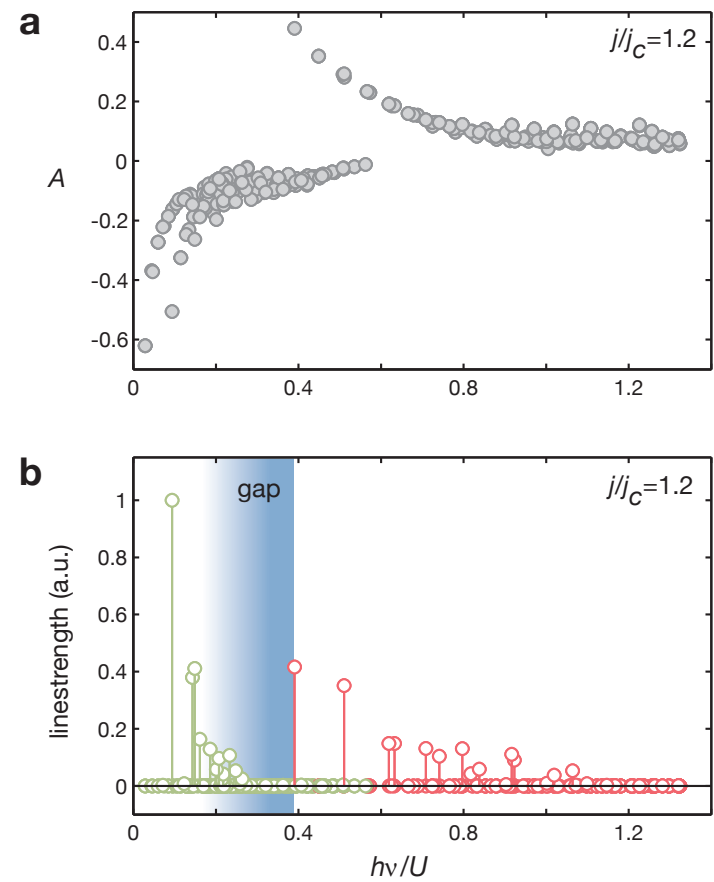

Figure 9.11.: Amplitude measure and line strength. a, Amplitude measure as a function of eigenfrequency for a trapped system at $j / j_{c}=1.2$. Amplitude-like (phase-like) modes have positive (negative) amplitude measure. $\mathbf{b}$, Line strength for the same mode spectrum. Amplitude-like (phase-like) modes are shown in red (green). A large number of modes have strictly zero coupling strength due to their spatial symmetry. From the modes, which are allowed by symmetry, only the ones with long wavelength spatial variations (low-energy) couple significantly to lattice modulation. This leads to an effective gap between the amplitudelike and phase-like response (blue shading). The effective gap increases with the distance to the critical point (Fig. 9.12).

their spatial symmetry prevents an excitation with lattice modulation where both axes are driven in phase and with the same amplitude. From the remaining modes, only the ones with the lowest frequencies of the respective branch show a significant coupling. At higher frequencies, the modes are dominated by short wavelength spatial variations avoiding an efficient coupling to uniform lattice modulation.

This results in an effective gap between the response from phase and amplitude-like modes (Fig. 9.11b and Fig. 9.12). In the experiment, both responses are therefore expected to be well separated. In the experimental spectra shown in Fig. 9.4, a response from phase-like modes might be visible for the lowest experimental frequencies (e.g., for $V_{0}=9 E_{r}$ at $v_{\mathrm{mod}} \approx 80 \mathrm{~Hz}$ or for $V_{0}=10.3 E_{r}$ at $v_{\mathrm{mod}} \approx 80 \mathrm{~Hz}$ ). In all cases, these features are separated from the main peak and do not alter the analysis of the main response, except for the low-frequency scaling in Fig. 9.7, where the lowest frequency 


\section{Detection of the Higgs amplitude mode at the $2 \mathrm{~d}$ SF-Mott-insulator transition}

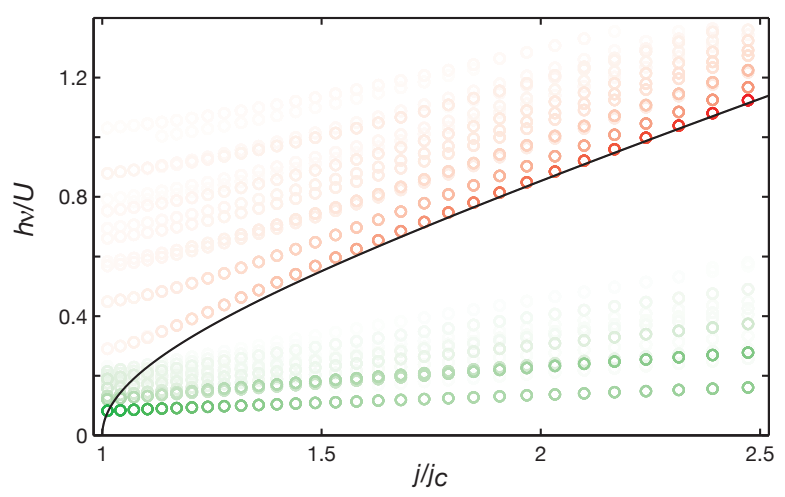

Figure 9.12.: Normal mode spectrum as a function of $j / j_{c}$. Each circle corresponds to an eigenmode with the intensity of the color being proportional to the line strength. Amplitudelike (phase-like) modes are shown in red (green). The response from phase-like modes is only visible at low frequencies and separated from the amplitude-like response by a gap. The experimental response in the main amplitude-mode peak is therefore expected to be unaltered by the presence of phase-like modes.

data points were excluded. A response from phase-like modes is also visible for the cluster wave function data shown in Fig.9.9b as a sharp feature at very low frequencies, which is well separated from the main response.

The same generic behaviour of the amplitudeness measure is also seen in a homogeneous system (Fig.9.13). Two branches with negative and positive measure are visible, corresponding to phase-like and amplitude-like excitations. The amplitudeness measure is also related to the time evolution of the respective modes given by the trajectories of the order parameter $\Psi$ in the complex plane. For a given mode, the trajectory is an ellipse around the equilibrium value of $\Psi$ (Fig. 9.13c) and the full ellipse is passed in a time $1 / v$, where $v$ is the excitation frequency of the mode. To characterize the ellipses, we introduce the flatness parameter $f=(a-b) /(a+b)$, where $a$ and $b$ are the axes of the ellipse as defined in Fig. 9.13c. The flatness parameter $f$ (Fig. 9.13b) also shows two branches corresponding to phase- and amplitude-like modes, with a similar behaviour as the amplitudeness measure. In the respective branches, the modes with the lowest energies correspond to low-momentum modes. For these modes, the trajectories are very flat ellipses (large $|f|$ ) with their major axis perpendicular ( $f<0$, phase-like) or parallel $(f>0$, amplitude-like) to the direction of the mean order parameter. With increasing momentum the modes become more circular, with a flatness parameter close to zero.

Notice that this behavior is different to the one observed in the $O(2)$ theory (Fig. 8.3). The fact that in the $O(2)$ theory both modes are purely in the amplitude and phase direction for all momenta can be traced back to the strict particle-hole symmetry. With 


\subsection{Distinguishing phase and amplitude modes}

a
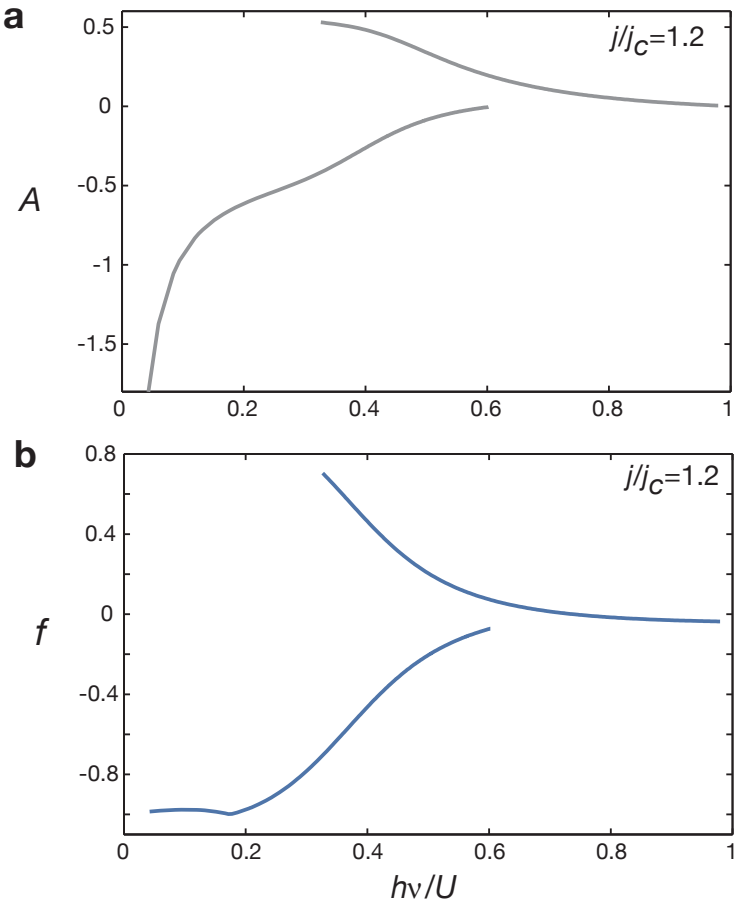

C

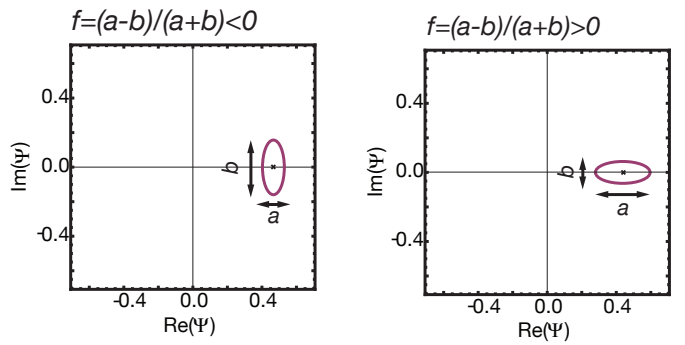

Figure 9.13.: Phase- and amplitude-like modes for a homogeneous system. a, Amplitudeness measure $A$ for a homogeneous system at $j / j_{c}=1.2$ as a function of frequency $v$. Two branches with decreasing magnitude of the amplitudeness measure are visible. Negative and positive values correspond to phase- and amplitude-like modes, respectively. The calculation was done in the Gutzwiller approximation (see Sec. 9.8) for a square system with a length of 40 sites applying periodic boundary conditions. b, Flatness $f=(a-b) /(a+b)$ of the trajectories of the order parameter in the complex plane as a function of frequency $v$. Again, two branches corresponding to phase-like (starting from $f<0$ ) and amplitude-like (starting from $f>0$ ) modes are visible. c, Illustration of the flatness parameter $f$ showing typical trajectories for the order parameter $\Psi$ in the complex plane. For both panels the mean value of $\Psi$ is 0.47 along the real axis (small cross). For $f<0$ (left panel), the trajectory is an ellipse around the mean value, with its major axis perpendicular to the real axis (i.e., the direction of the order parameter). For $f>0$ (right panel), the major axis is parallel to the real axis. 
this we mean that states with an extra and a missing particle appear with the same quantum mechanical weight (Eq. 8.8). In contrast, the Gutzwiller approximation allows for different weights (Eq.9.3), which leads to the finite ellipticity in Fig. 9.13 for high momenta. The field-theoretical description is therefore only reasonable at low momenta.

The main difference between the homogeneous and the trapped system is the possibility to excite certain modes in the spectrum with lattice modulation. In an infinitely large homogeneous system, only modes with zero momentum can be excited with lattice modulation (the lowest energy mode of the respective branches). In a large enough trapped system, this selection rule is still approximately valid in the sense that only a few modes with the lowest energies of the respective branches couple significantly (see Fig. 9.11b). The spatial pattern of these modes show only long-wavelength variations (equivalent to low-momentum in a trapped system). 


\subsection{Summary and conclusion}

In conclusion, we could identify and study long-wavelength Higgs modes in a neutral two-dimensional superfluid close to the quantum phase transition to a Mottinsulating state. This was enabled by recent advances in the high-resolution imaging of single atoms in optical lattices (Part I), leading to a new sensitive method for the spectroscopy of ultracold quantum gases based on a combination of lattice modulation and in-situ temperature measurement. The obtained spectra show softening at the quantum phase transition and are consistent with the generic $v^{3}$ lowfrequency scaling for a rotationally invariant coupling to the order parameter in a two-dimensional strongly interacting superfluid. Furthermore, our results challenge the development of a quantitative theory valid between the strongly and the weakly interacting regimes capable of predicting the observed disappearance of the response. Our data also call for a first-principle treatment of the discrete nature of Higgs modes in a confined system. 
9. Detection of the Higgs amplitude mode at the 2d SF-Mott-insulator transition 


\section{Outlook}

The demonstrated single-atom- and single-site-resolved detection of an ultracold bosonic quantum gas in an optical lattice and its application for the measurement of correlation and response functions opens up many new possibilities for future studies.

In particular, the ability to measure higher-order correlation functions offers a novel experimental way of characterizing quantum many-body systems. In the long term, however, one wishes to develop schemes that are not restricted to density-density type correlation functions (see Sec.3.5). A major experimental step would therefore be the single-atom- and single-site-resolved detection of the single-particle density matrix $\left\langle\hat{b}_{i}^{+} \hat{b}_{j}\right\rangle$, where $\hat{b}_{i}^{+}\left(\hat{b}_{j}\right)$ are creation (annihilation) operators, in order to gather information about the phase coherence in the system. This might be achievable with an in situ detection of interfering one- or two-dimensional systems [81, 158, 159]. Based on this, a long-term goal would be the measurement of correlators involving higher orders of creation and annihilation operators that do not resemble density terms. One particularly interesting application is the investigation of entanglement in many-body systems [44]. A recent proposal showed that this should already be possible using a combination of existing experimental techniques [45].

In a recent work [31], we used the single-atom-resolved measurement of correlations in ordered structures formed by Rydberg excitations in a Mott insulator. In this experiment, the motional degree of the atoms was frozen. Using off-resonant Rydberg dressing, long-range interactions in a free-to-move quantum gas could be engineered $[160,161]$. This could be used to realize an extended Hubbard model with longer-range interactions that features a Haldane insulating phase [99, 100]. This phase shows a non-local order that is similar to the hidden antiferromagnetic order described in Sec. 6.1. Furthermore, Rydberg dressing might also to be a promising route to realize a supersolid state [162-164].

In addition to the new imaging technique, the possibility to manipulate single particles in an optical lattice [27] opens up new ways of probing quantum manybody systems. Recently, we used this technique for the controlled preparation of a single impurity in a strongly interacting one-dimensional system and recorded its subsequent motion with single-site resolution [28]. The same scheme can also be used to prepare a pair of impurities. Effective attractive interactions between the impurities, induced by the strongly-interacting background, might be detectable in spatial correlations of the impurity positions [165]. Furthermore, this setup has 
the potential for the position- and time-resolved detection of spin-charge separation [166-168].

The same technique can also be used for a spatially resolved control of the Hamiltonian parameters. One immediate application is the flexible creation of potential landscapes $[169,170]$. This has a range of different applications, such as the study of transport through mesoscopic channels [171] or the study of persistent flow in toroidal traps [172]. Investigations of Hubbard-type models could also benefit from such an approach. For example, engineering the local chemical potential could be used to create a box-shaped potential. In the long run, one could even imagine achieving local control over the nearest neighbor coupling or the on-site interaction to realize a situation where $J / U$ varies spatially. With this, one could observe a cut in the Bose-Hubbard model along the $J / U$ direction, in contrast to the usual situation of a cut in the $\mu$ direction. It would be interesting to study proximity effects at the tip of the lobe transition similar to the ones described in Ref. [173] for the generic transition. In a second step, one could additionally implement time and space-dependent variations of $J / U$. With this, momentumresolved lattice modulation spectroscopy might be feasible. To achieve this, one could vary $J / U$ in a wave-like fashion to probe excitations with a certain wavelength.

Given these possibilities, it is a natural step to implement single-site- and singleatom-resolved imaging and manipulation techniques for stongly interacting fermions in optical lattices [4-6]. In particular, a possible antiferromagnetic ordering at low temperatures should be directly detectable in a single-site-resolved correlation function. Currently, major experimental efforts are being undertaken to achieve this goal, for example, using ${ }^{40} \mathrm{~K}$ or ${ }^{6} \mathrm{Li}$. One of the experimental challenges is achieving sufficient cooling during the imaging phase, because standard sub-Doppler cooling for these elements is not as efficient as for ${ }^{87} \mathrm{Rb}$. However, recent advances in this respect might help to overcome this problem (see Ref. [174] and references therein).

Finally, the regular, almost defect-free arrangement of atoms in the deep Mottinsulating limit combined with single-site addressing might be a promising setup for quantum computing. As a next step, one would like to implement single-siteresolved, arbitrary qubit-rotations, in addition to $\pi$-pulses [74]. Two-qubit gates could be based on Rydberg interactions, as already demonstrated in optical tweezers $[175,176]$, or on local collisional interactions [177]. Another route might be one-way quantum computing $[178,179]$ based on a resource state that can be created with a global entanglement operation [180,181]. For this, the single-site-resolved readout of the atomic spin state without detecting the remainder of the system is a prerequisite, which is a technique that is still to be demonstrated.

In summary, the new high-resolution and single-particle-resolving technique holds the potential to have a major impact on the investigation of correlated many-body systems and applications of such systems. 
10. Outlook 


\section{Part IV.}

\section{Appendix}





\section{A. Experimental details}

In this appendix, we give a more detailed description of the experimental setup. A detailed description has already been given in Ref. [74] but several changes have been made. The data presented in Part I have been taken with the setup as described in Ref. [74], while the changes apply to Parts II and III. These modifications mainly concern the preparation of the $2 \mathrm{~d}$ degenerate cloud. In the following, we focus on this preparation procedure.

\section{A.1. Preparation of the two-dimensional quantum gas}

\section{A.1.1. Overview}

We first give an overview of the complete preparation sequence and give details for the individual steps afterwards. The vacuum apparatus consists of two steel chambers that are connected with a differential pumping section. The first chamber is used as a $2 \mathrm{~d}$ magneto-optical trap $(2 \mathrm{~d}-\mathrm{MOT})$ that serves as a source for a $3 \mathrm{~d}$ magnetooptical trap (3d-MOT) in the second chamber (Fig. A.1). The 3d-MOT was loaded from the $2 \mathrm{~d}$-MOT for about $2 \mathrm{~s}$, yielding a total atom number of about $10^{9}$. The atoms were then transferred into a quadrupole magnetic trap. This was followed by a forced evaporation using microwave transition from the trapped state $\left|F=1, m_{F}=-1\right\rangle$ to the anti-trapped state $\left|F=2, m_{F}=-2\right\rangle$. If not stated differently, this notation refers to the hyperfine ground state manifold $5^{2} S_{1 / 2}$ of ${ }^{87} \mathrm{Rb}$ [75]. We stopped the evaporation just before Majorana losses set in, ending up with about $10^{8}$ atoms at $20 \mu \mathrm{K}$.

Afterwards, we transferred the atoms into a single-beam optical dipole trap. We used this trap to transport the cloud in front of the high-resolution imaging system by moving the focus of the beam using a translation stage. After the transport, the atoms were loaded into a crossed dipole trap, which consisted of the horizontal lattice beams whose retro-reflections were blocked with mechanical shutters. We evaporatively cooled the atoms in this trap by changing its depth from $20 \mu \mathrm{K}$ to $10 \mu \mathrm{K}$ within $1 \mathrm{~s}$, before transferring the atoms into a vertical standing wave of which we typically populate 40 antinodes. To extract a single two-dimensional system, we used a positiondependent microwave transfer in a magnetic field gradient of $\partial B / \partial z=45 \mathrm{G} / \mathrm{cm}, 1.9$ times larger compared to Ref. [74]. After a transfer of all atoms from $\left|F=1, m_{F}=-1\right\rangle$ to $\left|F=2, m_{F}=-2\right\rangle$ with a broad microwave sweep, the atoms from one antinode of the standing wave were transferred back to $\left|F=1, m_{F}=-1\right\rangle$ using a HS1-pulse [182] of $5 \mathrm{~ms}$ duration and a sweep width of $3.5 \mathrm{kHz}$. The remaining atoms in state 


\section{A. Experimental details}

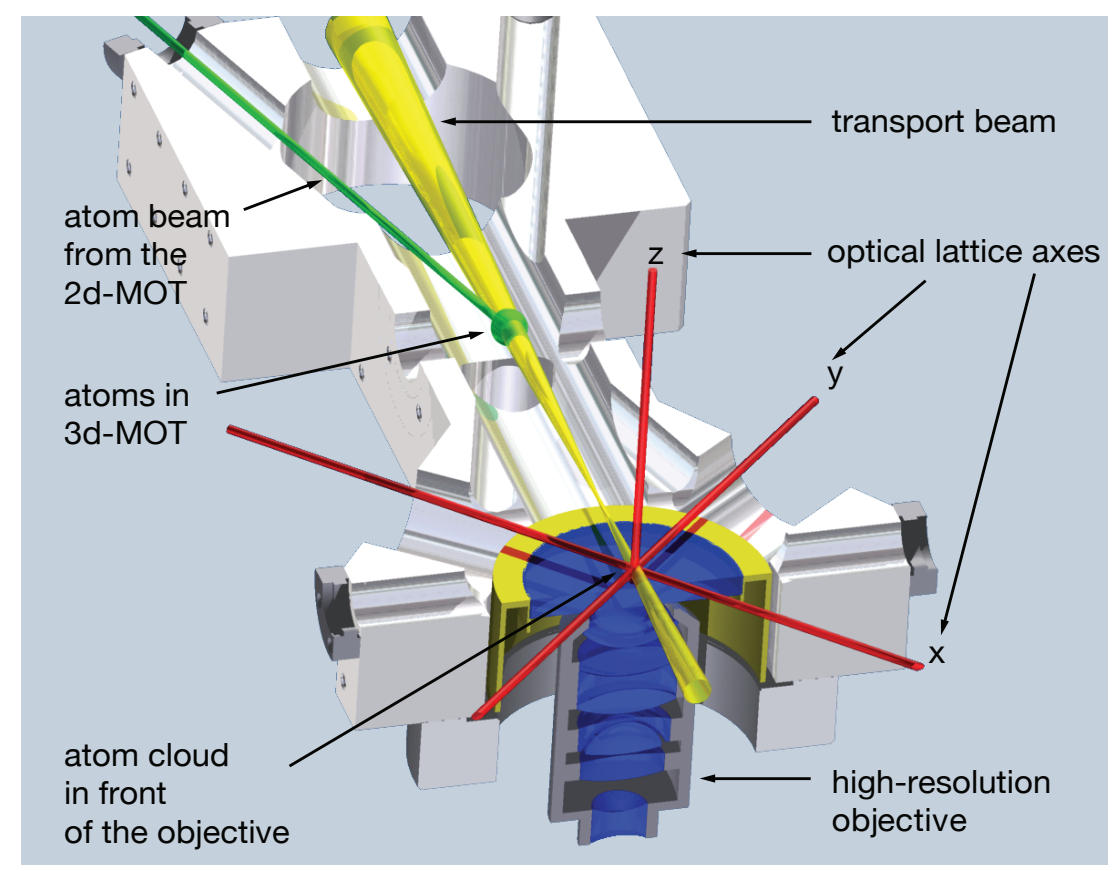

Figure A.1.: Overview of the experimental vacuum chamber. Cut through the main vacuum chamber and the high-resolution objective. Additionally, the atom beam from the 2d-MOT loading the 3d-MOT is shown in green. The single-beam transport trap is drawn in yellow and the optical lattice axes are shown in red. The $z$-optical lattice axis is in vertical direction.

$F=2$ were removed from the trap by a laser pulse resonant with the $5 S_{1 / 2}, F=2$ to $5 P_{3 / 2}, F=3$ transition.

For the final evaporation, we added a focused laser beam of wavelength $850 \mathrm{~nm}$ shone in through the imaging system (dimple trap), with a beam waist of $\sim 10 \mu \mathrm{m}$ at the position of the atoms. The intensity of the beam is adjusted in such a way that a small number of atoms is lost due to three-body recombination, resulting in a stabilization of the atom number. In this configuration, we evaporatively cooled the atoms by tilting the trap horizontally with a magnetic field gradient of $11.25 \mathrm{G} / \mathrm{cm}$ and by reducing the power of the $850 \mathrm{~nm}$ beam. We adjusted the atom number by changing the end point of this evaporation and achieved an atom number stability better than $20 \%$.

\section{A.1.2. MOT and magnetic evaporation}

\section{2d-, 3d MOT and vacuum system}

The setup of the $2 \mathrm{~d}$ MOT is described in detail in Ref. [183]. We changed this setup by adding a retro-beam to form a $2 d^{+}$configuration [184]. The differential pumping section between the $2 \mathrm{~d}-\mathrm{MOT}$ and the main chamber has an inner diameter of $1.6 \mathrm{~mm}$ 


\section{A.1. Preparation of the two-dimensional quantum gas}

and a length of $51 \mathrm{~mm}$. An additional bellow allowed us to move the 2d-MOT setup with respect to the main chamber in order to optimize the loading of the 3d-MOT. The pressure in the $2 \mathrm{~d}$-MOT was $4 \cdot 10^{-7} \mathrm{mbar}$, whereas we reached the $10^{-11} \mathrm{mbar}$ range in the main chamber. The pumps for the main chamber were an Ion-getter pump (Varian, VacIon Plus 55) and a Titanium-sublimation pump (VG Scienta, SBST22).

The laser setup for the 3d-MOT is described in Ref. [74]. All laser beams are coupled to optical fibers and can be blocked by mechanical shutters. Acousto-optic modulators (AOM) allow for fast changes in the intensity. The 3d-MOT is a six-beam MOT and we used a single $1: 5$ telescope to widen the beams to a diameter of $\approx 25 \mathrm{~mm}$ instead of separate telescopes for each axis. The re-pumping light is overlapped with the cooling light before this telescope and the light is divided to the six beams after the telescope using polarizing beam splitters.

During the loading phase we used a magnetic gradient of $23 \mathrm{G} / \mathrm{cm}$ and a $-15 \mathrm{MHz}$ detuning for the cooling light of the 3d-MOT.

\section{Loading to the magnetic trap and coil details}

For the loading to the magnetic trap, we first switched off the cooling light and the push-beam of the 2d-MOT. Afterwards, we employed a compressed-MOT for $100 \mathrm{~ms}$ by increasing the cooling-light detuning to $-26 \mathrm{MHz}$ and reducing the gradient to $11.5 \mathrm{G} / \mathrm{cm}$. This was followed by a $14 \mathrm{~ms}$ molasses phase, during which we detuned to $-125 \mathrm{MHz}$ and switched off the magnetic gradient completely. Then, we switched on the magnetic gradient to $50 \mathrm{G} / \mathrm{cm}$ in $300 \mu \mathrm{s}$ and increased it to the final value of $184 \mathrm{G} / \mathrm{cm}$ in $25 \mathrm{~ms}$.

We used no additional optical pumping step and reached a total transfer efficiency of more than $50 \%$ to the magnetic trap, where we trapped the atoms in the state $\left|F=1, m_{F}=-1\right\rangle$.

We used the same pair of coils, in an anti-Helmholtz configuration, for creating the $3 \mathrm{~d}-\mathrm{MOT}$ and the magnetic trap. The pair of coils is aligned in the horizontal direction, perpendicular to the transport beam. Each coil has 119 windings and is placed in a water-cooled housing. The atoms had a distance of about $4 \mathrm{~cm}$ to the coils. In this configuration, the pair of coils yielded $2 \cdot 2.3 \mathrm{G} /(\mathrm{cm} \cdot \mathrm{A})$. As a current supply, we used a Delta Elektronika Power Supply, SM 70-90.

\section{Evaporation in the magnetic trap}

We employed forced evaporative cooling by a microwave (MW) transfer from $\left|F=1, m_{F}=-1\right\rangle$ to the anti-trapped state $\left|F=2, m_{F}=-2\right\rangle$. We started with a detuning of $-160 \mathrm{MHz}$ from the bare resonance and ended with a detuning of 


\section{A. Experimental details}

$-10 \mathrm{MHz}$. The total duration of this $\mathrm{MW}$-evaporation was $12 \mathrm{~s}$ using a linear ramp for the MW frequency.

The final atom number after evaporation was about $10^{8}$ at a temperature of about $20 \mu \mathrm{K}$. We used no additional plug laser and stopped the evaporation at the onset of Majorana losses.

\section{A.1.3. Transport and loading to vertical lattice}

\section{Transport}

For the transport, we used a single-beam optical dipole trap with wavelength $1064 \mathrm{~nm}$ and beam waist $w_{0}=40 \mu \mathrm{m}$. The maximum trap depth was $100 \mu \mathrm{K}$. As a laser, we used a IPG photonics Ytterbium fiber laser with a bandwidth of $5 \mathrm{~nm}$. The laser had a maximum output power of $50 \mathrm{~W}$, but we operated it at only $7 \mathrm{~W}$. The laser was sent through an optical fiber (Crystal Fibre, LMA-PZ-800 with connector SMA 905) and the intensity after the fiber was stabilized using an AOM (Crystal Technology, CTI 3080-198) before the fiber.

For the transfer to the transport beam, we increased the intensity of the transport laser beam to its maximum value linearly in $250 \mathrm{~ms}$. Starting at the same time, we decreased the magnetic gradient to $10 \mathrm{G} / \mathrm{cm}$ linearly in $1.3 \mathrm{~s}$. Following this, we switched off the gradient within $250 \mathrm{~ms}$. We transferred about $10^{7}$ atoms to the transport beam.

The beam path of the transport beam after the fiber is shown in Fig. A.2. For the transport, we used a linear-motor stage (Newport XMS160). The total transport duration was $2.5 \mathrm{~s}$. The motional profile of the stage during the transport was s-shaped [185] with a maximal velocity of $300 \mathrm{~mm} / \mathrm{s}$ and a maximal acceleration of $2.5 \mathrm{~m} / \mathrm{s}^{2}$. The total transport distance was $13 \mathrm{~cm}$.

\section{Crossed dipole trap and loading to vertical lattice}

We created a crossed dipole trap using the lattice beams in $x$ - and $y$-directions with their retro-reflections blocked by mechanical shutters (beam waist $w_{0}=70 \mu \mathrm{m}$, laser wavelength $1064 \mathrm{~nm}$ ). See Sec. A.2 for details concerning the optical lattice setup. For the transfer from the transport beam to the crossed dipole trap, we increased the depth of the crossed trap to $20 \mu \mathrm{K}$ linearly within $200 \mathrm{~ms}$. Afterwards, we decreased the depth of the transport beam from its full value to zero linearly in $50 \mathrm{~ms}$.

We employed forced evaporation in the crossed dipole trap by exponentially decreasing the trap depth to $10 \mu \mathrm{K}$ in $1 \mathrm{~s}$.

Then, we exponentially increased the depth of the vertical optical lattice in $z$-direction within $200 \mathrm{~ms}$ to $40 E_{r}$. Afterwards, we decreased the depth of the crossed dipole trap to zero within $200 \mathrm{~ms}$. 


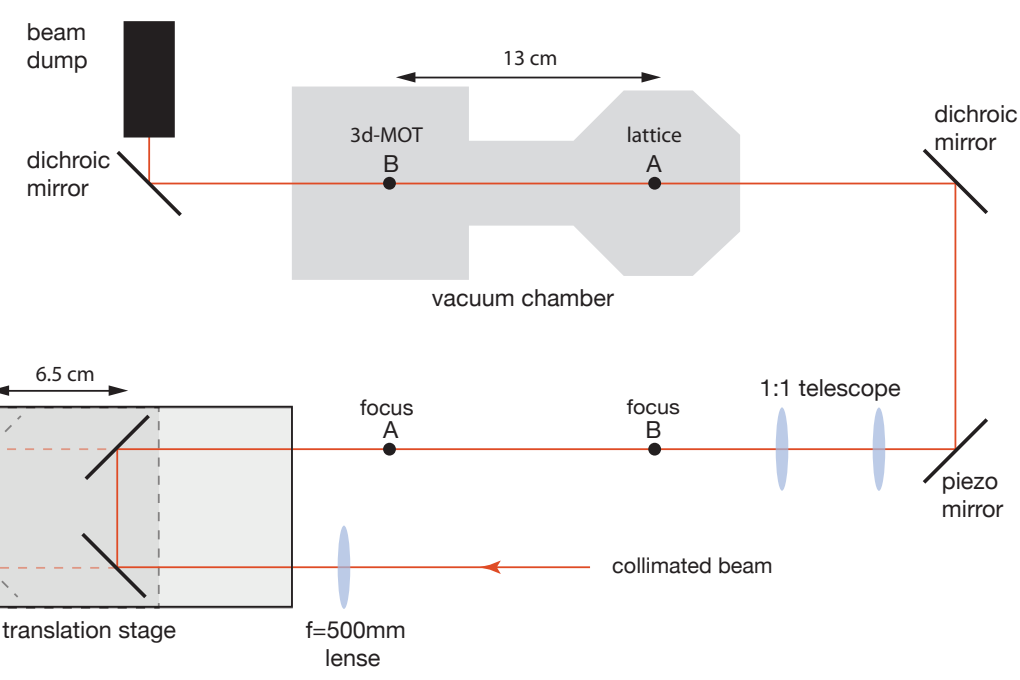

Figure A.2.: Transport setup. Sketch of the beam path for the transport beam. The collimated beam is focused using a lens with focal length $f=500 \mathrm{~mm}$. The focus position is moved by moving two mirrors on a translation stage. The two focus positions are labeled by A and B. Note that the focus position moves by twice the distance of the translation stage. The focus is then imaged onto the atoms using a 1:1 telescope (with $f=300 \mathrm{~mm}$ lenses). The beam position orthogonal to the propagation direction can be fine-tuned using a piezo mirror (Thorlabs, KC1-PZ/M). We changed this orthogonal position with the piezo mirror during the transport, which allowed for an independent adjustment at both focus positions. The beam is overlapped with a 3d-MOT axis using a dichroic mirror before the chamber and separated again using another dichroic mirror after the chamber. Several nonessential elements have been left out and the drawing is not to scale.

We could then unblock the retro-reflections in $x$ - and $y$ - direction, because both beams were switched off and the atoms were trapped only by the vertical lattice axis. Typically we populated 40 antinodes of the vertical optical lattice.

\section{A.1.4. Preparation of a single $2 \mathrm{~d}$ system and final evaporation}

\section{Preparation of a single 2d system}

For the preparation of a single $2 \mathrm{~d}$ system, we employed a technique similar to magnetic resonance imaging.

We created a magnetic gradient in z-direction using a single coil, which is displaced from the atoms by $30 \mathrm{~mm}$ in vertical direction (Fig. A.3). We refer to this coil as gradient coil in the following. It had 128 windings and created, at the position of the atoms, a gradient of $2.25 \mathrm{G} /(\mathrm{cm} \cdot \mathrm{A})$ at an offset field of $3 \mathrm{G} / \mathrm{A}$. The coil was placed 


\section{A. Experimental details}

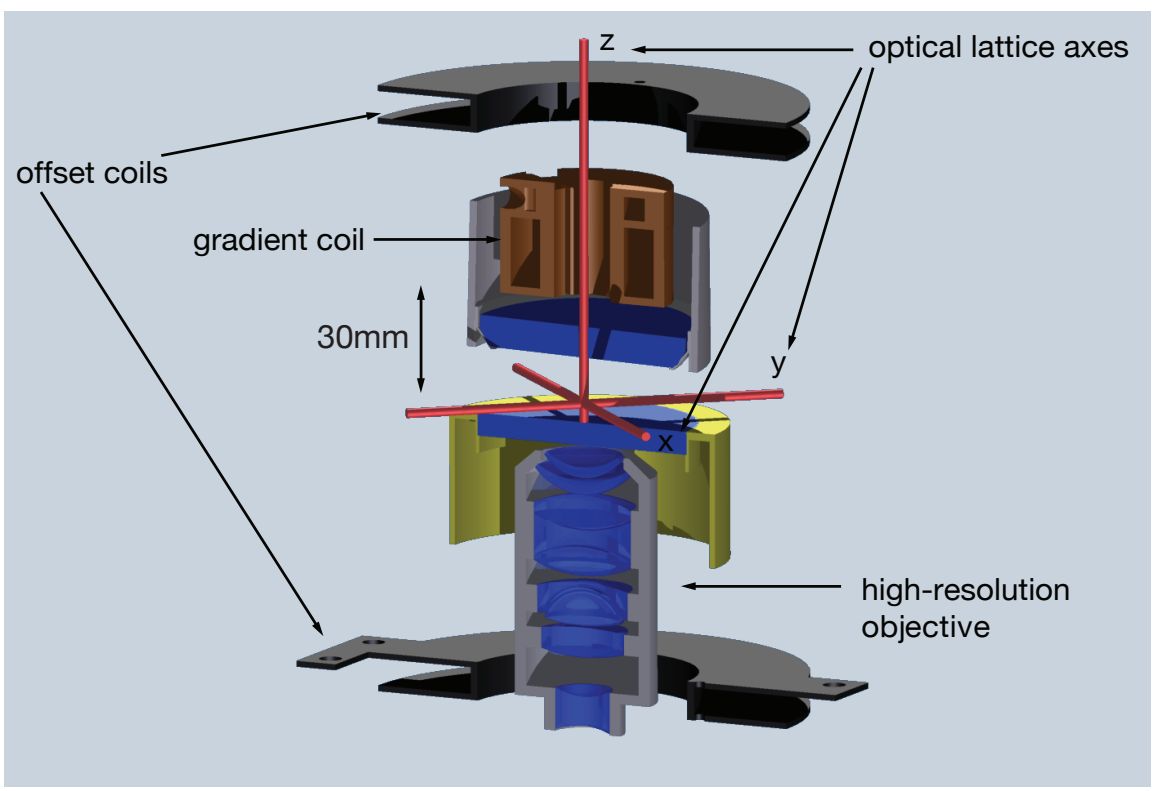

Figure A.3.: Setup including coils. 3d drawing of the high-resolution objective, the gradient coil, the pair of vertical offset coils, and the two vacuum windows of the vertical axis. For orientation, the optical lattice axes are included in red. Only the holders of the coils are shown (without the wires). Additional offset coils for the horizontal directions are not shown.

in a water-cooled housing. The current supply was High Finesse, UCS 20/15.

To achieve a high stability of the current, we employed an additional current stabilization. For this, we measured the current through the gradient coil using a current transducer (Danfysik IT 60-S). We stabilized the current with a transistor operated in parallel to the gradient coil. The resulting relative stability of the current was on the order of $10^{-5}$.

We increased the vertical gradient exponentially in $1 \mathrm{~s}$ to $\partial B / \partial z=45 \mathrm{G} / \mathrm{cm}$. This resulted in a frequency gradient for the $\left|F=1, m_{F}=-1\right\rangle$ to $\left|F=2, m_{F}=-2\right\rangle$ transition of $\partial \nu / \partial z=9.5 \mathrm{kHz} / \mu \mathrm{m}$. We used additional pairs of offset coils in horizontal directions to minimize residual gradients in horizontal direction. To achieve this, also a careful initial spatial alignment of the gradient coil was necessary.

We now turn to the actual preparation procedure. First, we transferred the atoms in all antinodes from $\left|F=1, m_{F}=-1\right\rangle$ to $\left|F=2, m_{F}=-2\right\rangle$ using a broad MW-sweep. To avoid any remaining population in $\left|F=1, m_{F}=-1\right\rangle$, we applied an additional laser pulse on the repump transition $5 S_{1 / 2}, F=1$ to $5 P_{3 / 2}, F=2$.

Afterwards, we applied a position selective HS1 MW-pulse [182] to transfer the atoms in a single antinode from $\left|F=2, m_{F}=-2\right\rangle$ to $\left|F=1, m_{F}=-1\right\rangle$. The pulse had a duration of $5 \mathrm{~ms}$ and a sweep width of $3.5 \mathrm{kHz}$. The remaining atoms in state $\left|F=2, m_{F}=-2\right\rangle$ were removed from the trap by a laser pulse resonant with the $5 S_{1 / 2}, F=2$ to $5 P_{3 / 2}, F=3$ transition. 

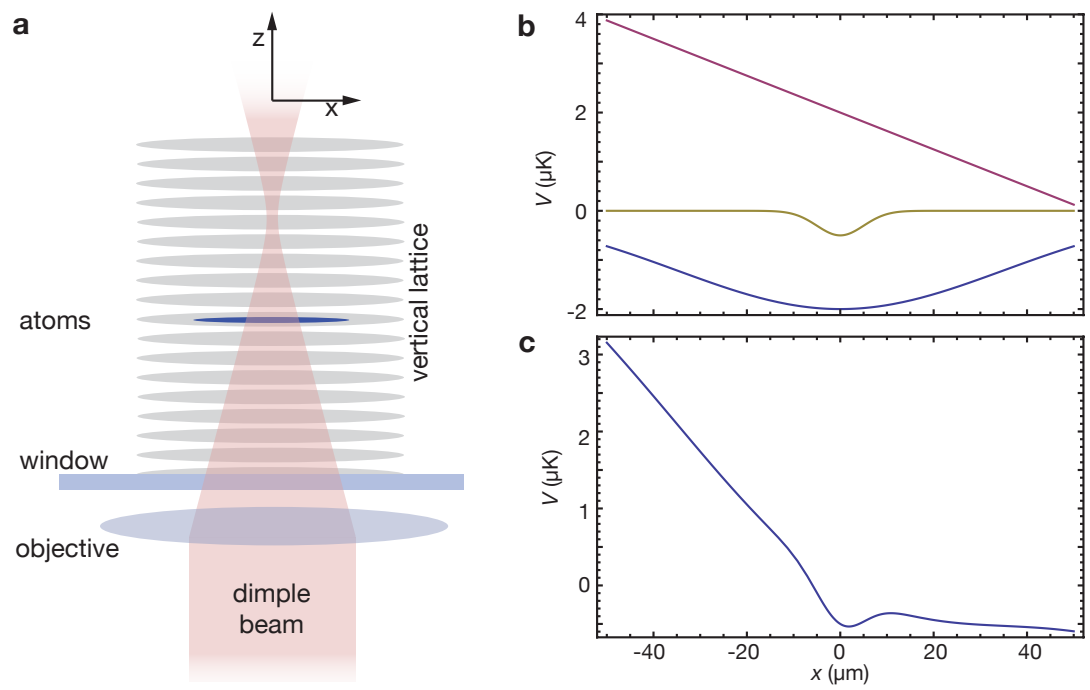

Figure A.4.: Dimple trap and potential during the final evaporation stage. a, Sketch of the dimple beam. The beam for the dimple trap is shone in through the high-resolution objective (represented as a single lens) with its focus approximately $50 \mu \mathrm{m}$ above the atom cloud resulting in a beam waist of about $10 \mu \mathrm{m}$ in the plane of the atoms. $\mathbf{b}$, Conservative potential created by the vertical lattice (blue), the dimple trap (yellow), and the magnetic gradient (purple) as a function of the horizontal distance $x$. c, Sum of the potentials shown in $\mathbf{b}$. Without the dimple trap the potential would be completely tilted and all atoms would escape horizontally.

\section{Magnetic field during final evaporation}

For the final evaporation in the single antinode, we employed a magnetic gradient in horizontal direction of $11.25 \mathrm{G} / \mathrm{cm}$ in order to spill out atoms in the horizontal direction (Fig. A.4). To achieve this, we used a pair of offset coils in vertical direction in addition to the gradient coil (Fig. A.3). The purpose of this offset coils is to shift the zero point of the magnetic field into the $x-y$-plane of the atom cloud. For this, the offset field created by this pair of coils has to compensate the offset field created by the gradient coil. Additionally, we used offset coils in horizontal direction to position the magnetic zero outside of the atom cloud within the same $x$-y-plane. This configuration resulted in an approximately linear field gradient in horizontal direction across the atom cloud.

To achieve this final configuration, we first reduced the current in the gradient coil from $20 \mathrm{~A}$ to $8 \mathrm{~A}$ within $100 \mathrm{~ms}$ following an s-shaped function. Then, we increased the current in the pair of offset coils to yield an offset magnetic field of $24 \mathrm{G}$, approximately compensating the offset field created by the gradient coil and resulting in the aforementioned field geometry. This consisted of a two step process with a first increase to $20 \mathrm{G}$ in $100 \mathrm{~ms}$ and a second increase to $24 \mathrm{G}$ in $500 \mathrm{~ms}$ following an inverted 


\section{A. Experimental details}
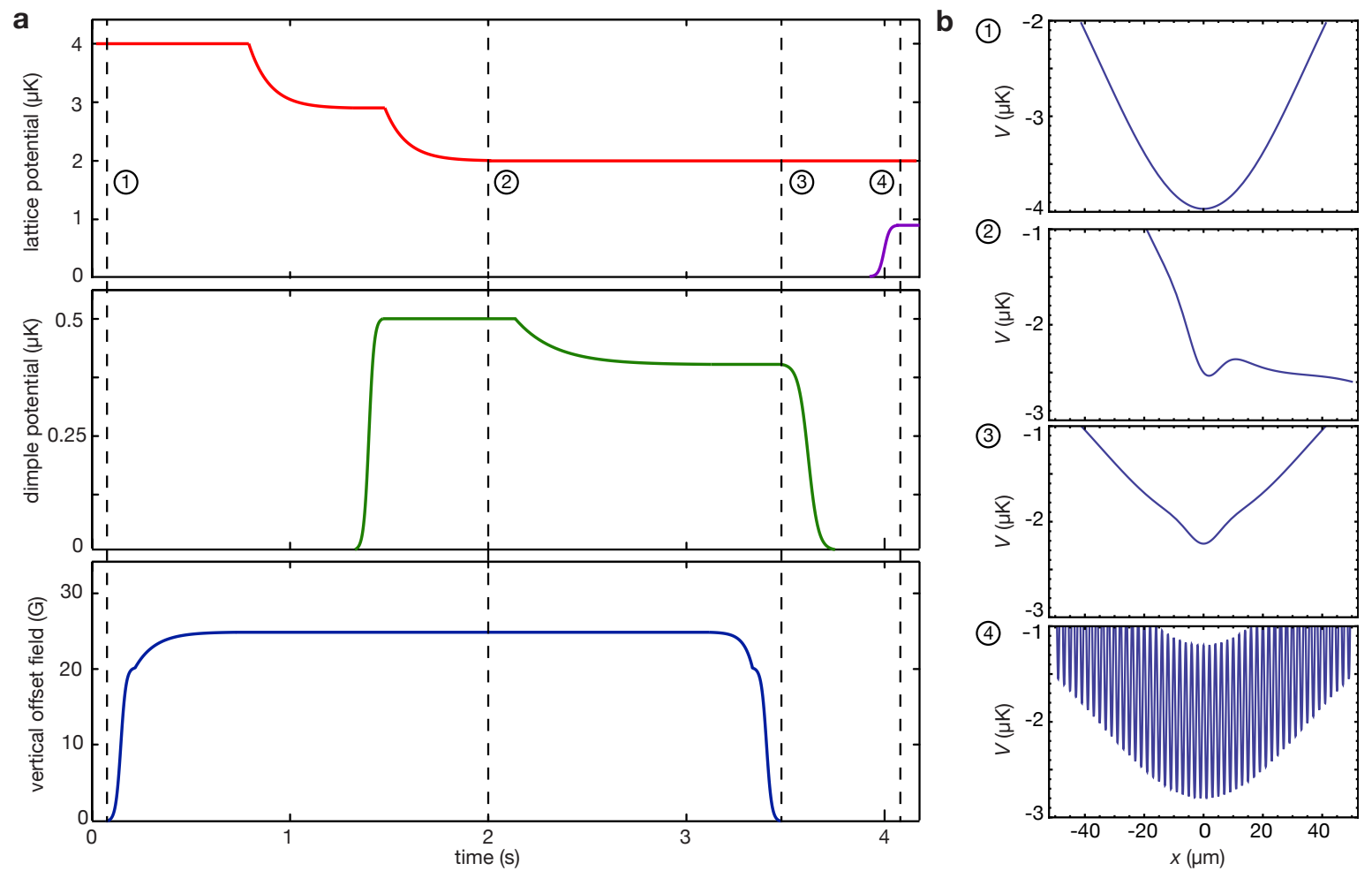

Figure A.5.: Sequence during final part of the evaporation and adiabatic lattice loading. a, Top panel: Optical lattice potential of the $z$-lattice axis during the evaporation in a single antinode (red). The lattice ramp for the adiabatic loading of the horizontal lattice axes (purple) after the evaporation sequence is also included. Middle panel: Depth of the dimple trap. Bottom panel: Magnetic field created by the pair of offset coils in vertical direction. The latter controls the strength of the gradient in horizontal direction. $\mathbf{b}$, Combined optical and magnetic potentials at times during the sequence labeled with corresponding numbers and vertical dashed lines in a. The depths of the optical potentials in a denote the depths of the individual potentials without the gradient. The lattice spacing of the horizontal lattice axis in the bottom panel of $\mathbf{b}$ is shown four times larger compared to the actual experimental value in order to make the periodic modulation visible on the scale of the figure.

exponential function (Fig. A.5).

\section{Dimple trap and final evaporation}

To increase the atom number stability, we used an additional dimple trap [186]. It was created by a laser beam focused through the high-resolution objective. The focus of the beam was positioned $50 \mu \mathrm{m}$ above the atom cloud with a waist of $w_{0}=1.5 \mu \mathrm{m}$. 


\section{A.2. Optical lattices and high-resolution imaging}

The antinode, in which the atoms reside, cuts the beam transversally and the beam had a waist of about $10 \mu \mathrm{m}$ at this position (Fig. A.4).

For the dimple trap, we used a laser diode with wavelength $850 \mathrm{~nm}$ (JDS Uniphase, SDL-5411-G1, bandwidth $3 \mathrm{~nm}$ ) that we amplified with a tapered amplifier (M2K, TA0850-1500-CM). The beam was fiber-coupled and intensity stabilized using an AOM. Before shining in the dimple laser, we exponentially reduced the depth of the vertical lattice from $40 E_{r}$ to $30 E_{r}$ in $500 \mathrm{~ms}$ (Fig. A.5). Then, we increased the dimple laser power to its final value within $100 \mathrm{~ms}$. This value was optimized such that a small portion of the atoms is lost due to three-body recombination of the dense cloud in the dimple trap. This resulted in an effective stabilization of the atom number at this stage of the sequence.

Then, we further reduced the depth of the vertical lattice to $20 E_{r}$ in $500 \mathrm{~ms}$. Without the dimple trap, all atoms would be spilled out of the trap horizontally in this configuration (panel 2 in Fig. A.5b). As a result, we kept only atoms in the dimple trap.

The last step of the evaporation was achieved by reducing the power of the dimple trap to about $80 \%$ of its maximum value. Adjustment of the final dimple-trap power allowed for a fine-adjustment of the final atom number.

We then removed the tilt in horizontal direction by decreasing the current in the vertical offset coil to zero within $300 \mathrm{~ms}$. Following this, we reduced the power of the dimple trap to zero within $200 \mathrm{~ms}$.

In this final configuration the atoms were held only by the vertical optical lattice. This was followed by the adiabatic loading to the horizontal lattice axes (see also Sec.3). The magnetic gradient in $z$-direction is kept on for the remaining sequence (until the in situ fluorescence detection).

The total preparation sequence took approximately $20 \mathrm{~s}$.

\section{A.2. Optical lattices and high-resolution imaging}

\section{Optical lattice setup}

We first describe the setup for the lattice axes in $x$ and $y$-directions. For each axis, we used a separate fiber amplifier (Nufern, Nuamp 50W). Both amplifiers were seeded with the same single-frequency solid-state laser (Innolight, Mephisto $2 \mathrm{~W}$ ). In the following, we describe only one of the two axes as the setup for both axes was nearly identical. After the amplification fiber, we reduced the beam diameter with a 3:1 telescope, sent it through an optical isolator (Optics for Research IO-5-1064-VHP), sent the beam through an AOM (Crystal Technology, 3110-197), and coupled it to an additional fiber (ams Technologies). The AOM was used for intensity stabilization up to a maximal lattice depth of $80 E_{r}$. For the very deep lattice depths of $3000 E_{r}$, during the imaging phase, we had no intensity stabilization. After the last fiber, we typically achieved a power of about $10 \mathrm{~W}$. 


\section{A. Experimental details}

After the fiber output, we sent the beam through another optical isolator (Linos-FI1060-STI) and a telescope with a $f=50 \mathrm{~mm}$ and a $f=60 \mathrm{~mm}$ lens. We adjusted the distance between both lenses to fine-tune the beam size at at the position of the atom cloud. The beam was focused onto the atoms with a $f=200 \mathrm{~mm}$ lens. A $f=50 \mathrm{~mm}$ lens focused the beam onto the mirror used to retro-reflect the beam. In this configuration the position of the retro-reflected beam at the atom cloud is insensitive to the tilt of the mirror.

For the lattice in $z$-direction, we used a high-power solid-state laser (Innolight Mephisto MOPA 18W). The remaining setup is similar to the horizontal axes. The main difference is that the retro-reflection stems from the vacuum window in front of the high-resolution objective.

\section{Determination of the lattice depths and Bose-Hubbard parameters}

In order to calibrate the lattice depths, we modulated the lattice intensity and measured parametric excitations of the atoms from the zeroth to the second Bloch band. As a result, we observed a resonance in the in situ width of the atom cloud as a function of the modulation frequency. Our method allowed us to measure the transition frequency with an uncertainty of $1-2 \%$. From the lattice depths, determined in this way, we calculated the Bose-Hubbard parameters $J$ and $U$ using a numerical band structure calculation (Sec. 2.4).

During the measurements of correlation functions in Part II, we saw drifts of the lattice depth of typically $5 \%$. Both errors together lead to an experimental uncertainty of $\sim 10 \%$ for $J / U$.

For the measurements described in Part III, we tried to minimize drifts of the lattice depths by repeating the calibration for the horizontal lattice axes after 80 experimental runs. We observed a drift of the lattice depths between these calibrations of maximally $2 \%$, but in most cases no change was observable.

\section{High-resolution imaging}

A detailed account of the high-resolution imaging-setup and -sequence can be found in Ref. [74] and we only describe changes here. The changes apply to the measurements in Part II and III.

For the imaging, we increased the lattices depths in all directions with a first ramp to $80 E_{r}$ and a second ramp to $3000 E_{r}$. We decreased the duration of the first exponential ramp to $0.2 \mathrm{~ms}$ to make sure that the ramp does not cause any local number-squeezing dynamics [16].

The high-resolution objective has a rather short depth of focus of about $1 \mu \mathrm{m}$. During our measurements, we saw drifts of the imaging system relative to the single 
antinode, in which the atoms are trapped, that were larger than this value resulting in defocused images. In general, we could compensate for such drifts by moving the objective in vertical direction using a high-precision ring-piezo mount (PIFOC P-726, Physik Instrumente). To avoid repeated manual refocusing, we implemented an auto-focus procedure. The auto-focusing is performed during a sequence that is repeated constantly while the system is not used for measurements. This sequence prepares cold clouds and images them (in the same way as the normal sequences used for the actual measurements) in order to keep the system thermally stable. In this sequence, we took up to seven pictures of the same cloud with different vertical positions of the objective (with a reduced illumination time for each picture). We analyzed these pictures to find the optimum objective position and used the found value automatically in the subsequent measurement sequences. 
A. Experimental details 


\section{B. Numerical methods for correlation functions}

The author of this thesis would like to stress that the actual numerical simulations have not been performed by himself. In particular, the DMRG calculation was performed by Leonardo Mazza, the MPS calculation by Mari Carmen Banuls, and the QMC calculation by Lode Pollet (see Ref. [29] for further details).

\section{Density-Matrix Renormalization Group (DMRG) and finite-size scaling}

We use a DMRG code with open boundary conditions, which implements the conservation of the number of particles. The considered system sizes span between $L=168$ and $L=256$. The number of retained states $m$ has been chosen in order to have a truncation error smaller than $10^{-8}$ [187]. This results in a relative error for the shown data which is smaller than $10^{-3}$, as estimated via comparison of data for different $m$ between 185 and 235 .

The string correlator is strongly affected by the presence of boundaries and finite-size effects. We introduce the notation $O_{P}^{2}(J / U, l, L)$ to address the expectation value of the string correlator of length $l$ obtained from the numerical simulation of a system of length $L$. The $l$ sites are always taken in the center of the system to minimize boundary effects. For the finite size scaling in the inset of Fig. 6.2, we analyzed correlators $O_{P}^{2}(J / U, \alpha L, L)$ for a fixed fraction $\alpha$ of the total length $L(l=\alpha L)$. We extrapolated $O_{P}^{2}(J / U, \alpha)=\lim _{L \rightarrow \infty} O_{P}^{2}(J / U, \alpha L, L)$ using a scaling of the form $O_{P}^{2}(J / U, \alpha L, L)=a+b / L^{\eta}[188-190]$.

To determine $(J / U)_{c}$, we fitted the extrapolated values with

\begin{tabular}{|l||c|c|c|}
\hline & $\alpha=1 / 4$ & $\alpha=1 / 3$ & $\alpha=1 / 2$ \\
\hline \hline$\left[\left(\frac{J}{U}\right)_{1},\left(\frac{J}{U}\right)_{2}\right]=[0.23,0.37]$ & 0.303 & 0.306 & 0.296 \\
\hline$\left[\left(\frac{J}{U}\right)_{1},\left(\frac{J}{U}\right)_{2}\right]=[0.24,0.36]$ & 0.310 & 0.311 & 0.299 \\
\hline$\left[\left(\frac{J}{U}\right)_{1},\left(\frac{J}{U}\right)_{2}\right]=[0.25,0.35]$ & 0.319 & 0.318 & 0.305 \\
\hline$\left[\left(\frac{J}{U}\right)_{1},\left(\frac{J}{U}\right)_{2}\right]=[0.26,0.34]$ & 0.321 & 0.319 & 0.311 \\
\hline$\left[\left(\frac{J}{U}\right)_{1},\left(\frac{J}{U}\right)_{2}\right]=[0.27,0.33]$ & 0.317 & 0.314 & 0.309 \\
\hline
\end{tabular}

Table B.1.: Results for $(J / U)_{c}$ for different relative lengths $\alpha$ and fitting intervals $\left[\left(\frac{J}{U}\right)_{1},\left(\frac{J}{U}\right)_{2}\right]$. The fitting error for a given $\alpha$ and $\left[\left(\frac{J}{U}\right)_{1},\left(\frac{J}{U}\right)_{2}\right]$ is below 0.003 . 


\section{B. Numerical methods for correlation functions}

$\mathcal{O}_{P}^{2} \propto \exp \left(-A\left[(J / U)_{c}^{1 d}-(J / U)\right]^{-1 / 2}\right)$. The results for $(J / U)_{c}$ appear to be strongly dependent on the fitting interval $\left[(J / U)_{1},(J / U)_{2}\right]$ and $\alpha$, as shown in Table B.1. This large systematic error could be reduced using more advanced finite size-scaling methods [57, 189], which is beyond the scope of this manuscript. In the inset of Fig. 6.2, we show the fit using $\alpha=1 / 2$ and $\left[(J / U)_{1},(J / U)_{2}\right]=[0.25,0.35]$.

\section{Finite-temperature Matrix-Product-State (MPS) calculation}

Thermal equilibrium states for a finite chain of $q$-dimensional systems can be approximated by MPS using the techniques introduced in $[92,93]$. For a chain of length $L$, the density matrix can be expressed as a vector in a Hilbert space of larger dimension, $q^{2 L}$. We approximate this by a MPS of length $2 L$, where sites $2 k$ and $2 k+1$ correspond to the $k$-th site in the physical system. For inverse temperature $\beta=1 /\left(k_{B} T\right)$, the thermal state (up to normalization) is formally identical to the imaginary time evolution of the identity operator $\rho \approx e^{-\beta H}=e^{-\frac{\beta}{2} H} \mathbb{1} e^{-\frac{\beta}{2} H}$. To obtain the MPS representation of this operator, we use a second-order Suzuki-Trotter decomposition of the exponentials, and apply the imaginary time evolution to the initial state corresponding to the identity operator [92].

Using this method, we have simulated the thermal states of a Bose-Hubbard chain with length $L=30$, for up to $n=4$ particles per site. We used an average over systems with different total atom numbers $N$, corresponding to the typical atom number distribution in the experiment, as well as an average over the central 9 sites. The error of the numerical simulation originates from the Trotter step $\delta$ and the bond dimension parameter $D$, which controls the number of parameters in the MPS ansatz. Both errors can be reduced by running simulations with increasing (decreasing) $D(\delta)$. We have used $D=20, \delta=0.05$, for which the estimated error is below $10^{-3}$.

\section{Quantum Monte Carlo calculations}

Numerical results at finite temperature are obtained by using the Quantum Monte Carlo worm algorithm [191] in the implementation of Refs. [41, 192]. This is an unbiased and statistically exact path integral Monte Carlo algorithm, formulated in continuous imaginary time, in which two worm operators (corresponding to $\hat{a}$ and $\hat{a}^{\dagger}$ operators) perform local updates in an extended configuration space and hereby directly sample the Green function. These updates lead to a fast decorrelation between the configurations resulting in an integrated autocorrelation time of order unity. A bootstrap analysis shows that the relative error is below $10^{-3}$ for the presented data. It was previously demonstrated that experiments on the Bose-Hubbard model are in one-to-one agreement with such first principles simulations for realistic system sizes 
(on the order of a million atoms) and temperatures [53]. In our simulations, we assume that temperature scales as $T \sim U$ which was shown to be a good assumption in this parameter regime [193]. 
B. Numerical methods for correlation functions 


\section{Calculation for non-local correlations in one dimension}

We evaluate the string order parameter in certain limits of the Sine-Gordon model (Eq. 6.20)

$$
H_{S G}=\frac{1}{2 \pi} \int_{-\infty}^{+\infty} d x\left[v K(\pi \Pi(x))^{2}+\frac{v}{K}\left(\frac{\partial \phi(x)}{\partial x}\right)^{2}-g \cos (2 \phi(x))\right] .
$$

We will set $\hbar=1$ and drop the hat notation for operators. $K$ is the Luttinger liquid parameter, and $v$ the sound velocity in the gapless phase, and $g$ describes the strength of the lattice potential. The fields $\phi$ and $\Pi$ are canonically conjugate variables that satisfy

$$
\left[\phi(x), \Pi\left(x^{\prime}\right)\right]=i \delta\left(x-x^{\prime}\right) .
$$

The string order parameter is given by

$$
\begin{aligned}
\mathcal{O}_{P}^{2}(l) & \propto\langle\cos (\phi(x)) \cos (\phi(x+l))\rangle \\
& \propto\left\langle e^{i(\phi(x+l)-\phi(x))}\right\rangle+\left\langle e^{-i(\phi(x+l)-\phi(x))}\right\rangle+\left\langle e^{i(\phi(x+l)+\phi(x))}\right\rangle+\left\langle e^{-i(\phi(x+l)+\phi(x))}\right\rangle .
\end{aligned}
$$

In the following, we will concentrate on limits, where $\hat{H}_{S G}$ is quadratic in the fields and where $\langle\phi(x)\rangle=0$. In this case, the following equality holds (see, e.g., Refs. $[111,136])$

$$
\begin{aligned}
\left\langle e^{ \pm i(\phi(x+l) \pm \phi(x))}\right\rangle & =e^{-\frac{1}{2}\left\langle(\phi(x+l) \pm \phi(x))^{2}\right\rangle} \\
& =e^{-\left(\left\langle\phi(0)^{2}\right\rangle \pm\langle\phi(l) \phi(0)\rangle\right)},
\end{aligned}
$$

where in the last step we made use of the translation invariance of the system. With this, we find

$$
\mathcal{O}_{P}^{2}(l) \propto e^{-\left(\left\langle\phi(0)^{2}\right\rangle-\langle\phi(l) \phi(0)\rangle\right)}+e^{-\left(\left\langle\phi(0)^{2}\right\rangle+\langle\phi(l) \phi(0)\rangle\right) .}
$$

Thus, we have to calculate expectations values of the form $\left\langle\phi(0)^{2}\right\rangle \pm\langle\phi(l) \phi(0)\rangle$.

To simplify the calculation, we rescale

$$
\begin{array}{r}
\phi \rightarrow K^{1 / 2} \phi \\
\Pi \rightarrow \frac{1}{K^{1 / 2}} \Pi
\end{array}
$$




\section{Calculation for non-local correlations in one dimension}

We thus have

$$
H_{S G}=\frac{1}{2 \pi} \int_{-\infty}^{+\infty} d x\left[v(\pi \Pi(x))^{2}+v\left(\frac{\partial \phi(x)}{\partial x}\right)^{2}-g \cos \left(2 K^{1 / 2} \phi(x)\right)\right]
$$

and

$$
\mathcal{O}_{P}^{2}(l) \propto e^{-K\left(\left\langle\phi(0)^{2}\right\rangle-\langle\phi(l) \phi(0)\rangle\right)}+e^{-K\left(\left\langle\phi(0)^{2}\right\rangle+\langle\phi(l) \phi(0)\rangle\right)} .
$$

\section{Gapless phase}

We first study the behavior of the string order parameter for $g=0$. The Hamiltonian C.10 in this limit is the standard Luttinger Liquid Hamiltonian

$$
H_{L L}=\frac{1}{2 \pi} \int_{-\infty}^{+\infty} d x\left[v(\pi \Pi(x))^{2}+v\left(\frac{\partial \phi(x)}{\partial x}\right)^{2}\right]
$$

which is equivalent to the Hamiltonian of a quantized harmonic string. We can diagonalize $H_{L L}$ by

$$
\begin{array}{r}
\phi(x)=\int d q \frac{1}{2|q|^{1 / 2}}\left(a_{q}+a_{-q}^{\dagger}\right) e^{i q x} \\
\Pi(x)=\int d q \frac{-i|q|^{1 / 2}}{2 \pi}\left(a_{q}-a_{-q}^{\dagger}\right) e^{i q x}
\end{array}
$$

where $a_{q}^{\dagger}\left(a_{q}\right)$ is the creation (annihilation) operator for the normal mode with momentum $q$. The pre-factors are chosen such that:

$$
\left[a_{q^{\prime}}, a_{q}^{\dagger}\right]=\delta\left(q^{\prime}-q\right)
$$

With this, we find

$$
H_{L L}=\int d q v|q|\left(a_{q}^{\dagger} a_{q}+\frac{1}{2}\right)
$$

showing a gapless spectrum with a linear dispersion relation $v|q|$.

We are interested in using Eq. C.13 to calculate the behavior of the string order parameter for the ground state of the system at zero-temperature. The expectation values in Eq. C.11 are therefore to be taken in the groundstate of the system $|0\rangle$.

Using Eq. C.13, we have

$$
\left\langle\phi(0)^{2}-\phi(l) \phi(0)\right\rangle=\left\langle 0\left|\int d q^{\prime} \int d q \frac{1}{4\left|q^{\prime}\right|^{1 / 2}|q|^{1 / 2}}\left(a_{q^{\prime}}+a_{-q^{\prime}}^{\dagger}\right)\left(a_{q}+a_{-q}^{\dagger}\right)\left(1-e^{i q l}\right)\right| 0\right\rangle
$$


Because of $a_{q}|0\rangle=0$ (for arbitrary $q$ ), only the combination $a_{q^{\prime}} a_{-q}^{\dagger}=\delta\left(q^{\prime}+q\right)+a_{q^{\prime}}^{\dagger} a_{-q}$ yields a non-zero expectation value. We therefore find

$$
\left\langle\phi(0)^{2}-\phi(l) \phi(0)\right\rangle=\int d q \frac{1}{4|q|}\left(1-e^{i q l}\right)=\int_{0}^{\infty} d q \frac{1}{2|q|}(1-\cos (q l)) .
$$

The integral in this form does not converge. However, we are dealing with an effective continuum description of a lattice system and momenta have to be limited to $q \lesssim 1 / a$, where $a$ is of the order of of the lattice constant. We can solve this problem by introducing a smooth momentum cutoff $e^{-q a}$ and find

$$
\left\langle\phi(0)^{2}-\phi(l) \phi(0)\right\rangle=\int_{0}^{\infty} d q e^{-q a} \frac{1}{2|q|}\left(1-\cos (q l)=\frac{1}{4} \log \left(1+\left(\frac{l}{a}\right)^{2}\right) .\right.
$$

The term $\left\langle\phi(0)^{2}+\phi(l) \phi(0)\right\rangle$ yields after a similar calculation

$$
\left\langle\phi(0)^{2}+\phi(l) \phi(0)\right\rangle=\int d q e^{-q a} \frac{1}{2|q|}(1+\cos (q l)),
$$

which is divergent for all $l$ and therefore $e^{-K\left(\left\langle\phi(0)^{2}\right\rangle+\langle\phi(l) \phi(0)\rangle\right)}=0$. In a finite size system, however, the $q$ integral should also have a low-momentum cutoff. Implementing such a cut-off, the integral can be performed and $e^{-K\left(\left\langle\phi(0)^{2}\right\rangle+\langle\phi(l) \phi(0)\rangle\right)}$ yields a finitesize correction to the string order parameter. We do not study this here and assume that the system size is much larger than $a$, such that $e^{-K\left(\left\langle\phi(0)^{2}\right\rangle+\langle\phi(l) \phi(0)\rangle\right)} \approx 0$. Summing up, we find in the gapless phase

$$
\mathcal{O}_{P}^{2}(l) \propto e^{-K\left(\left\langle\phi(0)^{2}\right\rangle-\langle\phi(l) \phi(0)\rangle\right)} \approx\left(1+\left(\frac{l}{a}\right)^{2}\right)^{-\frac{K}{4}} .
$$

Thus, we have an algebraic decay of the string order parameter with $l^{-K / 2}$. In particular, we find

$$
\mathcal{O}_{P}^{2}=\lim _{l \rightarrow \infty} \mathcal{O}_{P}^{2}(l)=0 .
$$




\section{Calculation for non-local correlations in one dimension}

\section{Gapped phase in harmonic approximation}

For small $K$, we can use a harmonic approximation of the cosine term in $H_{S G}$ (Eq. C.10) and find

$$
H_{S G} \approx \frac{1}{2 \pi} \int_{-\infty}^{+\infty} d x\left[v(\pi \Pi(x))^{2}+v\left(\frac{\partial \phi(x)}{\partial x}\right)^{2}+2 g K \phi(x)^{2}\right],
$$

where we have subtracted a constant offset $g$. We can diagonalize this by setting

$$
\begin{array}{r}
\phi(x)=\int d q \frac{v^{1 / 2}}{2 \omega_{q}^{1 / 2}}\left(a_{q}+a_{-q}^{+}\right) e^{i q x} \\
\Pi(x)=\int d q \frac{-i \omega_{q}^{1 / 2}}{2 \pi v^{1 / 2}}\left(a_{q}-a_{-q}^{\dagger}\right) e^{i q x},
\end{array}
$$

where $\omega_{q}=\left(2 v g K+(v q)^{2}\right)^{1 / 2}$, and find

$$
H_{S G} \approx \int d q \omega_{q}\left(a_{q}^{\dagger} a_{q}+\frac{1}{2}\right) .
$$

We, therefore, find an energy gap $m=(2 v g K)^{1 / 2}$ and a quadratic dispersion relation for small momenta.

The calculation of the correlation functions can be performed in a similar manner as in the gapless phase. We find

$$
\left\langle\phi(0)^{2} \pm \phi(l) \phi(0)\right\rangle=\int d q e^{-q a} \frac{1}{2\left(\tilde{m}^{2}+q^{2}\right)^{1 / 2}}(1 \pm \cos (q l)),
$$

where we set $\tilde{m}=m / v$. We can perform the integral $\int d q \frac{1}{\left(\tilde{m}^{2}+q^{2}\right)^{1 / 2}} \cos (q l)=K_{0}(\tilde{m} l)$ without momentum cutoff, where we defined $K_{0}(x)$ as the zeroth modified Bessel function of second kind.

The integral $\int d q e^{-q a} \frac{1}{\left(\tilde{m}^{2}+q^{2}\right)^{1 / 2}}$ can also be performed analytically yielding a combination of several special functions. In this case, it is easier to impose a hard momentum cut-off $1 / a$ and perform $\int_{0}^{1 / a} d q \frac{1}{\left(\tilde{m}^{2}+q^{2}\right)^{1 / 2}}=A(\tilde{m} a)$, where $A(x)$ is the inverse hyperbolic cosecant.

With this, we find

$$
\left\langle\phi(0)^{2} \pm \phi(l) \phi(0)\right\rangle \approx \frac{1}{2}\left(A(\tilde{m} a) \pm K_{0}(\tilde{m} l)\right),
$$

In total, we have

$$
\begin{aligned}
\mathcal{O}_{P}^{2}(l) & \propto e^{-K\left(\left\langle\phi(0)^{2}\right\rangle-\langle\phi(l) \phi(0)\rangle\right)}+e^{-K\left(\left\langle\phi(0)^{2}\right\rangle+\langle\phi(l) \phi(0)\rangle\right)} \\
& \approx e^{-\frac{K}{2} A(\tilde{m} a)}\left(e^{+\frac{K}{2} K_{o}(\tilde{m} l)}+e^{-\frac{K}{2} K_{o}(\tilde{m} l)}\right) \\
& =2 e^{-\frac{K}{2} A(\tilde{m} a)} \cosh \left(\frac{K}{2} K_{o}(\tilde{m} l)\right)
\end{aligned}
$$


We can simplify this further, by noting that for small $x$, we have $A(x) \approx$ $1 / 2(\log [4]-2 \log [x])$. If we assume $\tilde{m} a$ to be small, we get

$$
\mathcal{O}_{P}^{2}(l) \approx\left(\frac{\tilde{m} a}{2}\right)^{K / 2} \cosh \left(\frac{K}{2} K_{o}(\tilde{m} l)\right) .
$$

Let us inspect some of the important features of this expression. First, we are interested in the long-distance limit. We know that $\lim _{x \rightarrow \infty} K_{0}(x)=0$. Therefore, the string order parameter is

$$
\lim _{l \rightarrow \infty} \mathcal{O}_{P}^{2}(l) \propto \tilde{m}^{K / 2},
$$

highlighting an algebraic dependence of the order parameter on the gap of the system. In particular, the string order parameter is non-zero for $m>0$ in the gapped phase. We would like to note that the exponent of $K / 2$ also appears if the $\int d q \frac{1}{\left(\tilde{m}^{2}+q^{2}\right)^{1 / 2}}$ was performed with a smooth momentum cutoff.

Let us study the long-distance behavior in more detail. For large $x, K_{0}(x) \approx e^{-x} \sqrt{\frac{\pi}{2 x}}$ is small and we can expand $\cosh (y)=1+y^{2} / 2$. This gives us

$$
\mathcal{O}_{P}^{2}(l) \approx\left(\frac{\tilde{m} a}{2}\right)^{K / 2}\left(1+\frac{\pi K^{2}}{16} e^{-2 \tilde{m} l} \frac{1}{\tilde{m} l}\right)
$$

for the long-distance behavior. We therefore find an essentially exponential decay to a constant value, with a correlation length proportional to $1 / \tilde{m}$.

For short distances, we have $K_{0}(x) \approx$ const. $-\log (\mathrm{x})$ and therefore $\mathcal{O}_{P}^{2}(l) \propto l^{-K / 2}$. Thus, we recover the behavior of the gapless phase. The full form $\mathcal{O}_{P}^{2}(l) \propto \cosh \left(\frac{K}{2} K_{o}(\tilde{m} l)\right)$ interpolates between the short-distance algebraic decay and the long-distance exponential decay. The transition between both forms happens on a length-scale proportional to $1 / \tilde{m}$. We therefore observe that for a decreasing gap value $\tilde{m}$, the algebraic decay becomes increasingly dominant. 
C. Calculation for non-local correlations in one dimension 


\section{Calculation for non-local correlations in two dimensions}

We perform a calculation of the non-local correlation function $\mathcal{O}^{2}(A)$ defined in Eq. 7.3. Our starting point is the the dual Hamiltonian

$$
\hat{H}_{d u a l} \propto \int_{\vec{r}}\left[v K \vec{E}^{2}+\frac{v}{K}(\nabla \times \vec{A})^{2}-g \cos \left(A_{x}\right)-g \cos \left(A_{y}\right)\right],
$$

which is a continuum version of the Hamiltonian derived in the main text (Eq. 7.36). $\vec{E}$ and $\vec{A}$ are two-dimensional vectors of operators, where we dropped the hat notation for operators. We introduced different pre-factors in the Hamiltonian to simplify the notation and to make similarities to the one-dimensional model (Eq. 6.20) more apparent.

The dual theory is completed by the constraint

$$
\vec{\nabla} \vec{E}(r)=N(r)
$$

on the Hilbert-space (see Eq. 7.32), which is similar to the Gauss law in ordinary electromagnetism [194].

In the following calculation, we do not keep track of overall constant pre-factors of $\pi$ etc., in particular related to delta-functions. We will solve the problem in a harmonic approximation of the cosine-terms in the Hamiltonian. In this approximation, we have up to constant energies

$$
\hat{H}_{d u a l} \propto \int_{\vec{r}}\left[v K \vec{E}^{2}+\frac{v}{K}(\nabla \times \vec{A})^{2}+2 g \vec{A}^{2}\right]
$$

Our analysis is simplest in Fourier space using the transformations

$$
\begin{aligned}
& \vec{A}(r)=\int_{k} \vec{A}(k) e^{i k r} \\
& \vec{E}(r)=\int_{k} \vec{E}(k) e^{i k r} .
\end{aligned}
$$

Note that $k$ and $r$ are vectors. We find the Hamiltonian

$$
\hat{H}_{d u a l} \propto \int_{k}\left[v K \vec{E}(k) \vec{E}(-k)+\frac{v}{K}(k \times \vec{A}(k))(k \times \vec{A}(-k))+2 g \vec{A}(k) \vec{A}(-k)\right]
$$

and the Gauss law

$$
i k \cdot \vec{E}(k)=N(k)
$$




\section{Calculation for non-local correlations in two dimensions}

in Fourier space.

We can split the vector $\vec{A}$ in a transversal $\vec{A}_{t}(k)$ and longitudinal component $\vec{A}_{l}(k)$, which are defined by the properties

$$
\begin{array}{r}
k \cdot \vec{A}_{t}(k)=0 \\
k \times \vec{A}_{l}(k)=0,
\end{array}
$$

and perform a similar splitting for $\vec{E}(k)$ [194].

We find that, as a consequence, the Hamiltonian splits into a transverse part $\hat{H}_{t}$ and a longitudinal part $\hat{H}_{l}$ :

$$
\begin{aligned}
\hat{H}_{d u a l} & \propto \hat{H}_{l}+\hat{H}_{t} \\
\hat{H}_{l} & =\int_{k}\left[v K \vec{E}_{l}(k) \vec{E}_{l}(-k)+2 g \vec{A}_{l}(k) \vec{A}_{l}(-k)\right] \\
\hat{H}_{t} & =\int_{k}\left[v K \vec{E}_{t}(k) \vec{E}_{t}(-k)+\frac{v}{K}\left(k \times \vec{A}_{t}(k)\right)\left(k \times \vec{A}_{t}(-k)\right)+2 g \vec{A}_{t}(k) \vec{A}_{t}(-k)\right] .
\end{aligned}
$$

One can explicitly show that $\hat{H}_{t}$ and $\hat{H}_{l}$ commute, and they can, therefore, be simultaneously diagonalized.

The Gauss law now reads

$$
i k \cdot \vec{E}_{l}(k)=N(k) \text {. }
$$

Notice, that the Gauss law only involves the longitudinal part of $\vec{E}$. Therefore, it can be solved together with longitudinal part of the Hamiltonian $\hat{H}_{l}$ and the transverse part $\hat{H}_{t}$ is independent of the constraint.

One can solve Eq. D.13 for $E_{l}(k)=N(k) / i k$ and plug the result into $\hat{H}_{l}$, which yields $\hat{H}_{l}$ in terms of $N(k)$ and $\vec{A}_{l}$. Interestingly, the resulting term in the Hamiltonian involving $N(k)$ is proportional to $-\int_{r, r^{\prime}} \log \left(\left|r-r^{\prime}\right|\right) N(r) N\left(r^{\prime}\right)$. Since a non-zero eigenvalue of $N(r)$ physically means the presence of a vortex at $r$, this term resembles a logarithmic vortex-vortex interaction-energy.

We do not go into further details, because our focus is on the diagonalization of $\hat{H}_{t}$. For this, we introduce normal mode operators $a_{k}\left(a_{k}^{\dagger}\right)$ via

$$
\begin{aligned}
\vec{A}_{t}(k) & =i C(k)\left(a_{k}+a_{-k}^{\dagger}\right) \vec{e}_{t}(k) \\
\vec{E}_{t}(k) & =-\tilde{C}(k)\left(a_{k}-a_{-k}^{\dagger}\right) \vec{e}_{t}(k),
\end{aligned}
$$

where $\vec{e}_{t}(k)$ is a unit-vector perpendicular to $k$. The condition on the pre-factors is $2 C \tilde{C}=1$ in order to get a commutation relation $\left[a_{k}, a_{\tilde{k}}^{+}\right]=\delta(k-\tilde{k})$ for the normal 
mode operators.

The transverse Hamiltonian $\hat{H}_{t}$ is diagonalized if we set

$$
\begin{aligned}
C(k)^{2} & =\frac{K v}{2 \omega(k)} \\
\omega(k)^{2} & =\left(m^{2}+v^{2} k^{2}\right) \\
m^{2} & =2 K v g,
\end{aligned}
$$

where $\omega$ is the eigen-energy of a mode with wave-vector $k$ and $m$ is the energy gap, which is non-zero, only if $g>0$, within the harmonic approximation.

We are interested in a calculation of the loop correlation function

$$
W(A)=\left\langle e^{i \oint \vec{A} d \vec{x}}\right\rangle .
$$

Importantly, for the loop integral in the exponent, only the transverse part of $\vec{A}$ yields a non-zero value. We can, therefore, chose the longitudinal part at our will to simplify the calculation.

Within the harmonic approximation, one has [111, 136]

$$
W(A)=e^{-\frac{1}{2} \oint_{r_{1}} \oint_{r_{2}}\left\langle\vec{A}\left(r_{1}\right) d \overrightarrow{r_{1}} \vec{A}\left(r_{2}\right) d \vec{r}_{2}\right\rangle} .
$$

Our task is now to calculate the correlators $\left\langle\vec{A}\left(r_{1}\right) d \overrightarrow{r_{1}} \vec{A}\left(r_{2}\right) d \overrightarrow{r_{2}}\right\rangle$ for all relevant contributions to the integral.

To be more specific, we will chose a rectangular contour with sites along the $x$ and $y$-directions, and $d \overrightarrow{r_{1}}$ and $d \overrightarrow{r_{2}}$ are, therefore, in $x$ or $y$-direction. We are, thus, left to calculate the correlators $\left\langle A_{i}\left(r_{1}\right) A_{j}\left(r_{2}\right)\right\rangle$, where $A_{i}(r)$ is the projection of $\vec{A}(r)$ on the $x$ or $y$-axis.

For the calculation, we use the Fourier transformed fields. Our first interest is then in propagators of the form $\left\langle A_{i}\left(k_{1}\right) A_{j}\left(k_{2}\right)\right\rangle$.

To simplify the calculation, we chose a longitudinal part of $\vec{A}$ with $\vec{A}_{l}(k)=$ $C(k)\left(b_{k}+b_{-k}^{\dagger}\right) \vec{e}_{l}(k)$, where $b_{k}\left(b_{k}^{\dagger}\right)$ are normal-mode operators resulting from the diagonalization of $H_{l}, \vec{e}_{l}(k)$ is a unit-vector in the direction of $k$, and the constant $C$ is equal to the constant in front of the transverse component.

With this choice, we find

$$
\left\langle A_{i}\left(k_{1}\right) A_{j}\left(k_{2}\right)\right\rangle=C\left(k_{1}\right)^{2} \delta_{i, j} \delta\left(k_{1}+k_{2}\right)
$$

and

$$
\begin{aligned}
\left\langle A_{i}\left(r_{1}\right) A_{j}\left(r_{2}\right)\right\rangle & =\delta_{i, j} \int_{k} C(k)^{2} e^{i k\left(r_{1}-r_{2}\right)} \\
& \propto \delta_{i, j} K e^{-\tilde{m}\left|r_{2}-r_{1}\right|} \frac{1}{\left|r_{2}-r_{1}\right|}
\end{aligned}
$$




\section{Calculation for non-local correlations in two dimensions}

Here we have introduced the normalized gap $\tilde{m}=m / v$. Note, that the correlator between orthogonal components is zero. For $m>0$ the functional form is dominated by the exponential decay and therefore short-ranged. For $m=0$, we find a longer range $\frac{1}{\left|r_{2}-r_{1}\right|}$ behavior.

With this, one can obtain the full rectangular loop integral. First, we note that contributions, where $r_{1}$ and $r_{2}$ are on orthogonal sites, vanish. The dominating terms stem from contributions to the integral, where $r_{1}$ and $r_{2}$ are on the same site of the rectangle.

Note that the correlator is divergent, when $r_{1}=r_{2}$, which is an artifact, because we did not use a momentum cutoff to regularize the theory. We can fix this by setting $\left\langle A_{i}\left(r_{1}\right) A_{j}\left(r_{2}\right)\right\rangle=$ const. for $\left|r_{1}-r_{2}\right|<a$, where $a$ is the lattice constant (see, e.g., Ref.[122]). With this, we do not expect to change the long-range behavior of the loop correlation function.

Evaluating the resulting integral, one finds the dominant long-range contribution

$$
\log (W(A)) \propto-K L \log (L)
$$

for $m=0$, where $L$ is the linear extension of the contour, and

$$
\log (W(A)) \propto-K L
$$

for $m>0$. 


\section{Bibliography}

[1] I. Bloch, J. Dalibard, and S. Nascimbène. Quantum simulations with ultracold quantum gases. Nature Phys. 8, 267-276 (2012).

[2] M. Greiner, O. Mandel, T. Esslinger, T. W. Hänsch, and I. Bloch. Quantum phase transition from a superfluid to a Mott insulator in a gas of ultracold atoms. Nature 415, 39-44 (2002).

[3] I. Bloch, J. Dalibard, and W. Zwerger. Many-body physics with ultracold gases. Rev. Mod. Phys. 80, 885-964 (2008).

[4] R. Jördens, N. Strohmaier, K. Günter, H. Moritz, and T. Esslinger. A Mott insulator of fermionic atoms in an optical lattice. Nature 455, 204-207 (2008).

[5] U. Schneider, L. Hackermüller, S. Will, T. Best, I. Bloch, T. A. Costi, R. W. Helmes, D. Rasch, and A. Rosch. Metallic and insulating phases of repulsively interacting fermions in a 3D optical lattice. Science 322, 1520-1525 (2008).

[6] T. Esslinger. Fermi-Hubbard Physics with Atoms in an Optical Lattice. Annu. Rev. Cond. Mat 1, 129-152 (2010).

[7] C. A. Regal, M. Greiner, and D. S. Jin. Observation of Resonance Condensation of Fermionic Atom Pairs. Phys. Rev. Lett. 92, 040403 (2004).

[8] M. Zwierlein, C. Stan, C. Schunck, S. Raupach, A. Kerman, and W. Ketterle. Condensation of Pairs of Fermionic Atoms near a Feshbach Resonance. Phys. Rev. Lett. 92, 120403 (2004).

[9] M. Randeria, W. Zwerger, and M. Zwierlein (eds). The BCS-BEC Crossover and the Unitary Fermi Gas. Lecture Notes in Physics, Springer, Vol. 836 (2012).

[10] Y.-I. Shin, C. H. Schunck, A. Schirotzek, and W. Ketterle. Phase diagram of a two-component Fermi gas with resonant interactions. Nature 451, 689-693 (2008).

[11] N. Gemelke, X. Zhang, C.-L. Hung, and C. Chin. In situ observation of incompressible Mott-insulating domains in ultracold atomic gases. Nature 460, 995-998 (2009).

[12] S. Nascimbène, N. Navon, K. J. Jiang, F. Chevy, and C. Salomon. Exploring the thermodynamics of a universal Fermi gas. Nature 463, 1057-1060 (2010). 
[13] J. T. Stewart, J. P. Gaebler, and D. S. Jin. Using photoemission spectroscopy to probe a strongly interacting Fermi gas. Nature 454, 744-747 (2008).

[14] P. T. Ernst, S. Götze, J. S. Krauser, K. Pyka, D.-S. Lühmann, D. Pfannkuche, and K. Sengstock. Probing superfluids in optical lattices by momentum-resolved Bragg spectroscopy. Nature Phys. 6, 56-61 (2009).

[15] W. S. Bakr, J. I. Gillen, A. Peng, S. Fölling, and M. Greiner. A quantum gas microscope for detecting single atoms in a Hubbard-regime optical lattice. Nature $462,74-77$ (2009).

[16] W. S. Bakr, A. Peng, M. E. Tai, R. Ma, J. Simon, J. I. Gillen, S. Fölling, L. Pollet, and M. Greiner. Probing the superfluid-to-Mott insulator transition at the singleatom level. Science 329, 547-550 (2010).

[17] J. F. Sherson, C. Weitenberg, M. Endres, M. Cheneau, I. Bloch, and S. Kuhr. Single-atom-resolved fluorescence imaging of an atomic Mott insulator. Nature 467, 68-72 (2010).

[18] M. P. A. Fisher, P. B. Weichman, G. Grinstein, and D. S. Fisher. Boson localization and the superfluid-insulator transition. Phys. Rev. B 40, 546-570 (1989).

[19] D. Jaksch, C. Bruder, J. I. Cirac, C. Gardiner, and P. Zoller. Cold Bosonic Atoms in Optical Lattices. Phys. Rev. Lett. 81, 3108-3111 (1998).

[20] S. Sachdev. Quantum Phase Transitions. Cambridge University Press, Cambridge, 2nd ed. (2011). ISBN 0521514681.

[21] T. Gericke, P. Würtz, D. Reitz, T. Langen, and H. Ott. High-resolution scanning electron microscopy of an ultracold quantum gas. Nature Phys. 4, 949-953 (2008).

[22] P. Würtz, T. Langen, T. Gericke, A. Koglbauer, and H. Ott. Experimental demonstration of single-site addressability in a two-dimensional optical lattice. Phys. Rev. Lett. 103, 80404 (2009).

[23] C.-L. Hung, X. Zhang, N. Gemelke, and C. Chin. Slow Mass Transport and Statistical Evolution of an Atomic Gas across the Superfluid-Mott-Insulator Transition. Phys. Rev. Lett. 104, 160403 (2010).

[24] X. Zhang, C.-L. Hung, S.-K. Tung, and C. Chin. Observation of quantum criticality with ultracold atoms in optical lattices. Science 335, 1070-1072 (2012).

[25] J. Simon, W. S. Bakr, R. Ma, M. E. Tai, P. M. Preiss, and M. Greiner. Quantum simulation of antiferromagnetic spin chains in an optical lattice. Nature 472, 307-312 (2011). 
[26] W. S. Bakr, P. M. Preiss, M. E. Tai, R. Ma, J. Simon, and M. Greiner. Orbital excitation blockade and algorithmic cooling in quantum gases. Nature 480, 500-503 (2011).

[27] C. Weitenberg, M. Endres, J. F. Sherson, M. Cheneau, P. Schauß, T. Fukuhara, I. Bloch, and S. Kuhr. Single-spin addressing in an atomic Mott insulator. Nature 471, 319-324 (2011).

[28] T. Fukuhara, A. Kantian, M. Endres, M. Cheneau, P. Schauß, S. Hild, D. Bellem, U. Schollwöck, T. Giamarchi, C. Gross, I. Bloch, and S. Kuhr. Quantum dynamics of a mobile spin impurity. Nature Phys., advanced online (2013).

[29] M. Endres, M. Cheneau, T. Fukuhara, C. Weitenberg, P. Schauss, C. Gross, L. Mazza, M. C. Banuls, L. Pollet, I. Bloch, and S. Kuhr. Observation of Correlated Particle-Hole Pairs and String Order in Low-Dimensional Mott Insulators. Science 334, 200-203 (2011).

[30] M. Cheneau, P. Barmettler, D. Poletti, M. Endres, P. Schauss, T. Fukuhara, C. Gross, I. Bloch, C. Kollath, and S. Kuhr. Light-cone-like spreading of correlations in a quantum many-body system. Nature 481, 484-487 (2012).

[31] P. Schauß, M. Cheneau, M. Endres, T. Fukuhara, S. Hild, A. Omran, T. Pohl, C. Gross, S. Kuhr, and I. Bloch. Observation of spatially ordered structures in a two-dimensional Rydberg gas. Nature 490, 87-91 (2012).

[32] T. Stöferle, H. Moritz, C. Schori, M. Köhl, and T. Esslinger. Transition from a Strongly Interacting 1D Superfluid to a Mott Insulator. Phys. Rev. Lett. 92, 130403 (2004).

[33] C. Schori, T. Stöferle, H. Moritz, M. Köhl, and T. Esslinger. Excitations of a Superfluid in a Three-Dimensional Optical Lattice. Phys. Rev. Lett. 93, 240402 (2004).

[34] C. Kollath, A. Iucci, T. Giamarchi, W. Hofstetter, and U. Schollwöck. Spectroscopy of Ultracold Atoms by Periodic Lattice Modulations. Phys. Rev. Lett. 97, 050402 (2006).

[35] D. Cottingham, W.N. and Greenwood. An Introduction to the Standard Model of Particle Physics. Cambridge University Press, Cambridge, 2nd ed. (2007). ISBN 0521852498.

[36] A. V. Chubukov, S. Sachdev, and J. Ye. Theory of two-dimensional quantum Heisenberg antiferromagnets with a nearly critical ground state. Phys. Rev. B 49, 1191911961 (1994).

[37] S. Sachdev. Universal relaxational dynamics near two-dimensional quantum critical points. Phys. Rev. B 59, 14054-14073 (1999). 


\section{Bibliography}

[38] W. Zwerger. Anomalous Fluctuations in Phases with a Broken Continuous Symmetry. Phys. Rev. Lett. 92, 027203 (2004).

[39] N. H. Lindner and A. Auerbach. Conductivity of hard core bosons: A paradigm of a bad metal. Phys. Rev. B 81, 054512 (2010).

[40] D. Podolsky, A. Auerbach, and D. P. Arovas. Visibility of the amplitude (Higgs) mode in condensed matter. Phys. Rev. B 84, 174522 (2011).

[41] L. Pollet. Recent developments in Quantum Monte-Carlo simulations with applications for cold gases. arXiv:1206.0781 (2012).

[42] D. Podolsky and S. Sachdev. Spectral functions of the Higgs mode near twodimensional quantum critical points. Phys. Rev. B 86, 054508 (2012).

[43] S. Trotzky, Y.-A. Chen, A. Flesch, I. P. McCulloch, U. Schollwöck, J. Eisert, and I. Bloch. Probing the relaxation towards equilibrium in an isolated strongly correlated one-dimensional Bose gas. Nature Phys. 8, 325-330 (2012).

[44] L. Amico, A. Osterloh, and V. Vedral. Entanglement in many-body systems. Rev. Mod. Phys. 80, 517-576 (2008).

[45] A. Daley, H. Pichler, J. Schachenmayer, and P. Zoller. Measuring Entanglement Growth in Quench Dynamics of Bosons in an Optical Lattice. Phys. Rev. Lett. 109, 020505 (2012).

[46] C. Gerry and P. Knight. Introductory quantum optics. Cambridge University Press, Cambridge (2004). ISBN 052152735X.

[47] J. Hubbard. Electron Correlations in Narrow Energy Bands. Proc. R. Soc. A 276, 238-257 (1963).

[48] W. Zwerger. Mott-Hubbard transition of cold atoms in optical lattices. J. Opt. B: Quantum Semiclass. Opt. 5, 9-16 (2003).

[49] S. D. Huber, E. Altman, H. P. Buchler, and G. Blatter. Dynamical properties of ultracold bosons in an optical lattice. Phys. Rev. B 75, 85106 (2007).

[50] R. Glauber. Coherent and Incoherent States of the Radiation Field. Phys. Rev. 131, 2766-2788 (1963).

[51] A. Leggett. Quantum liquids: Bose condensation and Cooper pairing in condensedmatter systems. Oxford University Press, USA (2006). ISBN 019852643.

[52] C. Menotti and N. Trivedi. Spectral weight redistribution in strongly correlated bosons in optical lattices. Phys. Rev. B 77, 235120 (2008). 
[53] S. Trotzky, L. Pollet, F. Gerbier, U. Schnorrberger, I. Bloch, N. V. Prokofev, B. Svistunov, and M. Troyer. Suppression of the critical temperature for superfluidity near the Mott transition. Nature Phys. 6, 998-1004 (2010).

[54] F. Gerbier. Boson Mott Insulators at Finite Temperatures. Phys. Rev. Lett. 99, 120405 (2007).

[55] B. Capogrosso-Sansone, N. Prokof'ev, and B. Svistunov. Phase diagram and thermodynamics of the three-dimensional Bose-Hubbard model. Phys. Rev. B 75, 134302 (2007).

[56] B. Capogrosso-Sansone, S. Söyler, N. Prokof'ev, and B. Svistunov. Monte Carlo study of the two-dimensional Bose-Hubbard model. Phys. Rev. A 77, 015602 (2008).

[57] V. Kashurnikov and B. Svistunov. Exact diagonalization plus renormalization-group theory: Accurate method for a one-dimensional superfluid-insulator-transition study. Phys. Rev. B 53, 11776-11778 (1996).

[58] T. D. Kühner, S. R. White, and H. Monien. One-dimensional Bose-Hubbard model with nearest-neighbor interaction. Phys. Rev. B 61, 12474-12489 (2000).

[59] M. Greiner. Ultracold quantum gases in three-dimensional optical lattice potentials. PhD thesis, Ludwig-Maximilians-Universität München (2003).

[60] D. van Oosten. Quantum gases in optical lattices: the atomic Mott insulator. PhD thesis, Universiteit Utrecht (2004).

[61] S. Fölling. Probing strongly correlated states of ultracold atoms in optical lattices. PhD thesis, Johannes-Gutenberg-Universität Mainz (2008).

[62] S. Will. Interacting bosons and fermions in three-dimensional optical lattice potentials. $\mathrm{PhD}$ thesis, Johannes Gutenberg-Universität Mainz (2011).

[63] A. Altland and B. Simons. Condensed matter field theory. Cambridge University Press, Cambridge (2006). ISBN 0521845084.

[64] M. Haas, U. D. Jentschura, and C. H. Keitel. Comparison of classical and second quantized description of the dynamic Stark shift. Am. J. Phys. 74, 77 (2006).

[65] R. Grimm, M. Weidemüller, and Y. B. Ovchinnikov. Optical dipole traps for neutral atoms. Adv. Atom. Mol. Opt. Phys. 42, 95-170 (2000).

[66] N. W. Ashcroft and N. D. Mermin. Solid State Physics. Saunders, Philadelphia (1976). ISBN 0030839939. 
[67] I. A. S. Milton Abramowitz. Handbook of Mathematical Functions with Formulas, Graphs, and Mathematical Tables. Dover Publications, New York (1972). ISBN 0486612724.

[68] W. Kohn. Analytic Properties of Bloch Waves and Wannier Functions. Phys. Rev. 115, 809-821 (1959).

[69] G. Wannier. The Structure of Electronic Excitation Levels in Insulating Crystals. Phys. Rev. 52, 191-197 (1937).

[70] J. Dalibard. Collisional dynamics of ultra-cold atomic gases. Proceedings of the International School of Physics-Enrico Fermi 321 (1999).

[71] C. J. Pethick and H. Smith. Bose-Einstein condensation in dilute gases. Cambridge University Press, Cambridge (2001). ISBN 0521665809.

[72] E. G. M. van Kempen, S. J. J. M. F. Kokkelmans, D. J. Heinzen, and B. J. Verhaar. Interisotope Determination of Ultracold Rubidium Interactions from Three HighPrecision Experiments. Phys. Rev. Lett. 88, 093201 (2002).

[73] S. Wessel, F. Alet, M. Troyer, and G. Batrouni. Quantum Monte Carlo simulations of confined bosonic atoms in optical lattices. Phys. Rev. A 70, 053615 (2004).

[74] C. Weitenberg. Single-Atom Resolved Imaging and Manipulation in an Atomic Mott Insulator. PhD thesis, Ludwig-Maximilians-Universität München (2011).

[75] D. A. Steck. Rubidium 87 D Line Data. http:/ / steck.us/alkalidata (2010).

[76] V. Bagnato and D. Kleppner. Bose-Einstein condensation in low-dimensional traps. Phys. Rev. A 44, 7439-7441 (1991).

[77] D. S. Petrov, M. Holzmann, and G. V. Shlyapnikov. Bose-Einstein Condensation in Quasi-2D Trapped Gases. Phys. Rev. Lett. 84, 2551-2555 (2000).

[78] A. Görlitz, J. Vogels, A. Leanhardt, C. Raman, T. Gustavson, J. Abo-Shaeer, A. Chikkatur, S. Gupta, S. Inouye, T. Rosenband, and W. Ketterle. Realization of Bose-Einstein Condensates in Lower Dimensions. Phys. Rev. Lett. 87, 130402 (2001).

[79] S. Burger, F. S. Cataliotti, C. Fort, P. Maddaloni, F. Minardi, and M. Inguscio. Quasi-2D Bose-Einstein condensation in an optical lattice. Europhys. Lett. 57, 1-6 (2002).

[80] D. Rychtarik, B. Engeser, H.-C. Nägerl, and R. Grimm. Two-Dimensional BoseEinstein Condensate in an Optical Surface Trap. Phys. Rev. Lett. 92, 173003 (2004).

[81] Z. Hadzibabic, P. Krüger, M. Cheneau, B. Battelier, and J. Dalibard. BerezinskiiKosterlitz-Thouless crossover in a trapped atomic gas. Nature 441, 1118-1121 (2006). 
[82] Z. Hadzibabic, P. Krüger, M. Cheneau, S. P. Rath, and J. Dalibard. The trapped two-dimensional Bose gas: from Bose-Einstein condensation to Berezinskii-KosterlitzThouless physics. New J. Phys. 10, 045006 (2008).

[83] J. Weiner, V. Bagnato, S. Zilio, and P. Julienne. Experiments and theory in cold and ultracold collisions. Rev. Mod. Phys. 71, 1-85 (1999).

[84] F. Gerbier. Boson Mott insulators at finite temperatures. Phys. Rev. Lett. 99, 120405 (2007).

[85] P. Ma, L. Pollet, and M. Troyer. Measuring the equation of state of trapped ultracold bosonic systems in an optical lattice with in situ density imaging. Phys. Rev. A 82, 033627 (2010).

[86] F. Gerbier, A. Widera, S. Fölling, O. Mandel, T. Gericke, and I. Bloch. Phase Coherence of an Atomic Mott Insulator. Phys. Rev. Lett. 95, 050404 (2005).

[87] A. B. Kuklov and B. V. Svistunov. Counterflow Superfluidity of Two-Species Ultracold Atoms in a Commensurate Optical Lattice. Phys. Rev. Lett. 90, 100401 (2003).

[88] L.-M. Duan, E. Demler, and M. Lukin. Controlling Spin Exchange Interactions of Ultracold Atoms in Optical Lattices. Phys. Rev. Lett. 91, 090402 (2003).

[89] S. Trotzky, P. Cheinet, S. Fölling, M. Feld, U. Schnorrberger, A. M. Rey, A. Polkovnikov, E. A. Demler, M. D. Lukin, and I. Bloch. Time-resolved observation and control of superexchange interactions with ultracold atoms in optical lattices. Science 319, 295 (2008).

[90] S. Nascimbène, Y.-A. Chen, M. Atala, M. Aidelsburger, S. Trotzky, B. Paredes, and I. Bloch. Experimental Realization of Plaquette Resonating Valence-Bond States with Ultracold Atoms in Optical Superlattices. Phys. Rev. Lett. 108, 205301 (2012).

[91] E. Kapit and E. Mueller. Even-odd correlation functions on an optical lattice. Phys. Rev. A 82, 013644 (2010).

[92] F. Verstraete, M. Martín-Delgado, and J. Cirac. Diverging Entanglement Length in Gapped Quantum Spin Systems. Phys. Rev. Lett. 92, 087201 (2004).

[93] M. Zwolak and G. Vidal. Mixed-State Dynamics in One-Dimensional Quantum Lattice Systems: A Time-Dependent Superoperator Renormalization Algorithm. Phys. Rev. Lett. 93, 207205 (2004).

[94] E. Toth and P. Blakie. Thermally induced coherence in a Mott insulator of bosonic atoms. Phys. Rev. A 83, 021601(R) (2011).

[95] F. Anfuso and A. Rosch. Fragility of string orders. Phys. Rev. B 76, 085124 (2007). 
[96] M. den Nijs and K. Rommelse. Preroughening transistions in crystal surfaces and valence-bond phases in quantum spin chains. Phys. Rev. B 40, 4709 (1989).

[97] H. Kruis, I. McCulloch, Z. Nussinov, and J. Zaanen. Geometry and the hidden order of Luttinger liquids: The universality of squeezed space. Phys. Rev. B 70, 075109 (2004).

[98] D. Pérez-García, M. Wolf, M. Sanz, F. Verstraete, and J. I. Cirac. String Order and Symmetries in Quantum Spin Lattices. Phys. Rev. Lett. 100, 167202 (2008).

[99] E. G. Dalla Torre, E. Berg, and E. Altman. Hidden Order in 1D Bose Insulators. Phys. Rev. Lett. 97, 260401 (2006).

[100] E. Berg, E. Dalla Torre, T. Giamarchi, and E. Altman. Rise and fall of hidden string order of lattice bosons. Phys. Rev. B 77, 245119 (2008).

[101] E. Kim, G. Fáth, J. Sólyom, and D. Scalapino. Phase transitions between topologically distinct gapped phases in isotropic spin ladders. Phys. Rev. B 62, 14965-14974 (2000).

[102] E. Kim and J. Sólyom. Opening of the Haldane gap in anisotropic two- and four-leg spin ladders. Phys. Rev. B 60, 15230-15244 (1999).

[103] F. Anfuso and A. Rosch. String order and adiabatic continuity of Haldane chains and band insulators. Phys. Rev. B 75, 144420 (2007).

[104] A. Montorsi and M. Roncaglia. Non-local order parameters for the 1D Hubbard model. arXiv:1207.3426 (2012).

[105] F. Verstraete, M. Popp, and J. Cirac. Entanglement versus Correlations in Spin Systems. Phys. Rev. Lett. 92, 027901 (2004).

[106] M. Popp, F. Verstraete, M. Martín-Delgado, and J. Cirac. Localizable entanglement. Phys. Rev. A 71, 042306 (2005).

[107] L. Venuti and M. Roncaglia. Analytic Relations between Localizable Entanglement and String Correlations in Spin Systems. Phys. Rev. Lett. 94, 207207 (2005).

[108] J. García-Ripoll, M. Martin-Delgado, and J. Cirac. Implementation of Spin Hamiltonians in Optical Lattices. Phys. Rev. Lett. 93, 250405 (2004).

[109] F. D. M. Haldane. 'Luttinger liquid theory' of one-dimensional quantum fluids. I. Properties of the Luttinger model and their extension to the general 1D interacting spinless Fermi gas. J. Phys. C 14, 2585-2609 (1981).

[110] F. D. M. Haldane. Effective Harmonic-Fluid Approach to Low-Energy Properties of One-Dimensional Quantum Fluids. Phys. Rev. Lett. 47, 1840-1843 (1981). 
[111] T. Giamarchi. Quantum Physics in One Dimension. Oxford University Press (2003). ISBN 9780198525004.

[112] T. Giamarchi. Strong Correlations in Low Dimensional Systems. AIP Conference Proceedings 846, 94-129 (2006).

[113] M. Cazalilla, R. Citro, T. Giamarchi, E. Orignac, and M. Rigol. One dimensional bosons: From condensed matter systems to ultracold gases. Rev. Mod. Phys. 83, 1405-1466 (2011).

[114] H. Büchler, G. Blatter, and W. Zwerger. Commensurate-Incommensurate Transition of Cold Atoms in an Optical Lattice. Phys. Rev. Lett. 90, 130401 (2003).

[115] S. P. Rath, W. Simeth, M. Endres, and W. Zwerger. Non-local order in Mott insulators, Duality and Wilson Loops. arXiv:1302.0693 (2013).

[116] T. Kühner and H. Monien. Phases of the one-dimensional Bose-Hubbard model. Phys. Rev. B 58, R14741-R14744 (1998).

[117] R. Kubo. Generalized Cumulant Expansion Method. J. Phys. Soc. Jpn. 17, 11001120 (1962).

[118] H. Kramers and G. Wannier. Statistics of the Two-Dimensional Ferromagnet. Part I. Phys. Rev. 60, 252-262 (1941).

[119] E. Fradkin and L. Susskind. Order and disorder in gauge systems and magnets. Phys. Rev. D 17, 2637-2658 (1978).

[120] M. E. Peskin. Mandelstam-'t Hooft duality in abelian lattice models. Ann. Phys. 113, 122-152 (1978).

[121] R. Savit. Duality in field theory and statistical systems. Rev. Mod. Phys. 52, 453-487 (1980).

[122] J. B. Kogut. An introduction to lattice gauge theory and spin systems. Rev. Mod. Phys. 51, 659-713 (1979).

[123] M. Fisher. Duality in low dimensional quantum field theories. In Strong interactions in low dimensions, pages 419-438. Springer (2004). ISBN 9781402017988.

[124] L. Balents, L. Bartosch, A. Burkov, S. Sachdev, and K. Sengupta. Competing Orders and Non-Landau-Ginzburg-Wilson Criticality in (Bose) Mott Transitions. Prog. Theor. Phys. Supplement 160, 314-336 (2005).

[125] M. Fisher and D. Lee. Correspondence between two-dimensional bosons and a bulk superconductor in a magnetic field. Phys. Rev. B 39, 2756-2759 (1989). 


\section{Bibliography}

[126] W. Zwerger. Global and Local Phase Coherence in Dissipative Josephson-Junction Arrays. Europhys. Lett. 9, 421-426 (1989).

[127] I. Herbut. Dual theory of the superfluid-Bose-glass transition in the disordered BoseHubbard model in one and two dimensions. Phys. Rev. B 57, 13729-13742 (1998).

[128] P. Pfeuty. The one-dimensional Ising model with a transverse field. Ann. Phys. 57, 79-90 (1970).

[129] F. J. Wegner. Duality in Generalized Ising Models and Phase Transitions without Local Order Parameters. J. Math. Phys. 12, 2259 (1971).

[130] K. Wilson. Confinement of quarks. Phys. Rev. D 10, 2445-2459 (1974).

[131] H. F. Song, S. Rachel, and K. Le Hur. General relation between entanglement and fluctuations in one dimension. Phys. Rev. B 82, 012405 (2010).

[132] H. F. Song, C. Flindt, S. Rachel, I. Klich, and K. Le Hur. Entanglement entropy from charge statistics: Exact relations for noninteracting many-body systems. Phys. Rev. B 83, 161408 (2011).

[133] H. Song, S. Rachel, C. Flindt, I. Klich, N. Laflorencie, and K. Le Hur. Bipartite fluctuations as a probe of many-body entanglement. Phys. Rev. B 85, 035409 (2012).

[134] S. Rachel, N. Laflorencie, H. F. Song, and K. Le Hur. Detecting Quantum Critical Points using Bipartite Fluctuations. Phys. Rev. Lett. 108, 116401 (2012).

[135] G. C. Levine, M. J. Bantegui, and J. A. Burg. Full counting statistics in a disordered free fermion system. arXiv:1201.3933 (2012).

[136] M. Kardar. Statistical physics of fields. Cambridge University Press, Cambridge (2007). ISBN 052187341X.

[137] S. Weinberg. The quantum theory of fields, Vol. 2. Cambridge University Press, Cambridge (1996). ISBN 0521670543.

[138] E. Altman and A. Auerbach. Oscillating Superfluidity of Bosons in Optical Lattices. Phys. Rev. Lett. 89, 250404 (2002).

[139] R. Sooryakumar and M. Klein. Raman Scattering by Superconducting-Gap Excitations and Their Coupling to Charge-Density Waves. Phys. Rev. Lett. 45, 660-662 (1980).

[140] P. Littlewood and C. Varma. Gauge-Invariant Theory of the Dynamical Interaction of Charge Density Waves and Superconductivity. Phys. Rev. Lett. 47, 811-814 (1981). 
[141] C. Rüegg, B. Normand, M. Matsumoto, A. Furrer, D. McMorrow, K. Krämer, H. Güdel, S. Gvasaliya, H. Mutka, and M. Boehm. Quantum Magnets under Pressure: Controlling Elementary Excitations in TlCuCl3. Phys. Rev. Lett. 100, 205701 (2008).

[142] A. Polkovnikov, E. Altman, E. Demler, B. Halperin, and M. Lukin. Decay of superfluid currents in a moving system of strongly interacting bosons. Phys. Rev. A 71, 063613 (2005).

[143] A. Polkovnikov, S. Sachdev, and S. Girvin. Nonequilibrium Gross-Pitaevskii dynamics of boson lattice models. Phys. Rev. A 66, 53607 (2002).

[144] K. Sengupta and N. Dupuis. Mott-insulator-to-superfluid transition in the BoseHubbard model: A strong-coupling approach. Phys. Rev. A 71, 033629 (2005).

[145] S. Huber, B. Theiler, E. Altman, and G. Blatter. Amplitude Mode in the Quantum Phase Model. Phys. Rev. Lett. 100, 050404 (2008).

[146] T. D. Graß, F. E. A. Santos, and A. Pelster. Real-time Ginzburg-Landau theory for bosons in optical lattices. Laser Phys. 21, 1459-1463 (2011).

[147] U. Bissbort, S. Götze, Y. Li, J. Heinze, J. S. Krauser, M. Weinberg, C. Becker, K. Sengstock, and W. Hofstetter. Detecting the Amplitude Mode of Strongly Interacting Lattice Bosons by Bragg Scattering. Phys. Rev. Lett. 106, 205303 (2011).

[148] J. Schachenmayer, G. Pupillo, and A. J. Daley. Time-dependent currents of onedimensional bosons in an optical lattice. New J. Phys. 12, 025014 (2010).

[149] Y. Nambu. Quasi-Particles and Gauge Invariance in the Theory of Superconductivity. Phys. Rev. 117, 648-663 (1960).

[150] J. Goldstone. Field theories with Superconductor solutions. Il Nuovo Cimento 19, 154-164 (1961).

[151] P. Higgs. Broken Symmetries and the Masses of Gauge Bosons. Phys. Rev. Lett. 13, 508-509 (1964).

[152] P. M. Chaikin and T. C. Lubensky. Principles of condensed matter physics. Cambridge University PressCambridge (1995).

[153] C. Varma. Higgs Boson in Superconductors. J. Low Temp. Phys. 126, 901-909 (2002).

[154] M. A. Cazalilla, A. F. Ho, and T. Giamarchi. Interacting Bose gases in quasi-onedimensional optical lattices. New J. Phys. 8, 158-158 (2006). 


\section{Bibliography}

[155] K. W. Mahmud, E. N. Duchon, Y. Kato, N. Kawashima, R. T. Scalettar, and N. Trivedi. Finite-temperature study of bosons in a two-dimensional optical lattice. Phys. Rev. B 84, 054302 (2011).

[156] L. Pollet and N. Prokof'ev. Personal communication.

[157] M. Endres, T. Fukuhara, D. Pekker, M. Cheneau, P. Schauss, C. Gross, E. Demler, S. Kuhr, and I. Bloch. The 'Higgs' amplitude mode at the two-dimensional superfluid/Mott insulator transition. Nature 487, 454-458 (2012).

[158] A. Polkovnikov, E. Altman, and E. Demler. Interference between independent fluctuating condensates. Proc. Natl. Acad. Sci. 103, 6125-6129 (2006).

[159] S. Hofferberth, I. Lesanovsky, T. Schumm, A. Imambekov, V. Gritsev, E. Demler, and J. Schmiedmayer. Probing quantum and thermal noise in an interacting manybody system. Nature Phys. 4, 489-495 (2008).

[160] J. Honer, H. Weimer, T. Pfau, and H. Büchler. Collective Many-Body Interaction in Rydberg Dressed Atoms. Phys. Rev. Lett. 105, 160404 (2010).

[161] G. Pupillo, A. Micheli, M. Boninsegni, I. Lesanovsky, and P. Zoller. Strongly Correlated Gases of Rydberg-Dressed Atoms: Quantum and Classical Dynamics. Phys. Rev. Lett. 104, 223002 (2010).

[162] F. Cinti, P. Jain, M. Boninsegni, A. Micheli, P. Zoller, and G. Pupillo. Supersolid Droplet Crystal in a Dipole-Blockaded Gas. Phys. Rev. Lett. 105, 135301 (2010).

[163] N. Henkel, R. Nath, and T. Pohl. Three-Dimensional Roton Excitations and Supersolid Formation in Rydberg-Excited Bose-Einstein Condensates. Phys. Rev. Lett. 104, 195302 (2010).

[164] N. Henkel, F. Cinti, P. Jain, G. Pupillo, and T. Pohl. Supersolid Vortex Crystals in Rydberg-Dressed Bose-Einstein Condensates. Phys. Rev. Lett. 108, 265301 (2012).

[165] M. Ganahl, E. Rabel, F. Essler, and H. Evertz. Observation of Complex Bound States in the Spin-1/2 Heisenberg XXZ Chain Using Local Quantum Quenches. Phys. Rev. Lett. 108, 077206 (2012).

[166] A. Recati, P. O. Fedichev, W. Zwerger, and P. Zoller. Spin-charge separation in ultracold quantum gases. Phys. Rev. Lett. 90, 020401 (2003).

[167] C. Kollath, U. Schollwöck, and W. Zwerger. Spin-Charge Separation in Cold Fermi Gases: A Real Time Analysis. Phys. Rev. Lett. 95, 176401 (2005).

[168] A. Kleine, C. Kollath, I. McCulloch, T. Giamarchi, and U. Schollwöck. Spincharge separation in two-component Bose gases. Phys. Rev. A 77, 013607 (2008). 
[169] K. Henderson, C. Ryu, C. MacCormick, and M. G. Boshier. Experimental demonstration of painting arbitrary and dynamic potentials for Bose-Einstein condensates. New J. Phys. 11, 043030 (2009).

[170] B. Zimmermann, T. Müller, J. Meineke, T. Esslinger, and H. Moritz. Highresolution imaging of ultracold fermions in microscopically tailored optical potentials. New J. Phys. 13, 043007 (2011).

[171] J.-P. Brantut, J. Meineke, D. Stadler, S. Krinner, and T. Esslinger. Conduction of ultracold fermions through a mesoscopic channel. Science 337, 1069-1071 (2012).

[172] A. Ramanathan, K. Wright, S. Muniz, M. Zelan, W. Hill, C. Lobb, K. Helmerson, W. Phillips, and G. Campbell. Superflow in a Toroidal Bose-Einstein Condensate: An Atom Circuit with a Tunable Weak Link. Phys. Rev. Lett. 106, 130401 (2011).

[173] S. Rath, B. Spivak, and W. Zwerger. Quantum Capillary Waves at the SuperfluiMott-Insulator Interface. Phys. Rev. Lett. 107, 155703 (2011).

[174] D. R. Fernandes, F. Sievers, N. Kretzschmar, S. Wu, C. Salomon, and F. Chevy. Sub-Doppler laser cooling of fermionic $40 \mathrm{~K}$ atoms in three-dimensional gray optical molasses. arXiv:1210.1310 (2012).

[175] T. Wilk, A. Gaetan, C. Evellin, J. Wolters, Y. Miroshnychenko, P. Grangier, and A. Browaeys. Entanglement of two individual neutral atoms using Rydberg blockade. Phys. Rev. Lett. 104, 10502 (2010).

[176] L. Isenhower, E. Urban, X. L. Zhang, A. T. Gill, T. Henage, T. A. Johnson, T. G. Walker, and M. Saffman. Demonstration of a neutral atom controlled-NOT quantum gate. Phys. Rev. Lett. 104, 010503 (2010).

[177] C. Weitenberg, S. Kuhr, K. Mø lmer, and J. Sherson. Quantum computation architecture using optical tweezers. Phys. Rev. A 84, 032322 (2011).

[178] R. Raussendorf and H. J. Briegel. A one-way quantum computer. Phys. Rev. Lett. 86, 5188-5191 (2001).

[179] H. J. Briegel, D. E. Browne, W. Dür, R. Raussendorf, and M. Van Den Nest. Measurement-based quantum computation. Nature Phys. 5, 19-26 (2009).

[180] D. Jaksch, H.-J. Briegel, J. I. Cirac, C. W. Gardiner, and P. Zoller. Entanglement of atoms via cold controlled collisions. Phys. Rev. Lett. 82, 1975-1978 (1999).

[181] O. Mandel, M. Greiner, A. Widera, T. Rom, T. Hänsch, and I. Bloch. Controlled collisions for multiparticle entanglement of optically trapped atoms. Nature 425, 937940 (2003). 
[182] M. Garwood and L. DelaBarre. The return of the frequency sweep: Designing adiabatic pulses for contemporary NMR. J. Magn. Reson. 153, 155-177 (2001).

[183] O. Loesdau. Aufbau und Charakterisierung eines Doppel-MOT-Systems. Diploma thesis, Johannes-Gutenberg-Universität Mainz (2008).

[184] K. Dieckmann, R. Spreeuw, M. Weidemüller, and J. Walraven. Two-dimensional magneto-optical trap as a source of slow atoms. Phys. Rev. A 58, 3891-3895 (1998).

[185] J. Petersen. Optischer Transport ultrakalter Atome. Diploma thesis, JohannesGutenberg-Universität Mainz (2009).

[186] D. Stamper-Kurn, H.-J. Miesner, A. Chikkatur, S. Inouye, J. Stenger, and W. Ketterle. Reversible Formation of a Bose-Einstein Condensate. Phys. Rev. Lett. 81, 21942197 (1998).

[187] U. Schollwöck. The density-matrix renormalization group. Rev. Mod. Phys. 77, 259-315 (2005).

[188] T. Kennedy and H. Tasaki. Hidden Z2xZ2 symmetry breaking in Haldane-gap antiferromagnets. Phys. Rev. B 45, 304-307 (1992).

[189] H. Ueda, H. Nakano, and K. Kusakabe. Finite-size scaling of string order parameters characterizing the Haldane phase. Phys. Rev. B 78, 224402 (2008).

[190] M. Dalmonte, M. Di Dio, L. Barbiero, and F. Ortolani. Homogeneous and inhomogeneous magnetic phases of constrained dipolar bosons. Phys. Rev. B 83, 155110 (2011).

[191] N. V. Prokof'ev, B. V. Svistunov, and I. S. Tupitsyn. Exact, complete, and universal continuous-time worldline Monte Carlo approach to the statistics of discrete quantum systems. JETP 87, 310-321 (1998).

[192] L. Pollet, K. Houcke, and S. Rombouts. Engineering local optimality in quantum Monte Carlo algorithms. J. Comp. Phys. 225, 2249-2266 (2007).

[193] L. Pollet, C. Kollath, K. Van Houcke, and M. Troyer. Temperature changes when adiabatically ramping up an optical lattice. New J. Phys. 10, 065001 (2008).

[194] C. Cohen-Tannoudji, J. Dupont-Roc, and G. Grynberg. Photons and Atoms: Introduction to Quantum Electrodynamics. Wiley-Interscience (1989). ISBN 0471845264. 


\section{Danksagung}

Mein Dank gebührt vielen Menschen ohne die diese Arbeit nicht möglich gewesen wäre:

Zunächst möchte ich Prof. Immanuel Bloch danken, dass ich die Arbeit in seiner Gruppe unter besten Bedingungen durchführen konnte. Dies betrifft nicht nur die Betreuung selbst sondern auch das Vorhandensein einer offenen und konstruktiven Atmosphäre sowie hervorragender Arbeitsbedingungen im Allgemeinen. Ich konnte sehr viel von ihm lernen, nicht nur über Physik, sondern auch über die richtige Präsentation von Ergebnissen, sei es in Vorträgen oder in Publikationen. Besonders schätze ich seine Offenheit für neue Ideen, seinen "Drive", und dass er sich trotz vollem Terminkalender immer Zeit für Hilfestellung aller Art und Diskussionen nimmt. Ich bin auch besonders dankbar dafür, dass ich an verschiedenen Konferenzen und Schulen teilnehmen konnte; insbesondere mein Aufenthalt in Israel und die Schule in Les Houches waren prägende Erlebnisse, die sehr wichtig für das Gelingen dieser Arbeit waren.

Nicht minder dankbar bin ich Prof. Stefan Kuhr, unter dessen Anleitung das Single Atoms Team das Experiment zum Erfolg führen konnte. Es war eine Freude mit ihm zu arbeiten und von ihm zu lernen; über Physik, das richtige Schreiben und Präsentieren, aber auch über all die technischen Dinge die man im Labor wissen muss und für die es keinen besseren Lehrer gibt. Ich möchte mich bedanken dafür, dass er immer da war wenn es mal brenzlig wurde, immer ein offenes Ohr hatte für neue Ideen und für Anliegen aller Art, aber auch klare Kritik übte wenn man mal auf dem falschen Weg war, nicht nur was die Physik angeht. Seine Einladungen und Feiern nach erfolgreicher Publikation sind sowieso Legende.

Ich möchte mich generell bei allen ehemaligen und jetzigen Mitgliedern des Single Atoms Teams bedanken. Unsere Ergebnisse sind vor Allem das Resultat einer sehr guten Teamarbeit und weniger die Leistung eines Einzelnen. Insbesondere danke ich:

Jakob Sherson, für die Zusammenarbeit und Hilfe in den ersten Jahren und die vielen Dinge im Labor und außerhalb, die ich von ihm lernen konnte. Christof Weitenberg, meinem PhD Kollegen, mit dem es sehr viel Spass gemacht hat zu arbeiten. Für die vielen Diskussionen über Physik, Gott und die Welt und seinen schrägen Humor. Nicht dankbar bin ich dafür, dass Süßigkeiten oft nur beschränkte Haltbarkeit hatten, wenn er im Labor war. Takeshi Fukuhara, meinem Bürokollegen 
und Messpartner für das particle-hole und Higgs Projekt. Ohne seine selbstlose Hilfe wären beide Projekte nicht möglich gewesen. Marc Cheneau, für die super Zusammenarbeit, insbesondere um das Experiment stabil zu bekommen, und für die vielen Diskussionen über Physik. Peter Schauß, für die Hilfe in den letzten beiden Jahren, insbesondere was Programming angeht und das Korrekturlesen der gesamten Arbeit. Christian Groß, für die vielen guten Diskussionen und seine Hilfe im letzten Jahr. Sebastian Hild und Johannes Zeiher, für die gute Zusammenarbeit und fürs Korrekturlesen. Meinen ehemaligen Kollegen Olli Lösdau und Jan Petersen, für den Spass und die gute Zusammenarbeit.

Ich möchte mich generell bei unseren Theorie-Kollaboratoren bedanken. Ohne die gute Zusammenarbeit wären sowohl das particle-hole als auch das Higgs Projekt kaum möglich gewesen. Dies gilt insbesondere für:

Leonardo Mazza, ohne dessen selbstlose Hilfe, gerade in der Anfangsphase, das particle-hole Paper nicht zustande gekommen wäre. David Pekker, für die gute und sehr intensive Zusammenarbeit beim Higgs paper, sowie für zahlreiche Skype-Gespräche zu unmenschlichen Uhrzeiten. Lode Pollet für die sehr gute Zusammenarbeit beim particle-hole paper und die Diskussionen über Higgs. Mari Carmen Bañuls für die reibungslose Zusammenarbeit beim particle-hole paper. Eugene Demler für die Hilfe und Diskussionen beim Higgs paper. Wilhelm Zwerger für die Diskussionen über Higgs und string order und seinen generellen Rat. Steffen Rath und Wolgang Simeth für die Diskussionen über string order in $1 \mathrm{~d}$ und vor allem 2d.

Desweiteren möchte ich mich bei Emanuele Dalla Torre und Ehud Altman für den initialen Impuls zur string order Messung und für die darauf folgenden Diskussionen bedanken. Für wichtige Diskussionen über Higgs Moden danke ich auch Subir Sachdev, Nikolay Prokov'ev und Daniel Podolsky. Sowie Ignacio Cirac, Christophe Salomon, und Nigel Cooper für wertvolle Diskussion über string order und Higgs.

Ich möchte mich auch bei allen nichtwissenschaftlichen Mitarbeitern der Bloch Gruppe bedanken für die Hilfe in den letzten Jahren. Insbesondere bei unseren jetzigen und ehemaligen Sekretärinnen Marianna Kargl, Ildiko Kecskesi, Zohra Hauck und Christine Best für die Unterstützung. Sowie bei allen unseren jetzigen und ehemaligen Technikern, insbesondere Karsten Förster, Markus Böhm, Michael Pruscha und Heinz Lenk ohne deren Hilfe die technische Umsetzung des Experiments nicht möglich gewesen wäre. Zudem gilt mein Dank Allen die das Max-Planck Institut für Quantenoptik am laufen halten und damit die Voraussetzungen für unsere Experimente schaffen.

Zudem gilt mein Dank allen Kollegen aus der Bloch Gruppe für Diskussionen, Rat und Hilfe bei Aufbau des Experiments sowie dem Vielen Spaß nebenbei. Insbesondere Sebastian Will, Uli Scheider, Stefan Trotzky und Simon Braun für alles Mögliche, vor allem für Geselligkeit und Gespräche über Physik, Musik, Burger, 
Fußball, Eichhörnchen und was einen sonst noch glücklich macht.

Ich möchte mich bei meinen besten Freunden Hausi, Knödel und Anne für ihre Unterstützung bedanken.

Besonderer Dank gebührt meinen Eltern Luitgard und Eugen, sowie meinem Bruder Gerald für ihre bedingungslose Hilfe in allen Lebenslagen.

Last but not Least, möchte ich mich bei Tracy bedanken für so viele Dinge für die hier nicht genug Platz ist, besonders aber für die Unterstützung gerade in der heißen Phase, in der ich sicher nicht leicht zu ertragen war, sowie für die Korrektur meines german English. 